\title{
Entwicklung von Lanthanoid-Tags für die biomolekulare NMR-Spektroskopie
}

\author{
Dissertation \\ zur Erlangung des mathematisch-naturwissenschaftlichen Doktorgrades \\ Doctor rerum naturalium \\ der Georg-August-Universität Göttingen
}

vorgelegt von

Fabian Peters

aus Oldenburg

Göttingen 2010 
D 7

Referent:

Prof. Dr. Ulf Diederichsen,

Georg-August-Universität, Göttingen

Korreferent:

Prof. Dr. Christian Griesinger,

Max-Planck-Institut für biophysikalische Chemie, Göttingen

Tag der mündlichen Prüfung: 15. Dezember 2010 
für Oma Lülala 



\section{Zusammenfassung}

Paramagnetische Markierungen, so genannte Tags, finden in der Protein-NMR-Spektroskopie immer breitere Anwendung. Ein chelatisierender Ligand wird dabei mit einem paramagnetischen Lanthanoidion beladen und über eine Disulfidbrücke selektiv an einzelnen Cystein-Aminosäureresten des Proteins verankert. Wird eine derart markierte NMR-Probe in ein starkes Magnetfeld eingebracht, so erfolgt eine partielle Ausrichtung der Gesamtheit der Moleküle entlang des Feldes, bedingt durch die magnetisch anisotrope Suszeptibilität der paramagnetischen Ionen. Durch die kovalente Verbindung mit dem Liganden wird die Vorzugsorientierung des Ions auf das zu untersuchende Protein übertragen. Residuale dipolare Kopplungen (RDCs), die in isotroper Lösung durch Diffusion und Gleichverteilung zu Null gemittelt sind, sowie Pseudokontaktverschiebungen (PCSs), werden messbar. Für die dreidimensionale Strukturaufklärung sind beide Messgrößen sehr wertvolle Parameter, da sie neben Distanz- vor allem Winkelinformationen in Form relativer Orientierungen von Interspinvektoren enthalten. Eine besondere Bedeutung kommt der paramagnetischen NMR-Spektroskopie bei der Beschreibung relativer Bewegungen zweier Proteindomänen zu, da RDCs dynamische Prozesse im Bereich von Piko- bis Millisekunden beschreiben.

Bislang wurden im Arbeitskreis EDTA-basierte Tags verwendet. Hiermit traten jedoch zahlreiche Probleme auf, sowohl in Bezug auf die Probenherstellung als auch die Größe der RDCs und deren Korrelation mit den berechneten Daten. Innerhalb der vorliegenden Arbeit wurde ein neuartiger, paramagnetischer Tag auf der Grund-<smiles>CS(=O)(=O)SC[C@@H](NC(=O)c1ccc(C(CN(CC(=O)O)CC(=O)O)(CN(CC(=O)O)CC(=O)O)CN(CC(=O)O)CC(=O)O)cc1)C(=O)O</smiles>

Abb. 1: Cys-Ph-TAHA. 
lage des neunzähnigen TAHA-Liganden (triaminohexaacetate) entwickelt, da dieser Chelator die Koordinationssphäre von Lanthanoidionen besser absättigt als EDTA. Die Erhöhung der Koordinationszahl unterbindet ungewollte Interaktionen des Proteins mit dem Metallion. Gleichzeitig lässt sich erwarten, dass ein stärkerer sterischer Anspruch des Tags zu einer Verstärkung der Molekülausrichtung führt.

Der Cys-Ph-TAHA-Tag (Cysteinyl-Phenyl-TAHA, siehe Abbildung 1) wurde in neun Stufen erfolgreich dargestellt. Dabei ließ die Schlichtheit der Synthese auch Maßstäbe von mehreren hundert Milligramm zu, wodurch Cys-Ph-TAHA sehr leicht zugänglich ist. Für die Herstellung Tag-modifizierter, beladener Proteinproben wurde eine effektive und reproduzierbare Methode entwickelt, mit der der zuvor beladene Tag am Protein angebracht werden kann. Auch dieses Protokoll zeichnet sich durch eine einfache Durchführbarkeit aus.

Der mit paramagnetischem $\mathrm{Tb}^{3+}$ und $\mathrm{Tm}^{3+}$ vorbeladene Cys-Ph-TAHA-Tag wurde an zwei verschiedene Ubiquitin-Cysteinmutanten (T12C und S57C) gebunden. Ubiquitin eignet sich hervorragend als Testprotein, da es einerseits relativ unempfindlich gegenüber diversen äußeren Einflüssen, wie pH Wert, Temperatur oder Puffersystem ist und andererseits die Aufnahme von gut aufgelösten NMR-Spektren ermöglicht. Darüber hinaus ist die NMR-Struktur von Ubiquitin bekannt und konnte als Referenz bei der Zurückrechnung der RDCs und PCSs genutzt werden.

Aus den Spektren konnten große RDCs und PCSs von hoher Qualität extrahiert werden, die in exzellenter Übereinstimmung mit den zurück berechneten Werten waren. Es zeigte sich, dass der neunzähnige TAHA-Ligand für die Anforderungen der paramagnetischen NMR-Spektroskopie sehr gut geeignet ist.

Mit dem Cys-Ph-TAHA-Tag wurde ein viel versprechendes Werkzeug für die NMRSpektroskopie entwickelt. Zukünftig soll Cys-Ph-TAHA zur Strukturaufklärung und -validierung eingesetzt werden. Darüber hinaus ermöglicht die Größe der erhaltenen RDCs dynamische Untersuchungen an Proteinen mit mehreren Domänen, wie beispielsweise Calmodulin. 


\section{Summary}

Paramagnetic labels (tags) have gained increasing interest and application in protein NMR spectroscopy. A chelating ligand is loaded with paramagnetic lanthanide ions and selectively linked via a disulfide bridge to a single cysteine residue. If such a labeled protein sample is introduced into a strong magnetic field, a partial alignment of the molecules occurs along the magnetic field due the anisotropic magnetic susceptibility of the paramagnetic ions. In consequence of the covalent connection with the ligand, the preferred orientation of the ion is transferred to the protein. Residual dipolar couplings (RDCs), which are averaged to zero in isotropic solution, become observable as well as pseudocontact shifts (PCSs). For the determination of threedimensional structures both measurands are valuable parameters since they contain information about distances and relative orientations of inter spin vectors. Paramagnetic NMR spectroscopy is of special importance when it comes to the description of relative protein domain motions since RDCs can report on dynamic processes from pico- to milliseconds. To date in our group, tags based on EDTA were used. But with these tags problems had occurred regarding the sample preparation as well as the size of the induced RDCs and their correlation with the back-calculated data. Within this dissertation a new paramagnetic tag based on the nonadentate TAHA ligand (triaminohexaacetate) was developed. The TAHA ligand provides a higher saturation of the coordination sphere of lanthanide ions than EDTA. This increase of coordination should suppress unwanted interactions of the protein side chains<smiles>CS(=O)(=O)SC[C@H](NC(=O)c1ccc(C(CN(CC(=O)O)CC(=O)O)(CN(CC(=O)O)CC(=O)O)CN(CC(=O)O)C(=O)O)cc1)C(=O)O</smiles>

Abb. 2: Cys-Ph-TAHA. 
viii

with the metal ion. At the same time one can then expect that the sterically higher demanding ligand results in an enhanced molecular alignment.

The Cys-Ph-TAHA tag (cysteinyl-phenyl-TAHA, figure 2) was successfully synthesized in nine steps. The simplicity of the synthesis allowed for the large scale production several hundred milligrams making Cys-Ph-TAHA easily accessible. Additionally, an effective and reproducible method for the preparation of tag-modified and loaded protein samples was established. This procedure is again characterized by its easiness.

Cys-Ph-TAHA, pre-loaded with either paramagnetic $\mathrm{Tb}^{3+}$ or $\mathrm{Tm}^{3+}$, was tagged to two different single cysteine mutants of ubiquitin (T12C and S57C). Ubiquitin is excellently suitable as a test-protein because it is on the one hand very stable against various conditions like $\mathrm{pH}$, temperature, or the buffer system and produces well resolved NMR spectra. Furthermore, the liquid-state NMR structure of ubiquitin is well known and was used as a reference for the back-calculation of the RDCs and PCSs.

Large RDCs and PCSs of high quality were extracted from the spectra. The experimental data were in excellent agreement with the back-calculated values. It was shown, that the TAHA ligand satisfies the demands of paramagnetic NMR spectroscopy.

With Cys-Ph-TAHA a promising tool for liquid state NMR spectroscopy was developed. In the future, Cys-Ph-TAHA shall be used both for structure determination and validation. Additionally, the sizes of the obtained RDCs allow further investigations of protein dynamics. 


\section{Zugehörige Publikationen}

Peters, F., Maestre Martinez, M., Leonov, A., Kovačič, L., Becker, S. Boelens, R., \& C. Griesinger, Cys-Ph-TAHA: A lanthanide binding tag for RDC and PCS enhanced protein NMR, Manuskript in Vorbereitung. 


\section{Danksagungen}

Mein besonderer Dank gilt meinem Doktorvater, Herrn Prof. Dr. Griesinger, für die interessante Aufgabenstellung, die gewährten Freiheiten zur Bearbeitung des Themas und seine stete Diskussionsbereitschaft.

Herrn Prof. Dr. Diederichsen möchte ich für die Betreuung dieser externen Doktorarbeit von Seiten der Universität Göttingen danken. Ohne seine bereitwillige und freundliche Unterstützung wäre diese Arbeit nicht möglich gewesen.

Dr. Andrei Leonov danke ich für die Unterstützung beim synthetischen Teil dieser Arbeit, Dr. Stefan Becker und Karin Giller danke ich für die Herstellung des Proteins.

Dr. Mitcheell Maestre Martinez bin ich sehr dankbar für die enge und fruchtbare Zusammenarbeit im letzten Jahr dieser Arbeit. Dr. Holger Schmidt danke ich für die Einweisungen in die Protein-NMR-Spektroskopie.

Kerstin Overkamp und Gerhard Wolf danke ich für die Aufnahme von Massenspektren und HPLC-Chromatogrammen, sowie die Einarbeitungen in die HPLC-Anlage und das Massenspektrometer. Danke auch an Dr. Dirk Bockelmann und Jürgen Arve für die Hilfe bei Computer-Problemen aller Art.

Ein besonderer Dank gilt Dr. Edith Wöltjen, Asst. Prof. Dr. Mate Erdélyi, Dr. Volker Klaukien, Florian Siepel und Sebastian Täubert für die tolle Atmosphäre im Labor. Gleiches gilt für die Bürokollegen Dr. Luigi Russo, Philip Lottmann und Prof. Dr. Uwe Reinscheid.

Des weiteren möchte ich Dr. Mitcheell Maestre Martinez, Dr. Edward d'Auvergne, Florian Siepel, Dr. Donghan Lee, Korvin Walter und David Ban für zahlreiche Diskussion danken. 
Ein riesen Dankeschön an alle Korrekturleser!!!

Dem gesamten Arbeitskreis danke ich für die freundliche, angenehme Arbeitsatmosphäre und die allgemeine Hilfsbereitschaft.

André, Alex, Venita, Saskia und Manuel danke ich für die tolle Zeit in Göttingen und die vielen gemeinsamen Unternehmungen.

Von ganzem Herzen möchte ich mich bei meinen Eltern, meiner Schwester und meinen Großeltern bedanken. Dafür, dass sie mich immer unterstützt haben, mir Mut gemacht haben und immer an mich geglaubt haben.

Mirjam...danke, für so viele kleine und große Dinge... 


\section{Inhaltsverzeichnis}

Zusammenfassung $\quad$ v

Summary vii

Zugehörige Publikationen $\quad$ ix

Danksagungen $\quad x$

1 Einleitung 1

1.1 Strukturbiologie . . . . . . . . . . . . . . . . . 1

1.2 Ubiquitin . . . . . . . . . . . . . . . . . 2

1.3 Tagging von Proteinen . . . . . . . . . . . . . . . . . . 4

1.4 Zielsetzung der Arbeit . . . . . . . . . . . . 8

2 Paramagnetische NMR-Spektroskopie $\quad 9$

2.1 Residuale dipolare Kopplungen _... . . . . . . . . . . . . . . . . . 9

2.2 Pseudokontaktverschiebungen . . . . . . . . . . . . . . . . 19

2.3 Lanthanoide . . . . . . . . . . . . . . . . . . . . . . . . 22

2.4 Paramagnetische Relaxationsverstärkung . . . . . . . . . . . . . 23

3 Paramagnetische Tags $\quad 25$

3.1 Übersicht publizierter Tags . . . . . . . . . . . . . . . . . . . . 25

3.2 Entwicklung des TAHA-basierten Tags . . . . . . . . . . . . . . 33

4 Synthese $\quad 37$

4.1 Darstellung von Cys-TAHA . . . . . . . . . . . . . . . 37

4.2 Synthese von Cys-Ph-TAHA . . . . . . . . . . . . . . . . . . 46 
4.3 Herstellung der NMR-Proben . . . . . . . . . . . . . . . . . . . 49

5 Ergebnisse und Diskussion $\quad 55$

5.1 Pseudokontaktverschiebungen . . . . . . . . . . . . 55

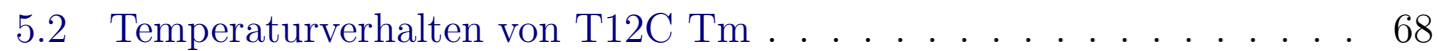

5.3 Residuale dipolare Kopplungen . . . . . . . . . . . . . . . . . 74

5.4 Vergleich der PCS- und RDC-Tensoren . . . . . . . . . . . . . 78

5.5 Zusammenfassung und Einordnung der Ergebnisse . . . . . . . . . . . 94

6 Experimenteller Teil $\quad 97$

6.1 Material und Methoden . . . . . . . . . . . . . . . . . . 97

6.2 Charakterisierung der Substanzen . . . . . . . . . . . . . 98

6.3 Protein-NMR . . . . . . . . . . . . . . . . . . . . . 99

6.4 Synthesevorschriften . . . . . . . . . . . . . . . . 103

6.5 Präparation von getaggtem, beladenem Ubiquitin . . . . . . . . . . 115

$\begin{array}{lr}\text { Literaturverzeichnis } & 117\end{array}$

$\begin{array}{ll}\text { Abbildungsverzeichnis } & 133\end{array}$

$\begin{array}{ll}\text { Tabellenverzeichnis } & 137\end{array}$

$\begin{array}{ll}\text { Abkürzungsverzeichnis } & 139\end{array}$

$\begin{array}{lll}7 & \text { Anhang } & 143\end{array}$

$\begin{array}{ll}\text { Lebenslauf } & 187\end{array}$ 


\section{Kapitel 1}

\section{Einleitung}

\section{$1.1 \quad$ Strukturbiologie}

Um das Leben auf molekularer Ebene begreifen zu können, ist der Zusammenhang von Struktur und Funktion biologischer Makromoleküle in der Strukturbiologie von großem Interesse.

Für die Strukturaufklärung sind die Röntgenstrukturanalyse und NMR-Spektroskopie die Methoden der Wahl. Mit Hilfe der Röntgenstrukturanalyse wurden bislang annähernd 60000 Strukturen gelöst und in der Proteindatenbank (PDB) hinterlegt. Darunter finden sich auch sehr große Komplexe mit einem Gewicht bis in den unteren MDa-Bereich, wie beispielsweise die große ribosomale 50S Untereinheit aus dem Archaeon Haloarcula marismortui (Ban et al. (2000)). Für ein funktionelles Verständnis ist eine statische Kristallstruktur jedoch in der Regel nicht ausreichend. Durch die Entwicklung dreidimensionaler NMR-Methoden (Griesinger et al. (1987); Oschkinat et al. (1988); Ikura et al. (1990)) können heutzutage Strukturen monomerer Proteine von 30-50 kDa standardisiert aufgeklärt werden.

Mit Hilfe der NMR-Spektroskopie können Proteine oder Oligonukleotide in ihrer nativen Konformation unter annähernd physiologischen Bedingungen gemessen werden. Ein herausragender Unterschied zur Röntgenstrukturanalyse besteht darin, dass mittels NMR-Spektroskopie Einblicke in die Dynamik von Systemen gewonnen werden können. Eine besondere Bedeutung kommt hierbei den residualen dipolaren Kopplungen (residual dipolar couplings, RDCs) zu. Diese Parameter sind in isotro- 
per Lösung zu Null gemittelt. Für die Messung von RDCs bedarf es deshalb einer partiellen Ausrichtung des Teilchenensembles. Dies wird in den Abschnitten 1.3.2 und 2.1 näher behandelt. RDCs sind sensitiv für Bewegungen im Bereich von Pikobis Millisekunden und überspannen damit auch das Zeitfenster von $500 \mathrm{~ns}-500 \mu \mathrm{s}$, in welchem Relaxationsmessungen unempfindlich sind. Viele, für die Proteinfunktion wichtige dynamische Prozesse, verlaufen jedoch langsamer als die rotatorische Korrelationszeit $\tau_{c}$, welche für Ubiquitin etwa 4 ns beträgt (Lakomek et al. (2006); Meiler et al. (2003); Peti et al. (2002)). So konnten beispielsweise mit Hilfe von RDCs bis dahin verborgene Bewegungen im Proteinrückgrat von Ubiquitin identifiziert werden. Ein auf Grundlage dieser RDC-Daten entwickeltes Strukturensemble (EROS, (ensemble refinement with orientational restraints) deckt die gesamte strukturelle Heterogenität ab, die für Ubiquitin mit verschiedenen Komplexpartnern im Kristall beobachtet wurde. Als Mechanismus der molekularen Erkennung impliziert dies eine Konformationsselektion (Lange et al. (2008); Lakomek et al. (2008)).

\subsection{Ubiquitin}

Ubiquitin ist ein kleines, globuläres Protein von universeller Bedeutung für eukaryotische Organismen. In diesen ist es in allen Zellen vorhanden und dort an einer Vielzahl von regulatorischen Prozessen, wie zum Beispiel der Zellteilung oder der DNA-Reparatur, beteiligt. Ubiquitin hat eine Molekülmasse von $8.5 \mathrm{kDa}$ und besteht aus 76 Aminosäuren. Die Sequenz verblieb im Laufe der Evolution fast unverändert. So unterscheidet sich die humane Aminosäuresequenz in lediglich drei Aminosäureresten von der Hefe Saccharomyces cerevisiae. Die humane Ubiquitinsequenz im Einbuchstabenkode lautet:

\section{MQIFVKTLTG KTITLEVEPS DTIENVKAKI QDKEGIPPDQ QRLIFAGKQL EDGRTLSDYN IQKESTLHLV LRLRGG}

Die Aminosäurereste Threonin 12 und Serin 57 sind farbig markiert, da innerhalb der vorliegenden Arbeit Cystein-Mutanten dieser Reste (T12C und S57C) verwen- 
det wurden. In Abbildung 1.1 ist die Ubiquitinstruktur im Bändermodell (A), sowie im Kalottenmodell (B) gezeigt. Ubiquitin weist zwei prägnante Sekundärstrukturelemente auf. Neben der großen $\alpha$-Helix (T22-G35) findet sich ein $\beta$-Faltblatt bestehend aus vier einzelnen Strängen. Die letzten drei Aminosäuren ragen aus der globulären Form des Proteins heraus. Neben Glycin 76 sind die beiden Lysine 48 und 63 von besonderer Bedeutung für die Funktion des Proteins. Ubiquitin bindet über G76 kovalent an das Zielprotein. Über die Lysine können weitere Ubiquitinmoleküle angebunden werden. Ketten bestehend aus mindestens fünf Ubiquitinen, die über K48 miteinander verknüpft sind, fungieren als Erkennungssignal für das Proteasom. Im Proteasom werden Proteine in die einzelnen Aminosäuren zerlegt, weshalb die Markierung durch Ubiquitin plakativ auch als kiss of death bezeichnet wird. Für ihre Arbeiten zum Proteinabbau wurden Aaron Ciechanover, Avram Hershko und Irwin Rose mit dem Nobelpreis für Chemie 2004 ausgezeichnet (Hershko et al. (1980); Hershko \& Ciechanover $(1992,1998)$ ). Die große wissenschaftliche Bedeutung von Ubiquitin zeigt sich in der Anzahl an Literaturstellen. Eine Datenbanksuche mit SciFinder lieferte über 55000 Treffer (siehe Abbildung $1.2(\mathrm{~A})$ ). Aber auch in der NMR-Spektroskopie ist Ubiquitin häufig Gegenstand der Forschung (siehe Abbildung $1.2(\mathrm{~B})$ ), da es sich aus verschiedenen Gründen hervorragend als Testprotein eignet. Zum einen lässt es sich mit hohen Ausbeuten von etwa $25 \mathrm{mg} \mathrm{L} \mathrm{L}^{-1}$ in isotopenmarkiertem Minimalmedium gut exprimieren. Zum anderen ist Ubiquitin
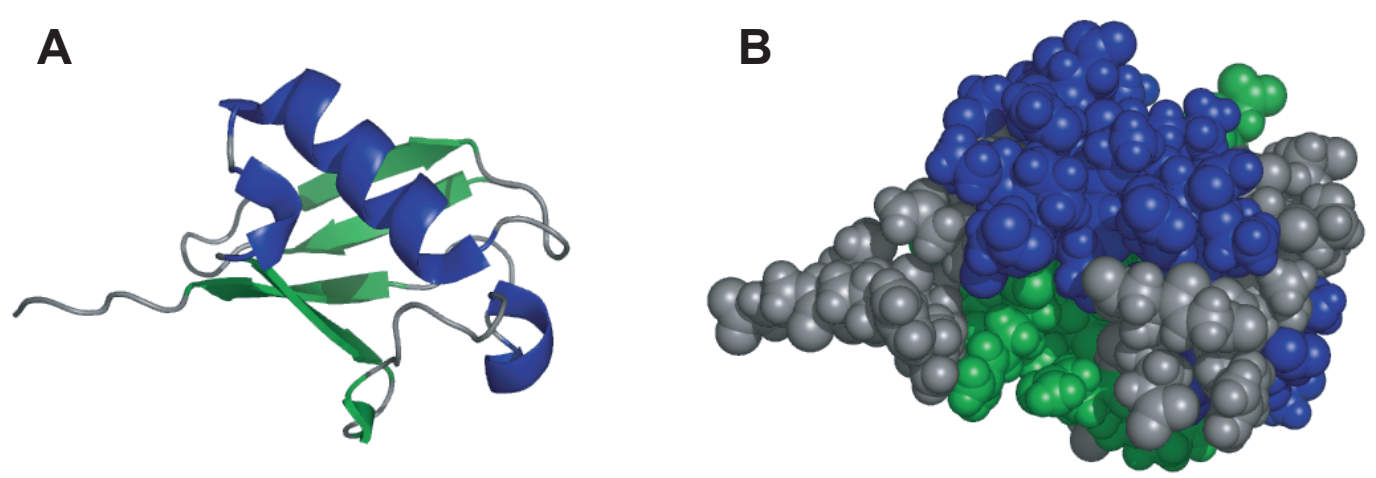

Abb. 1.1: Schematische Darstellung von Ubiquitin (PDB-Kode: 1D3Z, Cornilescu et al. (1998)) im Bänder- (A) sowie Kalottenmodell (B). Dabei sind die $\alpha$-helicalen Bereiche blau und die $\beta$-Faltblätter grün gezeigt. 

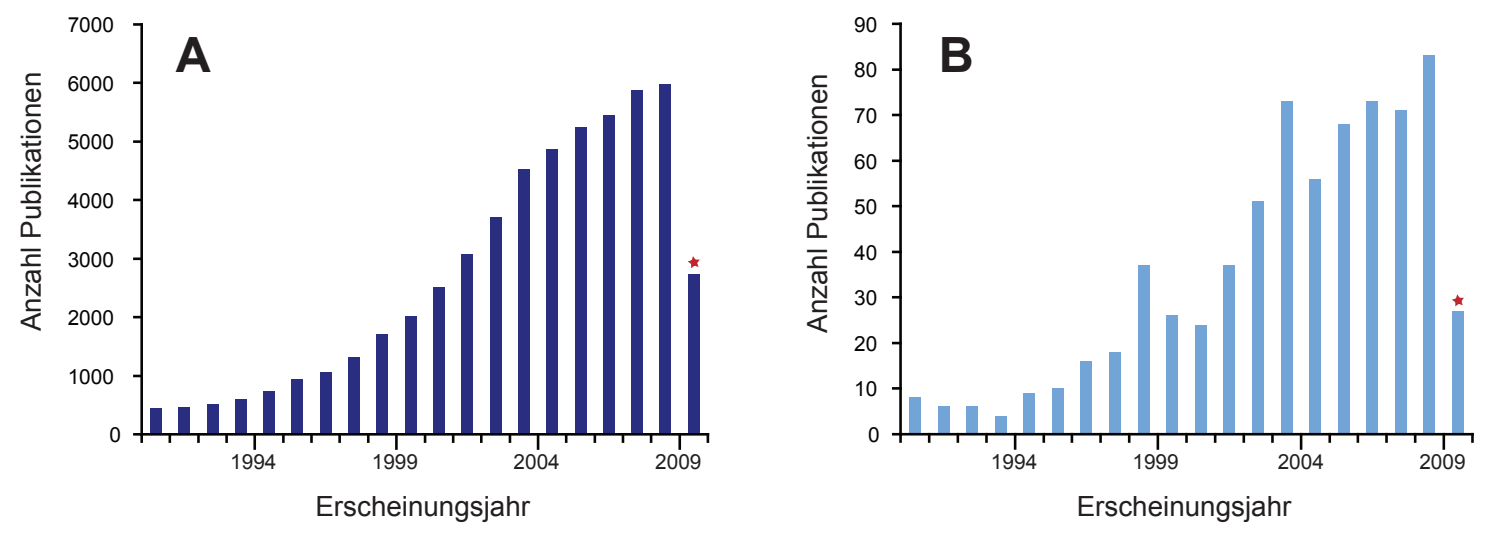

Abb. 1.2: Histogramme der Literaturstellen über Ubiquitin der letzten zwanzig Jahre. Mit dem Suchbegriff „Ubiquitin“ wurden per SciFinder-Suche insgesamt 55084 (A), für „Ubiquitin“ in Kombination mit „NMR“ 729 Einträge in der Literatur gefunden (B). Stand: 28.06.2010.

ausgesprochen stabil und toleriert weite $\mathrm{pH}$ - und Temperaturbereiche, sowie diverse Puffersysteme. Darüber hinaus sind in ${ }^{1} \mathrm{H},{ }^{15} \mathrm{~N}-\mathrm{HSQC}$-Spektren bei Raumtemperatur die Amidsignale des Proteinrückgrats nahezu perfekt aufgelöst, wodurch sich die Spektren einfach zuordnen lassen. Veränderungen im Spektrum, die durch die Einführung eines paramagnetischen Tags induziert werden, lassen sich ebenfalls gut nachvollziehen.

\subsection{Tagging von Proteinen}

Als Tagging wird die kovalente Modifikation eines Biomoleküls bezeichnet. Dabei wird eine Markierung, der so genannte Tag, am zu untersuchenden Molekül angebracht, deren Eigenschaften im Folgenden ausgenutzt werden können. Eine wichtige Anforderung an den angebrachten Tag ist, dass Funktion und Aktivität des Proteins nicht, beziehungsweise nur sehr wenig, beeinflusst werden. Die Funktionalisierung erfolgt dabei in der Regel an ausgewählten Stellen. Verschiedene Tags wurden für die unterschiedlichsten Zwecke entwickelt. Im Folgenden sind einige Beispiele angegeben. 


\subsubsection{Ausgewählte Beispiele}

Ein bekanntes Beispiel aus der Zellbiologie ist das Green Fluorescent Protein (GFP), welches erstmals aus der Qualle Aequorea victoria isoliert wurde (Shimomura et al. (1962); Tsien (1998)). Wird das GFP-Gen mit dem Zielprotein fusioniert, lässt sich anschließend die Exprimierung, sowie die räumliche und zeitliche Verteilung des markierten Proteins innerhalb einer Zelle mittels Fluoreszenzspektroskopie verfolgen. Für die Entdeckung und Weiterentwicklung des GFP wurden Osamu Shimomura, Martin Chalfie und Roger Tsien 2008 mit dem Nobelpreis für Chemie ausgezeichnet. Ein weiteres bedeutendes Beispiel ist der so genannte His-Tag für die vereinfachte Aufreinigung von Proteinen (Schmitt et al. (1993)). Am C- oder N-Terminus der Aminosäuresequenz werden sechs aufeinander folgende Histidine angefügt. Dieses Hexamotiv weist eine hohe Affinität $\mathrm{zu} \mathrm{Ni}^{2+}$-Ionen auf. Medien, wie NickelNTA Agarose, halten das getaggte Protein mit mikromolarer Affinität zunächst am Säulenmaterial zurück. Durch spätere Elution mit Imidazol wird dann das Protein wieder freigesetzt.

Stabile Radikale, wie der MTSSL-Tag (siehe Abbildung 1.3) finden in der NMRSpektroskopie Anwendung. Aminosäuren, die sich räumlich nahe am Radikal befinden, sind durch die abstandsabhängige, paramagnetische Verstärkung der Relaxation (PRE, paramagnetic relaxation enhancement) nicht mehr, beziehungsweise schwächer, detektierbar, was letztendlich Rückschlüsse auf die Tertiärstruktur des untersuchten Proteins zulässt (Liang et al. (2006); Bayrhuber et al. (2008); Lindfors et al. (2008)). Näheres zur PRE wird im Abschnitt 2.4 beschrieben. Derartige spin label werden darüber hinaus auch in der ESR-Spektroskopie (ElektronenspinresonanzSpektroskopie) verwendet. Da hier nur ungepaarte Elektronen detektiert werden

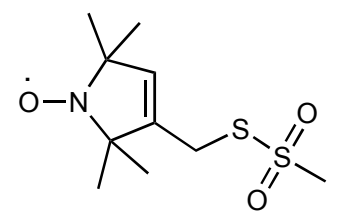

Abb. 1.3: MTSSL-Tag zur paramagnetischen Markierung von Proteinen. 
können, erweitern diese Tags die Anwendbarkeit der Methode auf Proteine, die keine oder nur eine Metallbindestelle aufweisen.

\subsubsection{Paramagnetisches Tagging}

Bei der in der vorliegenden Arbeit verwendeten Taggingmethode wird ein Metallchelator kovalent am Protein verankert. Diese Chelatfunktion kann mit einem Lanthanoidion als paramagnetische Quelle beladen werden. Der wichtigste Unterschied zwischen spin label-Tags und paramagnetischen Ionen besteht in deren magnetisch anisotroper Suszeptibilität. Die Suszeptibilität beschreibt die Magnetisierbarkeit von Materie in einem externen Magnetfeld. Ist diese anisotrop, so erfolgt eine partielle Orientierung entlang des Feldes. Dieses ist in Abbildung 1.4 schematisch dargestellt. Wenn die Verbindung, der so genannte Linker, zwischen Chelator und Protein hinreichend rigide ist, so erfährt die NMR-Probe in ihrer Gesamtheit eine partielle Ausrichtung. Dipolare Kopplungen, die in isotroper Lösung durch Gleichverteilung, sowie Brown'sche Diffusion herausgemittelt sind, werden messbar. Dies ist für die Strukturaufklärung besonders wertvoll, da dipolare Kopplungen Informationen über Winkel zwischen Interspinvektoren enthalten (Otting (2010); Su \& Otting (2010); Pintacuda et al. (2006); Bertini \& Luchinat (1999)). Zusätzlich kann mit Hilfe paramagnetischer Lanthanoid-Tags die Dynamik von Proteinen mit mehreren Domänen
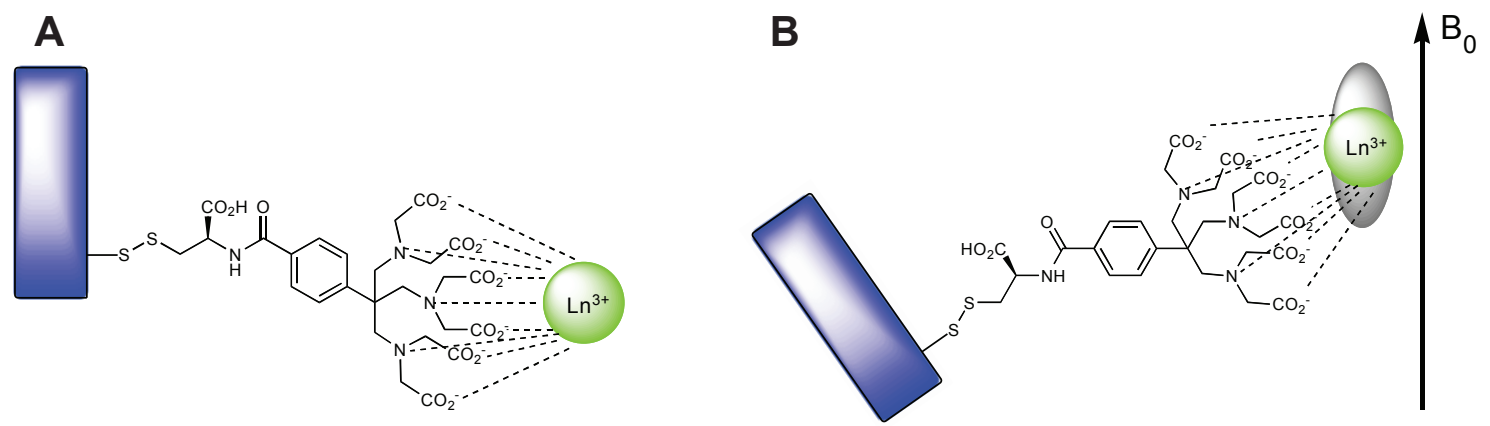

Abb. 1.4: (A) zeigt schematisch ein paramagnetisch markiertes Protein (blau). Wird dieses in ein externes Magnetfeld eingebracht (B), so erfolgt eine partielle Ausrichtung entlang $\mathrm{B}_{0}$, bedingt durch die magnetisch anisotrope Suszeptibilität des Ions (dargestellt durch den grauen Ellipsoiden). 
(Rodriguez-Castañeda et al. (2006)) oder auch Protein-Protein-Komplexen (Xu et al. (2009)) untersucht werden. Neben den RDCs werden durch die Anwesenheit des paramagnetischen Ions auch Pseudokontaktverschiebungen (PCSs) induziert. Die Interaktion mit dem ungepaarten Elektron führt zu einer Änderung der chemischen Verschiebung, die auf Grund ihrer Abstands- und Winkelabhängigkeit für die Strukturaufklärung ebenfalls sehr von Nutzen ist. Auf die paramagnetische NMRSpektroskopie, sowie die Eigenschaften der Lanthanoide wird in Kapitel 2 eingegangen.

Die Verbindung von Tag und Protein erfolgt, wie auch bei den spin label-Tags, spezifisch über eine Disulfidbrücke. Demzufolge wird für die selektive Markierung ein einzelnes, Lösungsmittel-exponiertes Cystein benötigt. Die Seitenkette des Cysteins sollte dabei vom Protein weg zeigen und möglichst frei zugänglich sein. Das Cystein wird, sofern nicht natürlich vorhanden, durch Punktmutation einer Aminosäure eingeführt. Für die Entwicklung der site-directed mutagenesis wurde 2003 der Nobelpreis für Chemie an Kary B. Mullis und Michael Smith verliehen.

Für paramagnetische Tags gelten einige spezielle Anforderungen. Idealerweise ist der Tag klein gegenüber dem Protein, um Einflüsse auf Struktur und Dynamik zu minimieren. Darüber hinaus muss der Chelator so gestaltet sein, dass er das Lanthanoidion mit hoher Affinität zu binden vermag, ohne gleichzeitig diastereomere Komplexe auszubilden. Dieser wichtige Punkt wird in Kapitel 3 näher behandelt. 


\subsection{Zielsetzung der Arbeit}

Ziel der Arbeit war es, einen neuen paramagnetischen Tag als Werkzeug für die NMR-Spektroskopie zu entwickeln und diesen zu synthetisieren. In der Vergangenheit wurden im Arbeitskreis bereits diverse EDTA-basierte Tags synthetisiert und an verschiedenen Proteinen angebracht. Die erhaltenen RDCs waren jedoch relativ klein und dementsprechend fehlerbehaftet. Zudem wichen selbst die größeren, experimentell bestimmten Werte mitunter stark von den berechneten ab. Dies wurde auf Interaktionen zwischen geladenen Aminosäureresten anderer, beziehungsweise des eigenen Proteinmoleküls mit den freien Koordinationsstellen am Lanthanoidion zurück geführt. Anstelle von EDTA sollte ein neuer Ligand gefunden werden, der die Koordinationssphäre von Lanthanoidionen möglichst vollständig absättigt und diese ungewollten Interaktionen unterbindet (siehe Kapitel 3). Gleichzeitig können größere induzierte Effekte von höher koordinierten Lanthanoidionen erwartet werden. Besonders im Hinblick auf dynamische Untersuchungen sind RDCs größer als $10 \mathrm{~Hz}$ von Interesse.

Die Eigenschaften des Tags sollten anhand zweier Ubiquitin-Cysteinmutanten und verschiedenen Lanthanoidionen charakterisiert und die jeweiligen RDCs und PCSs bestimmt werden. Darüber hinaus sollte eine verlässliche Methode gefunden werden, vollständig beladene, getaggte Proben zu erhalten, was bislang nicht reproduzierbar gelang.

Zuletzt sollten die Tensoren der RDCs und PCSs berechnet und miteinander verglichen werden. Diese wiesen zumeist große Unterschiede zueinander auf, da die Tensoren nicht in gleicher Weise durch Bewegungen des Tags beeinflusst werden. Hier sollte eine Möglichkeit gefunden werden, die Tensoren miteinander vergleichen zu können. 


\section{Kapitel 2}

\section{Paramagnetische}

\section{NMR-Spektroskopie}

\subsection{Residuale dipolare Kopplungen}

Neben der skalaren $J$-Kopplung gibt es in Form der dipolaren Kopplung $D$ eine weitere Interaktion zwischen zwei Kernen. Während $J$-Kopplungen über Molekülorbitale übertragen werden, wirken die dipolaren Kopplungen durch den Raum. Die Größe von $D$ ist unter anderem abhängig vom Abstand der koppelnden Kerne und vom Winkel $\theta$ des Interspinvektors $\vec{R}$ zum Magnetfeld $\vec{B}$ (siehe Abbildung 2.1). Auf Grund dieser Winkelabhängigkeit sind die dipolaren Kopplungen in isotroper Lösung durch die Gleichverteilung über alle Orientierungen zu Null gemittelt. In der Festkörper-NMR sind sie dagegen derart dominant (im Bereich von $\mathrm{kHz}$ ), dass sie durch Rotieren der Probe im so genannten magischen Winkel von $\theta=54.74^{\circ}$ entfernt werden müssen (MAS, magic angle spinning). Wenn die Moleküle in der Probe im zeitlichen Durchschnitt eine leichte Vorzugsorientierung einnehmen, wird die dipolare Kopplung nicht mehr vollständig herausgemittelt und es bleibt eine Restkopplung messbar. Diese wird als residuale dipolare Kopplung (residual dipolar coupling, RDC) bezeichnet und ist, abhängig vom Grad der Ausrichtung (Alignment), üblicherweise in der Größenordnung von J-Kopplungen. Eine partielle Ausrichtung der Probe kann durch externe Orientierungsmedien, wie flüssigkristalline Phasen (Sanders et al. (1994); Bax \& Tjandra (1997); Hansen et al. (1998); Ottiger et al. (1998); 


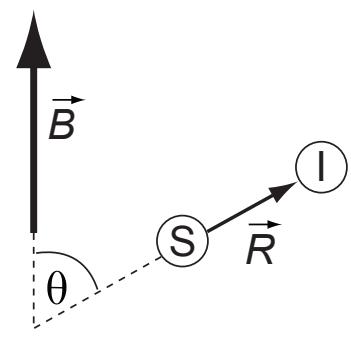

Abb. 2.1: Definition des Winkels $\theta$ zwischen dem Interspinvektor $\vec{R}$ und dem Vektor des statischen Magnetfeldes $\vec{B}$.

Prestegard et al. (2000); Peti et al. (2002); Rückert \& Otting (2000) und Thiele \& Berger (2003)), oder gestreckte beziehungsweise gestauchte Gele erfolgen (Tycko et al. (2000); Haberz et al. (2005); Kobzar et al. (2005); Kummerlöwe et al. (2007)). Abhängig vom Alignmentmedium, werden durch externe Ausrichtung teilweise dipolare Kopplungen von mehreren hundert $\mathrm{Hz}$ gemessen. Die Auswertung dieser Spektren ist durch die Dominanz der dipolaren Interaktion, aber auch durch verstärkte Relaxationseffekte erschwert. Deshalb wird versucht, die Größe der RDCs gezielt einzustellen (Sanders \& Prestegard (1990); Bax \& Tjandra (1997); Hansen et al. (1998); Marx \& Thiele (2009)). Am besten eignen sich RDCs in der Größenordnung der skalaren Kopplungskonstante.

Bei dem Ansatz des internen Alignments richten sich die Moleküle auf Grund der magnetisch anisotropen Suszeptibilität gebundener Metallionen im Magnetfeld aus. Eine der ersten RDC-Messungen wurde von Tolman et al. (1995) an eisenhaltigem Cyano-Metmyoglobin durchgeführt. Neben weiteren Untersuchungen an Metalloproteinen (Allegrozzi et al. (2000); Arnesano et al. (2000); Bertini et al. (2003); Xu et al. (2008)) wurden verschiedene paramagnetische Tags entwickelt, um Proteine, die kein Metallzentrum aufweisen, im Magnetfeld ausrichten zu können (siehe Kapitel 3). Der Vorteil des internen Alignments gegenüber externer Ausrichtung liegt darin, dass die relativen Bewegungen von Proteindomänen nicht beeinflusst werden. Die Domänendynamik kann direkt aus der Skalierung des Tensors der ungetaggten im Vergleich zu dem Tensor der getaggten Domäne abgelesen werden (Tüchelmann et al. (1998); Bertini et al. (2004); Rodriguez-Castañeda et al. (2006)). Derartige Informationen 
sind mit externen Alignmentmedien nicht zugänglich.

Um die gemessenen RDCs auswerten zu können, muss der so genannte Alignmenttensor bekannt sein. Kramer et al. (2004) haben dieses Schlüsselkonzept auf intuitive Weise in graphischer Form dargestellt. Die Ableitung der RDCs lehnt sich im Folgenden an diese Arbeit an. Zunächst wird dabei die dipolare Interaktion im Festkörper beschrieben. Anschließend wird der Alignmenttensor eingeführt und letztendlich die Gleichung der RDCs in ihrer gebräuchlichen Form gezeigt.

Der Vektor zwischen den beiden Spins $I$ und $S$ sei gegeben durch

$$
\vec{R}=r \vec{r}
$$

wobei $r$ den Abstand beider Kerne und $\vec{r}$ den Einheitsvektor in Richtung $\vec{R}$ beschreibt. In gleicher Weise kann der Vektor des statischen Magnetfeldes $\vec{B}$ durch

$$
\vec{B}=B \vec{b}
$$

ausgedrückt werden. Die Größe des Magnetfeldes wird durch $B$ gegeben während $\vec{b}$ den Einheitsvektor in Richtung $\vec{B}$ angibt. Per Konvention zeigt $\vec{B}$ im orthogonalen Laborkoordinatensystem $x^{L}, y^{L}, z^{L}$ in Richtung der $z^{L}$-Achse. Im heteronuklearen Fall, wo die Kopplungen stets schwach sind, wird der sekuläre Teil des Hamiltonoperators der Spins $I$ und $S$ durch

$$
\hat{H}_{D}=2 \pi D \hat{I}_{z^{L}} \hat{S}_{z^{L}}
$$

in Einheiten der Kreisfrequenz $\omega$ gegeben. Die dipolare Kopplungskonstante $D$ wird wie folgt beschrieben:

$$
D=\frac{\kappa}{r^{3}}\left(\cos ^{2} \theta-\frac{1}{3}\right) .
$$

Der Winkel $\theta$ ist in Abbildung 2.1 definiert. Der Term

$$
\kappa=-\frac{3}{8 \pi^{2}} \gamma_{I} \gamma_{S} \mu_{0} \hbar
$$


besteht ausschließlich aus physikalischen Konstanten, wie den gyromagnetischen Verhältnissen $\gamma_{I}$ und $\gamma_{S}$ der Spins $I$ und $S$, der Permeabilitätskonstante des Vakuums $\mu_{0}$ und dem reduzierten Planck'schen Wirkungsquantum $\hbar=h / 2 \pi$. Für das Spinpaar ${ }^{15} \mathrm{~N}-{ }^{1} \mathrm{H}$ beträgt $\kappa=36.5 \mathrm{kHz} \AA^{3}$. Damit ergibt sich im Festkörper eine maximale dipolare Kopplung $D_{\max }=21.7 \mathrm{kHz}$ für $\cos ^{2} \theta=1$ und einer $\mathrm{NH}$ Bindungslänge von $r=1.04 \AA$ in Einheiten der Frequenz $\nu$.

A

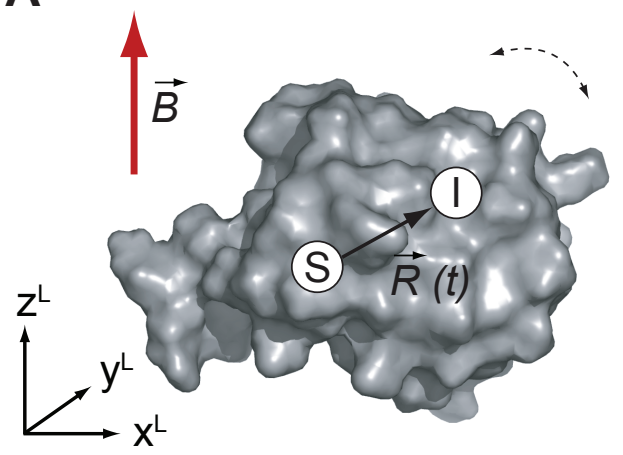

B

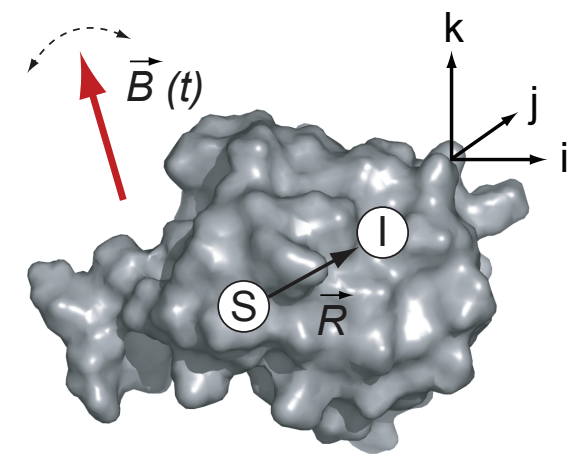

Abb. 2.2: Einfluss der molekularen Reorientierung in Lösung. Aus Sicht des Laborkoordinatensystems $x^{L}, y^{L}, z^{L}$ ergibt sich ein zeitabhängiger Interspinvektor $\vec{R}(t)$ (A). Durch den Wechsel in das Molekül-fixierte Koordinatensystem i, j, k wird bei konstantem $\vec{R}$ der zeitabhängige Magnetfeldvektor $\vec{B}(t)$ erhalten (B).

Wenn sich das Molekül in Lösung reorientiert, wird der Interspinvektor $\vec{R}$ zeitabhängig und somit auch der Winkel $\theta$ zwischen $\vec{R}$ und dem Laborkoordinatensystem, beziehungsweise $\vec{B}$. Dem zufolge ändert sich auch der $\cos ^{2} \theta$-Term und letztendlich die dipolare Kopplung $D$ in Abhängigkeit von der Zeit (siehe Abbildung $2.2 \mathrm{~A}$ ). Die zeitlich gemittelte Konstante der dipolaren Kopplung $\overline{D_{R D C}}$ wird als residuale dipolare Kopplung bezeichnet:

$$
\overline{D_{R D C}}=\frac{\kappa}{r^{3}}\left(\overline{\cos ^{2} \theta}-\frac{1}{3}\right) .
$$

Die Größe ist abhängig von der Stärke des Alignments. Die gemessene dipolare Kopplung wird über das Teilchenensemble gemittelt. Da die Bevorzugung bestimmter Orientierungen gegenüber anderen nur im Promillebereich stattfindet, wird die dipolare Kopplung aus dem kHz-Bereich bis auf wenige $\mathrm{Hz}$ herunter skaliert. 
Für die Ableitung des Alignmenttensors A wird das Bezugssystem vom Laborkoordinatensystem hin zu einem beliebigen orthogonalen Molekülkoordinatensystem i, j, k gewechselt. Unter der Annahme, dass das Molekül keine interne Dynamik aufweist, bleibt $\vec{R}$ in diesem Koordinatensystem konstant. Stattdessen ergibt sich, wie in Abbildung 2.2 B gezeigt, ein zeitabhängiger Magnetfeldvektor

$$
\vec{B}(t)=B \vec{b}(t)
$$

Innerhalb des Molekülkoordinatensystems lässt sich die Verteilung für $\vec{B}(t)$ graphisch in Form eines Ellipsoiden darstellen (Abbildung 2.3 A). Der Wahrscheinlichkeitstensor

$$
\mathbf{P}=\left(\begin{array}{ccc}
\overline{b_{i}^{2}(t)} & \overline{b_{i}(t) b_{j}(t)} & \overline{b_{i}(t) b_{k}(t)} \\
\overline{b_{j}(t) b_{i}(t)} & \overline{b_{j}^{2}(t)} & \overline{b_{j}(t) b_{k}(t)} \\
\overline{b_{k}(t) b_{i}(t)} & \overline{b_{k}(t) b_{j}(t)} & \overline{b_{k}^{2}(t)}
\end{array}\right)
$$

ist eine reale, symmetrische $3 \times 3$ Matrix mit einer Spur von 1. $\mathbf{P}$ gibt die Häufigkeit an, mit der ein Magnetfeldvektor in einer bestimmten Orientierung anzutreffen ist. Durch eine weitere Rotation des Koordinatensystems in das Hauptachsensystem von $\mathbf{P}$ kann die Matrix diagonalisiert werden (Abbildung $2.3 \mathrm{~B}$ ). Innerhalb dieses besonderen molekülfixierten Koordinatensystems lassen sich die RDCs einfacher berechnen. Die diagonalisierte Matrix

$$
\mathbf{P}=\left(\begin{array}{ccc}
P_{x} & 0 & 0 \\
0 & P_{y} & 0 \\
0 & 0 & P_{z}
\end{array}\right)
$$

hat eine Spur von 1, wobei gilt:

$$
P_{x} \leq P_{y} \leq P_{z}
$$



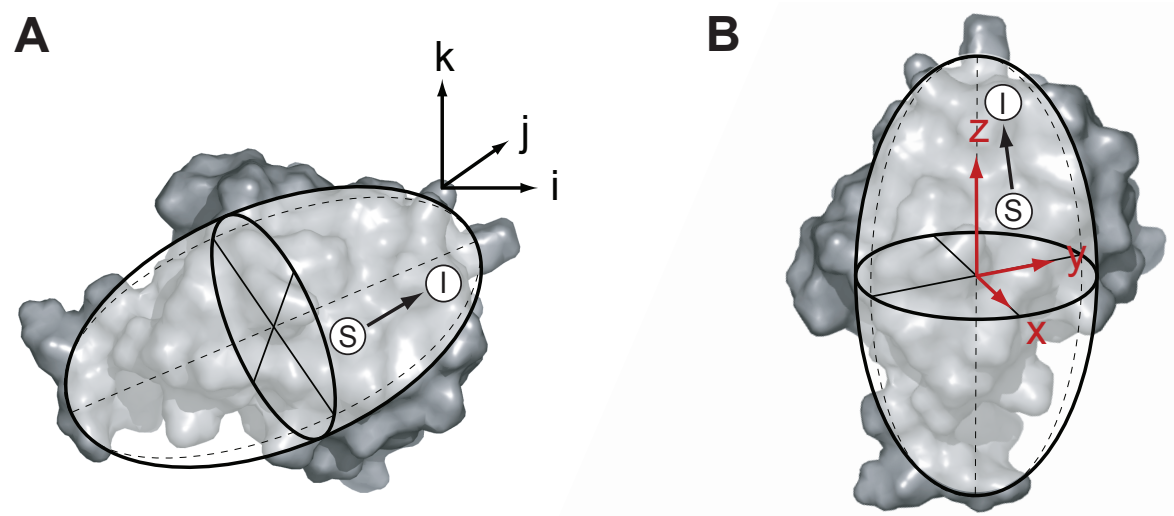

Abb. 2.3: Graphische Darstellung des Wahrscheinlichkeitstensors $\mathbf{P}$ (grauer Ellipsoid) im Molekülkoordinatensystem (A). Durch die Rotation in das Hauptachsensystem x, y, z von $\mathbf{P}$ (B), lassen sich die RDCs einfacher berechnen.

Für einen beliebigen internuklearen Vektor, dessen kartesische Komponenten $r_{x}, r_{y}$ und $r_{z}$ in diesem Koordinatensystem bekannt sind, kann der Term $\overline{\cos ^{2} \theta}$ wie folgt ausgedrückt werden:

$$
\overline{\cos ^{2} \theta}=P_{x} r_{x}^{2}+P_{y} r_{y}^{2}+P_{z} r_{z}^{2}
$$

Wird dieser Ausdruck in die Gleichung 2.6 eingesetzt, können bereits RDCs berechnet werden. In der Literatur wird allerdings in der Regel an Stelle des Wahrscheinlichkeitstensors der bereits erwähnte Alignmenttensor A verwendet. Dieser wird durch die Differenz von $\mathbf{P}$ mit der Einheitskugel gegeben:

$$
\mathbf{A}=\mathbf{P}-\frac{1}{3} \mathbf{1}
$$

Da die Hauptachsen identisch sind, lassen sich die Komponenten folgendermaßen darstellen:

$$
A_{x}=P_{x}-\frac{1}{3}, \quad A_{y}=P_{y}-\frac{1}{3}, \quad A_{z}=P_{z}-\frac{1}{3}
$$

Umgeformt und in Gleichung 2.11 eingesetzt ergibt sich daraus

$$
\left(\overline{\cos ^{2} \theta}-\frac{1}{3}\right)=A_{x} r_{x}^{2}+A_{y} r_{y}^{2}+A_{z} r_{z}^{2}
$$


A

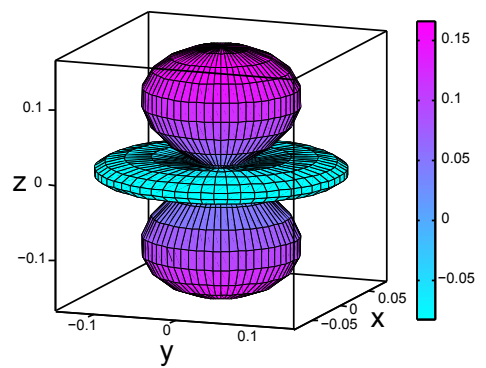

B

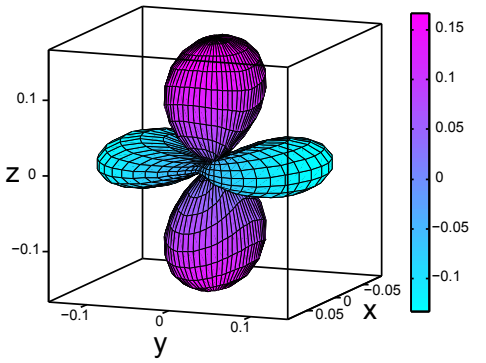

C

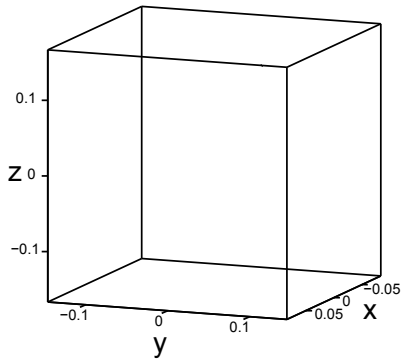

Abb. 2.4: Graphische Darstellung verschiedener Alignmenttensoren. (A) korrespondiert $\mathrm{zu}$ einem axialsymmetrischen Wahrscheinlichkeitstensor mit $\mathrm{P}_{x}=\mathrm{P}_{y}=0.25$ und $\mathrm{P}_{z}=0.5$, während (B) sich auf einen rhombischen Ellipsoiden zurückführen lässt mit $\mathrm{P}_{x}=0.2, \mathrm{P}_{y}=0.3$ und $\mathrm{P}_{z}=0.5$. Im Fall $(\mathrm{C})$ liegt ein isotroper Wahrscheinlichkeitstensor mit $\mathrm{P}_{x}=\mathrm{P}_{y}=\mathrm{P}_{z}=1 / 3$ zu Grunde und A mittelt sich zu Null.

Zusammen mit Gleichung 2.6 werden die RDCs

$$
\overline{D_{R D C}}=\frac{\kappa}{r^{3}}\left(A_{x} r_{x}^{2}+A_{y} r_{y}^{2}+A_{z} r_{z}^{2}\right)
$$

erhalten. Da der Alignmenttensor A im Gegensatz zu $\mathbf{P}$ spurlos ist, kann dieser nicht durch einen Ellipsoiden dargestellt werden. Allerdings sind die Orientierungswahrscheinlichkeiten durch eine Linearkombination des Polarteils der $d$-Funktionen darzustellen. In Abbildung 2.4 sind repräsentativ verschiedene Alignmenttensoren gezeigt, die zu einem axialsymmetrischen (A), rhombischen (B), beziehungsweise sphärischen (C) Wahrscheinlichkeitstensor korrespondieren. Aus dem Alignmenttensor lassen sich Größe und Vorzeichen der RDCs entnehmen. In der Literatur finden sich diverse äquivalente Beschreibungen der Gleichung für die dipolaren Kopplungen, die über entsprechende Faktoren ineinander umgerechnet werden können. Bertini et al. (2002) haben diese verschiedenen Gleichungen zusammengestellt. Bei der Orientierung der Probe mittels paramagnetischer Ionen besteht eine quadratische Abhängigkeit der RDCs vom externen Magnetfeld $\mathrm{B}_{0}$, die über den D-Tensor eingeführt wird. Darüber hinaus wird der Zusammenhang zur so genannten SaupeMatrix (Saupe (1968); Losonczi et al. (1999)) gegeben. Diese verbindet die Tensorkoordinaten mit dem externen Magnetfeld. Letztendlich wird die RDC-Gleichung 


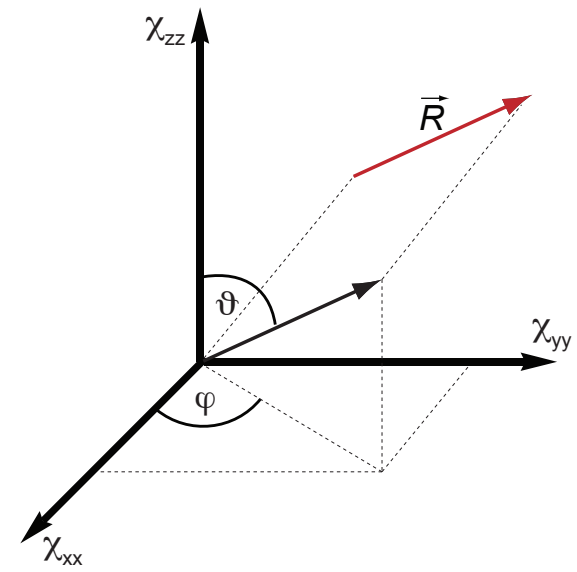

Abb. 2.5: Definition der Polarwinkel $\vartheta$ und $\varphi$ für einen beliebigen internuklearen Vektor $\vec{R}$ im Koordinatensystem des $\chi$-Tensors.

in ihrer gebräuchlichsten Form in Abhängigkeit von $\chi$, dem Tensor der magnetisch anisotropen Suszeptibilität, in Verbindung mit den Polarkoordinaten $\vartheta$ und $\varphi$ angegeben (siehe Abbildung 2.5). In dieser Form ähnelt sie stark der Gleichung für die PCSs.

Im Folgenden wird die Umwandlung von Gleichung 2.15 gezeigt. Die Komponenten des Einheitsvektors $\vec{r}$ lassen sich in Polarkoordinaten in folgender Form ausdrücken:

$$
\vec{r}=\left(\begin{array}{c}
r_{x} \\
r_{y} \\
r_{z}
\end{array}\right)=\left(\begin{array}{c}
\sin \vartheta \cos \varphi \\
\sin \vartheta \sin \varphi \\
\cos \vartheta
\end{array}\right)
$$

Eingesetzt in Gleichung 2.14 ergibt sich unter Ausnutzung der trigonometrischen Identitäten $\cos ^{2} \varphi=(1+\cos 2 \varphi) / 2$ und $\sin ^{2} \varphi=(1-\cos 2 \varphi) / 2$ :

$$
\begin{array}{r}
\left(\overline{\cos ^{2} \theta}-\frac{1}{3}\right)=\frac{A_{x}}{2} \sin ^{2} \vartheta+\frac{A_{x}}{2} \sin ^{2} \vartheta \cos 2 \varphi \\
+\frac{A_{y}}{2} \sin ^{2} \vartheta-\frac{A_{y}}{2} \sin ^{2} \vartheta \cos 2 \varphi \\
+\frac{A_{z}}{2} \cos ^{2} \vartheta
\end{array}
$$


Da $\mathbf{A}$ spurlos ist, gilt $A_{x}+A_{y}=-A_{z}$ und zusammen mit $\sin ^{2} \vartheta=1-\cos ^{2} \vartheta$ lässt sich die Gleichung umformen zu:

$$
\left(\overline{\cos ^{2} \theta}-\frac{1}{3}\right)=\frac{A_{z}}{2}\left(3 \cos ^{2} \vartheta-1\right)+\frac{A_{x}-A_{y}}{2} \sin ^{2} \vartheta \cos 2 \varphi
$$

wobei die Terme $A_{z} / 2$ und $\left(A_{x}-A_{y}\right) / 2$ die axiale, beziehungsweise rhombische Komponente des Alignmenttensors beschreiben. Die Saupematrix $\mathbf{S}$ steht mit dem Alignmenttensor in folgendem Zusammenhang:

$$
\mathbf{S}=\frac{3}{2} \mathbf{A}
$$

Über die Beziehungen

$$
D_{a x}=\frac{1}{3}\left(S_{z z}-\frac{S_{x x}+S_{y y}}{2}\right)=\frac{S_{z z}}{2}
$$

und

$$
D_{r h}=\frac{S_{x x}-S_{y y}}{3}
$$

ergeben sich zusammen mit

$$
D_{a x}=\frac{1}{2} \frac{B_{0}^{2}}{15 \mu_{0} k T} \Delta \chi_{a x}, \quad D_{r h}=\frac{1}{2} \frac{B_{0}^{2}}{15 \mu_{0} k T} \Delta \chi_{r h}
$$

(Bertini et al. (2002)) und Gleichung 2.18 eingesetzt in Gleichung 2.6 die RDCs in Abhängigkeit vom $\chi$-Tensor:

$$
\overline{D_{R D C}}=-\frac{B_{0}^{2} \gamma_{I} \gamma_{S} \hbar}{8 \pi^{2} r^{3} 15 k T}\left[\Delta \chi_{a x}\left(3 \cos ^{2} \vartheta-1\right)+\frac{3}{2} \Delta \chi_{r h} \sin ^{2} \vartheta \cos 2 \varphi\right]
$$

$\Delta \chi_{a x}$ und $\Delta \chi_{r h}$ bezeichnen die axiale, beziehungsweise rhombische Komponente des symmetrischen $\chi$-Tensors der magnetischen Suszeptibilität des Metallions. Sie werden durch die folgenden Definitionen gegeben:

$$
\Delta \chi_{a x}=\chi_{z z}-\frac{\chi_{x x}+\chi_{y y}}{2}=\frac{3}{2} \chi_{z z}
$$


und

$$
\Delta \chi_{r h}=\chi_{x x}-\chi_{y y}
$$

Die Gleichung 2.23 gibt die RDCs in ihrer gebräuchlisten Form an. Alternative Beschreibungen und Herleitungen finden sich in der Literatur (Bax et al. (2001); Prestegard et al. (2000) sowie Bertini et al. (2002)). Da A, S und $\boldsymbol{\chi}$ spurlose, symmetrische Matrizen sind, reichen für deren eindeutige Beschreibung theoretisch fünf RDCs aus, so lange deren korrespondierende internukleare Vektoren eine hinreichende Winkelstreuung, genauer Orthogonalität, im fünfdimensionalen Raum aufweisen. In der Praxis werden allerdings deutlich mehr Werte für eine ausreichend genaue Bestimmung des Tensors benötigt. 


\subsection{Pseudokontaktverschiebungen}

Wenn sich ein paramagnetisches Metallion in der Nähe eines Kerns aufhält, so wird die chemische Verschiebung des Kerns durch eine dipolare, durch den Raum vermittelte Interaktion mit dem Spin des ungepaarten Elektrons beeinflusst. Die dipolare Kopplung zwischen dem Kern und dem Elektron führt zu einer Dublettaufspaltung des Signals. Auf Grund der schnellen longitudinalen Relaxationszeit des Elektrons (meist im ps-Bereich oder noch schneller), ist jedoch nur der Mittelwert dieser Kopplung zu sehen. Im Fall des Elektrons ist der $\beta$-Zustand auf Grund des großen gyromagnetischen Verhältnisses des Elektrons deutlich stärker populiert als der $\alpha$-Zustand, so dass sich, bedingt durch die Mittelung der Kopplung, insgesamt eine Verschiebung der Resonanzfrequenz des Kerns ergibt. Diese Verschiebung ist proportional zur dipolaren Kopplung zwischen Kern und Elektron, sowie zu der Besetzungsdifferenz des $\alpha$ - und $\beta$-Niveaus des Elektrons. Die Differenz der chemischen Verschiebungen, bezogen auf eine isotrope Referenzprobe, wird als Pseudokontaktverschiebung (pseudocontact shift, PCS) bezeichnet. Abhängig vom Abstand zum Ion ergeben sich deutliche PCSs in den Spektren in einer Größenordnung von bis zu mehreren ppm. Die PCSs werden durch folgende Gleichung beschrieben:

$$
\delta^{P C S}=\frac{1}{12 \pi r_{M I}^{3}}\left[\Delta \chi_{a x}\left(3 \cos ^{2} \Theta-1\right)+\frac{3}{2} \Delta \chi_{r h} \sin ^{2} \Theta \cos 2 \Phi\right]
$$

wobei $\Theta$ und $\Phi$ die Polarwinkel im $\chi$-Tensor zum Abstand $r_{M I}$ beschreiben (siehe Abbildung 2.6). Ein wichtiger Unterschied zu den RDCs liegt darin, dass im Fall der PCS $r_{M I}$ den Abstand zwischen dem Metallion und dem betrachteten Kern angibt. Näherungsweise wird hierbei ein metallzentrierter Punktdipol angenommen, da zum einen die Verteilung der Spindichte über den Raum in der Regel nicht bekannt ist und zum anderen bei Metallionen davon ausgegangen werden kann, dass sich ungepaarte Elektronen nahe des Kerns aufhalten (McConnell \& Robertson (1958); Bleaney (1972); Bertini et al. (2002)).

Für das Auftreten der PCSs ist keine partielle Ausrichtung der Probe erforderlich. Zusätzlich zum Suszeptibilitätstensor geht der Abstand zwischen dem Elektron und 


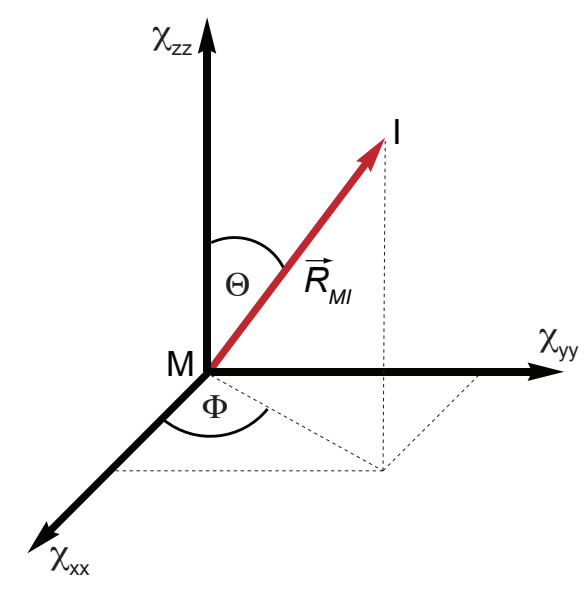

Abb. 2.6: Definition der Polarwinkel $\Theta$ und $\Phi$ im Koordinatensystem des $\chi$-Tensors. Der Vektor $\vec{R}_{M I}=r_{M I} \vec{r}$ zeigt vom Metallion zum betrachteten Kern, wobei $r_{M I}$ den Abstand angibt.

dem Kern mit $r_{M I}^{-3}$ in die Größe der Pseudokontaktverschiebungen ein. Graphisch lässt sich dieser Zusammenhang in Form von PCS-Isoflächen darstellen (siehe Abbildung 2.7, Pintacuda et al. $(2004,2007))$. Kerne, die auf der Isofläche liegen, weisen eine bestimmte PCS auf. Je näher diese Kerne am Metall liegen, desto größer wird dessen Einfluss auf die chemische Verschiebung. Die Kerne, die im sogenannten magischen Winkel liegen $\left(\Theta=54.74^{\circ}\right)$, werden nicht beeinflusst.

In der Literatur werden, wie auch in dieser Arbeit, die PCSs in der Regel gemäß Gleichung 2.26 ausgewertet. Allerdings soll an dieser Stelle erwähnt werden, dass für die Einfachheit der Gleichung einige Näherungen in Kauf genommen werden. Bedingt durch die magnetische Anisotropie und die resultierende partielle Ausrichtung der Probe, wird die Gleichung um einen, vom Magnetfeld abhängigen, Term erweitert. Für einen axialsymmetrischen Suszeptibilitätstensor ergibt sich:

$$
\delta_{a x}^{P C S, o r}=\frac{1}{12 \pi r_{M I}^{3}} \Delta \chi_{a x}\left(3 \cos ^{2} \Theta-1\right)\left[1+\Delta \chi_{a x} \frac{B_{0}^{2}}{15 \mu_{0} k T}\right]
$$

Da aber, wie bei der Einführung der RDCs erwähnt, nur etwa jedes tausendste bis zehntausendste Molekül ausgerichtet ist (Alignmenttensoren sind im Promillebereich), ist dieser Einfluss zu vernachlässigen. 


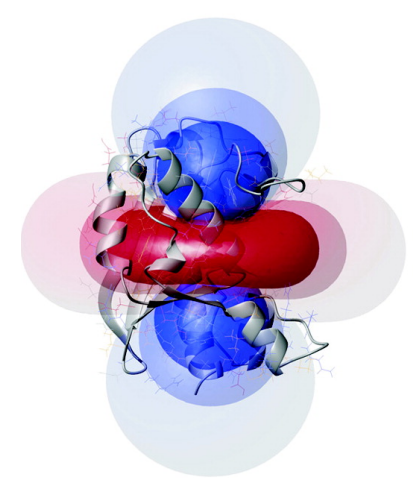

Abb. 2.7: Darstellung der Isoflächen gleicher PCSs. Die Abbildung wurde aus Pintacuda et al. (2007) entnommen. Gezeigt sind die Dysprosium-induzierten Isoflächen für $\pm 3, \pm 1.5$ und $\pm 0.5 \mathrm{ppm}$, zusammen mit der Proteinstruktur von $\epsilon 186$. Bereiche mit positiven PCSs sind blau, die mit negativen rot dargestellt.

Die Pseudokontaktverschiebungen hängen, wie gezeigt, von der Anisotropie der magnetischen Suszeptibilität und somit gemäß des Curie-Gesetzes reziprok von der Temperatur ab. Im Fall von Lanthanoidionen müssen für die exakte Beschreibung der PCSs, auf Grund der starken Spin-Bahn-Kopplung, durch Reihenentwicklung noch weitere Terme von $\mathrm{T}^{-n}$ mit einbezogen werden (Bleaney (1972); McGarvey (1979)). Unter der Annahme, dass sich die Molekülgeometrie in Lösung nicht mit der Temperatur oder der Anzahl an Elektronen im 4f-Orbital ändert, weisen die PCSs näherungsweise eine Temperaturabhängigkeit von $\mathrm{T}^{-2}$ auf.

Ein zweiter paramagnetischer Effekt auf die chemische Verschiebung wird durch die so genannte Fermi-Kontaktwechselwirkung oder Kontaktverschiebung beschrieben (Kurland \& McGarvey (1970)). Diese entsteht durch das ungepaarte Elektron, dessen Anwesenheit die Spindichte und somit die Resonanzfrequenz benachbarter Kerne ändert. Die Kontaktverschiebung ist über einige Bindungen von Relevanz, hat jedoch bei der Verwendung paramagnetischer Tags keinen Einfluss mehr auf das Protein, da zu viele Bindungen zwischen dem Ion und den beobachteten Kernen liegen. 


\subsection{Lanthanoide}

Als Lanthanoide (Ln) oder Seltenerden werden im Periodensystem die $f$-Block Elemente von Lanthan bis Lutetium bezeichnet. Der Begriff Seltenerden ist dabei etwas irreführend, da zum Beispiel Cer häufiger natürlich auftritt als Blei oder Arsen. Ihr Anteil an der Erdkruste beträgt insgesamt 0.02\%. Streng genommen ist Lanthan als Element des $d$-Blocks kein Lanthanoid, wird aber allgemein, auf Grund dessen Eigenschaften, hinzu gezählt. Die Lanthanoide haben eine Elektronenkonfiguration von $[\mathrm{Xe}] 6 s^{2} 4 f^{n}$ und sind alle in der Oxidationsstufe + III stabil. Die frühen Elemente der Periode bevorzugen in Lösung eine neunfache Koordination $\left(\left[\mathrm{Ln}\left(\mathrm{H}_{2} \mathrm{O}\right)_{9}\right]^{3+}\right)$. Der Wechsel auf eine achtfache Koordination $\left[\mathrm{Ln}\left(\mathrm{H}_{2} \mathrm{O}\right)_{8}\right]^{3+}$ erfolgt bei Samarium (Djanashvili et al. (2008)).

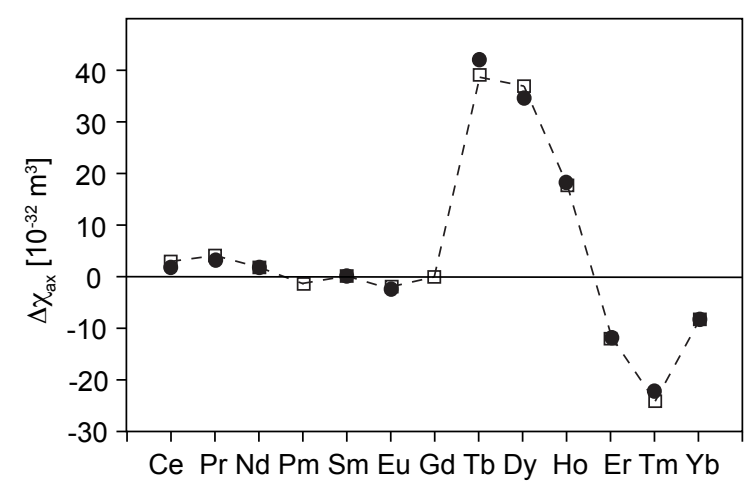

Abb. 2.8: Experimenteller (gefüllte Kreise) und berechneter (Quadrate) Verlauf der $\Delta \chi_{a x}$-Werte für die Reihe der Lanthanoide, gemessen an Calbindin $\mathrm{D}_{9 k}$. Die Abbildung wurde aus Bertini et al. (2001) entnommen und auf Grund der mangelhaften Auflösung nachbearbeitet.

Bei den Lanthanoiden wird mit den $4 f$-Orbitalen nur die drittäußerste Schale aufgefüllt. Die freien Elektronen werden durch die weiter außen liegenden $4 d$ - und $5 p$ Orbitale abgeschirmt, so dass die paramagnetische Relaxationsverstärkung deutlich geringer ist, als bei den Übergangsmetallen. Die $f$-Orbitale selbst sind stark anisotrop, was besonders bei $\mathrm{Tb}^{3+}$ und $\mathrm{Dy}^{3+}$ zu großen axialen Komponenten der magnetischen Suszeptibilität und einer großen Ausrichtung im Magnetfeld führt (siehe Abbildung 2.8). Eine weitere Eigenschaft der Lanthanoide ist ihr äußerst ähnliches chemisches Verhalten. Somit können die Ergebnisse, wenn ein paramagnetischer Tag 
mit verschiedenen Lanthanoidionen beladen wurde, direkt miteinander verglichen werden. Auf Grund dieser Eigenschaften sind diese Elemente für die paramagnetische NMR-Spektroskopie von besonderem Interesse und werden seit mehr als 40 Jahren eingesetzt (Sanders \& Williams (1970); Barry et al. (1971); Bleaney (1972)). Eine Übersicht der aktuellen Literatur findet sich in Otting (2010, 2008).

\subsection{Paramagnetische Relaxationsverstärkung}

Das gyromagnetische Verhältnis freier Elektronen $\gamma_{e}$ ist 658-fach größer als das von Protonen. Da die transversale Magnetisierung unter anderem über Spin-SpinWechselwirkungen in Richtung des Gleichgewichtszustands abgebaut wird, haben ungepaarte Elektronen einen erheblichen Einfluss auf die transversale Relaxationszeit $T_{2}$. Dieses Phänomen wird als paramagnetische Relaxationsverstärkung (PRE, paramagnetic relaxation enhancement) bezeichnet. Die verkürzte transversale Relaxation resultiert in einer Verbreiterung der Peaks, wobei $\Delta \nu_{1 / 2}$ die Linienbreite auf halber Höhe angibt:

$$
\frac{1}{T_{2}}=\pi \Delta \nu_{1 / 2}
$$

Wenn die rotatorische Korrelationszeit $\tau_{c}$ deutlich größer, als die Elektronenrelaxationszeit $\tau_{e}$ ist, wird die Relaxation hauptsächlich durch die Curie-Spin-Relaxation beeinflusst (Gueron (1975)). Die longitudinale Elektronenrelaxationszeit aller $\mathrm{Ln}^{3+}$ Ionen ist im Bereich von 10 ${ }^{-13} \mathrm{~s}$ (Pintacuda et al. (2007); Alsaadi et al. (1980)). Eine Ausnahme ist das $\mathrm{Gd}^{3+}$-Ion, bei dem jedes der sieben $f$-Orbitale mit einem ungepaarten Elektron besetzt ist. Die deutlich längere $\tau_{e}$ von $10^{-7} \mathrm{~s}$ erklärt sich dadurch, dass die Symmetrie erst durch Lösungsmitteleffekte gebrochen werden muss (Hudson \& Lewis (1970)). Die Kernrelaxation, induziert durch die Wechselwirkung des magnetischen Moments des Elektrons und des Kernspins bei der Rotation des Moleküls im Magnetfeld, wird als Curie-Spin-Relaxation bezeichnet. Es gilt folgende Proportionalität (Otting (2008)):

$$
R_{2, C}^{P R E} \propto \frac{\gamma^{2} B_{0}^{2}\left[g_{e} S_{e}\left(S_{e}+1\right)\right]^{2} \tau_{c}}{r^{6} T^{2}},
$$


wobei $g_{e}$ und $S_{e}$ den $g$-Faktor, beziehungsweise den Spin des Elektrons angeben. Es lassen sich einige Rückschlüsse aus Gleichung 2.29 ziehen. Der PRE-Effekt ist abstandsabhängig mit $\mathrm{r}^{-6}$ und enthält somit strukturelle Informationen. Darüber hinaus ist die Relaxationsverstärkung für ${ }^{13} \mathrm{C}$ und ${ }^{15} \mathrm{~N}$ geringer als für ${ }^{1} \mathrm{H}$ auf Grund der quadratischen Proportionalität zum gyromagnetischen Verhältnis.

Resonanzen der Kerne, die nahe des paramagnetischen Ions liegen, werden so stark verbreitert, dass sie nicht mehr detektierbar sind. Dies wird in der Literatur als bleaching area bezeichnet und erklärt, weshalb die Spektren paramagnetischer Proben stets weniger Signale enthalten. Deshalb überwiegen die negativen Aspekte in Form von Signalverbreiterung und -verlust in der Regel den zusätzlichen Informationsgehalt. Daher ist es ein Parameter bei den Tags, die Ionen möglichst weit entfernt vom Protein zu positionieren, so dass die PRE gering ist, aber dennoch PCSs beobachtet werden. Für die paramagnetisch induzierten RDCs ist dies nicht entscheidend, da hier keine Abstandsabhängigkeit zum Metall besteht. Weitere Effekte, wie die Curie-dipolare kreuzkorrelierte Relaxation (Hus et al. (2000)), wurden in dieser Arbeit nicht betrachtet und werden daher nicht diskutiert. 


\section{Kapitel 3}

\section{Paramagnetische Tags}

\section{1 Übersicht publizierter Tags}

\subsubsection{EDTA-basierte Tags}

In der Literatur wurden bereits einige Tags auf der Basis von EDTA beschrieben. Diese dienten zunächst allerdings nicht der Messung paramagnetischer NMR-Effekte. Vielmehr wurden die mit Eisen(II) beladenen Tags zur ortsspezifischen Hydrolyse von Peptiden oder RNA eingesetzt. Durch Zugabe von Wasserstoffperoxid bilden sich eisenkatalysiert Hydroxylradikale in der Nähe des Eisenzentrums (Fenton-Reaktion). Aus den erhaltenen Fragmenten können Rückschlüsse auf Strukturen und Interaktionsflächen von Protein-Protein- beziehungsweise Protein-Oligonukleotid-Komplexen gezogen werden (Ebright et al. (1992); Ermacora et al. (1992); Datwyler \& Meares (2000)). Mit S-(2-Pyridylthio)cysteaminyl-EDTA (siehe Abbildung 3.1) wur-<smiles>O=C(O)CN(CCN(CC(=O)O)CC(=O)O)CC(=O)O</smiles>

Abb. 3.1: $S$-(2-Pyridylthio)cysteaminyl-EDTA, kommerziell erhältlich bei Toronto Research Chemicals. 
den erstmals Proteine mittels eines chelatisierenden Tags ausgerichtet (Tüchelmann et al. (1998); Gaponenko et al. (2002); Dvoretsky et al. (2002)). Zur Ausrichtung entlang des Magnetfeldes wurden hier allerdings die Übergangsmetalle Cobalt(II) und Mangan(II) verwendet. Die erhaltenen PCSs (bis zu 0.3 ppm) und RDCs (bis zu $4 \mathrm{~Hz}$ ) sind relativ klein, allerdings wurde gezeigt, dass das Konzept des internen Alignments mittels Tags funktioniert.<smiles>CS(=O)(=O)SCCNC(=O)CN(CCN(CC(=O)O)CC(=O)O)CC(=O)O</smiles><smiles>CS(=O)(=O)Sc1ccc(NC(=O)[C@H](CN(CC(=O)O)CC(=O)O)N(CC(=O)O)CC(=O)O)cc1</smiles>

C $(R)$<smiles>CS(=O)(=O)Sc1ccc(NC(=O)[C@H](CN(CC(=O)O)CC(=O)O)N(CC(=O)O)CC(=O)O)cc1</smiles><smiles>CS(=O)(=O)SC[C@@H](NC(=O)[C@H](CN(CC(=O)O)CC(=O)O)N(CC(=O)O)CC(=O)O)C(=O)O</smiles>

$\mathbf{D}(R, S)$

Abb. 3.2: Übersicht der EDTA-basierten Tags. Der Stern in Struktur (A) markiert das pseudoasymmetrische Stickstoffatom. Um chirale Komplexe zu vermeiden, wurden (B) $-(C)$ enantiomerenrein hergestellt. Der Tag (D) wurde ebenfalls in $(R)$-Konfiguration am Chelator synthetisiert.

Im Arbeitskreis wurden EDTA-Tags mit Lanthanoidionen beladen. Diese Chelatfunktion wurde ausgewählt, da die EDTA-Lanthanoidkomplexe mit einer Dissoziationskonstante von etwa $10^{-18} \mathrm{~mol} \mathrm{~L}^{-1}$ äußerst stabil sind (Sigl \& Sigl (2003); Evans (1990)). Eine Übersicht der publizierten Tags ist in Abbildung 3.2 gegeben. Der Tag (A) wurde erhalten, indem eine der vier Carboxylfunktionen mit dem Linker über eine Amidbindung verbunden wurde. Zwar konnten mit diesem Tag wie gewünscht dipolare Kopplungen und Pseudokontaktverschiebungen induziert werden, jedoch wurden zwei Datensätze zweier unabhängiger Alignmenttensoren erhalten (Ikegami et al. (2004); Pintacuda et al. (2004)). Der Grund dafür liegt in der Komplexbildung. 


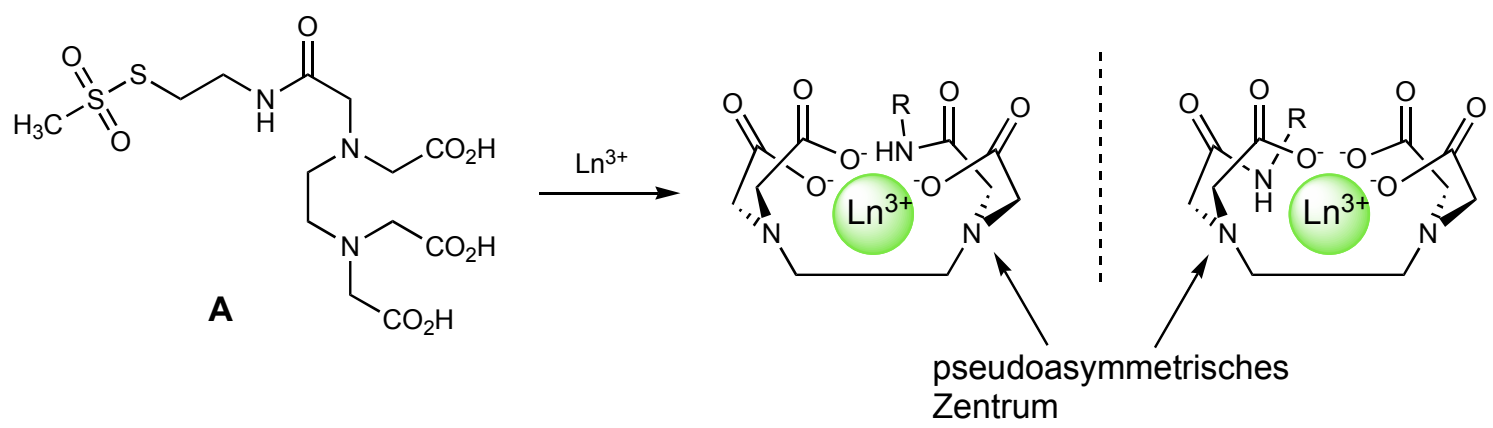

Abb. 3.3: Schematische Darstellung der diastereomeren Komplexbildung, ausgehend vom achiralen EDTA-Derivat A (Ikegami et al. (2004); Leonov et al. (2005)).

Obwohl das EDTA-Derivat selbst achiral ist, weist es dennoch ein pseudoasymmetrisches Zentrum am Stickstoff auf. Aus diesem Grund werden zwei diastereomere Komplexe geformt, was letztendlich in der Verdopplung der NMR-Signale resultiert. Eine schematische Darstellung ist in Abbildung 3.3 gezeigt.

Um stereochemisch uniforme Metallkomplexe zu erhalten, wurden die Tags (B) - (D) entwickelt, bei denen der Chelator mit definierter Konfiguration am Linker angebracht ist (Ikegami et al. (2004); Leonov et al. (2005); Haberz et al. (2006)). Ursprünglich wurde der Chelator racemisch dargestellt und anschließend per HPLC getrennt. Mittlerweile ist das Ausgangsmaterial für die Tags (B)-(D) (2,3-Diaminopropionsäure) enantiomerenrein erhältlich. Mit diesen EDTA-Tags wurden an apoCalmodulin RDCs von bis zu $8 \mathrm{~Hz}$ gemessen.

\subsubsection{DTPA- und DOTA-basierte Tags}

Neben EDTA sind einige weitere Liganden bekannt, die äußerst stabile Lanthanoidkomplexe ausbilden. DTPA oder DOTA sind achtzähnige Liganden, die das gebundene Metall nicht mehr freisetzen $\left(\mathrm{K}_{D}<10^{-20} \mathrm{~mol} \mathrm{~L}^{-1}\right)$. In deren Lanthanoidkomplexen ist die neunte Koordinationstelle von Wasser besetzt. Dieses befindet sich in schnellem Austausch mit dem Wasser des Lösungsmittels. So werden beispielsweise in der Kernspintomographie Gadoliniumkomplexe als Kontrastmittel eingesetzt, was zu einer erheblichen Relaxationssteigerung des Wassers führt. Die hohen Komplexstabilitätskonstanten verbunden mit der hohen Absättigung der 

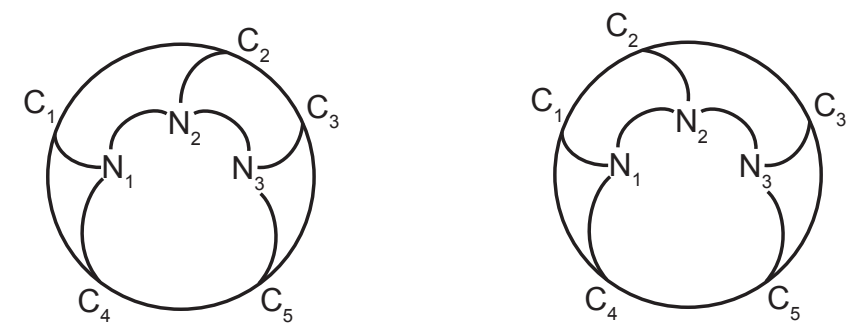

Abb. 3.4: Points-on-a-sphere-Modell der beiden enantiomeren DTPA-Konformationen im Lanthanoidkomplex (Franklin \& Raymond (1994)).

Koordinationssphäre machen diese Chelatoren grundsätzlich auch für die paramagnetische NMR-Spektroskopie interessant. Im Gegensatz zu ETDA treten allerdings schon bei den unmodifizierten Ligandkomplexen Stereoisomere auf. Die auftretende Chiralität bei DTPA-Komplexen lässt sich durch ein points-on-a-sphere-Modell erklären, welches in Abbildung 3.4 gezeigt ist. Die Lanthanoidbindung resultiert in einem Enantiomerenpaar des Komplexes, da das zentrale Stickstoffatom $\mathrm{N}_{2}$ durch die unterschiedliche Koordination der Carboxylgruppe $\mathrm{C}_{2}$ chiral wird.

Durch die Immobilisierung des DTPA-basierten CLaNP-1-Tags über zwei Linker am Protein (siehe Abbildung 3.5) wurden weitere Stereoisomere induziert, da hier neben dem Stereozentrum am zentralen Stickstoffatom noch zwei weitere Stereozentren an den anderen beiden Stickstoffatomen erzeugt wurden. Der Austausch zwischen den einzelnen Isomeren verläuft langsam auf der NMR-Zeitskala, so dass letztendlich fünf unterscheidbare Datensätze mit verschiedenen Tensoren erhalten wurden (Prudêncio et al. (2004)).<smiles>CS(=O)(=O)SCCNC(=O)CN(CCN(CC(=O)O)CC(=O)O)CCN(CC(=O)O)CC(=O)NCCSS(C)(=O)=O</smiles>

Abb. 3.5: Der DTPA-basierte CLaNP-1-Tag (Caged Lanthanide NMR Probe). 

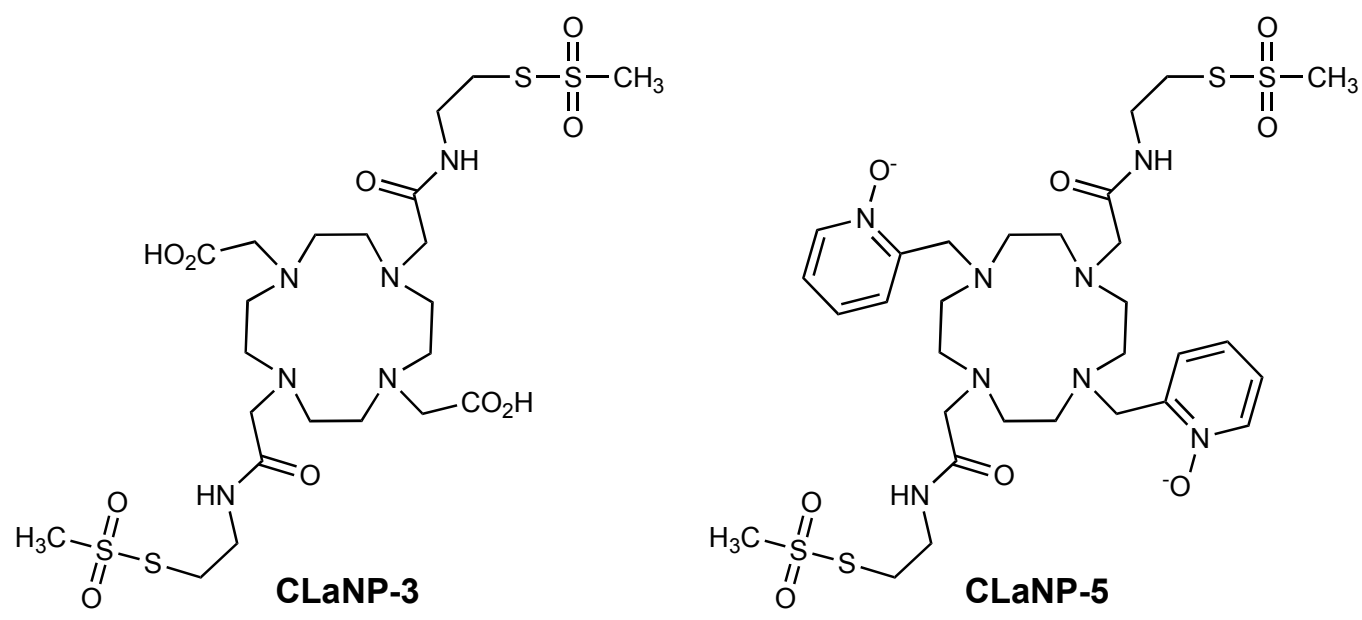

Abb. 3.6: Die DOTA-basierten Tags CLaNP-3 und CLaNP-5.

Bei DOTA erklären sich die unterschiedlichen Konformere durch das Auftreten helikaler Chiralität. Sowohl die vier Ethyleneinheiten im Ring, als auch die Acetatarme bilden eine Helix aus, deren Gang gemäß den IUPAC-Regeln mit $\Lambda / \Delta$ für die Anordnung der Carboxylgruppen und $(\lambda \lambda \lambda \lambda) /(\delta \delta \delta \delta)$ für den Ring bezeichnet werden. Somit ergeben sich zwei enantiomere Paare von Diastereomeren, die sich bei Raumtemperatur im langsamen Austausch zueinander befinden. Dieser Austausch der vier Isomere wird zusätzlich von einer Umwandlung der Konformation in Verbindung mit einer Änderung der Koordinationsphäre überlagert. Obwohl nicht alle Isomere gleich populiert sind, verkompliziert es die Analyse der NMR-Spektren weiter (Aime et al. (1997)). Für den DOTA-basierten CLaNP-3 wurden vier unterscheidbare Datensätze durch verschiedene Alignmenttensoren gefunden, wobei zwei eine deutlich höhere Population als die anderen beiden aufwiesen (Vlasie et al. (2007)). Wenn die Resonanzen zweier unabhängiger Tensoren sehr ähnlich populiert sind, so ergeben sich Probleme bei der eindeutigen Zuweisung zum jeweiligen Datensatz.

Durch Substitution zweier Carboxymethyleinheiten am DOTA-Ring gegen [(Pyridin$N$-oxid)-2yl]methyl (Polasek et al. (2004)) nimmt der Komplex nur noch eine Konformation ein. Dementsprechend wurden bei CLaNP-5-getaggten Proteinen keine Verdopplungen der Resonanzen mehr beobachtet (siehe Abbildung 3.6). Durch die Verknüpfung des mit Thulium beladenen Tags mit dem Protein über zwei Arme konnten bereits bei relativ niedrigem Feld (600 MHz) große RDCs von bis zu $25 \mathrm{~Hz}$ 

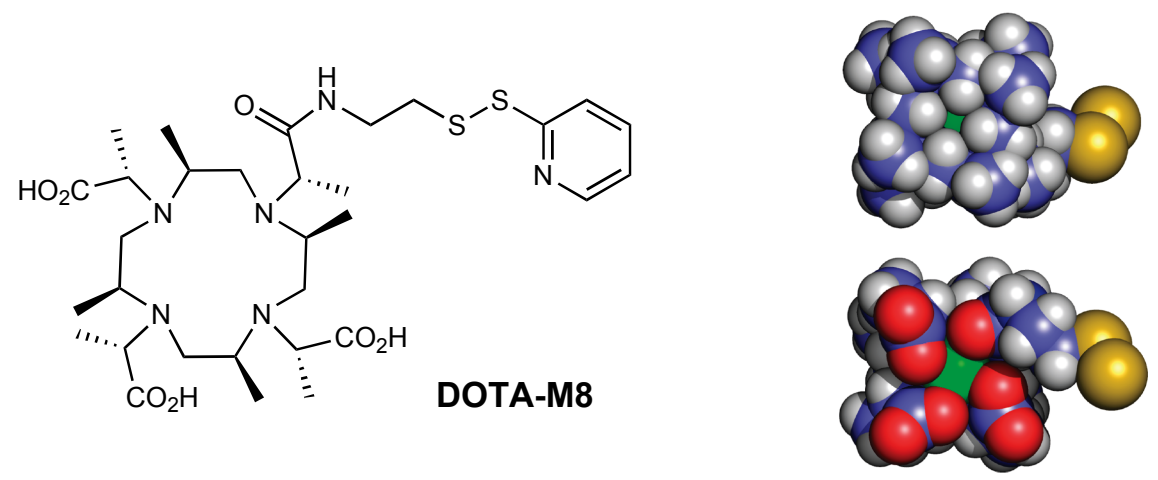

Abb. 3.7: Der DOTA-basierte DOTA-M8-Tag. Alle Stereozentren sind $(S)$-konfiguriert. Auf der rechten Seite ist eine schematische Darstellung des beladenen Komplexes im Kalottenmodell $(\Delta / \delta \delta \delta \delta)$ gezeigt.

gemessen werden (Keizers et al. (2007, 2008)). Eingeschränkt wird die praktische Anwendung jedoch durch die Tatsache, dass die Proteinstruktur bereits in hohem Maße bekannt sein muss. Zur Herstellung der erforderlichen Cystein-Doppelmutante werden zwei Lösungsmittel-exponierte Seitenketten gleicher Orientierung in geeignetem Abstand benötigt. Darüber hinaus treten bei der Taggingreaktion Tag-verbrückte Dimere auf, die anschließend abgetrennt werden müssen.

Durch die stereoselektive Einführung von Methylsubstituenten am Ring, sowie an den Carboxymethyleinheiten, entsteht ein sterisch äußerst anspruchsvoller Chelatligand. In Abhängigkeit von der Konfiguration der acht eingeführten Stereozentren weisen sowohl der Ring, als auch die substituierten Carboxymethylarme nur noch eine helicale Gangrichtung auf (Ranganathan et al. (2002)). Dieses methylsubstituierte Gerüst war die Grundlage des DOTA-M8-Tags (Häussinger et al. (2009), siehe Abbildung 3.7). Im Gegensatz zu den zuvor erwähnten Tags auf DOTA-Basis erfolgt die Verknüpfung zum Protein nur über eine Disulfidbrücke. Dennoch werden durch diesen Tag große RDCs (mit Dysprosium bis zu 20 Hz bei 800 MHz) induziert. Dies unterstreicht den sterischen Anspruch des Tags. Im Tag sind die Lanthanoidionen derart fest gebunden, dass DOTA-M8 acht Stunden bei $75{ }^{\circ} \mathrm{C}$ mit dem entsprechenden Metallsalz inkubiert werden muss, um den Tag beladen zu können. Der so vorbeladene Tag kann anschließend per HPLC aufgereinigt werden. Neben dem gewünschten, paramagnetisch verschobenen Signalsatz tritt eine weitere Spezies auf. 
<smiles>O=C(O)c1ccc(S)c(C(=O)O)n1</smiles>

3MDPA<smiles>O=C(O)c1cc(CS)cc(C(=O)O)n1</smiles>

4MMDPA<smiles>O=C(O)c1cccc(C(=O)O)n1</smiles>

DPA

Abb. 3.8: Übersicht über die DPA-basierten Tags. Der trimere DPA-Lanthanoidkomplex $\left(\left[\operatorname{Ln}(\mathrm{DPA})_{3}\right]^{3-}\right)$ wird als Verschiebungsreagenz verwendet.

Bei 298 K liegt deren Beitrag bei 15-20\%, steigt jedoch temperaturabhängig auf $50 \%$ bei 323 K. Dieser Effekt ist durch Abkühlen in einer Größenordnung von mehreren Stunden vollständig reversibel. Als mögliche Erklärung wird im Artikel die cis-trans Isomerisierung der Peptidbindung des Linkers genannt. Die Synthese von DOTA-M8 ist darüber hinaus mühsam, da bereits die Ausbeute bei der Darstellung des zu Grunde liegenden, vierfach methylsubstituierten DOTA-Rings von etwa 2\% sehr gering ist (Ranganathan et al. (2002)).

\subsubsection{DPA-basierte Tags}

Ein ganz anderer Ansatz wird in der Gruppe von Gottfried Otting verfolgt. Hier werden sehr kleine und rigide Tags auf der Basis von Dipicolinsäure (DPA) verwendet, um Lanthanoidionen zu immobilisieren. DPA ist ein dreizähniger Ligand, der eine nanomolare Affinität zu Lanthanoiden aufweist. Zur Stabilisierung des Komplexes werden jedoch zusätzlich Proteinseitenketten, wie Glutamate, in der direkten Nachbarschaft benötigt, die als Anker fungieren. In Abbildung 3.8 sind zwei dieser Tags gezeigt. 4MMDPA weist noch eine flexible Methyleneinheit auf ( $\mathrm{Su}$ et al. (2008)), die bei 3MDPA entfernt wurde (Man et al. (2010)). Beide Tags wurden nicht mit einer aktivierenden Gruppe am Schwefel versehen. Die Knüpfung der Disulfidbindung erfolgte hier durch Verwendung des Ellman's Reagenz DTNB (siehe Abbildung 3.9, Ellman (1959); Su et al. (2006)). Dabei wird DTNB vom freien Cysteinthiol angegriffen und gespalten. Auf diese Weise wird das Protein aktiviert und kann im nächsten Schritt mit dem Tag reagieren. Mit diesen Tags wurden PCSs von bis zu 2 ppm gemessen. RDCs ließen sich ebenfalls messen, jedoch waren diese mit bis zu $9 \mathrm{~Hz}$ 


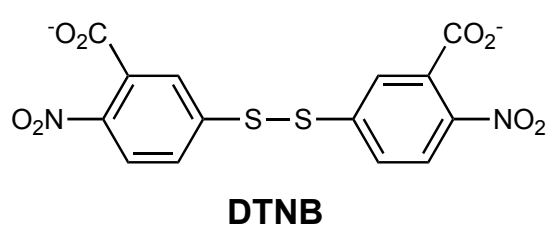

\begin{abstract}
Abb. 3.9: Ellman's Reagenz DTNB (5,5'-Dithiobis(2,2'-nitrobenzoesäure)) zur Verknüpfung thiolhaltiger Verbindungen mit Cysteinseitenketten.
\end{abstract}

für Terbium bei einem Feld von $800 \mathrm{MHz}$ kleiner als die der anderen vorgestellten Tags. Auch die Übereinstimmung mit den zurück gerechneten Daten war geringer als bei den zuvor gezeigten Tags. Eine weitere Vereinfachung der DPA-Tags stellt $\left[\operatorname{Ln}(\mathrm{DPA})_{3}\right]^{3-}$ dar. Dieser Komplex ist im eigentlichen Sinne kein Tag mehr, sondern vielmehr ein paramagnetisches Verschiebungsreagenz (Su et al. (2009)). Lanthanoide bilden mit drei Äquivalenten DPA einen $C_{3}$-symmetrischen Komplex aus. Vorsichtig zum Protein titriert, so wird es beschrieben, setzt sich dieser mit millimolarer Affinität an eine bevorzugte Position auf der Proteinoberfläche. Die Verwendung von zwei Äquivalenten des Komplexes führt dabei in der Regel nicht zur Bindung an eine zweite Stelle auf der Oberfläche.

\title{
3.1.4 Metall-bindende Proteinsequenzen
}

In der Literatur wurden neben Tags auf der Grundlage organischer Chelatoren auch spezifische Proteinsequenzen zur Metallfixierung beschrieben. Diese werden an den C- oder N-Terminus des zu untersuchenden Proteins fusioniert, allerdings muss darauf geachtet werden, dass die Struktur des Proteins nicht beeinflusst wird. In einem der ersten Ansätze wurde das Zinkfingermotiv verwendet und dieses mit Cobalt(II) beladen (Gaponenko et al. (2000)). Später wurden Sequenzen mit weniger als zwanzig Aminosäuren entwickelt, die selektiv Lanthanoidionen mit nanomolarer Affinität fixieren (Franz et al. (2003); Nitz et al. (2003); Wöhnert et al. (2003); Nitz et al. (2004)). Durch die Verwendung eines doppelten Lanthanoid-bindenden Tags, wobei jeweils eine Sequenz an den C- und N-Terminus gekoppelt wurde, konnten für Ubiquitin RDCs von bis zu -18 Hz gemessen werden. Dies entspricht einer Verdreifachung der RDCs, bezogen auf das einfach getaggte Protein (Martin et al. (2007)). 
Durch die Einführung eines Cysteins innerhalb der Lanthanoid-bindenden Peptidsequenz können diese, in Analogie zu den organometallischen Tags, an freie Cysteine geknüpft werden, wodurch die Limitierung auf den C- und N-Terminus des Proteins wegfällt (Su et al. (2008)).

Ein sehr elegantes, Lanthanoid-bindendes Konstrukt ist die N60D Calmodulinmutante (Bertini et al. (2003)). Hier wird eine weitere negative Ladung in eine der vier Calciumbindestellen eingeführt, wodurch bevorzugt Lanthanoidionen gebunden werden. Auf diese Weise wird ein internes Alignment erhalten, ohne die Struktur oder die Gesamtmasse des Proteins signifikant zu ändern. Mit dieser Mutante wurde die Dynamik des aus zwei Domänen bestehenden Calmodulins untersucht (Bertini et al. (2004)).

\subsection{Entwicklung des TAHA-basierten Tags}

Bei der Verwendung der EDTA-Tags ergaben sich in der Vergangenheit diverse Probleme. Die erhaltenen RDCs von maximal $8 \mathrm{~Hz}$ waren im Mittel relativ klein und dazu häufig mit großen Fehlern behaftet. Das langfristige Ziel des Projekts, nämlich ein Werkzeug für die NMR-Spektroskopie zu entwickeln, mit dem die Dynamik von Proteindomänen untersucht werden kann, konnte mit diesen Tags noch nicht erfüllt werden, da die dynamisch reduzierten Alignmenttensoren und damit auch die RDCs sehr klein wurden. Darüber hinaus waren die Signale negativ geladener Aminosäuren, wie Glutamate, in der Nähe des Tags zum Teil stärker verbreitert als die anderer Aminosäuren. Das legt nahe, dass diese Reste mit dem Tag entweder intramolekular oder sogar intermolekular interagiert haben. Dies lässt sich dadurch erklären, dass die EDTA-basierten Tags lediglich sechs Koordinationsstellen aufweisen und somit nicht in der Lage sind, die Koordinationssphäre von Lanthanoidionen vollständig auszufüllen (Djanashvili et al. (2008)). Um diese ungewollten Wechselwirkungen zu unterbinden, muss ein neuer Tag mit mehr als sechs Koordinationsstellen entworfen werden. Bei den vorgestellten Tags ist tendenziell ein Zusammenhang zwischen der Anzahl der Chelatarme und der Stärke des Alignments festzustellen. Inwieweit die- 
<smiles>O=C(O)CN(CC(=O)O)CC(CN(CC(=O)O)CC(=O)O)CN(CC(=O)O)CC(=O)O</smiles>

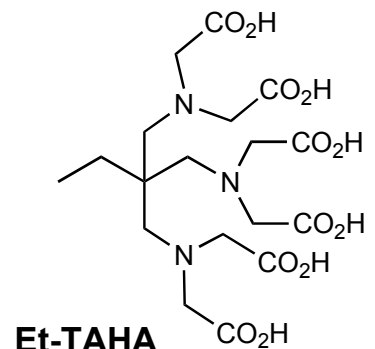

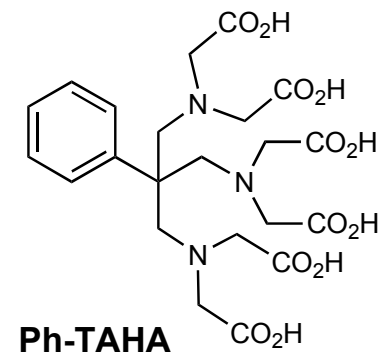

Abb. 3.10: Struktur der TAHA-Liganden.

ser Effekt rein sterischer Natur ist, da ein erhöhter Raumbedarf die Mobilität des Tags einschränkt, kann an dieser Stelle nicht geklärt werden.

Das wichtigste Kriterium für einen neuartigen Liganden ist jedoch die Vermeidung diastereomerer Komplexe. Um dies zu verhindern, muss der Chelator selbst hochsymmetrisch sein und zusätzlich so wenig flexible Fragmente wie möglich aufweisen. Auf Grund der zuvor diskutierten stereochemischen Probleme wurde der Tag nicht von DOTA oder DTPA abgeleitet. Als Chelatligand für den neuen Tag wurde TAHA (Triaminohexaacetat, siehe Abbildung 3.10) ausgewählt. Dieser neunzähnige Ligand erfüllt durch die $C_{3}$-symmetrische Achse die genannten Anforderungen, da keine Stereozentren an den Stickstoffatomen entstehen können.

Der Chelator wurde in der Literatur bislang nur wenige Male beschrieben. Erstmals wurden im Jahr 2001 Et-TAHA und Ph-TAHA im Rahmen einer Studie veröffentlicht, in der neuartige, mehrzähnige Liganden für Komplexe der Seltenerden synthetisiert und analysiert wurden (Viguier et al. (2001)). Für eine Reihe von Lanthanoid(III)-Ionen wurden die Komplexstabilitätskonstanten gemessen. Bei den frühen Elementen der Periode betragen sie $10^{12}$, für $\mathrm{Dy}^{3+}$ bereits $10^{15}$. Damit sind die TAHAKomplexe zwar um etwa drei Größenordnungen instabiler als die entsprechenden EDTA-Komplexe, aber immer noch hinreichend stabil, sodass die Komplexierung durch den Chelator nicht in Konkurrenz mit Bindung an der Proteinoberfläche tritt. In einer weiteren Veröffentlichung über Komplexbildner für die Radioimmunotherapie wurde gezeigt, dass Galliumkomplexe des Liganden (hier als TAME-Hex bezeichnet) auch in Gegenwart eines tausendfachen Überschusses an DTPA stabil sind (Arslantas et al. (2004)). Auch wenn kein direkter Rückschluss auf Lanthanoidkom- 
Tab. 3.1: Wassermoleküle in der inneren Koordinationssphäre des TAHA Liganden (Viguier et al. (2001)). Die Werte für pH 7 und 8 waren nicht angegeben.

\begin{tabular}{ccc}
\hline & $\mathrm{pH}$ & Anzahl $\mathrm{H}_{2} \mathrm{O}$ \\
\hline Ph-TAHA & 1 & 4.4 \\
& 2 & 4.2 \\
3 & 3.4 \\
4 & 1.7 \\
5 & 1.5 \\
6 & 1.4 \\
9 & 1.1 \\
\hline
\end{tabular}

plexe gezogen werden kann, so unterstreicht dieses Ergebnis dennoch die Qualität des Chelatliganden. Darüber hinaus haben Viguier et al. die Absättigung der Koordinationssphäre für Ph-TAHA mittels DIS (dysprosium induced shift, Alpoim et al. (1992)) bestimmt. Bei der Methode wird die Dysprosium-induzierte Änderung der ${ }^{17} \mathrm{O}-$ Resonanzfrequenz des Wassers gemessen (die natürliche Häufigkeit von ${ }^{17} \mathrm{O}$ beträgt $0.0373 \%$ ). Durch Variation der Konzentration an Dysprosium-Metall-Komplex können Rückschlüsse auf die Koordinationssphäre gezogen werden. Die Ergebnisse der Messungen sind in Tabelle 3.1 gezeigt. Demnach sind bei dem beabsichtigten pH-Wert von 6.8 für die NMR-Messungen weniger als 1.4 Wassermoleküle in der inneren Koordinationssphäre gebunden, was deren hohe Absättigung durch den TAHA-Liganden verdeutlicht, obgleich der Komplex, bedingt durch die sechs Säurefunktionen, dreifach negativ geladen ist. Durch die Stabilität der Metallkomplexe, verbunden mit der Anzahl an Koordinationsstellen und der hohen Symmetrie, ist der TAHA-Ligand ideal für die Verwendung als Chelatfunktion eines paramagnetischen Tags.

Zur Verknüpfung mit dem Protein wird noch ein Verbindungsstück, der so genannte Linker, benötigt. Dieser sollte möglichst klein und rigide sein, um das Alignment des Tags größtmöglich auf das Protein zu übertragen. Gleichzeitig darf der Abstand zwischen Protein und Tag nicht zu kurz sein, damit die Taggingreaktion durch den 
voluminösen Metallkomplex sterisch nicht gehindert wird. Für den Linker wurde (S)-Mesylcystein (Weidner \& Block (1972); Hart (1985)) ausgewählt, um eine Vergleichbarkeit mit den EDTA-Tags zu gewährleisten, beziehungsweise Unterschiede in der Ausrichtung der Probe ausschließlich auf den neuartigen Chelator zurückführen zu können. 


\section{Kapitel 4}

\section{Synthese}

\subsection{Darstellung von Cys-TAHA}

Wie in Kapitel 3.2 beschrieben, war es ein Ziel der vorliegenden Arbeit, einen neuen Tag zur paramagnetischen Markierung von Proteinen auf Basis des TAHA-Liganden zu synthetisieren. Bei der Syntheseplanung musste eine funktionelle Gruppe für die spätere Verknüpfung mit dem Protein an der $C_{3}$-Achse eingeführt werden. In Analogie zu den EDTA-basierten Tags wurde hierzu eine Carboxylfunktion gewählt, da der Tag auf diese Weise über eine Amidbindung mit verschiedenen Linkern verbunden und somit flexibel eingesetzt werden kann. Beispielsweise wurde im Arbeitskreis die Modifikation von Oligonukleotiden mittels Clickchemie (Wöltjen (2009)) demonstriert, was eine Azidfunktion im Linker erfordert. Um den Abstand zwischen Protein und Metallion möglichst kurz zu halten, wurde Cys-TAHA (Cysteinyl-TAHA) als Zielmolekül ausgewählt (siehe Abbildung 4.1). Die verschiedenen Säurefunktionen<smiles>CS(=O)(=O)SC[C@@H](NC(=O)C(CN(CC(=O)O)CC(=O)O)(CN(CC(=O)O)CC(=O)O)CN(CC(=O)O)C(=O)O)C(=O)O</smiles>

Abb. 4.1: Zielmolekül der ersten Synthesen: Cys-TAHA. 
des Zielmoleküls Cys-TAHA erforderten eine orthogonale Schutzgruppenstrategie. Die Carboxylfunktion, über die die spätere Verbindung mit dem Biomolekül erfolgt, sollte als Benzylester geschützt werden. Benzylester lassen sich durch Palladiumvermittelte Hydrogenolyse (Chiu et al. (1995)) effektiv entfernen. Die übrigen sechs Carboxylfunktionen sollten als säurelabile tert-Butylester dargestellt werden.

\subsubsection{Erster Syntheseansatz}

Kommerziell sind keine geeigneten Triamine mit einer zusätzlichen funktionellen Gruppe erhältlich. Dementsprechend musste dieses Grundgerüst neu aufgebaut werden. Für den ersten Syntheseansatz (siehe Abbildung 4.2) wurde Pentaerythrit 1 als günstiges Edukt gewählt. Zunächst wurde eine der vier Hydroxylgruppen selektiv mit tert-Butyldiphenylsilylchlorid geschützt (Hanessian \& Lavallee (1975)). Diese Schutzgruppe wurde aus zwei Gründen ausgewählt. Zum einen ließ sich 2, trotz der drei Hydroxylfunktionen, durch die eingeführten unpolaren Reste säulenchromatographisch aufreinigen. Zum anderen wurden durch die Phenylringe Chromophore erhalten, die eine Kontrolle des Reaktionsverlaufs erleichterten. Die Differenzierung der vier Alkoholfunktionen wurde durch Zugabe eines fünffachen Überschusses an Pentaerythrit, beziehungsweise zwanzigfachen Überschusses bezogen auf die Hydroxylfunktionen, erzielt. Die Hydroxylgruppen von 2 wurden im Folgenden mittels Tosylchlorid in Pyridin zu 3 umgesetzt. Nukleophile Substitution mit Natriumazid in Dimethylformamid führte zu Verbindung 4, welche anschließend mittels einer Staudinger-Reduktion in das Triamin 5 umgewandelt wurde (Staudinger \& Meyer (1919)). Die Aminfunktionen wurden bei Raumtemperatur in Ethanol durch Umsetzung mit Di-tert-butyldicarbonat geschützt, um bei der späteren Oxidation der Hydroxylgruppe eine gleichzeitige Oxidation der Amine zum $N$-Oxid zu verhindern. Die Silyletherschutzgruppe an $\mathbf{6}$ erwies sich jedoch im Folgenden als äußerst stabil, da selbst durch mehrtägiges Rühren in $1 \mathrm{M}$ Tetra- $N$-butylammoniumfluoridlösung die Hydroxylfunktion von 7 nicht freigesetzt werden konnte. Dies ließ sich, wie sich im weiteren Verlauf der Arbeit zeigte, auf den sterischen Anspruch der Boc-Gruppen zurückführen (siehe Abbildungen 4.4 und 4.5). 


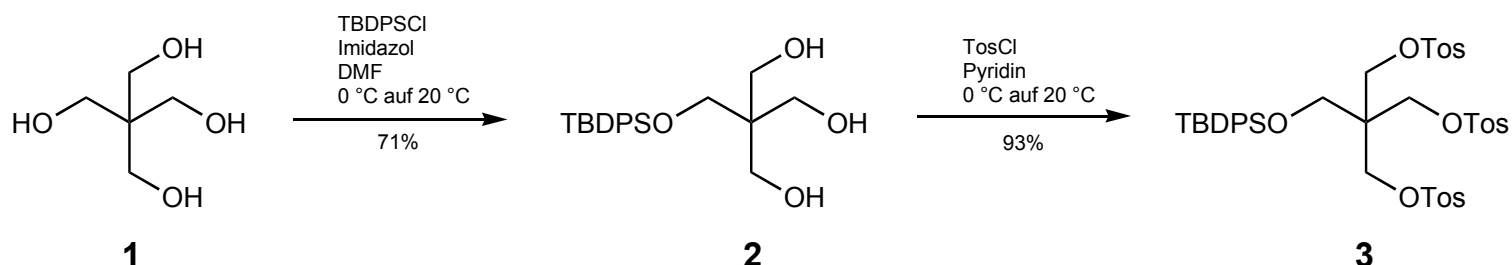

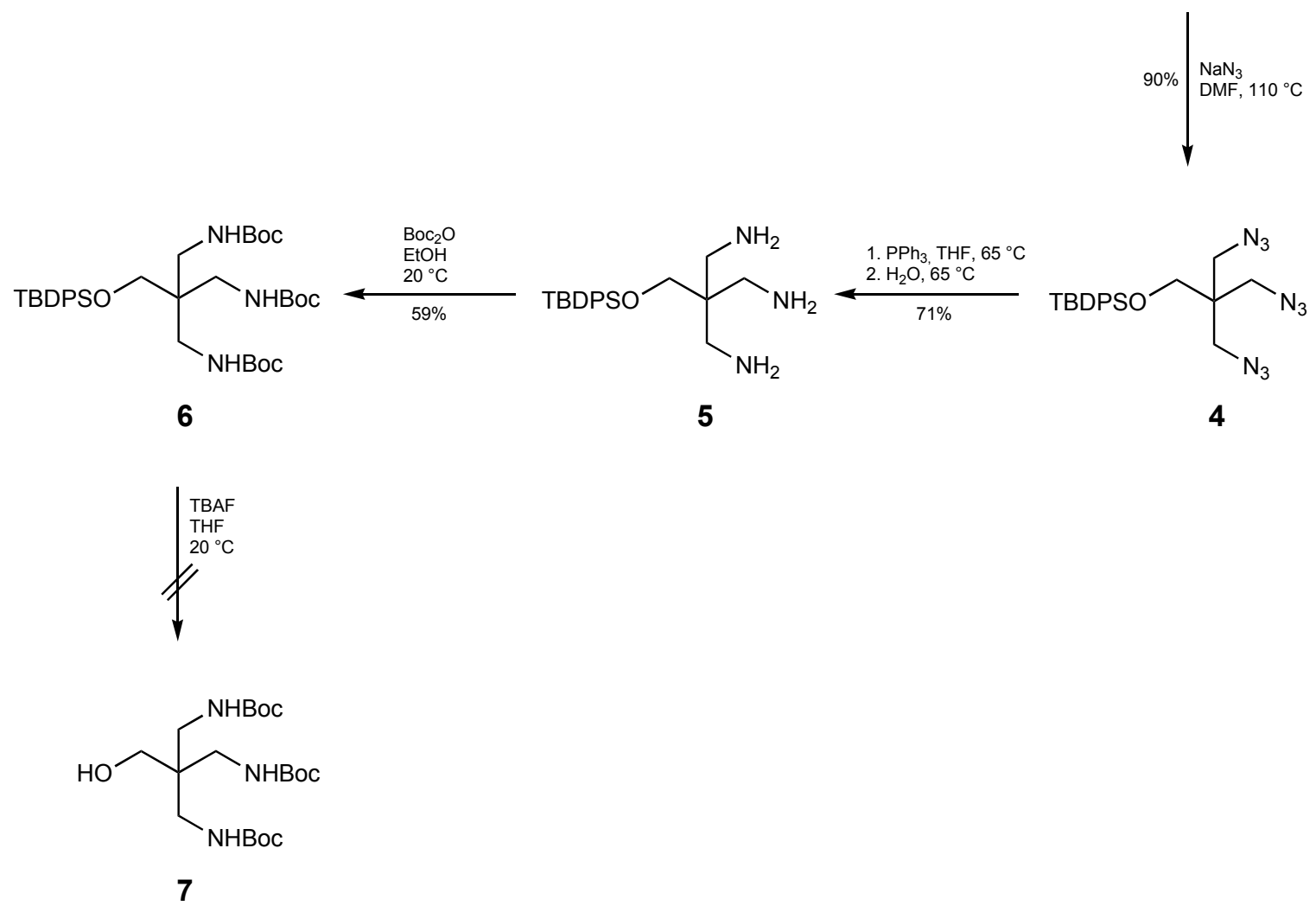

Abb. 4.2: Syntheseansatz ausgehend von Pentaerythrit.

\subsubsection{Weitere Syntheseansätze}

Da sich die Entschützung der Silylschutzgruppe problematisch zeigte, wurde für die weitere Synthese des Tags auf das deutlich teurere Pentaerythritol-tribromid als Edukt zurückgegriffen (siehe Abbildung 4.3). Ein deutlicher Vorteil von 8 liegt darin, dass die Differenzierung der Funktionen bereits vorhanden ist. Es erschien günstig, zunächst den Alkohol zu oxidieren und anschließend das Triamin aufzubauen, da auf diesem Weg die schwierige Oxidation in Gegenwart geschützter Aminfunktionen und die Gefahr der gleichzeitigen $N$-Oxidbildung umgangen werden konnten.

Die Alkoholfunktion wurde, um die Bromatome nicht abzuspalten, möglichst schonend in zwei Stufen zur Carbonsäure oxidiert. Durch Swern-Oxidation (Mancuso 
et al. (1978)) wurde der Aldehyd 9 erhalten. Die Weiteroxidation erfolgte durch Natriumchlorit in einem Gemisch aus Wasser und Acetonitril bei pH 4. Das in der Reaktion entstandene, aggressivere Hypochlorit wurde durch Zugabe von Wasserstoffperoxid abgefangen (Raach \& Reiser (2000); Dalcanale \& Montanari (1986)). Die Carbonsäure 10 wurde im Anschluss in zwei Schritten durch Aktivierung mit Thionylchlorid und Dimethylformamid und Umsetzung mit Benzylalkohol als Benzylester 12 geschützt. Die nachfolgende Substitution der Bromide durch Azide gelang jedoch bei höheren Temperaturen nicht ohne die gleichzeitige Substitution des Benzylesters. Unter milderen Bedingungen (Rye \& Withers (2002)) erfolgte keine Reaktion und auch die Zugabe katalytischer Mengen an Kaliumiodid führte nicht zur Bildung des Triazids 13. Letztendlich wurden keine Bedingungen gefunden, unter denen der nukleophile Angriff ausschließlich am Brom erfolgte, weshalb dieser Reaktionspfad aufgegeben wurde.

Die Reihenfolge der Oxidation und des Aufbaus der Aminfunktionen musste folglich vertauscht werden (siehe Abbildung 4.4). Ausgehend von 8 wurde das $N$-tert-<smiles>OCC(CBr)(CBr)CBr</smiles>
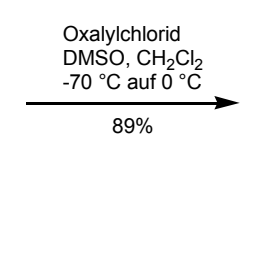

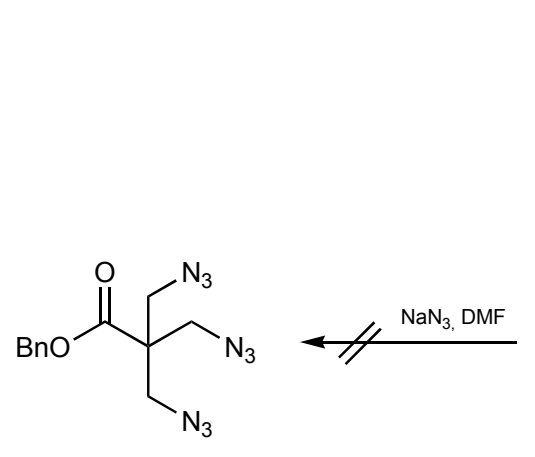

13

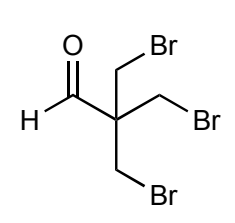

9

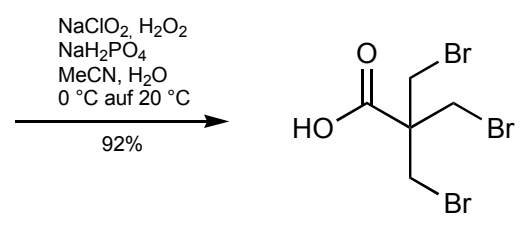

10

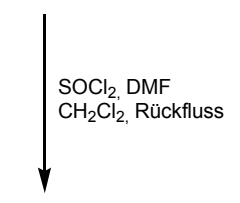

Abb. 4.3: Erster Syntheseansatz ausgehend von Pentaerythritol-tribromid. Zunächst wurde die Hydroxylfunktion in zwei Schritten oxidiert, um anschließend Brom gegen Azid zu substituieren.

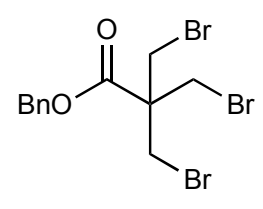

12

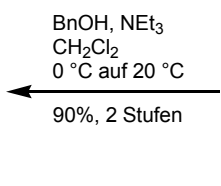

$\mathrm{Cl}$<smiles>O=C(Cl)C(CBr)(CBr)CBr</smiles>

11 
butoxycarbonyl-geschützte Triamin 7 zunächst in einer Eintopfsynthese in $N$-Methylpyrrolidon dargestellt (Saito et al. (1989); Pihko et al. (2001)). Dabei wurde als erstes Bromid gegen Azid substituiert, gefolgt von einer Palladium-vermittelten Hydrogenolyse und anschließender Umsetzung mit Di-tert-butyldicarbonat. Die Ausbeute bei dieser Reaktionsführung war mit 5\% allerdings sehr gering. Durch einen Wechsel des Lösungsmittels konnte die Ausbeute auf über $30 \%$ deutlich verbessert werden. Die nukleophile Substitution wurde dabei in Dimethylformamid durchgeführt. Anschließend wurde das Lösungsmittel für die Hydrogenolyse und Schützung der Amine gegen Ethylacetat ausgetauscht, ohne das potentiell explosive Triazid 14 zu isolieren. Die Möglichkeiten zur Oxidation der Hydroxylgruppe waren durch die Säurelabilität der Boc-Gruppen eingeschränkt. Die Reaktionsführung unter SwernBedingungen brachte keinen Umsatz (Yokoyama et al. (2007)). Ein unter basischen Bedingungen arbeitendes Oxidationsmittel ist das Perruthenation, welches im Bereich von pH 8-12 stabil ist (Bailey et al. (1993)). Die Oxidation zur Carbonsäure 15 erfolgte in zwei Stufen. Im ersten Schritt wurde die Hydroxylgruppe zum Aldehyd oxidiert. Dieser konnte jedoch nicht direkt weiter oxidiert werden. Erst durch die Anlagerung von Wasser zum entsprechenden Aldehydhydrat wurde der zweite Oxidationsschritt möglich, weshalb stets in Gegenwart von Wasser gearbeitet wurde. Perruthenat wurde dabei katalytisch im Bereich von 5-10 mol\% unter Zusatz von Kooxidationsmitteln wie $N$-Methylmorpholin- $N$-oxid (NMO) verwendet (Lenz \& Ley (1997); Ley et al. (1994)). Tetra- $N$-propylammoniumperruthenat (TPAP) ist auf Grund des unpolaren Gegenions in organischen Solventien wie Acetonitril oder Dichlormethan löslich. Die Reaktionen verliefen allerdings auch bei Temperaturen von $50{ }^{\circ} \mathrm{C}$ äußerst langsam (nach 144 Stunden waren jeweils nur 5-15\% der Zielverbindung zu isolieren). Der geringe Umsatz lässt sich an Hand einer 3DStrukurvorhersage von 7 mit dem Programm MacroModel erklären (siehe Abbildung 4.5). Hier ist deutlich zu sehen, dass die Hydroxylfunktion von den drei BocSchutzgruppen teilweise umschlossen und somit stark abgeschirmt wird. Lediglich eine Seite verbleibt zugänglich.

Folglich wurde das sterisch ebenfalls anspruchsvolle Tetra- $N$-propylammoniumkation durch Kalium ersetzt, wodurch allerdings die Löslichkeit im organischen Solvens ver- 


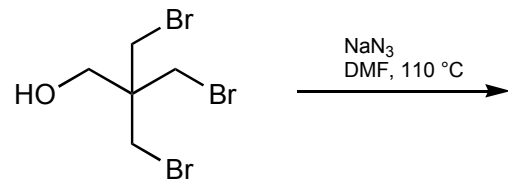

8<smiles>NCC(CN)(CN)C(=O)OCc1ccccc1</smiles>

17

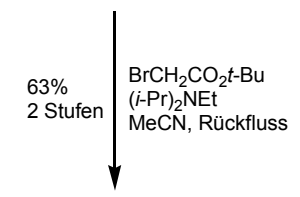

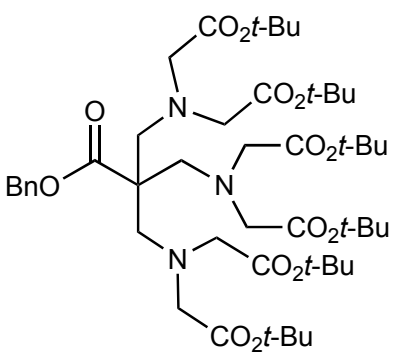

18

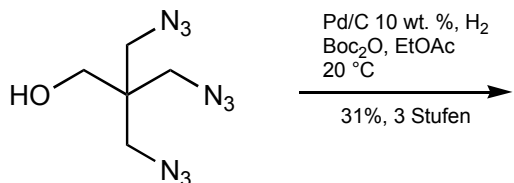

14 $\longleftarrow \begin{aligned} & \mathrm{BnBr}, \mathrm{K}_{2} \mathrm{CO}_{3} \\ & \mathrm{DMF}, 50{ }^{\circ} \mathrm{C}\end{aligned}$

16

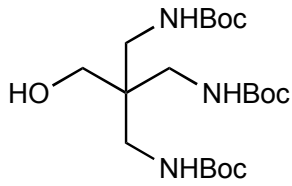

7

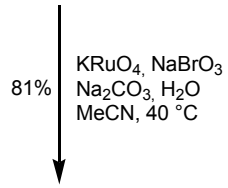

15

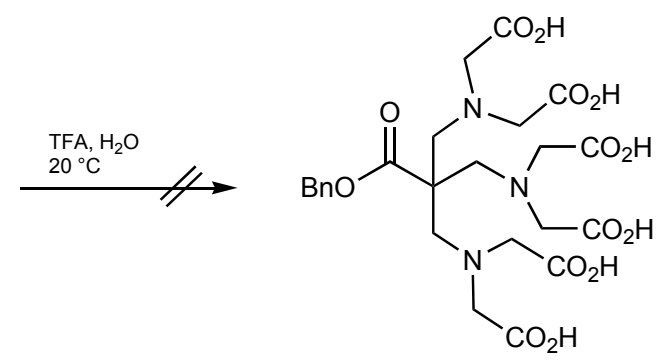

19

Abb. 4.4: Zweiter Syntheseansatz ausgehend von Pentaerythritol-tribromid. Die Reihenfolge von Oxidation und Aufbau der Aminfunktion wurde vertauscht. Bei der Entschützung der Carboxylfunktionen wurde jedoch nicht das Zielmolekül 19 erhalten. 


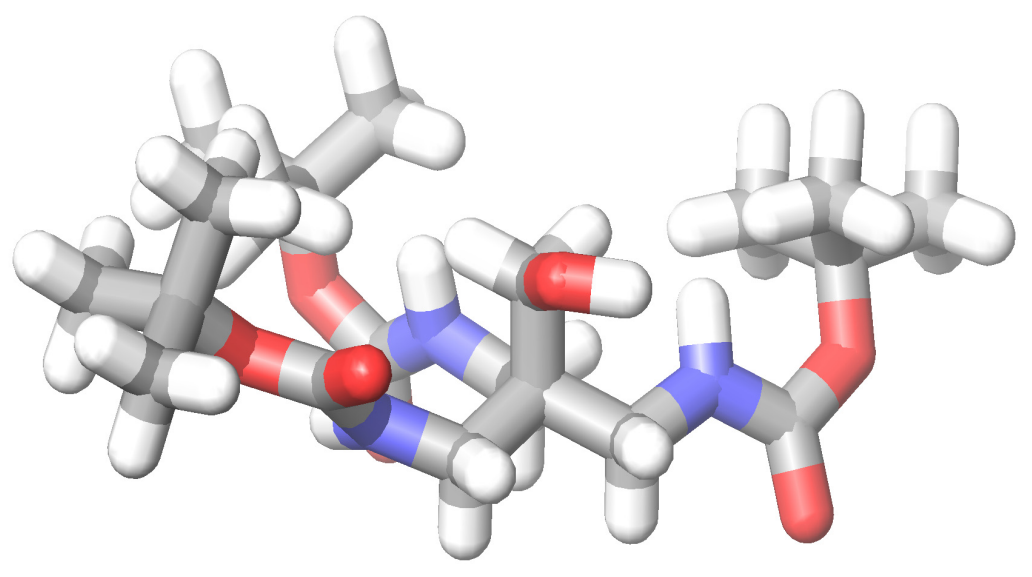

Abb. 4.5: Strukturvorhersage von 7. Die Farben sind wie folgt zugeordnet: Kohlenstoff: grau, Wasserstoff: weiß, Sauerstoff: rot, Stickstoff: blau.

loren ging. Bailey et al. haben Oxidationsreaktionen durch Kaliumperruthenat in einer 1M wässrigen Lösung von Natriumbromat und Natriumcarbonat beschrieben. Mit Acetonitril als organischem Solvens wurde 7 phasentransferkatalytisch oxidiert. Nach 192 Stunden war mittels LC-MS Analyse kein Edukt mehr nachweisbar. Durch Optimierung der Salzkonzentrationen wurde ein homogenes Reaktionsgemisch erhalten. Im Zuge der gleichzeitigen Temperaturerhöhung auf $40{ }^{\circ} \mathrm{C}$ konnte die Reaktionszeit auf unter 20 Stunden reduziert werden. Im Anschluss wurde die freie Säurefunktion als Benzylester 16 geschützt und durch Abspaltung der Boc-Gruppen in Trifluoressigsäure die Aminogruppen von 17 freigesetzt. Diese wurden durch Refluxieren in Acetonitril in Gegenwart einer Base mit Bromessigsäure-tert-butylester zur Verbindung $18 \mathrm{~N}$-alkyliert. Anschließend sollten die sechs Carboxylgruppen des Tags testweise zu Verbindung 19 entschützt werden. Dafür mussten die tertButylester mit Trifluoressigsäure gespalten werden. Dies führte jedoch neben der Esterhydrolyse gleichzeitig zu einer, mit einer Fragmentierung verbundenen Umlagerung, welche vermutlich über den in Abbildung 4.6 vorgeschlagenen Mechanismus abläuft.

Die Protonierung eines Stickstoffs in 20 und folgende Abspaltung eines sekundären Amins führt zu dem primären Carbokation 21, welches sich anschließend durch eine [1,3]-Umlagerung zu dem tertiären Carbokation 22 stabilisiert. Eine weitere Fragmentierung führt zum Acrylat 23, welches bei der Verwendung von 95 \%iger 
Trifluoressigsäure quantitativ erhalten wurde. Die Triebkraft der Reaktion beruht dabei auf der Ausbildung eines Michael-Systems. In der Folge wurde versucht, die tert-Butylester unter milderen Bedingungen zu entschützen. Allerdings war diese Zersetzung auch bei der Reaktion mit unterschiedlich konzentrierter Ameisensäure, einer deutlich schwächeren Säure im Vergleich zu Trifluoressigsäure, zu beobachten $\left(\mathrm{pK}_{a}(\right.$ Ameisensäure $)=3.75, \mathrm{pK}_{a}($ Trifluoressigsäure $\left.)=0.77\right)$. Nach 1.5 Stunden Reaktionszeit in 85 \%iger Ameisensäure waren bei der Reaktionskontrolle mittels ESI-MS neben der Masse von 23 sowohl Signale unvollständig entschützter Zwischenstufen, als auch die Masse von 24 nachweisbar. Dies indiziert, dass die Umlagerung mit einer ähnlichen Geschwindigkeit wie die Entschützung abläuft.

Aufgrund der Säurelabilität des Molekülgerüsts wurde die Schutzgruppenstrategie verändert und der Chelator als Trimethylsilylethanolester (TMSE-Ester) geschützt (Sieber (1977)). Dies ist in Abbildung 4.7 dargestellt. Die Carboxylfunktionen können

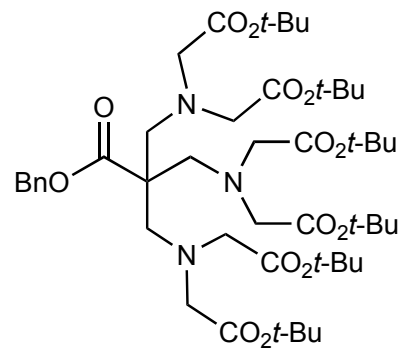

18

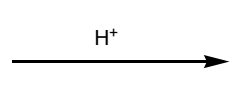<smiles>[R]OC(=O)CN(CC(=O)O)CC(=O)OCc1ccccc1</smiles>

23: $\mathrm{R}^{\prime}=\mathrm{H}$

24: $\mathrm{R}^{\prime}=t-\mathrm{Bu}$<smiles>[R]OC(=O)CN(CC(=O)O)CC(CN(CC(=O)O)CC(=O)O)(CN(CC(=O)O)CC([R])[R])C(=O)OCc1ccccc1</smiles>

20

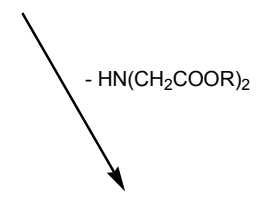

Abb. 4.6: Mechanismus der Fragmentierung von 18 zum Acrylat. Dabei steht $R$ in den Strukturen 20-22 für $\mathrm{H}$ oder $t$-Bu, R' für $\mathrm{H}$ (23) bzw. für $t$-Bu (24). 
hierbei durch Fluoridionen unter Abspaltung von Ethen freigesetzt werden. Hierzu wurde zunächst das neue Alkylierungsmittel 26 aus Bromessigsäurebromid 25 synthetisiert und anschließend mit 17 zu 27 umgesetzt. Die Entschützung der TMSEEster erfolgte in 1M TBAF-Lösung in Tetrahydrofuran. Zwar konnte das Zielmolekül 19 in Spuren isoliert werden, jedoch war 23 erneut das Hauptprodukt der Reaktion. Dass diese Fragmentierung selbst in schwach acidem Medium abläuft, verdeutlichte erneut die enorme Triebkraft der Acrylatbildung. Diese hohe Säureempfindlichkeit in Kombination mit der geringen Ausbeute an 19 machte an dieser Stelle einen komplett neuen Ansatz notwendig. Um die Ausbildung des Michael-Systems zu unterbinden, musste zwischen die Carbonylfunktion und das quatäre Kohlenstoffatom ein Abstandshalter eingefügt werden. Um zusätzliche Rotationsfreiheitsgrade zu vermeiden, musste dieses Verbindungstück axialsymmetrisch sein. Die Wahl fiel dabei auf einen 1,4-disubstituierten Phenylring, da dieser chemisch leichter zu modifizieren ist, als beispielsweise ein Ethinderivat. Zwar wäre, bei analoger Fragmentierungsreakti-

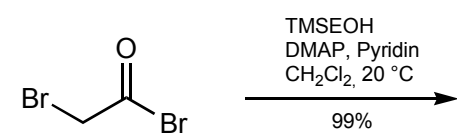

25<smiles>CCCCOC(=O)CBr</smiles>

26<smiles>CC(C)COC(=O)C(CN)(CN)CN</smiles>

17

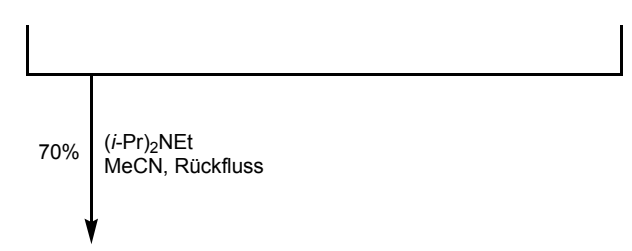<smiles>O=C(O)CN(CC(=O)O)CC(CN(CC(=O)O)CC(=O)O)(CN(CC(=O)O)CC(=O)O)C(=O)OCc1ccccc1</smiles>

19

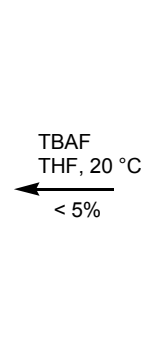

${ }^{20}{ }^{\circ} \mathrm{C} \mathrm{BnO}$

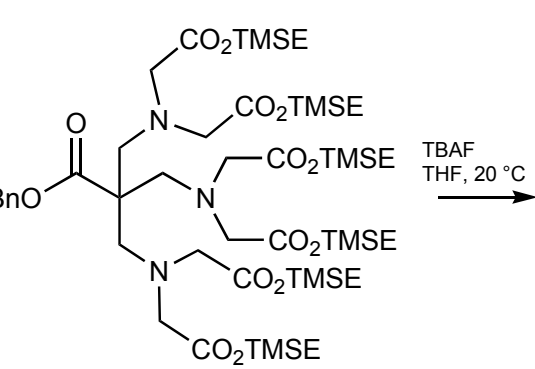

27<smiles>C=C(CN(CC(=O)O)CC(=O)O)C(=O)OCc1ccccc1</smiles>

23

Abb. 4.7: Dritter Syntheseansatz ausgehend von Pentaerythritol-tribromid. Die Carboxylfunktionen wurden als fluoridlabile TMSE-Ester geschützt. 
on, auch hier die Ausbildung eines konjugierten Systems in Form eines Styrolderivats möglich, allerdings sollte die Triebkraft deutlich geringer sein (siehe Abbildung 4.8).

\subsection{Synthese von Cys-Ph-TAHA}

Die finale Synthese des Cys-Ph-TAHA-Tags (Cysteinyl-Phenyl-TAHA) ist in Abbildung 4.9 gezeigt. Ausgehend von 2-(4-Bromphenyl)ethanol 28 wurde die Hydroxylfunktion zunächst mit dem Dess-Martin Periodinan in Dichlormethan zum entsprechenden Aldehyd 29 oxidiert (Dess \& Martin (1983)). Im folgenden Reaktionsschritt wurde die $C_{3}$-Achse aufgebaut. Paraformaldehyd diente hierbei einerseits als $\mathrm{C}_{1}$-Einheit, andererseits als Reduktionsmittel (Hellmann et al. (1995)). Trotz der drei Hydroxylfunktionen ließ sich 30 säulenchromatographisch leicht in Form eines farblosen Öls isolieren. Die anschließende Umwandlung der Alkohol- in Aminfunktionen erfolgte analog zu den zuvor beschriebenen Reaktionen. Die Aktivierung der Hydroxylgruppen mit Tosylchlorid ergab 31, nukleophile Substitution mit Natriumazid in Dimethylformamid führte zu 32. An dieser Stelle wurde das dreifache Azid auf Grund der potentiellen Explosivität organischer Azide nicht isoliert, sondern stets in Lösung behalten. Das Lösungsmittel wurde gewechselt, indem die Reaktionslösung der Azidsubstitution auf Wasser gegossen wurde, 32 daraus mit Dichlormethan extrahiert und anschließend, unter Ausnutzung der unterschiedlichen Siedepunkte, in eine Tetrahydrofuranlösung überführt wurde. Die Reduktion<smiles>CS(=O)(=O)SC[C@H](NC(=O)c1ccc(C(CN(C[AsH2]C2CC2)CC(=O)O)(CN(CC(=O)O)CC(=O)O)CN(CC(=O)O)CC(=O)O)cc1)C(=O)O</smiles>

Abb. 4.8: Neues Zielmolekül Cys-Ph-TAHA: Zwischen die $C_{3}$-Achse und die Carbonylfunktion, an der der Linker angebracht ist, wurde als Abstandshalter ein Phenylring eingefügt. 


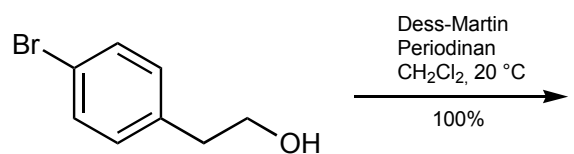

28

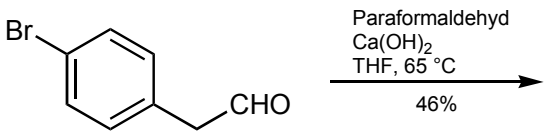

29<smiles>OCC(CO)(CO)c1ccc(Br)cc1</smiles><smiles>[3H][C@H](C)C(C)C</smiles>

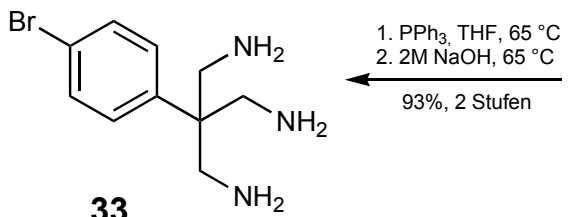<smiles>NCC(CN)(CN)c1ccc(Br)cc1</smiles>

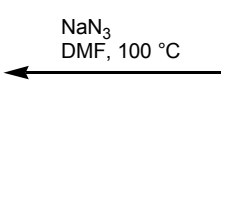<smiles></smiles>

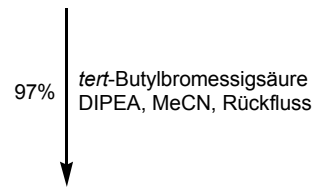<smiles>COC(=O)CN(CC(C)(C)C)CC(CN(CCN(CC(=O)OC(C)(C)C)CC(C)(C)C)CC(=O)OCC(C)(C)C)(CN(CC(=O)OC)CC(C)(C)C)c1ccc(Br)cc1</smiles>

34

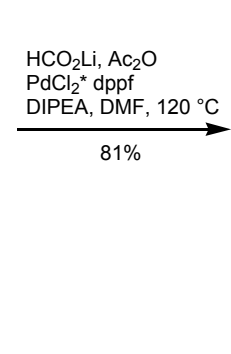

$\mathrm{PdCl}_{2}^{*} \mathrm{dppf}$

DIPEA, DMF, $120^{\circ} \mathrm{C}$<smiles>COC(=O)CN(CC(=O)OC)CC(CN(CCN(CC(=O)OC(C)(C)C)CC(C)(C)C)CC(=O)OC(C)(C)C)(CN(CC(=O)OC)CC(C)(C)C)c1ccc(C(=O)O)cc1</smiles>

35

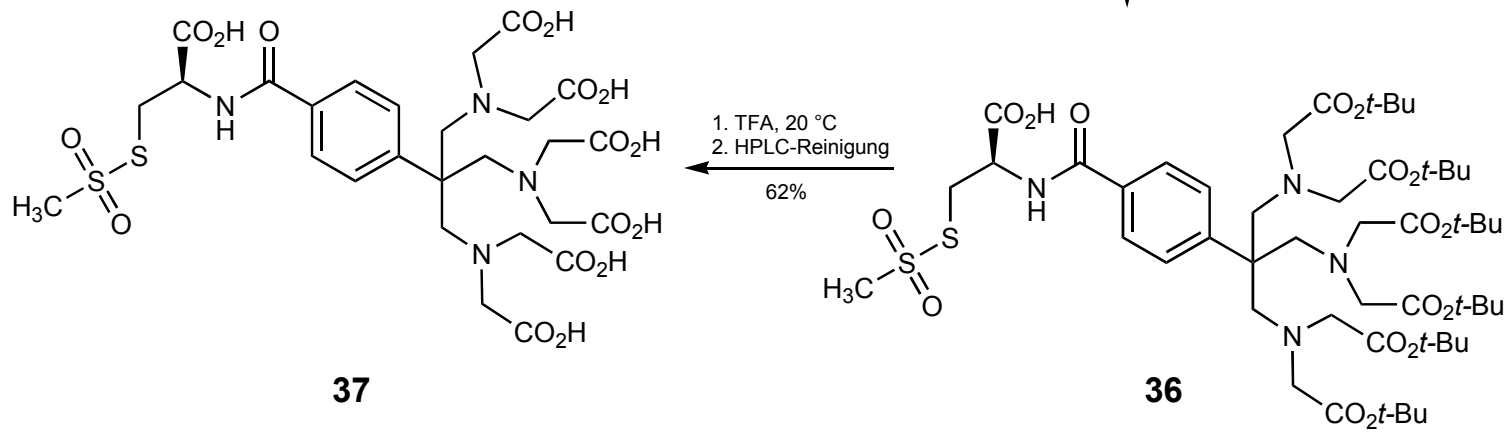

Abb. 4.9: Syntheseschema von Cys-Ph-TAHA. 


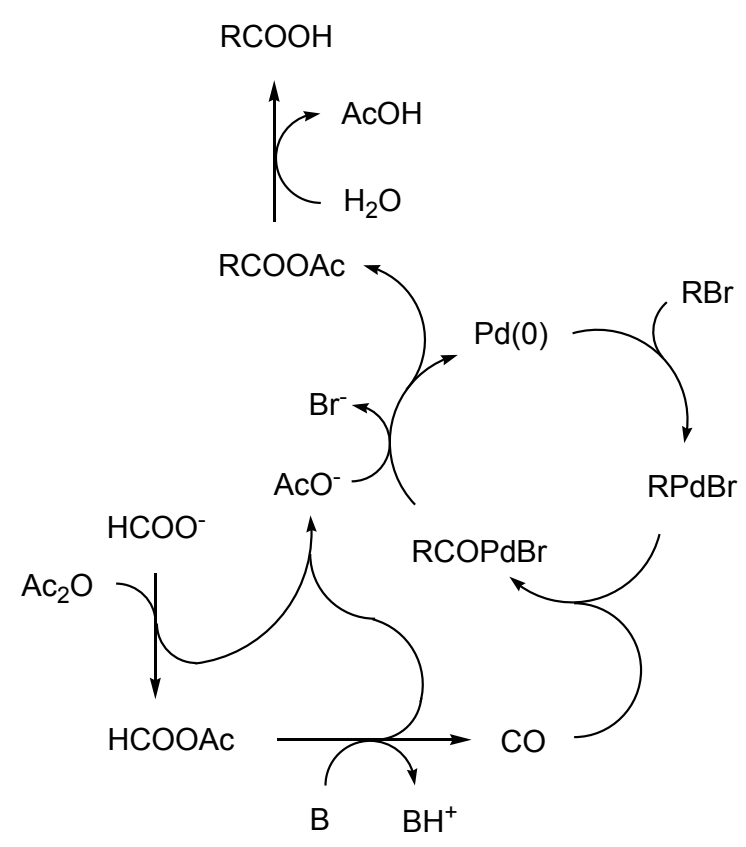

Abb. 4.10: Reaktionsschema der Hydroxycarbonylierung. Kohlenmonoxid wird in situ aus Lithiumformiat und Essigsäureanhydrid gebildet und insertiert anschließend in die Kohlenstoff-Palladiumbindung. Durch reduktive Eliminierung und Hydrolyse wird schließlich die Carbonsäure freigesetzt.

der Azide erfolgte unter Staudingerbedingungen, da eine Hydrogenolyse zum Verlust des Broms am Phenylring geführt hätte (Bunnage et al. (2003); Staudinger \& Meyer (1919)). Im nächsten Schritt wurde 33 mit Bromessigsäure-tert-butylester $N$-alkyliert. Der Reaktionsverlauf wurde dabei per HPLC kontrolliert. Nachdem der Chelator synthetisiert war, musste noch das Verbindungsstück aufgebaut werden, über das der Tag mit dem Protein verbunden werden kann. Hierzu wurde das arylische Brom von 34 mittels einer Palladium-katalysierten Hydroxycarbonylierung in eine Carbonsäure überführt. Als Ligand wurde 1,1'-Bis(diphenylphosphino)ferrocen, kurz dppf, verwendet. Bei der Reaktion bildet sich zunächst ein gemischtes Anhydrid aus dem Formiation und Essigsäureanhydrid. Basenvermittelt, sowie durch die erhöhte Temperatur, zerfällt dieses und setzt in situ Kohlenmonoxid frei, welches in die Bindung zwischen Phenylring und Palladium insertiert. Der Reaktionsmechanismus ist in Abbildung 4.10 schematisch dargestellt (Berger et al. (2006); Cacchi et al. (2003); Kubota et al. (1994)). Die erhaltene Carbonsäure 35 wurde mit S-MesylL-cystein (Weidner \& Block (1972); Hart (1985)) durch Aktivierung mit HATU in 


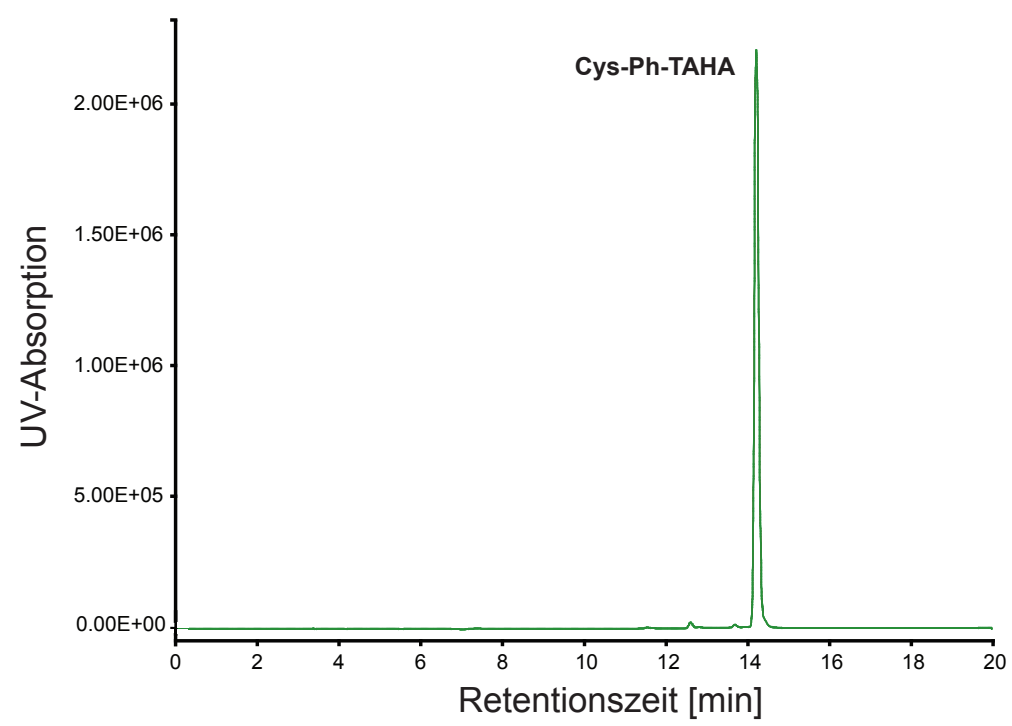

Abb. 4.11: Chromatogramm von HPLC-gereinigtem Cys-Ph-TAHA. Der Peak hat eine Reinheit von > $97 \%$ bei einer Retentionszeit von 14.2 min. Gradient: 0-30\% in $15 \min \left(\mathrm{H}_{2} \mathrm{O} \rightarrow \mathrm{MeCN}\right.$ mit jeweils $0.1 \%$ TFA $)$.

Gegenwart von Hünig-Base gekuppelt. $S$-Mesyl-L-cystein wurde bereits bei früheren Tags als Verbindungsstück verwendet (Leonov et al. (2005); Haberz et al. (2006)). Somit sollte gewährleistet werden, dass sich Unterschiede im Alignmenttensor auf die unterschiedlichen Chelatoren zurückführen lassen. 36 wurde bei Raumtemperatur in konzentrierter Trifluoressigsäure entschützt und Cys-Ph-TAHA 37 erhalten. Der Tag war unter diesen harschen Bedingungen vollständig stabil und zeigte, wie erwartet, keine Fragmentierung, da durch die Einführung des Phenylrings als Abstandshalter zur Carbonylfunktion die Triebkraft der Umlagerung entscheidend herabgesetzt wurde. Abschließend wurde Cys-Ph-TAHA per HPLC gereinigt und lyophylisiert. Das Chromatogramm ist in Abbildung 4.11 gezeigt.

\subsection{Herstellung der NMR-Proben}

Für die Taggingreaktion muss das Protein im reduzierten Zustand, also mit freier Thiolfunktion, vorliegen. Nach der Aufreinigung wurden den Ubiquitinproben TCEP zur Stabilisierung zugesetzt und diese anschließend gefriergetrocknet. Dieses nicht 
flüchtige Reduktionsmittel verhindert die Ausbildung von Disulfidbrücken und muss vor der Taggingreaktion entfernt werden, damit die Bindung von Protein und Tag nicht sofort wieder gebrochen wird. Im Falle von Ubiquitin kann TCEP einfach durch Dialyse entfernt werden, da die Dimerisierung relativ langsam verläuft. Vier Stunden nach Beendigung der Dialyse war mittels Massenspektrometrie nur ein geringes Dimersignal zu sehen, die vollständige Dimerisierung der Probe war erst nach Tagen zu beobachten. Die Reaktion wurde in MOPS Puffer $(50 \mathrm{mM}$, Natriumchlorid $50 \mathrm{mM}$, pH 6.8) bei Raumtemperatur durchgeführt. Die Reaktionskontrolle erfolgte mittels Massenspektrometrie. Dazu wurde ein kleiner Teil der getaggten Proteinlösung gegen den flüchtigen Ammoniumacetatpuffer dialysiert. Auch wenn auf diese Weise keine absoluten Aussagen über die Reaktionsausbeute getroffen werden konnten, war zumindest direkt ersichtlich, ob noch ungetaggtes Protein vorliegt. In diesem Fall wurde die Taggingreaktion wiederholt. Insgesamt waren drei Äquivalente an Tag ausreichend, um das Protein quantitativ zu markieren. Anschließend wurde der Chelator für die Messungen der RDCs und PCSs mit Lanthanoidionen beladen. Zunächst wurde eine Probe der T12C-Mutante vorsichtig mit Terbiumchloridlösung titriert. Im ${ }^{1} \mathrm{H},{ }^{15} \mathrm{~N}$-HSQC-Spektrum zeigten sich Pseudokontaktverschiebungen, die isotropen Peaks nahmen allerdings ab einem Verhältnis von 1:1 zum verschobenen Peak mit zunehmender Konzentration an Terbium nicht weiter ab. Auch die Reaktion mit einem zwanzigfachen Überschuss an Terbiumchlorid mit anschließender Dialyse gegen den Messpuffer lieferte eine Probe mit einer Beladung von nur etwa 50\%. Die Dialyse selbst konnte als Ursache ausgeschlossen werden, da die Beladung durch mehrtägiges Dialysieren nicht weiter abnahm. Um das Problem der unvollständigen, nachträglichen Beladung zu umgehen, wurde Cys-Ph-TAHA im Folgenden vor der Taggingreaktion beladen. Hierzu wurde der Tag mit einem zweifachen Überschuss an Lanthanoidsalz in reinem Wasser für zwei Stunden inkubiert. Durch die Carboxylfunktionen des Tags und das Metallion hat die Lösung etwa $\mathrm{pH}$ 4, was die Beladung des Chelators begünstigt. Das Protein wurde parallel gegen MOPS-Puffer mit pH 8.0 dialysiert. Dies hatte zwei Gründe. Zum einen wird die Reaktivität des Cysteins erhöht, zum anderen fällt der Überschuss an Lanthanoidionen instantan aus, sobald die Proteinlösung zu der ungepufferten, vorbeladenen Taglösung pipettiert wird. 


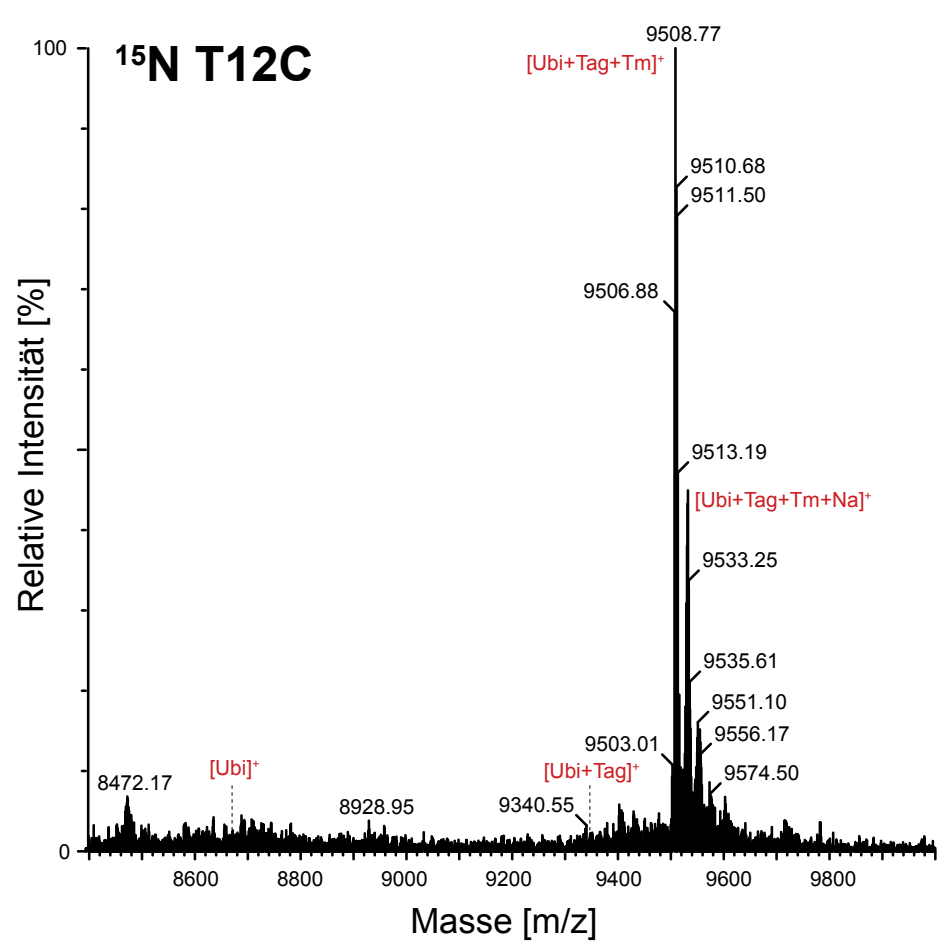

Abb. 4.12: Massenspektrum von ${ }^{15} \mathrm{~N}$-markiertem, Cys-Ph-TAHA-getaggtem T12C, beladen mit $\mathrm{Tm}^{3+}$. Die Massen des ungetaggten, beziehungsweise unbeladenen Proteins sind mit gestrichelten Linien markiert.

Im Anschluss an die Taggingreaktion wurde der überschüssige Metall-bindende Tag durch mehrfaches Waschen mit dem Messpuffer in einem Zentrifugationskonzentrator entfernt. Ein allgemeines Taggingprotokoll ist im experimentellen Teil unter 6.5 gegeben. Im Gegensatz zum unbeladenen Tag waren für einen vollständigen Umsatz fünf Äquivalente des beladenen Tags von Nöten. Zur Bestimmung der Beladung wurden die Peakintensitäten verschiedener Aminosäurereste, deren anisotrope und isotrope Signale im HSQC-Spektrum hinreichend aufgelöst waren, miteinander verglichen (siehe Anhang 7.6). Mit der Methode der Vorbeladung wurden Beladungen von $98 \%$ für T12C erreicht. In Abbildung 4.12 ist ein Massenspektrum von ${ }^{15} \mathrm{~N}$ markiertem T12C, beladen mit Thulium, gezeigt. Es ist kein signifikanter Peak des ungetaggten Proteins zu sehen. Inwieweit das Signal des getaggten aber unbeladenen Ubiquitins zu sehen ist, kann quantitativ nicht als Merkmal der Beladung herangezogen werden, da die Metallionen häufig durch die Ionisierung verloren gehen. In Proben der S57C Mutante betrug der Anteil der anisotropen Komponente 


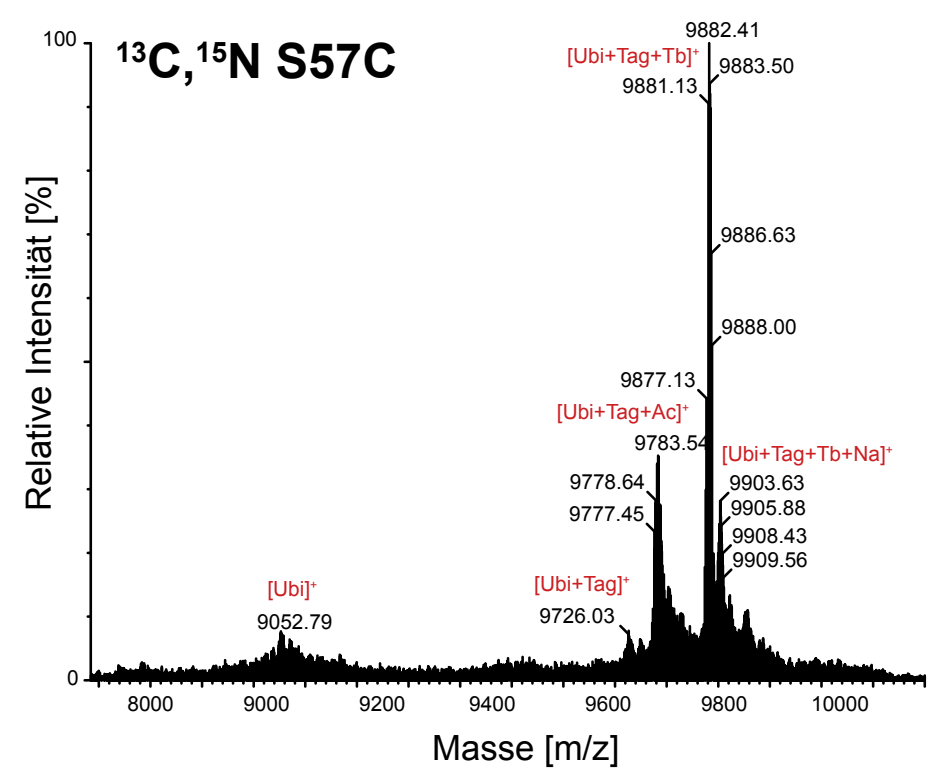

Abb. 4.13: Massenspektrum von ${ }^{13} \mathrm{C},{ }^{15} \mathrm{~N}$-markiertem, Cys-Ph-TAHA-getaggtem S57C, beladen mit $\mathrm{Tb}^{3+}$.

stets etwa 90\% und war damit geringer als für T12C. Wie das beispielhafte Massenspektrum von ${ }^{13} \mathrm{C},{ }^{15} \mathrm{~N}$-markiertem S57C, beladen mit Terbium, in Abbildung 4.13 zeigt, ist die Ursache hierfür eine unvollständige Taggingreaktion. Aber auch durch erneuten Umsatz oder Reaktion mit mehr Äquivalenten an vorbeladenem Cys-PhTAHA wurden die Ausbeuten nicht weiter gesteigert, sondern vielmehr die zuvor beschriebenen 90\% reproduziert. Eine mögliche Erklärung war die Dimerisierung des Proteins. Mittels Massenspektrometrie konnten allerdings keine S57C Dimere nachgewiesen werden. Eine Fragmentierung im Zuge der Ionisierung schied ebenso aus, da Ubiquitindimere bei den angelegten Ionisierungsenergien nicht auseinander brechen. Warum letztendlich etwa 10\% an S57C inaktiviert, beziehungsweise für den Tag unzugänglich waren, konnte nicht bestimmt werden.

Insgesamt wurde durch die Vorbeladung des Cys-Ph-TAHA Tags und die anschließende Reaktion eine effektive und reproduzierbare Methode gefunden, Ubiquitin in hoher Ausbeute paramagnetisch zu markieren. Durch sorgfältiges Entfernen der überschüssigen Lanthanoidionen aus den Proben wurden Spektren von hoher Qualität erhalten. In Abbildung 4.14 sind beispielhaft ${ }^{1} \mathrm{H}-\mathrm{Spektren}$ von Cys-Ph-TAHAgetaggtem T12C gezeigt. Es ist zu sehen, dass die Linien durch die Beladung mit 

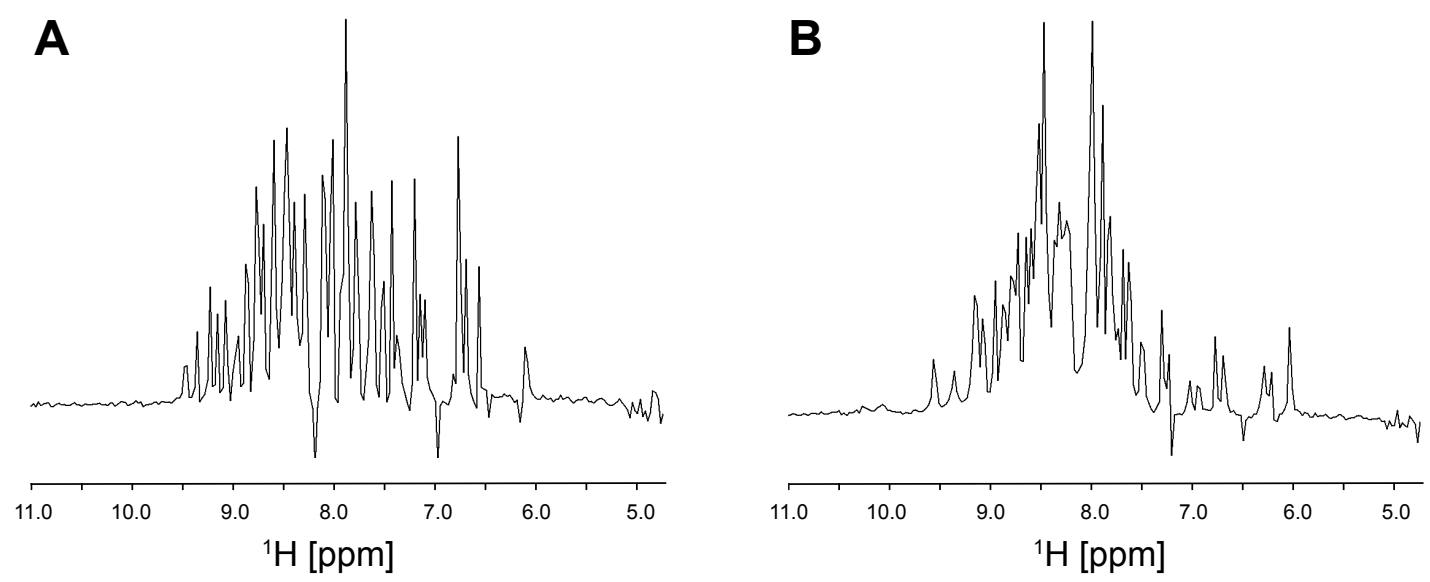

Abb. 4.14: Ausschnitte aus den ${ }^{1} \mathrm{H}-$ Spektren der Amidregion von Cys-Ph-TAHA getaggtem Ubiquitin. Die Proben wurden mit diamagnetischem $\mathrm{Lu}^{3+}$ (A) bzw. paramagnetischem $\mathrm{Tm}^{3+}(\mathrm{B})$ beladen und unter identischen Bedingungen vermessen.

Thulium im Vergleich zu diamagnetischem Lutetium nur wenig verbreitert waren. Beide Spektren wurden mit den gleichen Parametern aufgenommen.

Innerhalb dieser Arbeit wurden verschiedene Proben hergestellt. Für die letztendliche Datenauswertung wurden acht Proben mit einer jeweiligen Konzentration von etwa $1.5 \mathrm{mM}$ verwendet. Dabei wurden die beiden zur Verfügung stehenden Ubiquitinmutanten (T12C und S57C) jeweils mit $\mathrm{Tb}^{3+}, \mathrm{Tm}^{3+}$ und $\mathrm{Lu}^{3+}$ vorbeladenem Cys-Ph-TAHA getaggt. Im Folgenden werden diese Proben durch die Mutante in Kombination mit dem verwendeten Metall angegeben (beispielsweise T12C Tm). Zusätzlich wurde für beide Mutanten eine getaggte aber unbeladene Probe hergestellt. Diese wurden als isotrope Referenzproben für die RDC-Messungen verwendet.

Für die PCS-Messungen wurden als Referenz T12C Lu und S57C Lu vermessen, da das diamagnetische $\mathrm{Lu}^{3+}$ im Vergleich zu den Lanthanoidionen eine ähnliche Ionengröße und Elektronenkonfiguration aufweist. In Abbildung 4.15 ist ein Ausschnitt aus den überlagerten ${ }^{1} \mathrm{H},{ }^{15} \mathrm{~N}-\mathrm{HSQC}-$ Spektren von S57C Lu und ungetaggtem S57C gezeigt. Einige Aminosäurereste, wie E18 oder D58, die sich in der Struktur nahe am Tag befinden, wurden stark beeinflusst durch die Anwesenheit des Tag-gebundenen Metalls. Die unterschiedlichen chemischen Verschiebungen beruhen aber vor allem 


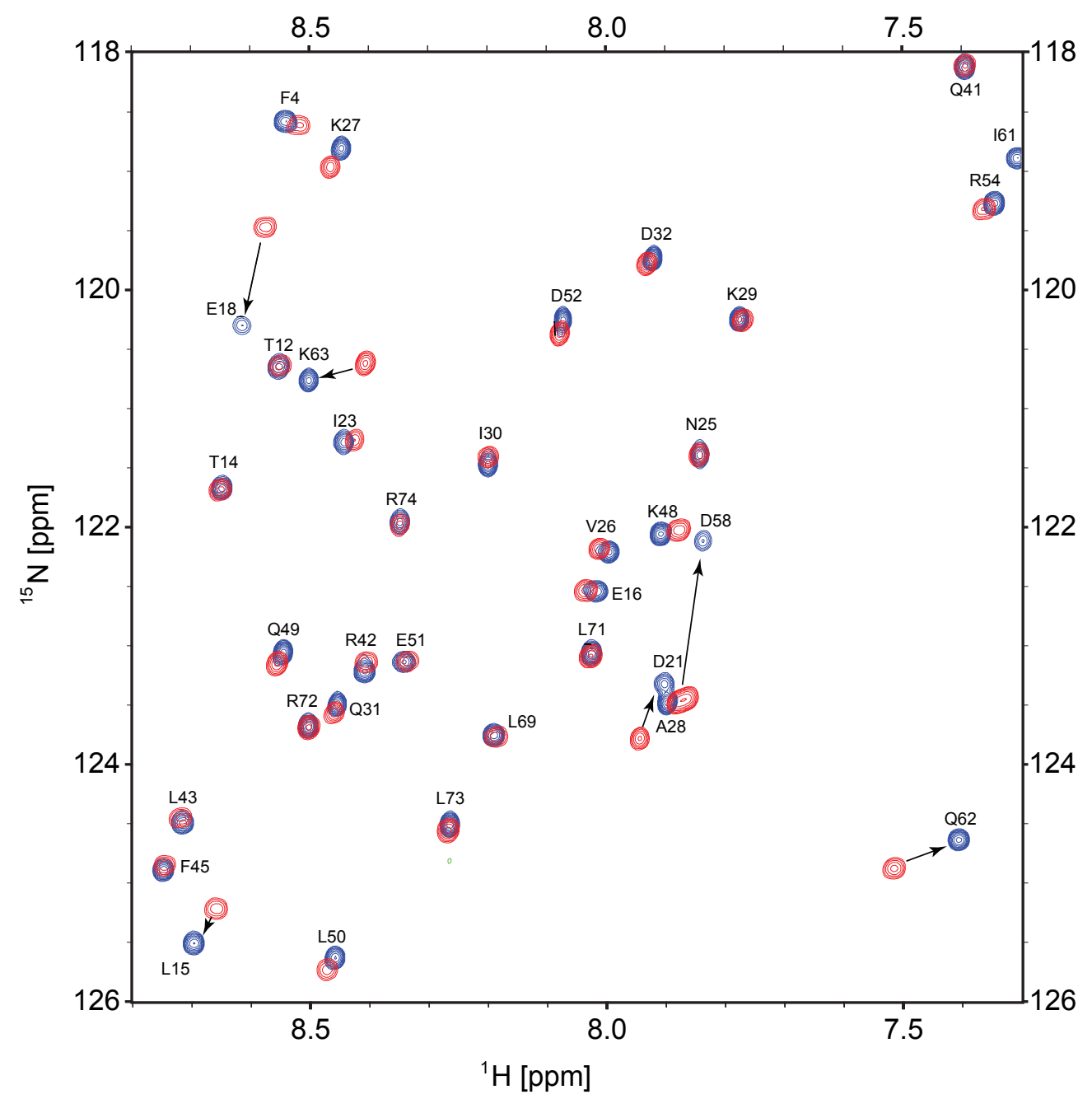

Abb. 4.15: Überlagerung der ${ }^{1} \mathrm{H},{ }^{15} \mathrm{~N}-\mathrm{HSQC}$ Spektren von ungetaggtem Ubiquitin S57C (rot) und Cys-Ph-TAHA getaggtem Ubiquitin-S57C, beladen mit $\mathrm{Lu}^{3+}$ (blau).

auf Ladungsveränderungen rund um den Tag. Die Einflüsse des Tags auf die Struktur sind hingegen gering, wie die im folgenden Kapitel vorgestellten Ergebnisse belegen. 


\section{Kapitel 5}

\section{Ergebnisse und Diskussion}

\subsection{Pseudokontaktverschiebungen}

Als Pseudokontaktverschiebung (PCS) wird die Änderung der chemischen Verschiebung eines Kerns im Vergleich zum isotropen Spektrum bezeichnet, die durch die abstandsabhängige Interaktion mit einem magnetisch anisotropen Metallion entsteht (siehe Kapitel 2.2). Um die PCSs des Proteinrückgrats zu bestimmen, wurden ${ }^{1} \mathrm{H},{ }^{15} \mathrm{~N}-\mathrm{HSQC}-S p e k t r e n$ einer anisotropen und einer isotropen Referenzprobe aufgenommen.

In den Abbildungen 5.1 und 5.2 sind die HSQC-Spektren von T12C Tb und T12C Tm, mit jeweils T12C Lu überlagert, gezeigt. Aus Gründen der Übersicht wurden negative Signale, die durch Faltung während der Acquisition entstanden sind, ausgeblendet. Im Anhang in den Tabellen 7.2 und 7.3 sind die vollständigen chemischen Verschiebungen und die resultierenden PCSs angegeben. Die erhaltenen Pseudokontaktverschiebungen sind mit durchgezogenen Linien eingezeichnet. Für T12C Tm konnten insgesamt 65 PCSs extrahiert werden. Somit wurden im Vergleich zur isotropen Probe lediglich fünf Signale durch die paramagnetische Relaxationsverstärkung verloren. Mit T12C Tb wurden 59 PCSs erhalten. Im HSQC-Spektrum waren die gleichen Aminosäurereste, wie für T12C Tm nicht sichtbar. Darüber hinaus fehlten weitere Signale in der unmittelbaren Umgebung dieser Reste, beziehungsweise waren nur noch schwach nachweisbar (T7, G10, I13 und F45). Dies deutet bereits auf eine sehr ähnliche Metallposition in beiden Proben hin. Der zusätzliche Signalverlust 
im Vergleich von Terbium zu Thulium lässt sich durch die stärkeren PRE-Effekte von Terbiumionen erklären (Pintacuda et al. (2007)). In Abbildung 5.3 sind diese Aminosäurereste in der Ubiquitinstruktur (PDB-Kode: 1D3Z) farbig markiert. Erwartungsgemäß befinden sie sich auf derselben Seite des Proteins. Reste, die auch im isotropen Spektrum keinen Peak zeigten, wurden nicht mit berücksichtigt.

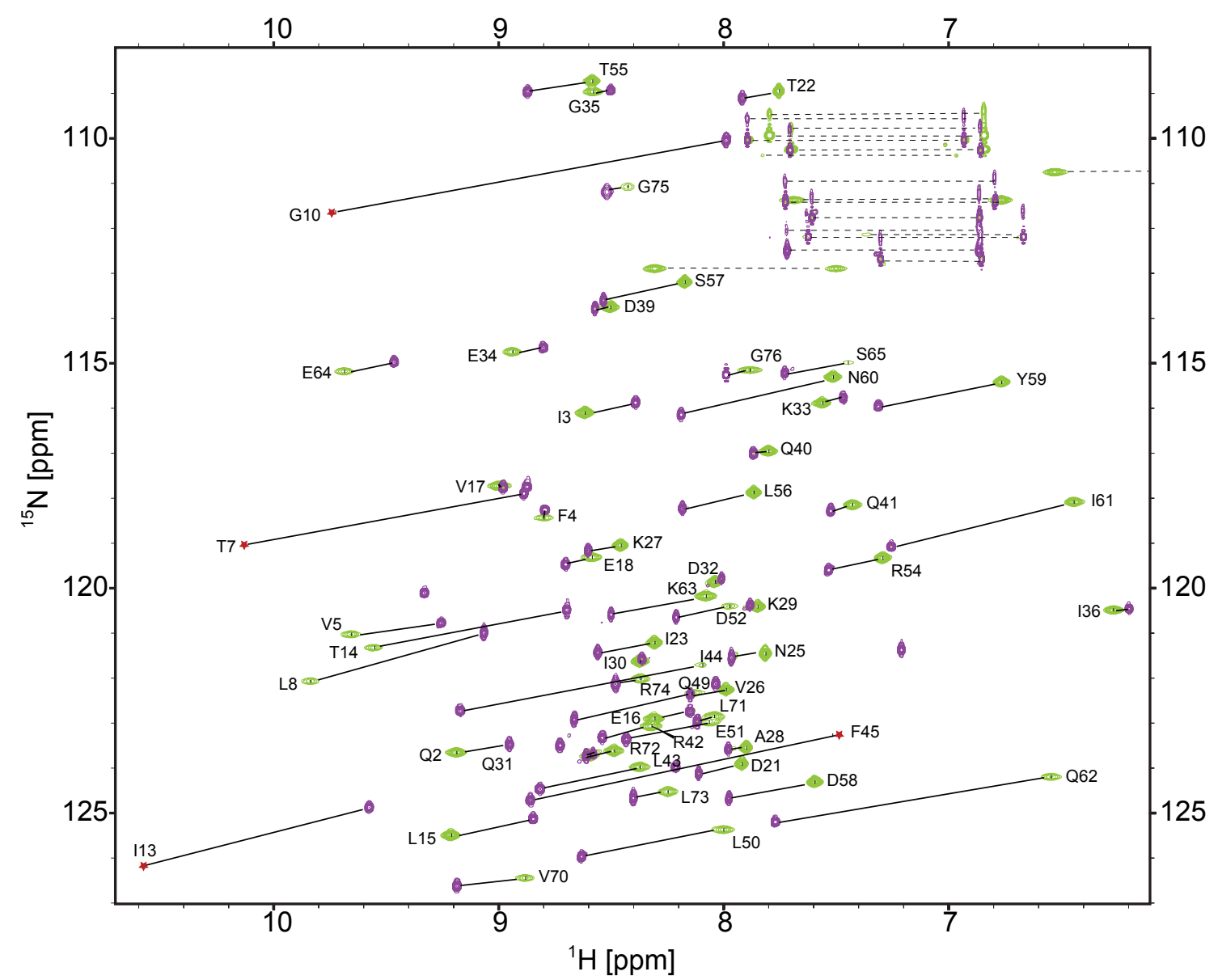

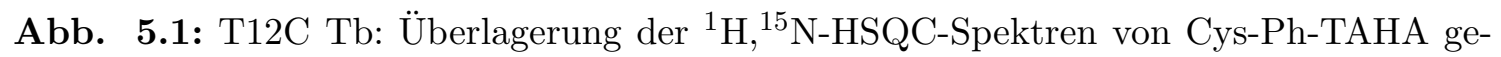
taggtem Ubiquitin-T12C, beladen mit $\mathrm{Tb}^{3+}$ (grün) und $\mathrm{Lu}^{3+}$ (violett). PCSs sind mit durchgezogenen Linien eingezeichnet, Signale von Seitenketten sind durch unterbrochene Linien angezeigt. Die gefalteten Signale (States-TPPI) der Aminosäurereste T9 und S20 sind ausgeblendet. Die Peaks von T7, G10, I13 und F45 sind schwach und mit einem Stern (rot) markiert. Eine vollständige Liste der chemischen Verschiebungen der insgesamt 59 PCSs ist im Anhang in Tabelle 7.2 gegeben. 


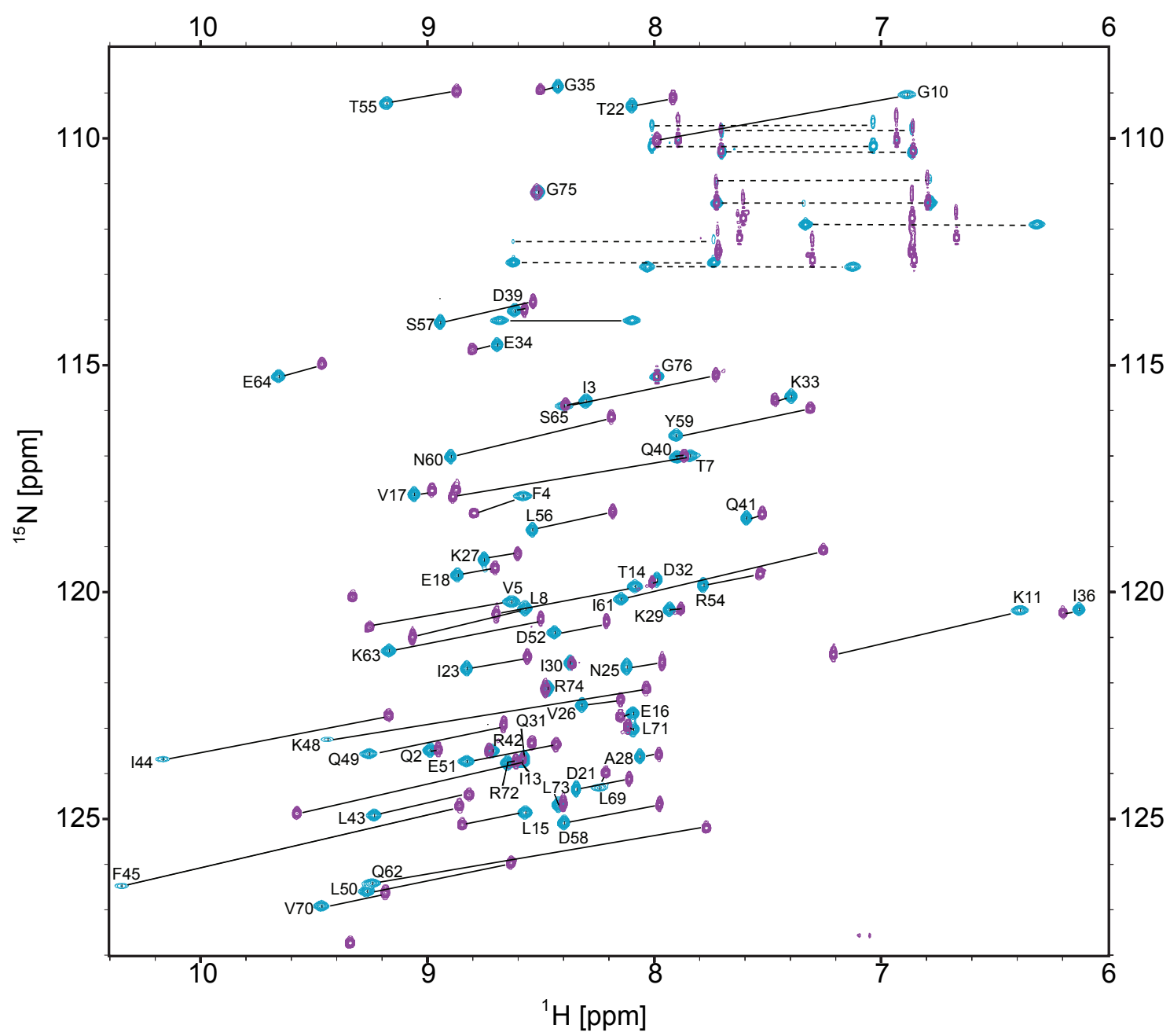

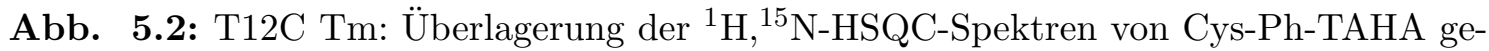
taggtem Ubiquitin-T12C, beladen mit $\mathrm{Tm}^{3+}$ (hellblau) und $\mathrm{Lu}^{3+}$ (violett). PCSs sind mit durchgezogenen Linien eingezeichnet, Signale von Seitenketten sind durch unterbrochene Linien angezeigt. Die gefalteten Signale (States-TPPI) der Aminosäurereste K6, T9 und S20 sind ausgeblendet. Eine vollständige Liste der chemischen Verschiebungen der insgesamt 65 PCSs ist im Anhang in Tabelle 7.3 gegeben. 


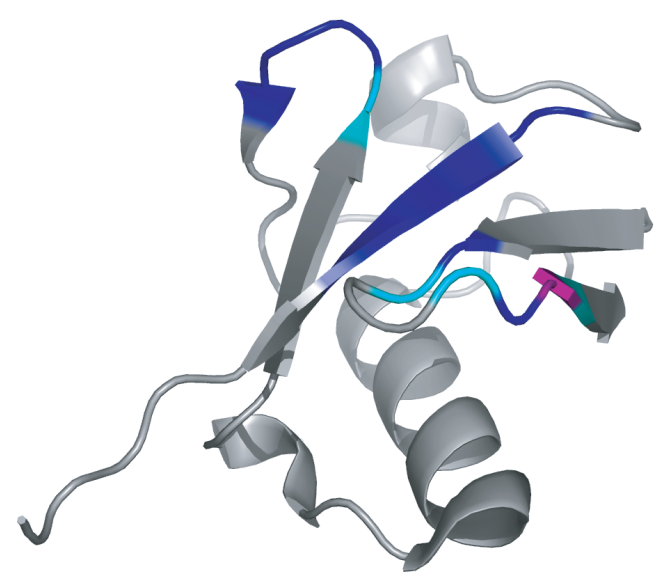

Abb. 5.3: Schematische Darstellung der Aminosäurereste, deren Signale bei T12C Tb durch PRE nicht mehr detektierbar waren (dunkelblau). Zusätzlich sind die angrenzenden Reste (T7, G10, I13 und F45), die schwache Signale zeigten, markiert (cyan). C12 (magenta) ist Teil des $\beta$-Faltblatts.

Die PCSs wurden mit einem MATLAB Skript von Dr. Mitcheell Maestre Martinez ausgewertet. Mit diesem Skript wurde die Position des Lanthanoidions, sowie der Alignmenttensor bestimmt und daraus die PCSs zurück berechnet. Dafür wurde in der Rechnung ein Raster über die Struktur gelegt und das Metallion entlang dieses Gitters bewegt (grid search). Für jede Position wurden für die experimentellen PCSs nach der Methode der kleinsten Quadrate (least square fit) jeweils der Tensor und der $Q$-Faktor ermittelt und auf diese Weise nach dem kleinsten $Q$-Faktor gesucht. Aus den zehn Strukturen der NMR-Struktur (1D3Z) wurde ein gemittelter Tensor bestimmt.

Die Auftragungen der experimentellen und berechneten ${ }^{1} \mathrm{H}$ - und ${ }^{15} \mathrm{~N}-\mathrm{PCSs}$ gegen die Aminosäurereste sind für die T12C-Mutante in Abbildung 5.4 gezeigt. Die Kurvenverläufe sind, sowohl für die experimentellen Daten in Bezug auf die zurück berechneten, als auch die der PCSs von ${ }^{1} \mathrm{H}$ und ${ }^{15} \mathrm{~N}$ zueinander, nahezu identisch. Die gute Qualität der Daten wird auch durch die kleinen Q-Faktoren von 0.19 für T12C Tb, sowie 0.15 für T12C Tm deutlich (siehe Tabelle 5.1). Die größten Abweichungen in beiden Auftragungen weisen die Aminosäurereste F4-K11 und Q62-L69 auf. Diese befinden sich allesamt in der Nähe der berechneten Ionenposition, welche in Abbil- 


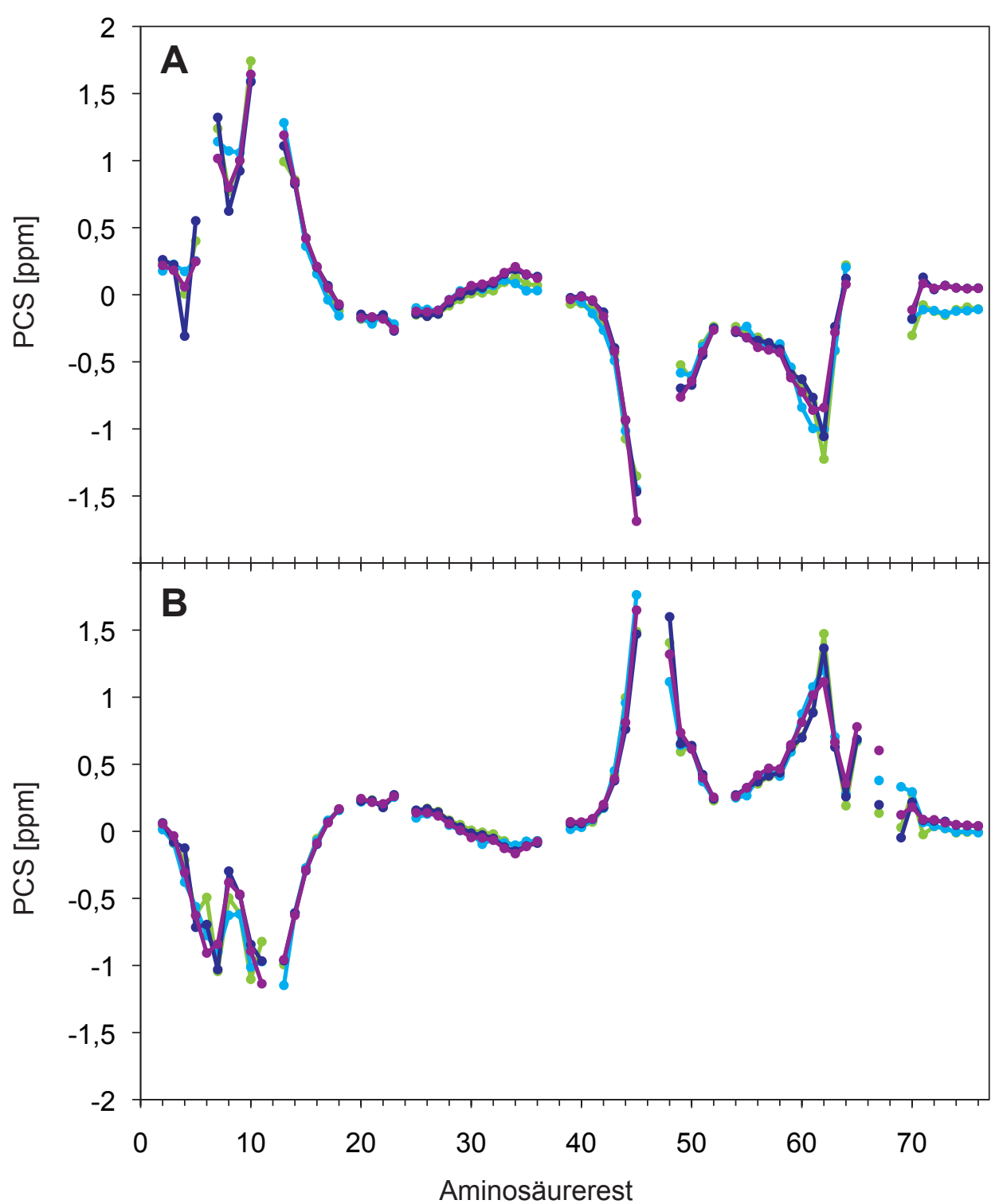

Abb. 5.4: Auftragungen der experimentellen und berechneten PCSs der T12C Mutante, beladen mit $\mathrm{Tb}^{3+}(\mathrm{A})$ und $\mathrm{Tm}^{3+}(\mathrm{B})$, gegen die Aminosäurereste. Die diamagnetische Referenzprobe war T12C Lu. Die Daten sind wie folgt gekennzeichnet: ${ }^{1} \mathrm{H}$ (exp): grün, ${ }^{1} \mathrm{H}$ (ber): dunkelblau, ${ }^{15} \mathrm{~N}(\exp )$ : hellblau, ${ }^{15} \mathrm{~N}$ (ber): violett. Die vollständigen Daten sind im Anhang in den Tabellen 7.2 und 7.3 angegeben. 

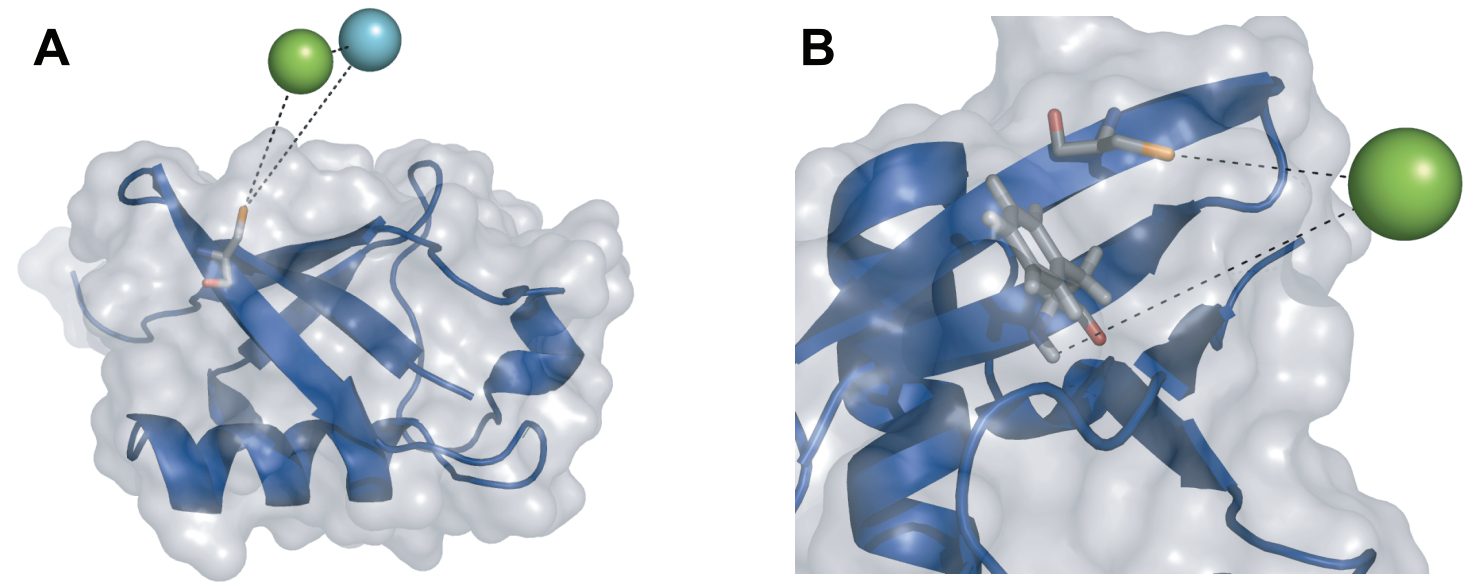

Abb. 5.5: Darstellung von T12C mit den berechneten Metallpositionen auf Basis der NMR-Struktur 1D3Z. A: Die Metallabstände vom Schwefel des C12 betragen $8.8 \AA$ für $\mathrm{Tb}^{3+}$ (grün) und $12.8 \AA$ für $\mathrm{Tm}^{3+}$ (hellblau). Die berechneten Ionenpositionen liegen $5.5 \AA$ von einander entfernt. B: Vergrößerung aus T12C Tb. Der Abstand vom F4-Amidproton zum $\mathrm{Tb}^{3+}$ beträgt $13.0 \AA$.

dung 5.5 (A) gezeigt ist. In den HSQC-Spektren (Abbildungen 5.1 und 5.2) sind einige Reste zu sehen, wie beispielsweise F4 in T12C Tb oder L69 in T12C Tm, die starke Unterschiede der PCSs in ${ }^{1} \mathrm{H}$ und ${ }^{15} \mathrm{~N}$ aufweisen. Beispielhaft ist $\mathrm{F} 4$ in einer vergrößerten Darstellung in Abbildung 5.5 (B) gezeigt. Zwar zeigt der NH-Vektor nicht direkt in Richtung des Ions, was unterschiedliche PCSs in beiden Dimensionen erklären könnte, jedoch beträgt der Abstand vom Amidproton zum Terbiumion lediglich 13.0 A. Metallnahe Aminosäurereste reagieren verstärkt auf Bewegungen des Tags und einer damit verbundenen Änderung der PCSs-Isoflächen. Darüber hinaus ist auch nicht auszuschließen, dass durch den Tag kleine lokale Veränderungen in der Struktur verursacht werden und somit die zurück berechneten PCSs fehlerbehaftet sind. Die Daten zeigen jedoch, dass ein solcher Effekt, sofern vorhanden, auf die unmittelbare Umgebung beschränkt wäre, da keine global induzierten Strukturänderungen auszumachen waren. Auffällig waren für T12C Tb auch die C-terminalen Reste L71-G76. Obwohl für beide Kerne die Werte der experimentellen PCSs in sehr guter Übereinstimmung waren, so waren sie laut der berechneten PCSs jeweils zwischen 0.16-0.21 ppm zu klein. Erhöhte Beweglichkeit führt zu einer dynamisch bedingten Reduktion der Pseudokontaktverschiebungen. Allerdings scheinen für die- 
sen Erklärungsansatz die Effekte bei T12C Tb zu groß zu sein. Laut Literatur ragen lediglich die letzten drei Aminosäurereste aus der Struktur heraus, während L71 noch zum $\beta$-Faltblatt gehört. Somit wären größere Auswirkungen auf G76 als auf L71 zu erwarten. Mit der T12C Tm Probe waren am C-Terminus keine derartigen Abweichungen der experimentellen PCSs von den berechneten auszumachen. Auch S57C Tb und S57C Tm (im Folgenden vorgestellt) zeigten keine derartigen Unterschiede. 


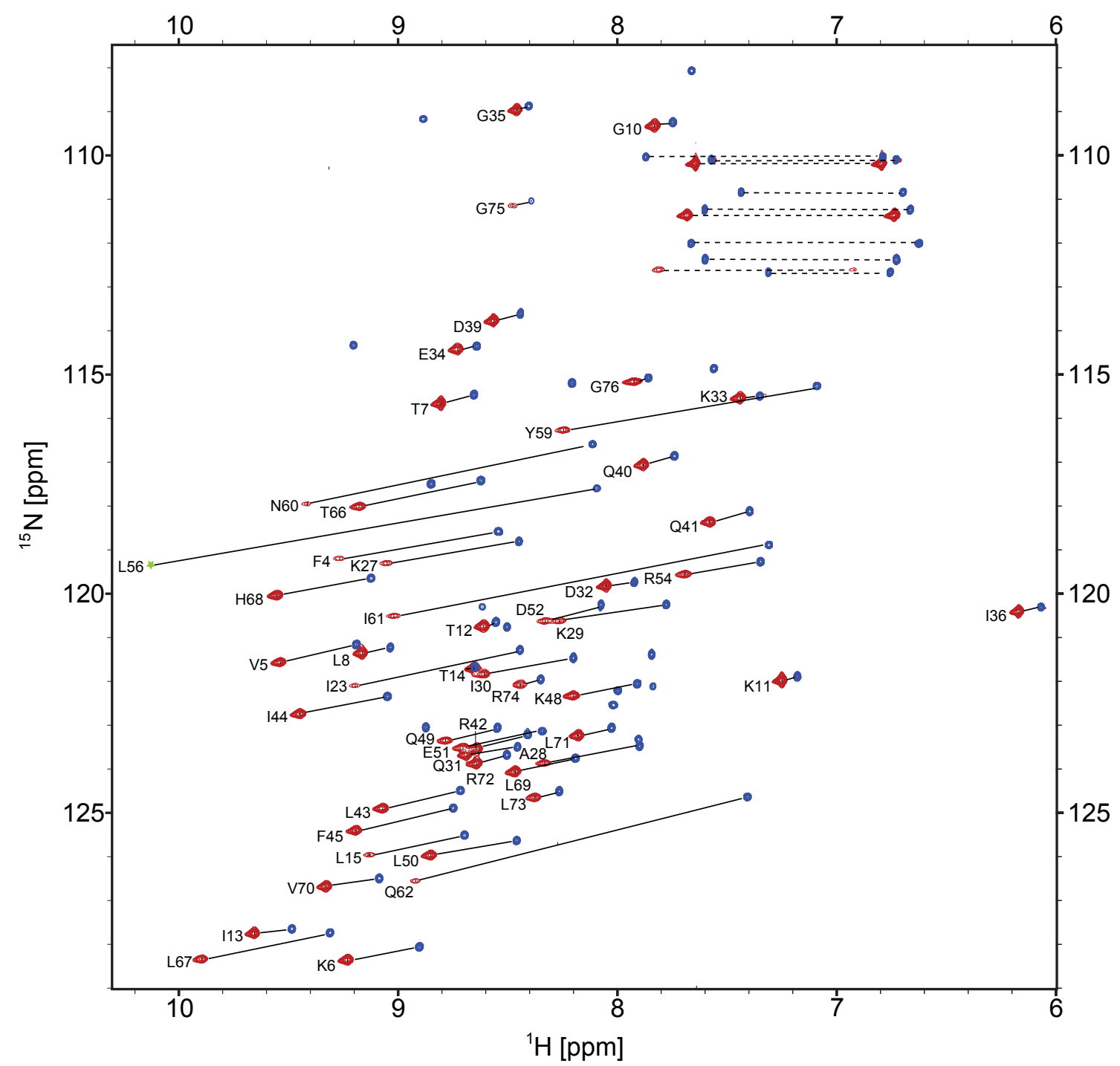

Abb. 5.6: S57C Tb: Überlagerung der ${ }^{1} \mathrm{H},{ }^{15} \mathrm{~N}-\mathrm{HSQC}-$ Spektren von Cys-Ph-TAHA getaggtem Ubiquitin-S57C, beladen mit $\mathrm{Tb}^{3+}$ (rot) und $\mathrm{Lu}^{3+}$ (dunkelblau). PCSs sind mit durchgezogenen Linien eingezeichnet, Signale von Seitenketten sind durch unterbrochene Linien angezeigt. Die gefalteten Signale (StatesTPPI) der Aminosäurereste T9, A46 und G47 sind ausgeblendet. Der Peak von L56 ist schwach und mit einem Stern (grün) markiert. Eine vollständige Liste der chemischen Verschiebungen der insgesamt 54 PCSs ist im Anhang in der Tabelle 7.4 gegeben. 


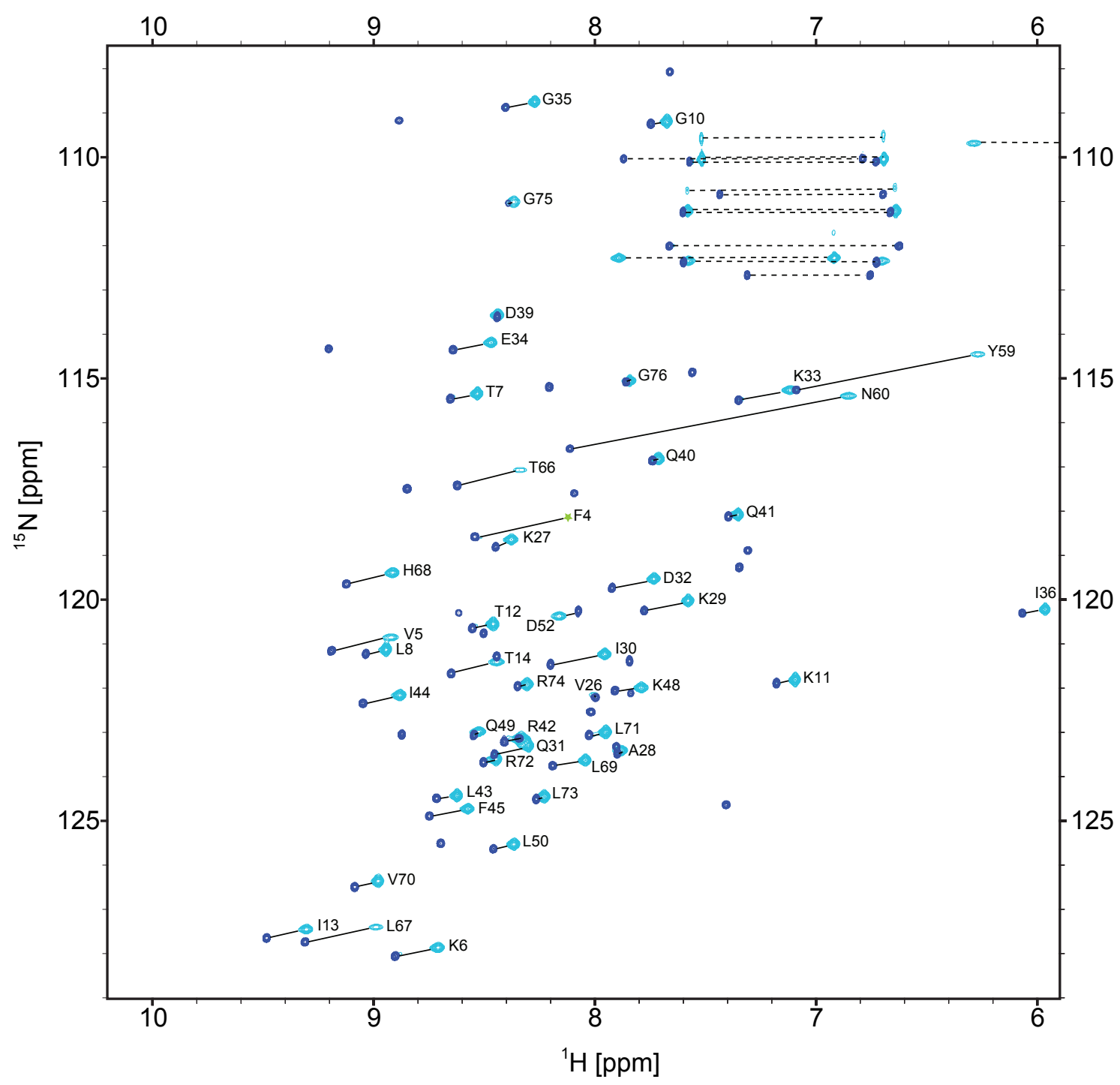

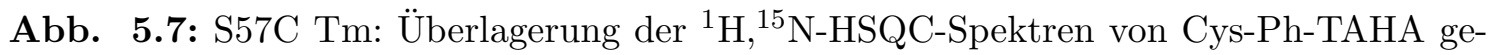
taggtem Ubiquitin-S57C, beladen mit $\mathrm{Tm}^{3+}$ (hellblau) und $\mathrm{Lu}^{3+}$ (dunkelblau). PCSs sind mit durchgezogenen Linien eingezeichnet, Signale von Seitenketten sind durch unterbrochene Linien angezeigt. Die gefalteten Signale (States-TPPI) der Aminosäurereste T9, A46 und G47 sind ausgeblendet. Der Peak von F4 ist schwach und mit einem Stern (grün) markiert. Eine vollständige Liste der chemischen Verschiebungen der insgesamt 48 PCSs ist im Anhang in der Tabelle 7.5 gegeben. 
Die Untersuchungen wurden mit einer zweiten Ubiquitinmutante (S57C) wiederholt. C57 befindet sich in dem kleinen $\alpha$-helicalen Bereich, der sich von L56-N60 erstreckt und von zwei Random Coils flankiert wird. Als diamagnetische Referenzprobe wurde S57C Lu verwendet. In den Abbildungen 5.6 und 5.7 sind die überlagerten HSQCSpektren mit S57C Tb und S57C Tm gezeigt. Die vollständigen chemischen Verschiebungen, sowie die resultierenden PCSs sind in den Tabellen 7.4 und $7.5 \mathrm{im}$ Anhang angegeben. In den beiden anisotropen Proben waren insgesamt weniger Signale detektierbar, als bei den entsprechenden T12C Proben. Für S57C Tb wurden 54 PCSs gefunden, während bei S57C Tm sogar nur 48 PCSs messbar waren. Die Reste, die in S57C Tm durch PRE ausgelöscht waren, sind in Abbildung 5.8 blau dargestellt. Darüber hinaus sind die Aminosäurereste, deren Peaks bereits deutlich verbreitert und dementsprechend schwach waren (F4, I44, F45 und K48), hellblau markiert. Bemerkenswert ist die Tatsache, dass entgegen der Erwartung mit der Terbium-markierten Probe mehr Signale erhalten wurden, als mit Thulium. Dies lässt sich jedoch anhand der berechneten Metallpositionen erklären (siehe Abbildung 5.9). Beide Ionen sind sehr nahe an der Proteinoberfläche lokalisiert, was in guter Übereinstimmung mit der reduzierten Signalanzahl im Vergleich zu T12C ist. Während die Terbiumposition noch vollständig außerhalb des Proteins liegt, befindet sich das Thuliumion in direktem Kontakt mit der Oberfläche. Der Abstand vom

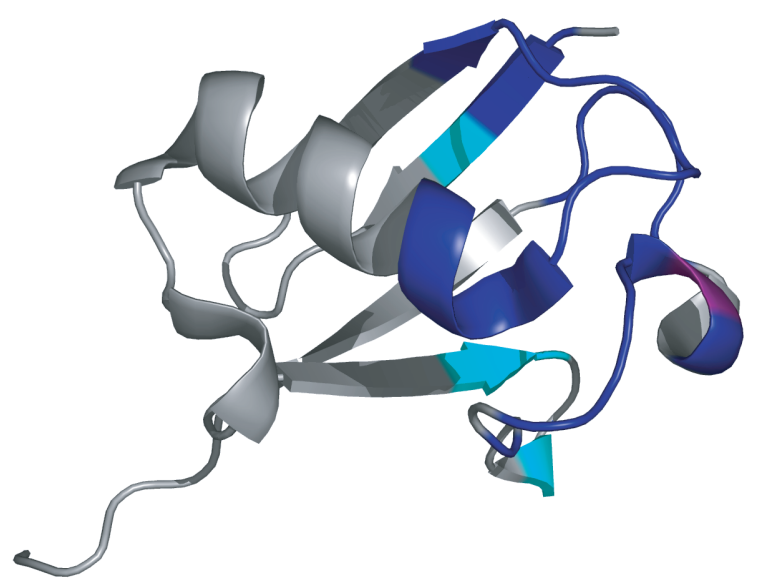

Abb. 5.8: Schematische Darstellung der Aminosäurereste, deren Signale bei S57C Tm durch PRE nicht mehr detektierbar waren (dunkelblau). Die angrenzenden Reste mit schwachen Signalen sind hellblau gezeigt, C57 ist magenta markiert. 


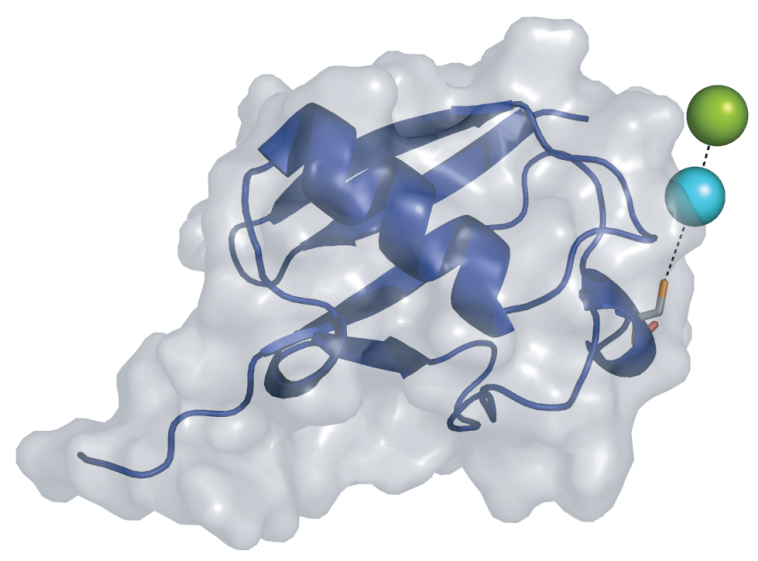

Abb. 5.9: Darstellung der berechneten Metallpositionen. Der Abstand vom Schwefel des C57 beträgt $5.8 \AA$ für $\mathrm{Tm}^{3+}$ (hellblau) und $11.0 \AA$ für $\mathrm{Tb}^{3+}$ (grün). Beide berechneten Ionenpositionen liegen sehr nahe an der Proteinoberfläche, auch wenn RDCs mit in die Berechnungen einbezogen werden (siehe Abschnitt 5.4).

Thuliumion zum Schwefel des C57 beträgt lediglich $5.8 \AA$, während das Terbiumion beinahe in direkter Verlängerung weitere $5.8 \AA$ entfernt liegt. Besonders die Distanz zum Thuliumion scheint deutlich zu kurz für den Cys-Ph-TAHA-Tag zu sein. Obwohl dieser über einige flexible Bindungen verfügt, wäre doch eine massive Biegung des Tags von Nöten. Die Ubiquitinoberfläche weist an dieser Stelle eine negative Ladung auf (E18). Da Cys-Ph-TAHA durch die sechs Carboxylfunktionen formal dreifach negativ geladen ist, ist eine elektrostatische Interaktion unwahrscheinlich. Darüber hinaus würde sich eine derartige Stabilisierung der Metallposition in großen RDCs zeigen. Dies ist für S57C jedoch nicht der Fall (vergleiche Abschnitt 5.3). Eine mögliche Erklärung für die berechneten Positionen wäre, dass die Mittelung über einen abgeflachten Kegel erfolgte, wodurch die berechnete Metallposition sehr nahe an das Protein kommen würde. Dies wäre ebenfalls im Einklang mit der reduzierten Signalanzahl, da durch einen vergrößerten Bewegungsradius des Tags mehr Kerne in den Einflussbereich des paramagnetischen Ions geraten und so die $\mathrm{r}^{-6}$ abhängige PRE überbetont wird.

Für S57C wurden ebenfalls die experimentellen und zurück berechneten ${ }^{1} \mathrm{H}$ - und ${ }^{15}$ N-PCSs gegen die Aminosäurereste aufgetragen (Abbildung 5.10). In beiden Proben stimmten die experimentellen Werte hervorragend mit den berechneten über- 


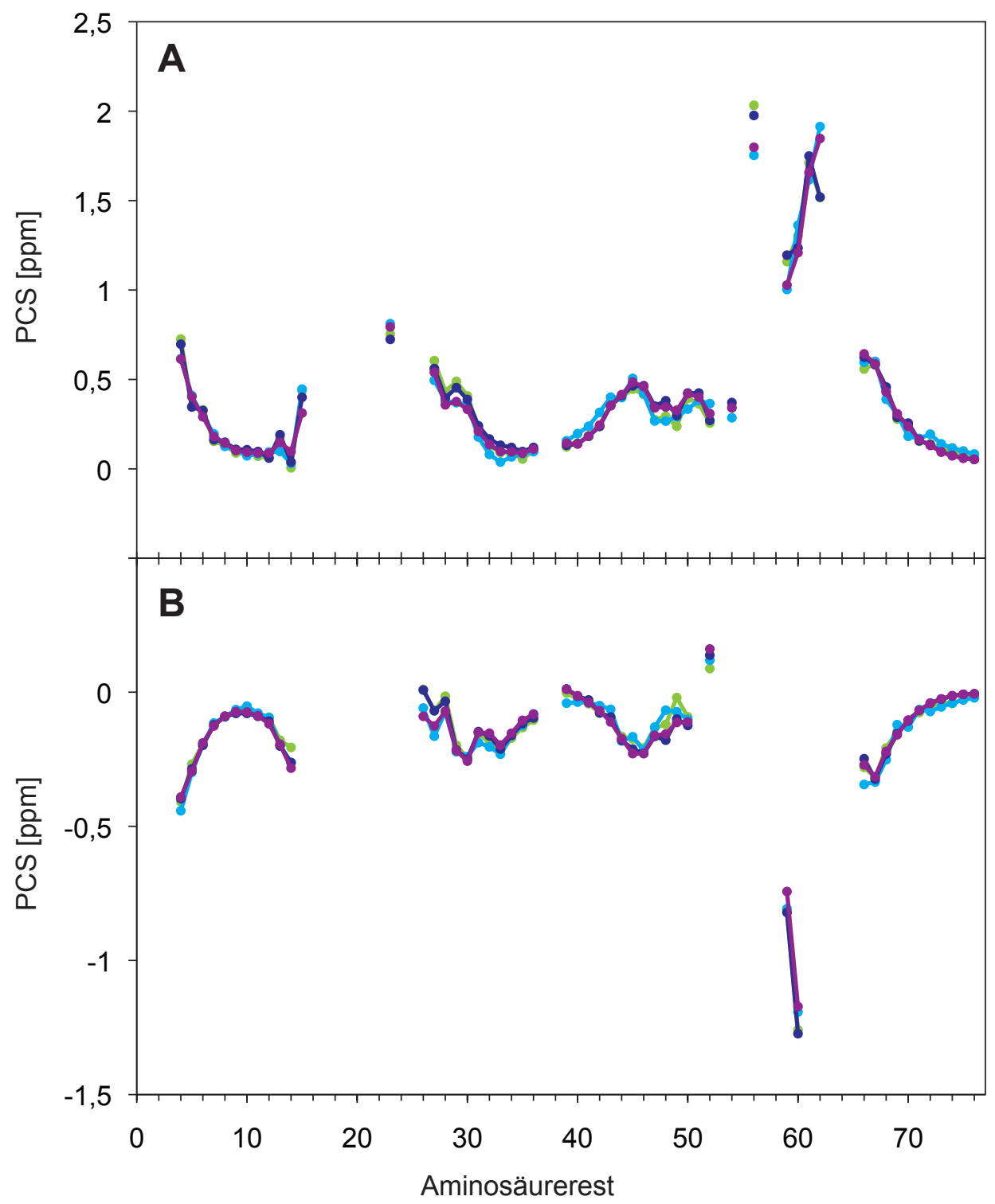

Abb. 5.10: Auftragungen der experimentellen und berechneten PCSs der S57C Mutante, beladen mit $\mathrm{Tb}^{3+}(\mathrm{A})$ und $\mathrm{Tm}^{3+}(\mathrm{B})$, gegen die Aminosäurereste. Die diamagnetische Referenzprobe war S57C Lu. Die Daten sind wie folgt gekennzeichnet: ${ }^{1} \mathrm{H}$ (exp): grün, ${ }^{1} \mathrm{H}$ (ber): dunkelblau, ${ }^{15} \mathrm{~N}(\exp )$ : hellblau, ${ }^{15} \mathrm{~N}$ (ber): violett. Die vollständigen Daten sind im Anhang in den Tabellen 7.4 und 7.5 angegeben. 
Tab. 5.1: Zusammenfassung der Ergebnisse der PCS Analysen. N gibt die Anzahl der PCSs an, $Q$ den $Q$-Faktor und R den Korrelationsfaktor. $\Delta \chi_{a x}$ und $\Delta \chi_{r h}$ sind die axiale bzw. rhombische Komponente des magnetischen Suszeptibilitätstensors $\left[10^{-32} \mathrm{~m}^{3}\right]$. Die Eulerwinkel $\alpha, \beta, \gamma\left[^{\circ}\right]$ beschreiben die Rotation des Tensors in das Hauptkoordinatensystem des Proteins. Zusätzlich sind die jeweils größten experimentell bestimmten PCSs aufgeführt.

\begin{tabular}{|c|c|c|c|c|c|c|c|c|c|c|}
\hline \multirow[b]{2}{*}{ Probe } & \multirow[b]{2}{*}{$\mathrm{N}$} & \multirow[b]{2}{*}{$Q$} & \multicolumn{4}{|c|}{ größte PCSs [ppm] } & \multirow[b]{2}{*}{$\Delta \chi_{r h}$} & \multirow[b]{2}{*}{$\alpha$} & \multirow[b]{2}{*}{$\beta$} & \multirow[b]{2}{*}{$\gamma$} \\
\hline & & & $\mathrm{R}^{2}$ & neg. & pos. & $\Delta \chi_{a x}$ & & & & \\
\hline $\mathrm{T} 12 \mathrm{C} \mathrm{Tb}$ & 59 & 0.19 & 0.97 & -1.352 & 1.743 & -14.68 & -1.82 & 141.34 & 90.73 & -22.89 \\
\hline $\mathrm{T} 12 \mathrm{C} \mathrm{Tm}$ & 65 & 0.15 & 0.98 & -1.103 & 1.490 & -13.17 & -3.99 & 5.63 & 72.33 & 100.30 \\
\hline $\mathrm{S} 57 \mathrm{C} \mathrm{Tb}$ & 54 & 0.07 & 0.99 & - & 2.033 & -12.66 & -8.25 & -19.92 & 82.18 & -21.96 \\
\hline S57C Tm & 48 & 0.11 & 0.98 & -1.259 & 0.088 & 8.44 & 1.21 & -139.51 & 47.95 & -12.45 \\
\hline
\end{tabular}

ein, was sich in den sehr kleinen Q-Faktoren von 0.07 für S57C Tb und 0.11 für S57C Tm ausdrückt. Wie auch schon bei der T12C-Mutante zu sehen war, verlaufen auch die Auftragungen von S57C annähernd spiegelbildlich zueinander. Auffällig ist, dass für S57C Tb ausschließlich positive PCSs von bis zu 2 ppm gemessen wurden, während für S57C Tm fast alle Werte negativ waren (siehe Tabelle 5.1). Die axialen Komponenten $\Delta \chi_{a x}$ der beiden Proben von S57C waren jeweils kleiner, als die der korrespondieren T12C-Proben. Es zeigt, dass der Tag an C57 gebunden mehr Freiheitsgrade aufweist, als an C12. 


\subsection{Temperaturverhalten von T12C Tm}

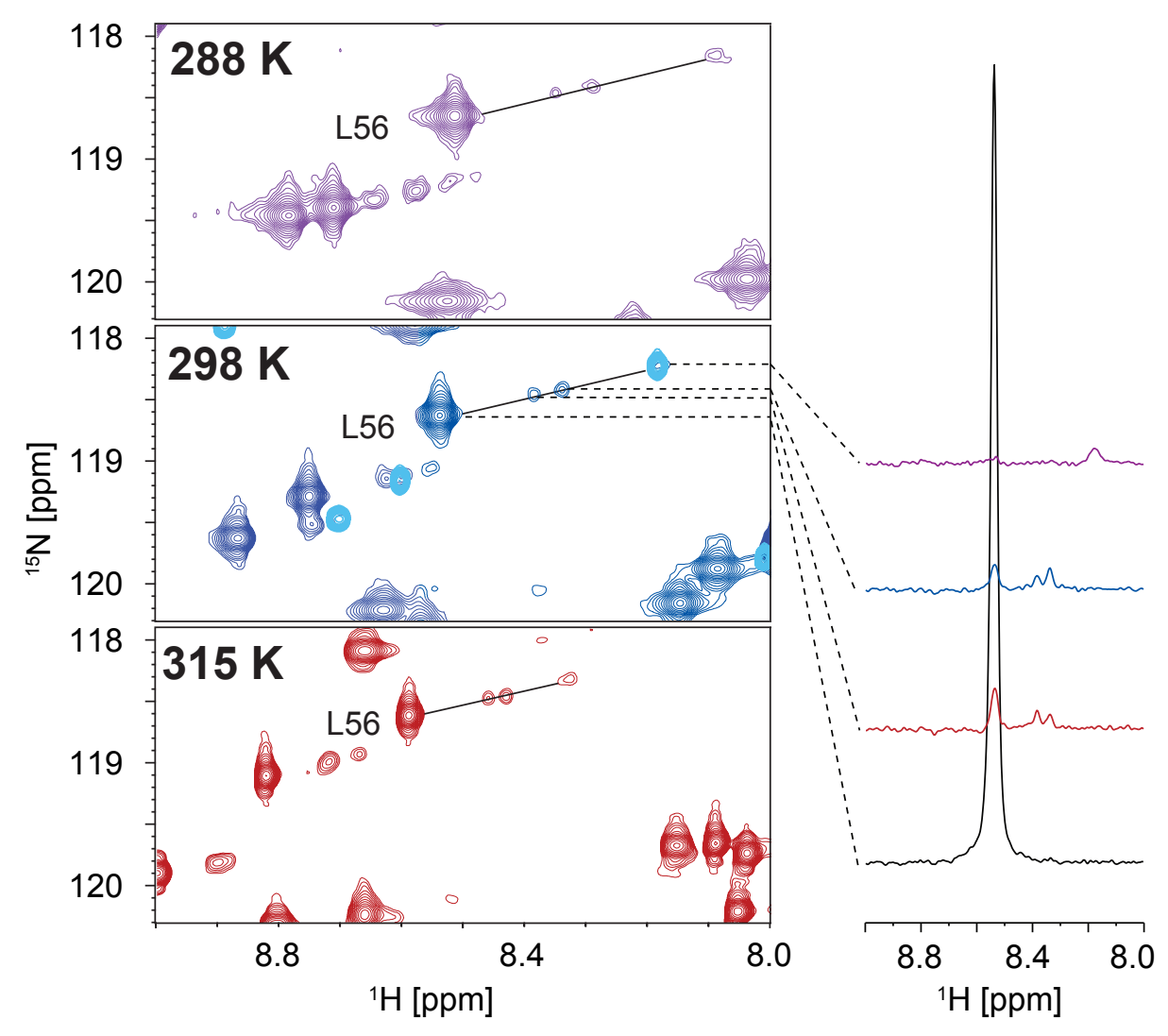

Abb. 5.11: T12C Tm: Ausschnitte aus den ${ }^{1} \mathrm{H},{ }^{15} \mathrm{~N}-\mathrm{HSQC}-S p e k t r e n$ der T12C-Mutante, beladen mit $\mathrm{Tm}^{3+}$, bei verschiedenen Temperaturen. Die Spektren sind auf einem niedrigen Contourlevel abgebildet, so dass die zwei zusätzlichen, anisotropen Signalsätze zu sehen sind. Die Farben sind wie folgt zugeordnet: 288 K: violett, 298 K: dunkelblau, 315 K: rot. Das Spektrum bei 298 K ist mit der isotropen Referenzprobe (hellblau) überlagert. Die PCS ist für den Aminosäurerest L56 eingezeichnet. Zur Verdeutlichung der relativen Peakintensitäten sind die 1D-Spuren von L56 bei $298 \mathrm{~K}$ gezeigt.

Neben den zuvor diskutierten, paramagnetisch verschobenen Signalen, waren in den HSQC-Spektren von T12C Tb und T12C Tm für einige Aminosäurereste Peaks zweier zusätzlicher, anisotroper Datensätze zu sehen. Diese waren, wie weiter unten quantifiziert, mit jeweils etwa $2 \%$ relativ schwach und nur bei sehr niedrigem Contourlevel auszumachen. In den meisten Fällen lagen diese in gerader Linie zwischen dem isotropen Peak und dem anisotropen Hauptpeak. Für den DOTA-M8-Tag 
wurde ebenfalls ein zweiter Signalsatz beschrieben, dessen Beitrag von 15-20\% bei $298 \mathrm{~K}$ auf 50\% bei $323 \mathrm{~K}$ zunahm (Häussinger et al. (2009)). Es wurde deshalb untersucht, ob auch bei Cys-Ph-TAHA-getaggten Proben eine Temperaturabhängigkeit der relativen Peakintensitäten vorliegt. Dazu wurden fünf ${ }^{1} \mathrm{H},{ }^{15} \mathrm{~N}-\mathrm{HSQC}-$ Spektren im Bereich von 278-315 K aufgenommen (Die Temperatur von $323 \mathrm{~K}$ war mit dem verwendeten Cryoprobenkopf nicht zu erreichen). In Abbildung 5.11 sind Ausschnitte der Spektren gezeigt. Beispielhaft wurde für den Aminosäurerest L56 die PCS eingezeichnet. Das Spektrum bei $298 \mathrm{~K}$ ist mit dem Spektrum von T12C Lu überlagert, wodurch die isotropen Restsignale identifiziert werden konnten. Um die Anteile der zusätzlichen Signale zu quantifizieren, wurden zehn Aminosäurereste ausgewählt, deren Peaks zwischen $288 \mathrm{~K}$ und $315 \mathrm{~K}$ hinreichend aufgelöst waren. Das bei $278 \mathrm{~K}$ aufgenommene HSQC wurde nicht mit berücksichtigt, da die zusätzlichen Signalsätze zu stark verbreitert waren. Aus dem Verhältnis der Peakintensitäten wurden die prozentualen Anteile bestimmt. In Tabelle 5.2 sind die Ergebnisse zusammengefasst. Es ist zu sehen, dass in dem beschriebenen Bereich keine temperaturabhängige Änderung der Peakintensitäten erfolgte. Die Anteile der zusätzlichen Signalsätze blieben, ebenso wie die der isotropen Peaks, bei etwa $2 \%$, während der Hauptpeak stets zu etwa $94 \%$ vertreten war. Das relative Intensitätsverhältnis vom

Tab. 5.2: T12C Tm: Bestimmung der prozentualen Verteilungen der Peakintensitäten bei verschiedenen Temperaturen $[K]$. Neben dem Hauptpeak sind in der Probe isotrope Restsignale und Peaks von zwei zusätzlichen, anisotropen Signalsätzen zu sehen. Die zugrunde liegenden vollständigen Berechnungen sind in den Tabellen 7.6 und $7.7 \mathrm{im}$ Anhang zu finden.

\begin{tabular}{ccccc}
\hline & & \multicolumn{3}{c}{ zusätzliche Signale } \\
$\mathrm{T}$ & Hauptpeak & isotrop & anisotrop 2 & anisotrop 3 \\
\hline 288 & 93.79 & 2.48 & 1.78 & 1.95 \\
298 & 93.90 & 2.31 & 1.84 & 1.95 \\
308 & 94.19 & 2.11 & 2.01 & 1.69 \\
315 & 93.87 & 2.15 & 1.97 & 2.01 \\
\hline
\end{tabular}


Hauptpeak zu den kleinen Signalen beträgt somit jeweils lediglich 49:1, weshalb der Einfluss der zusätzlichen Peaksätze auf den Hauptpeak zu vernachlässigen war.

Wie bereits in Kapitel 2.2 erwähnt, sind die PCSs abhängig von der Temperatur. Für die Zuordnung wurden die fünf ${ }^{1} \mathrm{H},{ }^{15} \mathrm{~N}-\mathrm{HSQC}-S p e k t r e n$ von $278-315 \mathrm{~K}$ überlagert. Die einzelnen Aminosäurereste zeigten jeweils eine nahezu lineare Signalverschiebung (siehe Abbildung 5.12), wodurch der Verlauf einfach zu verfolgen war. Von der isotropen Referenz T12C Lu wurden ebenfalls HSQC-Spektren von 278-315 K aufgenommen. Mit diesen Daten wurden dann die PCSs in Abhängigkeit von der Temperatur bestimmt. Die experimentellen ${ }^{1} \mathrm{H}$ - und ${ }^{15} \mathrm{~N}$-PCSs wurden gegen die Aminosäurereste aufgetragen (siehe Abbildung 5.14). Insbesondere die Reste, die in der Struktur nahe am Lanthanoidion liegen, zeigten deutliche Effekte. Beispielhaft seien die Unterschiede der ${ }^{1} \mathrm{H}-\mathrm{PCS}$ von Q62 genannt, die sich von 1.1 auf 2.0 ppm beinahe verdoppelten. Alle zugehörigen Werte sind im Anhang in den Tabellen 7.8-7.10 angegeben. Mit dem MATLAB Skript von Dr. Mitcheell Maestre Martinez wurden wiederum die Metallpositionen ermittelt. Für alle Temperaturen wurden sehr ähnliche Positionen gefunden. Diese sind zusammen mit der NMR-Struktur (PDB-Kode 1D3Z) in Abbildung 5.13 gezeigt. Dieses Ergebnis unterstreicht die Anwendbarkeit von Cys-Ph-TAHA über einen weiten Temperaturbereich.

Die Größe der PCSs nimmt mit sinkender Temperatur zu. Gleichzeitig werden allerdings auch die Linien breiter, so dass für die PCS-Messungen ein Kompromiss zwischen Linienschärfe und Größe des Effekts gefunden werden muss. Der Temperaturbereich zwischen von 288 und $298 \mathrm{~K}$ scheint für Ubiquitin ideal zu sein. Bei der Anwendung des Tags auf größere Proteine und der damit verbundenen stärkeren spektralen Überlappung, empfehlen sich jedoch Messungen bei höherer Temperatur zu Gunsten einer höheren Empfindlichkeit. Wie bereits erwähnt, traten die zuvor beschriebenen, zusätzlichen Signalsätze auch in der T12C Tb Probe in ähnlichen prozentualen Anteilen auf, während keine weiteren anisotropen Signale für S57C Tb oder S57C Tm zu sehen waren. Für S57C Tm wurden ebenfalls HSQC-Spektren zwischen $278 \mathrm{~K}$ und $315 \mathrm{~K}$ aufgenommen. Auch hier traten keine Veränderungen der relativen Peakintensitäten auf. Was die Ursache der zusätzlichen Peaks in den T12CProben war, konnte nicht aufgeklärt werden. Da das Auftreten jedoch abhängig von 


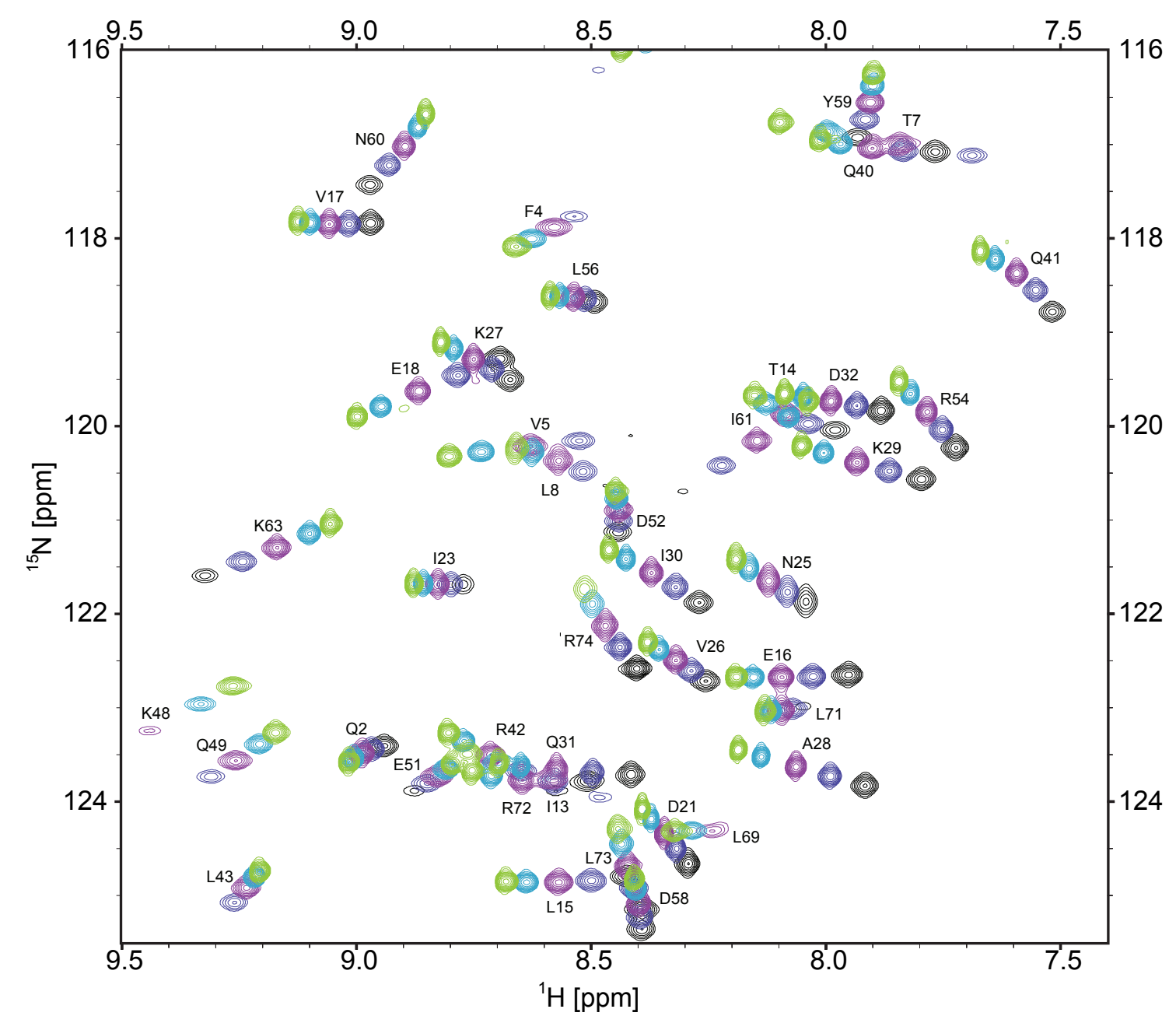

Abb. 5.12: T12C Tm: Ausschnitt aus den überlagerten ${ }^{1} \mathrm{H},{ }^{15} \mathrm{~N}-\mathrm{HSQC}-$ Spektren bei verschiedenen Temperaturen. Die Farben sind folgend zugeordnet: $278 \mathrm{~K}$ : schwarz, $288 \mathrm{~K}$ : dunkelblau, $298 \mathrm{~K}$ : violett, $308 \mathrm{~K}$ : hellblau, $315 \mathrm{~K}$ : grün. Eine vollständige Liste der chemischen Verschiebungen ist im Anhang in der Tabelle 7.9 gegeben. 


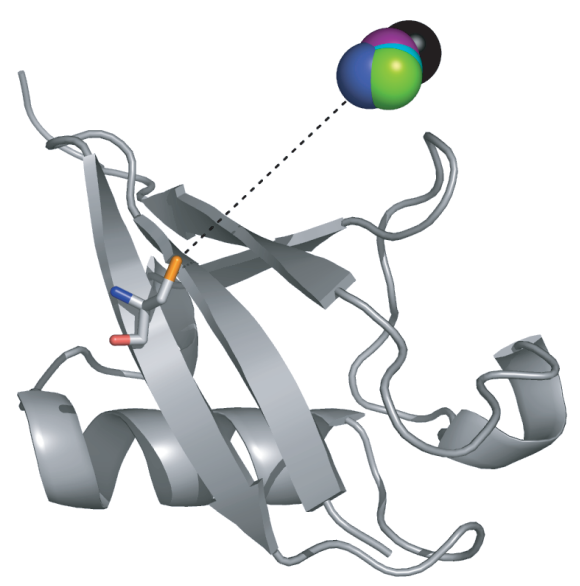

Abb. 5.13: Berechnete Metallpositionen in T12C Tm bei unterschiedlichen Temperaturen auf Basis der NMR-Struktur (1D3Z). Die Farben sind folgend zugeordnet: $278 \mathrm{~K}$ : schwarz, $288 \mathrm{~K}$ : dunkelblau, $298 \mathrm{~K}$ : violett, $308 \mathrm{~K}$ : hellblau, $315 \mathrm{~K}$ : grün.

der Ubiquitinmutante war, lässt sich schlussfolgern, dass nicht diastereomere Lanthanoidkomplexe von Cys-Ph-TAHA die Ursache waren, sondern Interaktionen mit dem Protein. Da die Anteile von 2\% sehr gering waren und die Zusatzpeaks somit die Spektren nicht bedeutend beeinflusst haben, wurden sie im Folgenden ignoriert. 


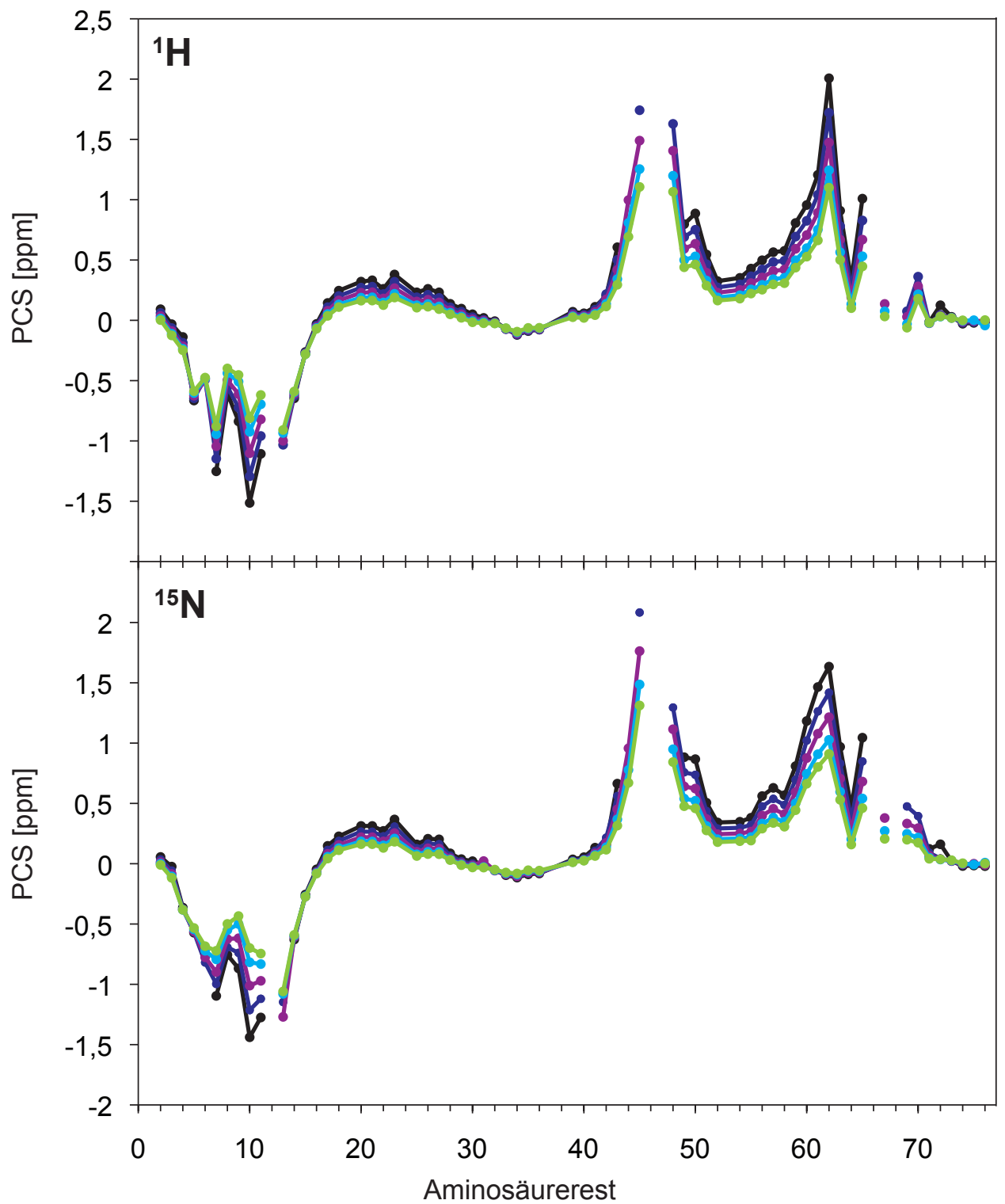

Abb. 5.14: Auftragungen der experimentellen $\operatorname{PCSs}\left({ }^{1} \mathrm{H}\right.$ : oben, ${ }^{15} \mathrm{~N}$ : unten $)$ von T12C Tm gegen die Aminosäurereste bei verschiedenen Temperaturen. Die diamagnetische Referenzprobe war T12C Lu. Die Farben sind folgend zugeordnet: $278 \mathrm{~K}$ : schwarz, $288 \mathrm{~K}$ : dunkelblau, $298 \mathrm{~K}$ : violett, $308 \mathrm{~K}$ : hellblau, 315 K: grün. Vollständige Listen der chemischen Verschiebungen bei den jeweiligen Temperaturen für T12C Lu und T12C Tm sind im Anhang in den Tabellen 7.8 und 7.9 gegeben. Die resultierenden PCSs finden sich in der Tabelle 7.10. 


\subsection{Residuale dipolare Kopplungen}

Die RDCs wurden auf Grund ihrer quadratischen Abhängigkeit vom Magnetfeld mit dem stärksten zur Verfügung stehenden Magneten bei 21.1 T gemessen. Um Überlappungen der Signale zu minimieren, wurde die IPAP-HSQC-Sequenz verwendet (in-phase-antiphase-HSQC, Ottiger et al. (1998)). Mit dieser Pulssequenz können die heteronuklearen ${ }^{1} J$-NH Kopplungen in der indirekten Dimension gemessen werden, ohne dass eine Verdopplung der Signale auftritt (siehe Abschnitt 6.3).

Wie bereits in Kapitel 2.1 beschrieben, wird die Differenz der Kopplungen einer orientierten Probe und einer isotropen Referenz als residuale dipolare Kopplung bezeichnet. Ein Ausschnitt aus den beiden IPAP-Spektren ist in Abbildung 5.15 beispielhaft gezeigt. Die Probe der T12C-Mutante war zu 50\% mit Terbium beladen. Das Spektrum verdeutlicht, dass grundsätzlich bereits eine halbbeladene Probe ausreicht, um RDCs extrahieren zu können. Um spektrale Überlappungen zu minimieren, wurden jedoch möglichst die vollständig beladenen anisotropen Proben, sowie die isotrope Referenzprobe einzeln gemessen. Als Referenz wurde getaggtes,

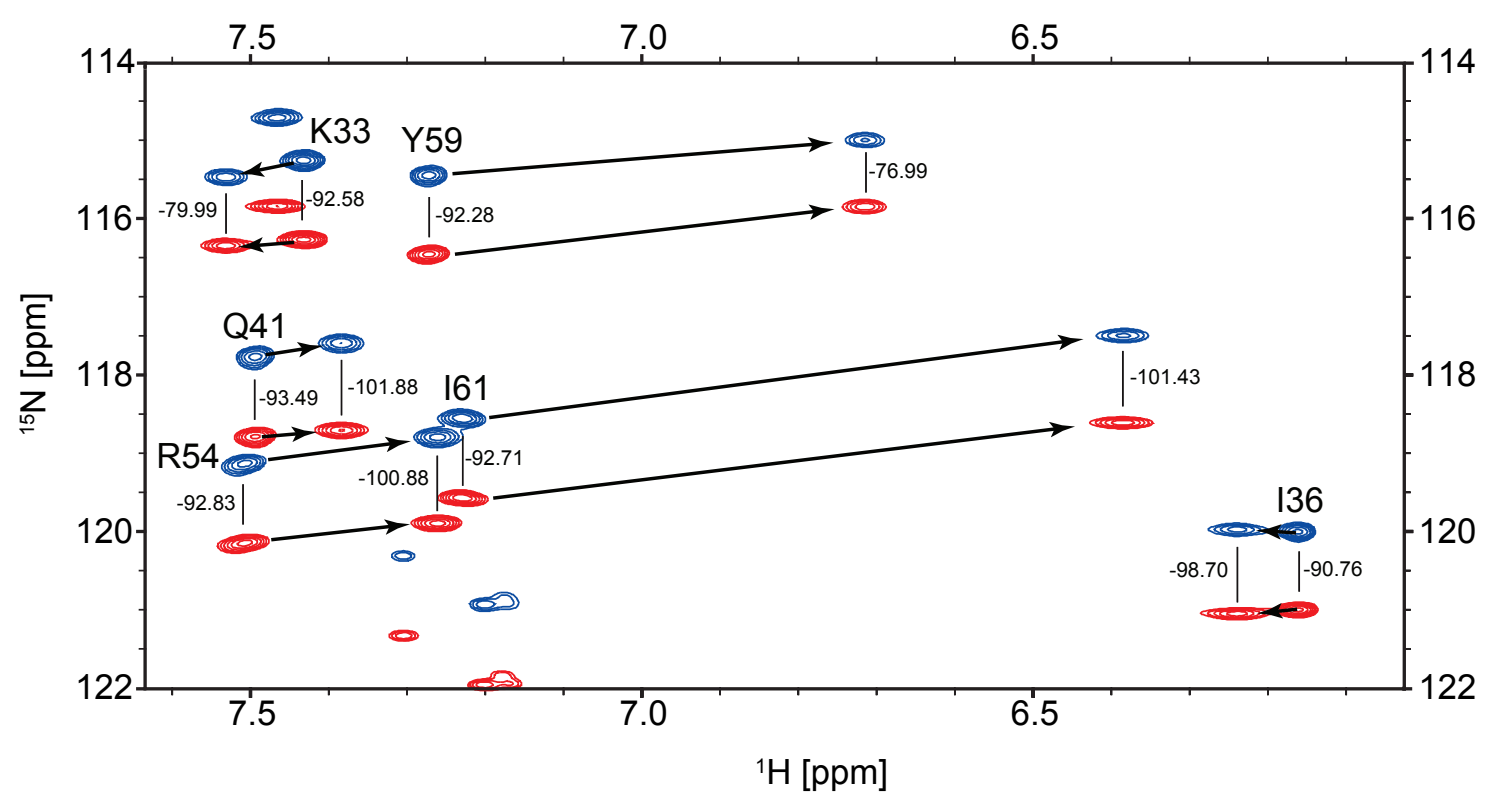

Abb. 5.15: T12C Tb: Ausschnitt aus einer Überlagerung der beiden IPAP-HSQCSpektren (Summe und Differenz). Die Probe war zu 50\% mit Terbium beladen. Für die markierten Reste sind die PCSs und die jeweiligen Amidkopplungen des orientierten und nicht-orientierten Ubiquitins gezeigt. 
aber unbeladenes Ubiquitin verwendet. Aus den extrahierten RDCs wurden mit dem Programm PALES (Zweckstetter \& Bax (2000); Zweckstetter (2008)) der jeweilige Alignmenttensor bestimmt und die RDCs zurück berechnet. Das Programm benötigt zur Berechnung als Eingabedatei eine PDB-Struktur. Hier wurde der erste von zehn Einträgen der NMR-Struktur (1D3Z, Cornilescu et al. (1998)) verwendet. In Abbildung 5.16 sind Auftragungen der experimentell ermittelten RDCs der vier anisotropen Proben gegen die zurück berechneten Werte gezeigt. Die flexiblen Reste R74-G76 wurden dabei nicht mit in die Berechnungen einbezogen. Darüber hinaus wurden Aminosäuren ausgeschlossen, deren exakte Peakposition durch Überlappung mit anderen Peaks nicht ermittelt werden konnte. Allgemein wurden die Positionen der Signale ausschließlich automatisch ohne manuelle Korrekturen bestimmt, um systematische Fehler zu minimieren. RDCs sind auf Grund der geringen Messgröße von wenigen $\mathrm{Hz}$ viel anfälliger für Fehler, als PCSs (für Stickstoff entspricht 1 ppm etwa $90 \mathrm{~Hz}$ bei $21.1 \mathrm{~T}$ ). Bereits geringe Abweichungen in der chemischen Verschiebung bewirken eine große Änderung der RDCs. Die Auftragungen zeigen eine hohe Korrelation der experimentellen RDCs mit den berechneten, was sich auch in den niedrigen $Q$-Faktoren widerspiegelt (siehe Tabelle 5.3). Hervorzuheben sind die Ergebnisse der T12C-Mutante. Zum einen wurden hier mit Terbium sehr große RDCs von bis zu $17.8 \mathrm{~Hz}$ gemessen, zum anderen wurden mit Thulium insgesamt 61 RDCs erhalten, die in den Rechnungen verwendet werden konnten. Die größten RDCs von S57C Tb und S57C Tm betrugen, verglichen mit den entsprechenden T12C Proben, weniger als 50\%, was auf eine vermehrte Dynamik hindeutet. Wie zuvor erwähnt, befindet sich C57 in einem $\alpha$-helicalen Bereich von fünf Aminosäureresten, der von zwei Random Coils flankiert wird. Entgegen der Erwartung weist das Proteinrückgrat innerhalb dieser Helix jedoch weniger Dynamik auf, als C12 in dem $\beta$-Faltblatt. Dies zeigte sich anhand der RDC-basierten Orderparameter $\mathrm{S}_{r d c}^{2}$ (Lakomek et al. (2008)). Somit beruht die erhöhte Flexibilität nicht auf intrinsischer Proteinbewegung, sondern vielmehr auf einer größeren Beweglichkeit des Tags oder der Seitenkette selbst. Bei der Diskussion der PCS-Analysen wurde bereits spekuliert, dass sich der Tag, an C57 gebunden, auf einer abgeflachten Kegelbahn bewegt, wodurch die RDCs und PCSs einer stärkeren Mittelung unterliegen. Der Vergleich der RDCs von T12C und 
Tab. 5.3: Zusammenfassung der Ergebnisse der RDC Analysen. N gibt die Anzahl der RDCs an, $Q$ den $Q$-Faktor, R den Korrelationsfaktor, $\mathrm{Da}_{N H}$ die axiale Komponente des Alignmenttensors [Hz] und Rh die Rhombizität des Tensors. Die Eulerwinkel $\alpha, \beta, \gamma\left[^{\circ}\right]$ beschreiben die Rotation des Tensors in das Hauptkoordinatensystem des Proteins. Zusätzlich sind die jeweils größten gemessenen RDCs aufgeführt. Die Werte wurden mit PALES jeweils für den ersten Eintrag der NMR-Struktur (1D3Z) berechnet.

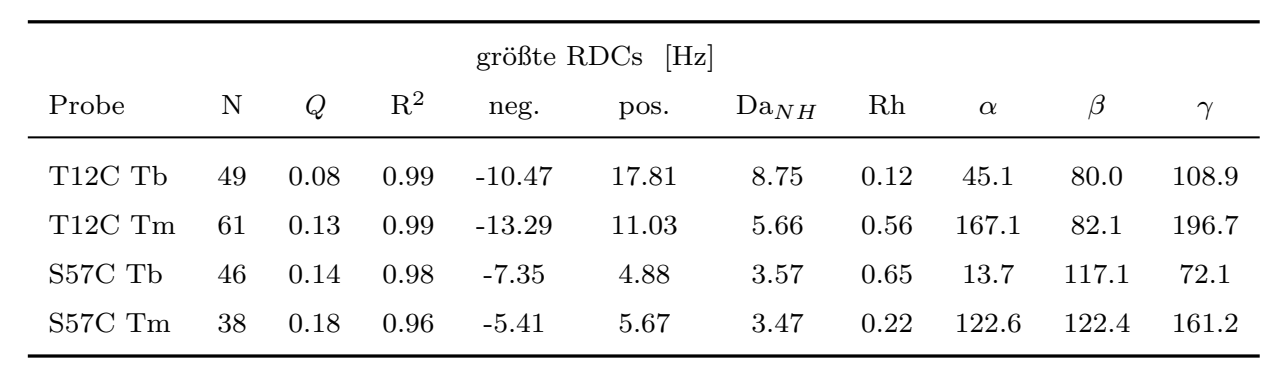

S57C legt diesen Schluss ebenfalls nahe. Die Ergebnisse der RDC-Analysen sind in Tabelle 5.3 zusammengefasst. Die RDCs von S57C Tb und S57C Tm weisen dennoch $Q$-Faktoren von unter 0.20 bei Korrelationsfaktoren $\geq 0.96$ auf, was auch die Qualität dieser Daten zeigt. Herauszuheben ist jedoch T12C Tb, da mit dieser Probe viele große RDCs von bis zu $17.8 \mathrm{~Hz}$ bei einem äußerst niedrigen $Q$-Faktor erhalten wurden. Die gemessenen RDCs waren demnach etwa doppelt so groß, wie die der EDTA-basierten Tags (Haberz et al. (2006)). Die vollständigen RDCs aller Proben finden sich im Anhang in den Tabellen 7.11 und 7.12. 

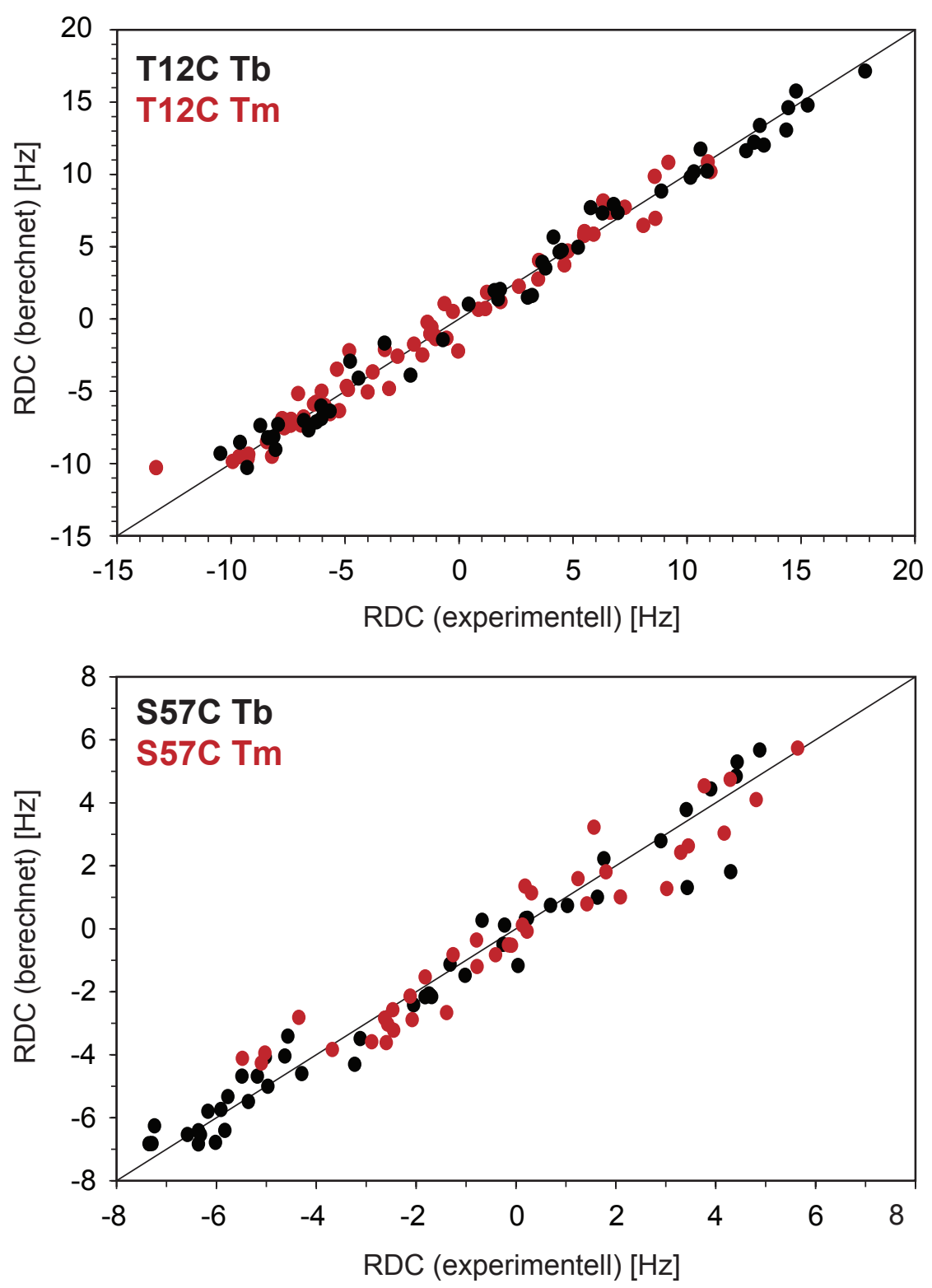

Abb. 5.16: Auftragung der experimentellen RDCs beider Cys-Ph-TAHA getaggten Ubiquitinmutanten (T12C: oben, S57C: unten) gegen die berechneten RDCs. Die Werte für Terbium sind schwarz, die für Thulium rot dargestellt. Die zu Grunde liegenden Kopplungskonstanten und RDCs sind in den Tabellen 7.11 und $7.12 \mathrm{im}$ Anhang angegeben. 


\subsection{Vergleich der PCS- und RDC-Tensoren}

Um die mit Cys-Ph-TAHA erhaltenen RDC- und PCS-Tensoren miteinander vergleichen zu können, wurden die RDCs ebenfalls mit dem Skript von Dr. Mitcheell Maestre Martinez berechnet. Für die einzelnen Strukturen waren die Ergebnisse identisch mit den von PALES berechneten. Für die weiteren Untersuchungen wurde allerdings, analog zur PCS-Auswertung, jeweils ein gemittelter Tensor aus allen zehn Strukturen (1D3Z) verwendet.

Grundsätzlich sollten die aus den RDC- und PCS-Analysen berechneten Tensoren einander gleich sein. Es zeigte sich in der Vergangenheit jedoch, dass die erhaltenen Tensoren zum Teil deutliche Unterschiede aufweisen. Die RDC-abgeleiteten Tensoren sind in der Regel kleiner, als die der PCSs. Dies wurde sowohl für die EDTA-basierten Tags, als auch für CLaNP-5 (Keizers et al. (2008)) und DOTA-M8 (Häussinger et al. (2009)) berichtet. Der Grund hierfür liegt in der unterschiedlichen Sensitivität der PCSs und RDCs auf Mittelung durch Bewegung. Da CLaNP-5 über zwei Disulfidbrücken am Protein verankert wird, sind dort die Differenzen etwas geringer, als bei einfach gebundenen Tags, dennoch sind die PCS-Tensoren etwa 20\% größer. Um den Einfluss der intrinsischen Proteinbewegungen zu reduzieren, wurden die RDC-Rechnungen zusätzlich mit dem EROS-Ensemble (Lange et al. (2008)) an Stelle von 1D3Z durchgeführt. Dieses Ensemble beschreibt den konformationellen Raum von Ubiquitin. Im Durchschnitt vergrößerten sich die axialen Tensorkomponenten der vier Proben um etwa 10\%. Die verbleibende Differenz beruht vermutlich auf von der Proteindynamik entkoppelten Bewegungen des Tags.

Die Ergebnisse der Berechnungen sind in Tabelle 5.4 zusammengefasst. Auffällig waren die unterschiedlichen Vorzeichen der axialen Komponenten $\Delta \chi_{a x}$ in drei der vier Proben. Wie den Darstellungen der Tensoren zu entnehmen ist (siehe Abbildung 7.4-7.7 im Anhang), weisen die positiven und negativen Bereiche der Tensoren in der Regel jedoch ähnliche Orientierungen auf. Die unterschiedlichen Vorzeichen beruhen somit auf den Definitionen von $\Delta \chi_{a x}$ und $\Delta \chi_{r h}$. Die PCS- und RDC-abgeleiteten Tensoren von $\mathrm{T} 12 \mathrm{C} \mathrm{Tb}$ hingegen weisen stärkere Abweichungen voneinander auf (siehe Abbildung 5.17). 

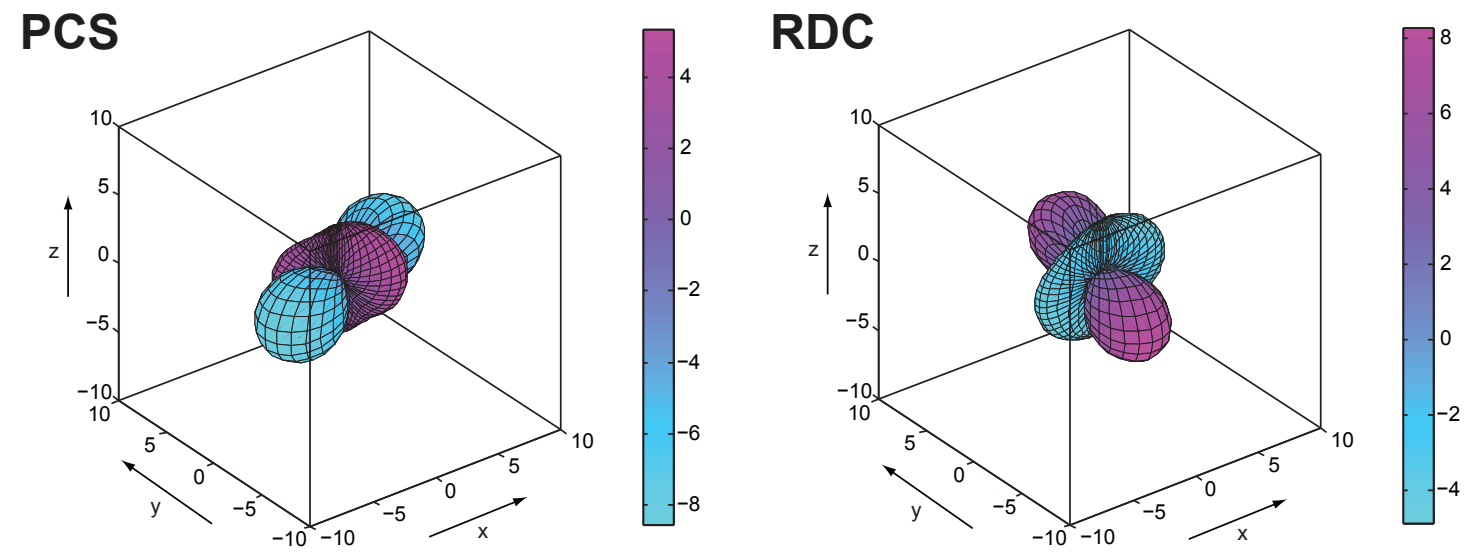

Abb. 5.17: T12C Tb: Vergleich der PCS- und RDC-abgeleiteten Tensoren $\left[10^{-4}\right]$. Diese weisen unterschiedliche Orientierungen und Vorzeichen auf. Die jeweiligen Elemente der Saupematrix sind im Anhang in der Tabelle 7.16 angegeben.

Tab. 5.4: Übersicht über die berechneten Tensoren aus den PCSs und RDCs der vier anisotropen Proben. Gegeben sind die axialen und rhombischen Komponenten des jeweiligen $\chi$-Tensors $\Delta \chi_{a x}$ und $\Delta \chi_{r h}\left[10^{-32} \mathrm{~m}^{3}\right]$, sowie die Eulerwinkel $\alpha$, $\beta$ und $\gamma\left[^{\circ}\right]$. Die Daten wurden auf der Grundlage der 1D3Z-NMR-Strukturen sowie des EROS-Ensembles berechnet $\left[10^{-32} \mathrm{~m}^{3}\right]$. Zusätzlich sind die jeweiligen $Q$-Faktoren und Korrelationsfaktoren $\mathrm{R}$ gegeben.

\begin{tabular}{lccccccccc}
\hline Probe & \multicolumn{2}{c}{ Daten } & $\Delta \chi_{a x}$ & $\Delta \chi_{r h}$ & $\alpha$ & $\beta$ & $\gamma$ & $Q$ & $\mathrm{R}^{2}$ \\
\hline \multirow{2}{*}{ T12C Tb } & PCS & 1D3Z & -14.68 & -1.82 & 141.34 & 90.73 & -22.89 & 0.19 & 0.97 \\
& RDC & 1D3Z & 14.38 & 1.64 & 133.98 & 94.15 & -74.36 & 0.12 & 0.99 \\
& RDC & EROS & 15.59 & 1.47 & - & - & - & 0.09 & 0.99 \\
\hline \multirow{2}{*}{ T12C Tm } & PCS & 1D3Z & -13.17 & -3.99 & 5.63 & 72.33 & 100.30 & 0.15 & 0.98 \\
& RDC & 1 D3Z & 9.27 & 5.34 & 6.84 & 98.28 & 12.90 & 0.13 & 0.99 \\
& RDC & EROS & 10.29 & 5.44 & - & - & - & 0.15 & 0.98 \\
\hline \multirow{2}{*}{ S57C Tb } & PCS & 1D3Z & -12.66 & -8.25 & -19.92 & 82.18 & -21.96 & 0.07 & 0.99 \\
& RDC & 1D3Z & 5.91 & 3.75 & -10.39 & 59.00 & -112.54 & 0.17 & 0.97 \\
& RDC & EROS & 6.49 & 4.14 & - & - & - & 0.14 & 0.98 \\
\hline \multirow{2}{*}{ S57C Tm } & PCS & 1D3Z & 8.44 & 1.21 & -139.51 & 47.95 & -12.45 & 0.11 & 0.98 \\
& RDC & 1D3Z & 5.69 & 1.23 & -133.94 & 54.18 & -18.05 & 0.26 & 0.93 \\
& RDC & EROS & 6.81 & 1.66 & - & - & - & 0.30 & 0.91 \\
\hline
\end{tabular}


Für die deutlichen Abweichungen der RDC- und PCS-Tensoren voneinander gibt es mehrere mögliche Erklärungen. Zum einen nehmen die NH-Vektoren im Protein unterschiedliche Orientierungen ein. Dadurch wird der dreidimensionale Raum gut erfasst, was in einer zuverlässigen Beschreibung des RDC-Tensors resultiert. Im Falle des Alignments durch paramagnetische Tags liegt das Metallion außerhalb des Proteins und die Metall-Kern-Vektoren werden nur auf einem kleinen Teil der Kugeloberfläche rund um das Metall erhalten. Dies kann zu einer gewissen Unsicherheit des PCS-Tensors führen. Dies ist in Abbildung 5.18 schematisch dargestellt.

Eine weitere potentielle Ursache für die unterschiedlichen Tensoren liegt in der Tensorberechnung selbst, da gleichzeitig auch noch die Metallposition bestimmt wird. Während der Minimierung können Verletzungen des Tensors durch leichte Veränderung der Position kompensiert werden. Wenn, wie im Fall von S57C Tb und S57C Tm, alle PCSs das gleiche Vorzeichen haben, ist dies besonders einfach möglich. Dies trägt dazu bei, dass für beide Proben die $Q$-Faktoren deutlich niedriger, als die der RDCs waren (siehe Tabelle 5.1).

Obwohl die Tensoren, wie in Tabelle 5.4 und Abbildung 5.17 gezeigt verschieden waren, konnten die PCSs im jeweiligen RDC-Tensor zurück berechnet werden. Diese Werte wurden in einem Korrelationsdiagramm gegen die experimentellen PCSs auf-

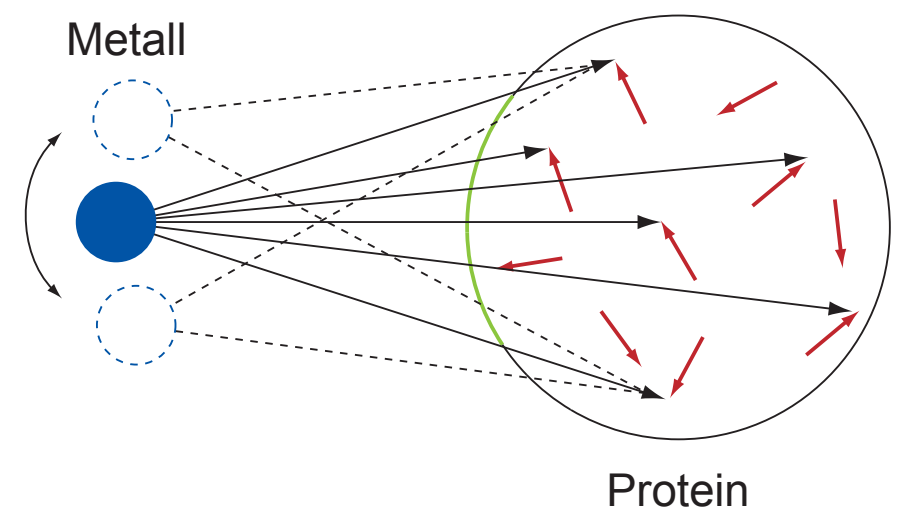

Abb. 5.18: Schematischer Vergleich zwischen PCSs und RDCs. Die Interspinvektoren (rot) beschreiben im Protein alle möglichen Raumrichtungen, wodurch der RDC-Tensor zuverlässig wird. Dagegen erfassen die Metall-Kern-Vektoren (schwarz) bei Tag-gebundenen Metallionen nur einen kleinen Winkelbereich der Kugeloberfläche (grün), wodurch sie fehleranfällig werden. 
Tab. 5.5: Ergebnisse der Kreuzberechnungen. Aufgeführt sind jeweils $Q$-Faktor und Korrelationsfaktor der Berechnungen der PCSs im RDC-Tensor, sowie der Berechnung der RDCs im PCS-Tensor.

\begin{tabular}{lllll}
\hline & \multicolumn{2}{c}{ PCSs im RDC-Tensor } & \multicolumn{2}{c}{ RDCs im } \\
Probe & $Q$ & $\mathrm{R}^{2}$ & $Q$ & $\mathrm{R}^{2}$ \\
\hline $\mathrm{T} 12 \mathrm{C} \mathrm{Tb}$ & 0.32 & 0.90 & 1.43 & 0.00 \\
$\mathrm{~T} 12 \mathrm{C} \mathrm{Tm}$ & 0.24 & 0.95 & 0.63 & 0.84 \\
S57C Tb & 0.28 & 0.92 & 1.05 & 0.84 \\
S57C Tm & 0.32 & 0.87 & 0.62 & 0.89 \\
\hline
\end{tabular}

getragen (siehe Abbildung 5.19). In weiten Teilen stimmen die, im RDC-Tensor berechneten PCSs mit den experimentellen gut überein, was sich auch in den $Q$-Faktoren von 0.24-0.32 ausdrückt (siehe Tabelle 5.5). Für S57C Tb und S57C Tm sind die berechneten Werte tendenziell unterschätzt und somit kleiner, als die experimentellen. Dies erklärt sich durch die deutlich geringere Größe der RDC-Tensoren im Vergleich zu den PCS-Tensoren. Für T12C Tb und T12C Tm zeigen die Datenpunkte eine Streuung um die Korrelationsgerade. Im Falle von T12C Tm waren der PCSund der RDC-Tensor einander sehr ähnlich, weshalb sich die PCSs im RDC-Tensor zurück berechnen lassen. Bei T12C Tb hingegen wurde die gute Korrelation durch eine Verschiebung der Metallposition im Zuge der Minimierung erreicht. Eine Änderung der Metallposition um wenige Ångström kann zu einem Vorzeichenwechsel des PCS-Tensors führen, ohne dass die zurück berechneten PCSs große Abweichungen von den experimentellen Werten aufweisen.

Zum Vergleich wurde das umgekehrte Experiment durchgeführt und die RDCs im PCS-Tensor zurück berechnet. Die Ergebnisse sind in Abbildung 5.20 gegen die experimentellen Werte aufgetragen. Es ist zu sehen, dass die experimentellen Werte deutliche Unterschiede zu den im PCS-Tensor zurück berechneten RDCs aufweisen. Im Fall von T12C Tb besteht überhaupt keine Korrelation der Daten mehr, da die unterschiedlichen Orientierungen der PCS- und RDC-Tensoren bei der Zurückberechnung der RDCs im PCS-Tensor nicht kompensiert werden können. Für die anderen Proben sind die experimentellen Werte erwartungsgemäß überschätzt. 

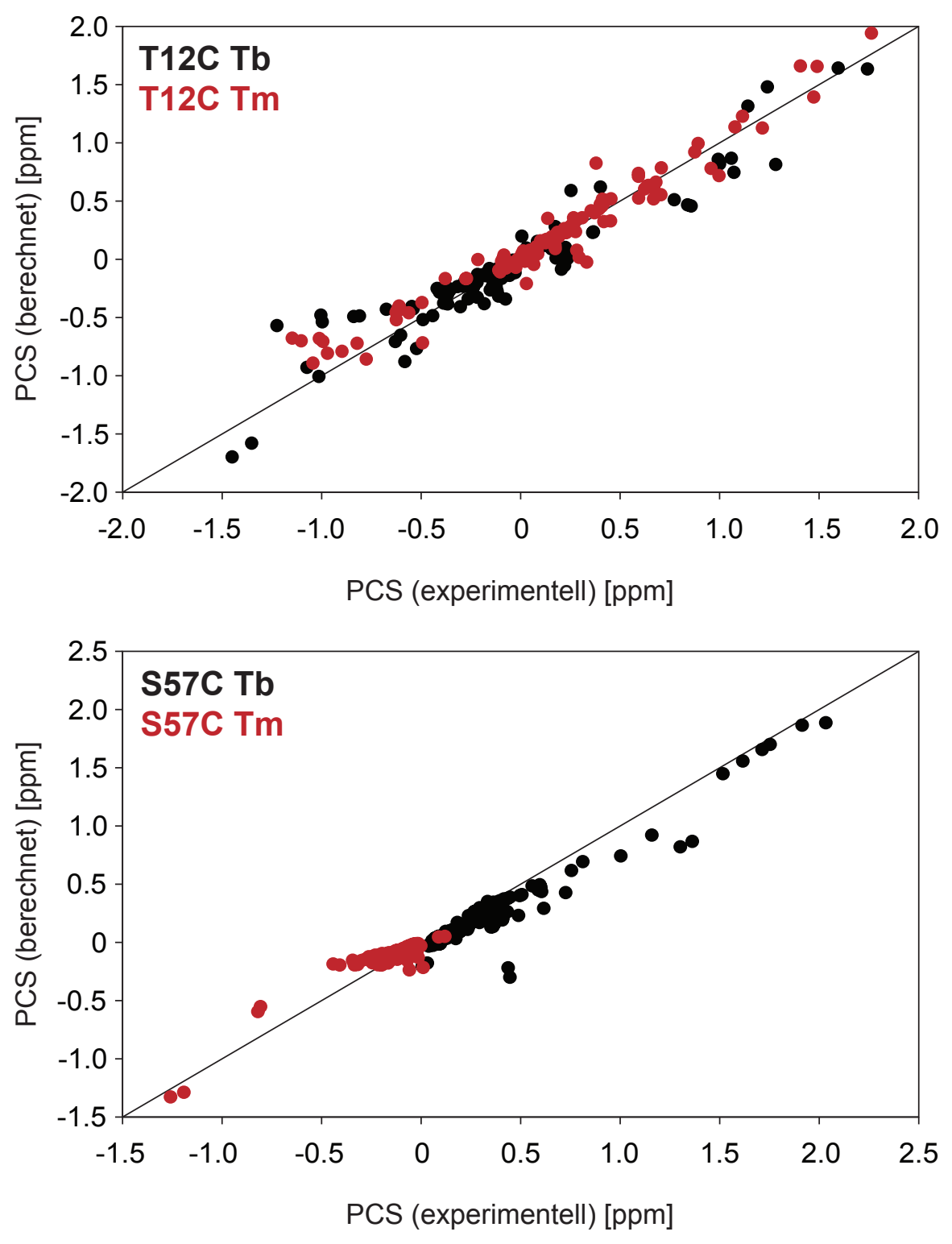

Abb. 5.19: Korrelationsdiagramm der experimentellen ${ }^{1} \mathrm{H}-$ und ${ }^{15} \mathrm{~N}-\mathrm{PCSs}$ beider CysPh-TAHA-getaggten Ubiquitinmutanten (T12C: oben, S57C: unten), aufgetragen gegen die im RDC-Tensor zurück berechneten Werte. Die Daten für Terbium sind schwarz, die für Thulium rot dargestellt. Die zu Grunde liegenden Daten sind im Anhang in den Tabellen 7.13 und 7.14 gegeben. 

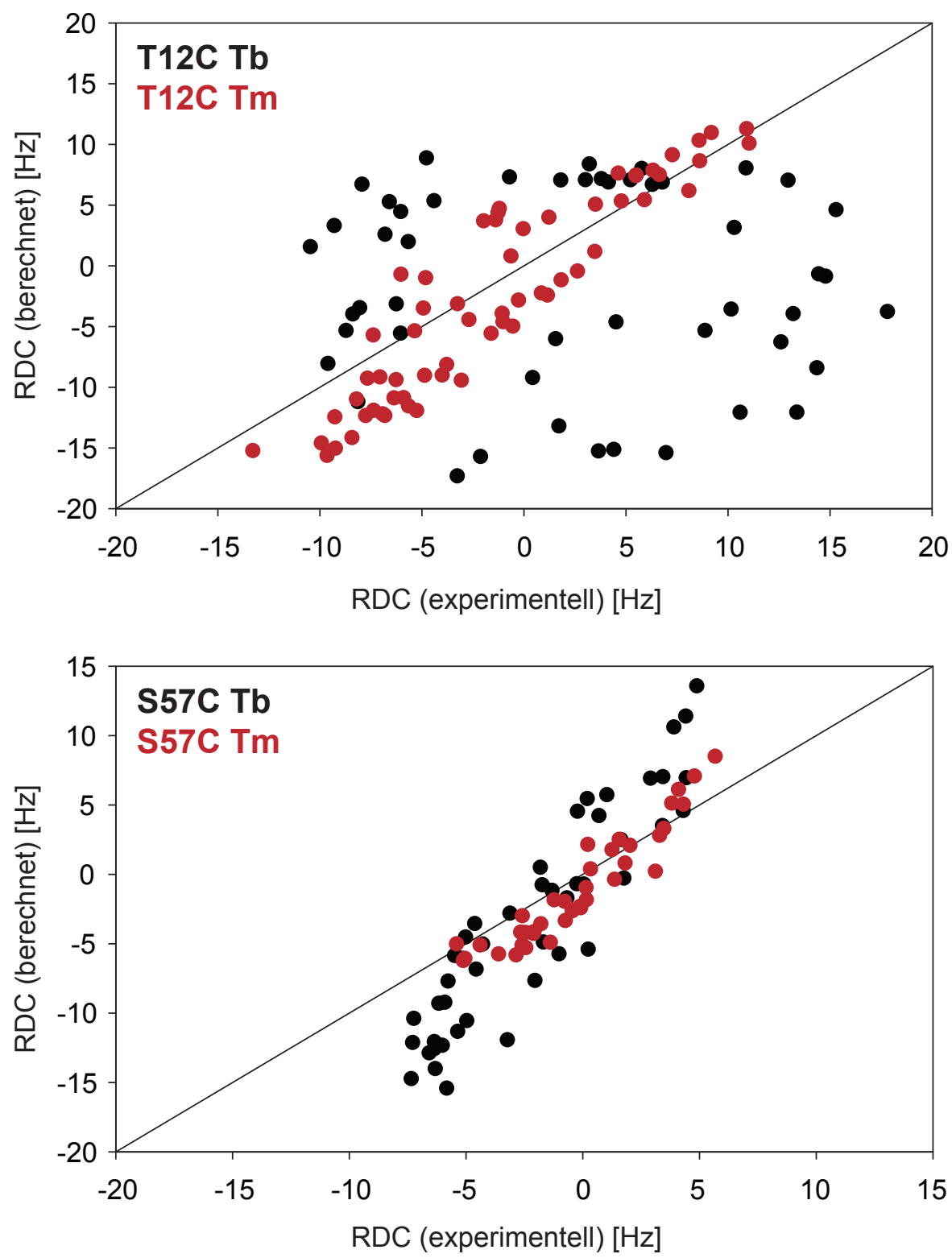

Abb. 5.20: Korrelationsdiagramm der experimentellen RDCs beider Cys-Ph-TAHAgetaggten Ubiquitinmutanten (T12C: oben, S57C: unten), aufgetragen gegen die im PCS-Tensor zurück berechneten Werte. Die Daten für Terbium sind schwarz, die für Thulium rot dargestellt. Die zu Grunde liegenden Daten sind im Anhang in den Tabellen 7.11, 7.12 und 7.15 gegeben. 
Ein Problem bei der Minimierung der PCSs und der Metallposition liegt darin, dass die Energiehyperfläche kein ausgeprägtes Minimum aufzuweisen scheint. Abweichungen von den experimentellen PCSs können dann ohne schwerwiegende Auswirkungen auf den $Q$-Faktor oder den Korrelationsfaktor durch leichte Verschiebungen der Metallpositon kompensiert werden. Um dies zu unterbinden, sollte der PCS-Tensor von RDC-Daten unterstützt berechnet werden. Es wurden zwei Verfahren getestet. In dem ersten Ansatz wurden die RDCs und PCSs gleichzeitig minimiert und somit ein gemeinsamer Tensor bestimmt. Bei dem zweiten Ansatz wurde, basierend auf dem RDC-abgeleiteten Tensor, die Position des Metalls berechnet und anschließend die PCSs um diese Position herum minimiert. Die Ergebnisse aller Berechnungen sind in der Tabelle 5.6 zusammengefasst. Die Abweichungen der PCS-Tensoren (A) im Vergleich zu den in Tabelle 5.1 gegebenen beruhen darauf, dass die Reste R74-G76 für die finalen Berechnungen, wie auch für die RDC-Berechnungen (B), ausgeschlossen wurden. 
Tab. 5.6: Übersicht über die erhaltenen Tensoren. Gegeben sind die berechneten Metallkoordinaten im Molekülkoordinatensystem $[\AA], \Delta \chi_{a x}$ und $\Delta \chi_{r h}\left[10^{-32} \mathrm{~m}^{3}\right]$ und die Eulerwinkel $\left[^{\circ}\right]$. Es sind die gemittelten Tensoren aller Strukturen (1D3Z) gezeigt. Die Tensoren sind wie folgt zugeordnet: PCS-Tensor (A); RDC-Tensor (B); PCSs im RDC-Tensor (C); PCSs und RDCs zusammen minimiert: PCSs (D), RDCs (E); Metallposition bestimmt auf der Basis des RDC-Tensors mit anschließender Minimierung der PCSs (F).

\begin{tabular}{|c|c|c|c|c|c|c|c|c|c|c|c|c|}
\hline Probe & & aten & $Q$ & $\mathrm{R}^{2}$ & $\mathrm{x}$ & $\mathrm{y}$ & $\mathrm{z}$ & $\Delta \chi_{a x}$ & $\Delta \chi_{r h}$ & $\alpha$ & $\beta$ & $\gamma$ \\
\hline \multirow[t]{6}{*}{$\mathrm{T} 12 \mathrm{C} \mathrm{Tb}$} & $\mathbf{A}$ & 1D3Z & 0.18 & 0.97 & 35.75 & -77.04 & 5.01 & -14.83 & -2.51 & 141.82 & 92.73 & -22.96 \\
\hline & B & 1D3Z & 0.12 & 0.99 & - & - & - & 14.38 & 1.64 & 133.98 & 94.15 & -74.36 \\
\hline & C & 1D3Z & 0.32 & 0.90 & 38.27 & -68.83 & 1.00 & 14.38 & 1.64 & 133.98 & 94.15 & -74.36 \\
\hline & D & 1D3Z & 0.28 & 0.92 & 38.31 & -69.41 & 1.68 & 14.46 & 3.34 & 135.56 & 95.70 & -74.10 \\
\hline & $\mathbf{E}$ & 1D3Z & 0.15 & 0.98 & 38.31 & -69.41 & 1.68 & 14.46 & 3.34 & 135.56 & 95.70 & -74.10 \\
\hline & $\mathbf{F}$ & 1D3Z & 0.26 & 0.93 & 38.27 & -68.83 & 1.00 & 16.76 & 6.76 & 131.73 & 89.73 & -71.32 \\
\hline \multirow[t]{6}{*}{$\mathrm{T} 12 \mathrm{C} \mathrm{Tm}$} & $\mathbf{A}$ & 1D3Z & 0.15 & 0.98 & 38.18 & -71.92 & 6.27 & -13.42 & -3.84 & 7.22 & 72.81 & 100.82 \\
\hline & B & 1D3Z & 0.18 & 0.97 & - & - & - & 9.27 & 5.34 & 6.84 & 98.28 & 12.90 \\
\hline & $\mathrm{C}$ & 1D3Z & 0.24 & 0.95 & 39.53 & -71.68 & 5.37 & 9.27 & 5.34 & 6.84 & 98.28 & 12.90 \\
\hline & D & 1D3Z & 0.20 & 0.96 & 39.14 & -72.08 & 5.08 & -8.99 & -5.87 & 9.76 & 81.04 & 100.09 \\
\hline & $\mathbf{E}$ & 1D3Z & 0.20 & 0.96 & 39.14 & -72.08 & 5.08 & -8.99 & -5.87 & 9.76 & 81.04 & 100.09 \\
\hline & $\mathbf{F}$ & 1D3Z & 0.16 & 0.97 & 39.53 & -71.68 & 5.37 & -11.28 & -2.84 & 15.24 & 75.51 & 106.17 \\
\hline \multirow[t]{6}{*}{$\mathrm{S} 57 \mathrm{C} \mathrm{Tb}$} & $\mathbf{A}$ & 1D3Z & 0.07 & 0.99 & 59.72 & -91.74 & 12.04 & -12.71 & -8.28 & 19.42 & 98.09 & 158.25 \\
\hline & B & 1D3Z & 0.17 & 0.96 & - & - & - & 5.91 & 3.75 & -10.39 & 59.00 & -112.54 \\
\hline & C & 1D3Z & 0.27 & 0.92 & 57.41 & -88.62 & 10.62 & 5.91 & 3.75 & -10.39 & 59.00 & -112.54 \\
\hline & D & 1D3Z & 0.19 & 0.96 & 57.52 & -88.99 & 10.92 & 6.75 & 3.34 & -6.63 & 57.39 & -113.30 \\
\hline & $\mathbf{E}$ & 1D3Z & 0.19 & 0.96 & 57.52 & -88.99 & 10.92 & 6.75 & 3.34 & -6.63 & 57.39 & -113.30 \\
\hline & $\mathbf{F}$ & 1D3Z & 0.12 & 0.97 & 57.41 & -88.62 & 10.62 & 7.13 & 4.27 & 20.08 & 54.14 & -114.52 \\
\hline \multirow[t]{6}{*}{$\mathrm{S} 57 \mathrm{C} \mathrm{Tm}$} & $\mathbf{A}$ & 1D3Z & 0.11 & 0.98 & 59.73 & -86.67 & 11.07 & 8.47 & 1.22 & -139.37 & 47.76 & -12.71 \\
\hline & B & 1D3Z & 0.26 & 0.93 & - & - & - & 5.69 & 1.23 & -133.94 & 54.18 & -18.05 \\
\hline & C & 1D3Z & 0.33 & 0.84 & 60.07 & -83.71 & 11.68 & 5.69 & 1.23 & -133.94 & 54.18 & -18.05 \\
\hline & D & 1D3Z & 0.15 & 0.96 & 58.29 & -86.14 & 9.62 & 6.00 & 0.80 & -142.19 & 53.00 & -16.36 \\
\hline & $\mathbf{E}$ & 1D3Z & 0.27 & 0.93 & 58.29 & -86.14 & 9.62 & 6.00 & 0.80 & -142.19 & 53.00 & -16.36 \\
\hline & $\mathbf{F}$ & 1D3Z & 0.14 & 0.97 & 60.07 & -83.71 & 11.68 & 6.50 & 1.75 & -124.16 & 43.60 & 2.02 \\
\hline
\end{tabular}




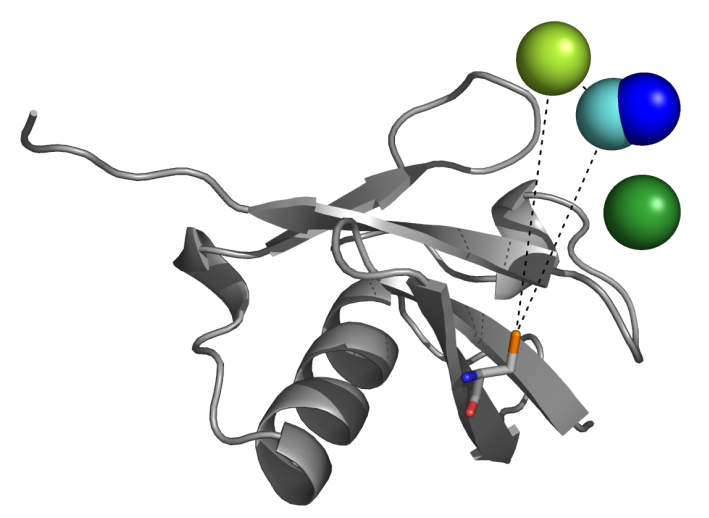

Abb. 5.21: Darstellung der Metallpositionen, berechnet aus den PCSs für T12C Tb (dunkelgrün) und T12C Tm (dunkelblau), sowie aus der gleichzeitigen Minimierung von PCSs und RDCs: T12C Tb (hellgrün), T12C Tm (hellblau).

Wie aus Tabelle 5.6 hervorgeht, lieferten für T12C Tb und T12C Tm sowohl die gleichzeitige Minimierung von RDCs und PCSs (D), als auch die PCS-Minimierung der RDC-abgeleiteten Position (F) beinahe identische Metallkoordinaten. In Abbildung 5.21 sind die Metallpositionen, die aus den PCS-Tensoren (A), beziehungsweise aus den Tensoren der gleichzeitigen Minimierung von PCSs und RDCs (D) erhalten wurden, zusammen mit der Ubiquitinstruktur gezeigt. Die entsprechende Darstellung für S57C befindet sich im Anhang (Abbildung 7.3). Während sich die Thuliumposition nur wenig ändert, ist die neue Terbiumlage $8.7 \AA$ von der aus (A) bestimmten Position entfernt, was den Wechsel des Vorzeichens erklärt. Dies wird auch durch Abbildung 5.22 illustriert. Die Vektorscharen (A) und (B) geben die Länge der Metall-Kern-Vektoren in Ångstrom wieder. Die Projektionen der jeweiligen Vektorscharen auf einer Kugeloberfläche (Abbildung 5.22 (C) und (D)) verdeutlichen, wie bereits in Abbildung 5.18 schematisch gezeigt, dass sich trotz einer Verschiebung der Metallposition von über $8 \AA$ die Verteilung der Metall-Kern-Vektoren auf der Kugeloberfläche relativ ähnlich bleibt. Die neue Lage wird in erster Linie durch Veränderungen der Tensorform und -orientierung kompensiert, ohne dass die zurück berechneten PCSs stark von den experimentellen Werten abweichen. So wurde für keine Pseudokontaktverschiebung $\geq \pm 0.1$ ppm ein anderes Vorzeichen, als das gemessene, zurück berechnet, obwohl den PCSs der RDC-Tensor zu Grunde liegt. 
A

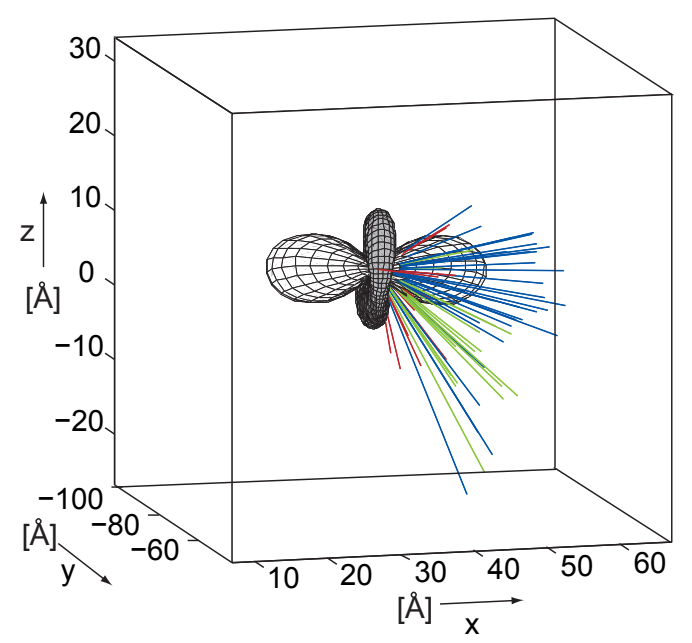

C

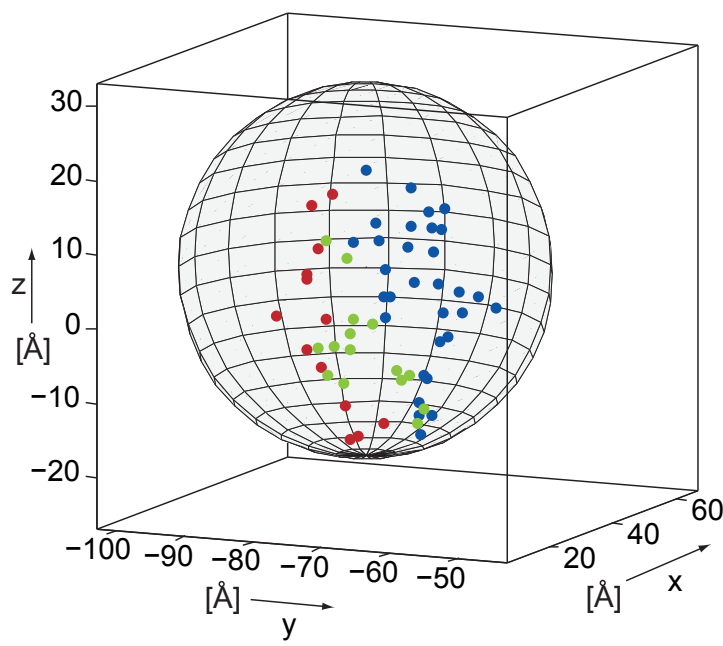

B

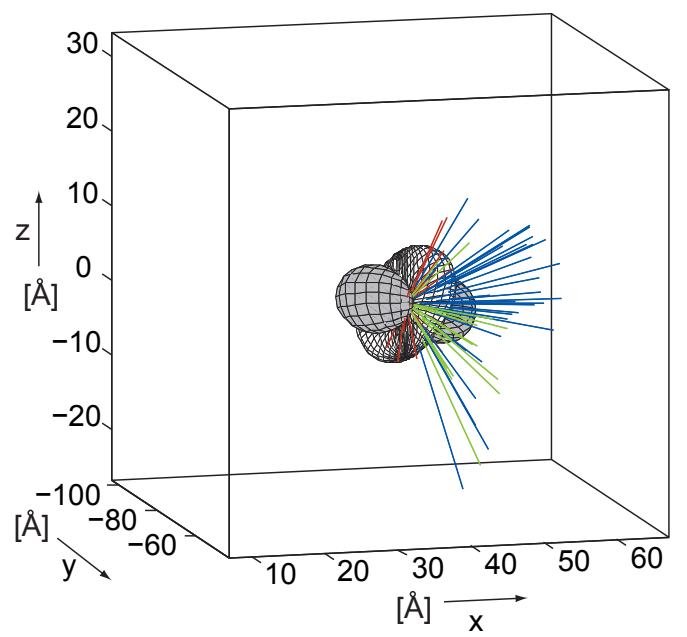

\section{D}

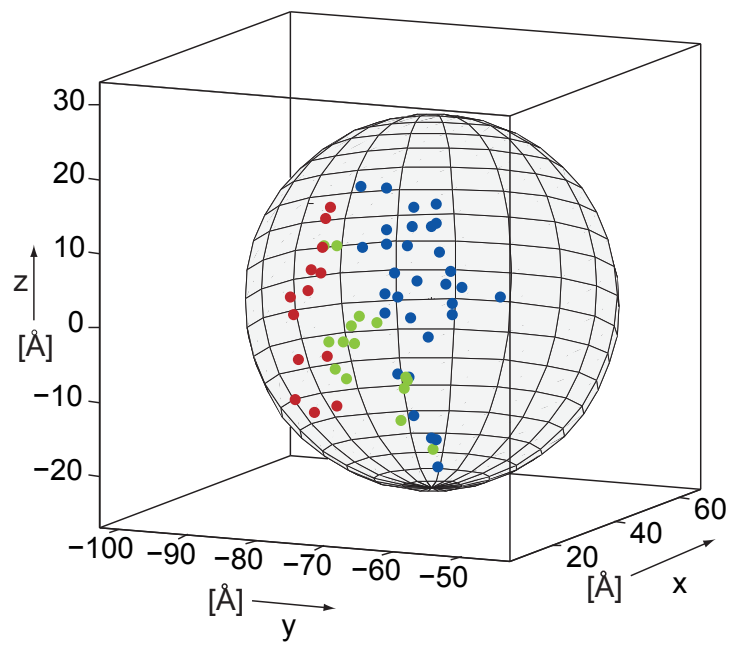

Abb. 5.22: T12C Tb: Darstellung der Metall-Kern-Vektoren der PCSs als Vektorschar $[\AA]$ für den PCS-Tensor (A). Die Tensoren sind nicht maßstabsgetreu abgebildet. Negative PCSs sind blau, positive rot und PCSs einer Größe von $0 \pm 0.1 \mathrm{ppm}$ grün dargestellt. (C) zeigt die Verteilungen der Vektorschar aus (A) auf der Kugeloberfläche, wobei der Kubus um $60^{\circ}$ gedreht wurde. (B) und (D) liegen die, im RDC-Tensor berechneten PCSs zu Grunde. Die unterschiedlichen zu Grunde liegenden Metallpositionen werden durch die Verschiebung der Sphären im Kubus angedeutet. 
In Abbildung 5.23 sind die, auf die beschriebene Weise neu berechneten PCS-Tensoren für beide anisotropen T12C-Proben im Vergleich zu den initialen RDC-Tensoren gezeigt. Die axialen Komponenten beider Proben weisen nun, durch die RDC-gestützte Berechnung, das selbe Vorzeichen auf.

Beide vorgestellten Methoden der PCS-Tensorberechnung liefern vergleichbare Metallpositionen. Wenn der PCS-Tensor auf Basis der RDC-bestimmten Lage mit anschließender Minimierung um diese Position bestimmt werden, so sind wie in Tabelle $5.6 \mathrm{zu}$ sehen, die Q-Faktoren und R-Werte der PCSs aller vier Proben erwartungsgemäß besser, als bei der gemeinsamen RDC- und PCS-Minimierung im selben Tensor. Die Differenz der Q-Faktoren zwischen den beiden Berechnungsmethoden beträgt für die PCSs zwischen 0.02 und 0.07. Es besteht hier allerdings die
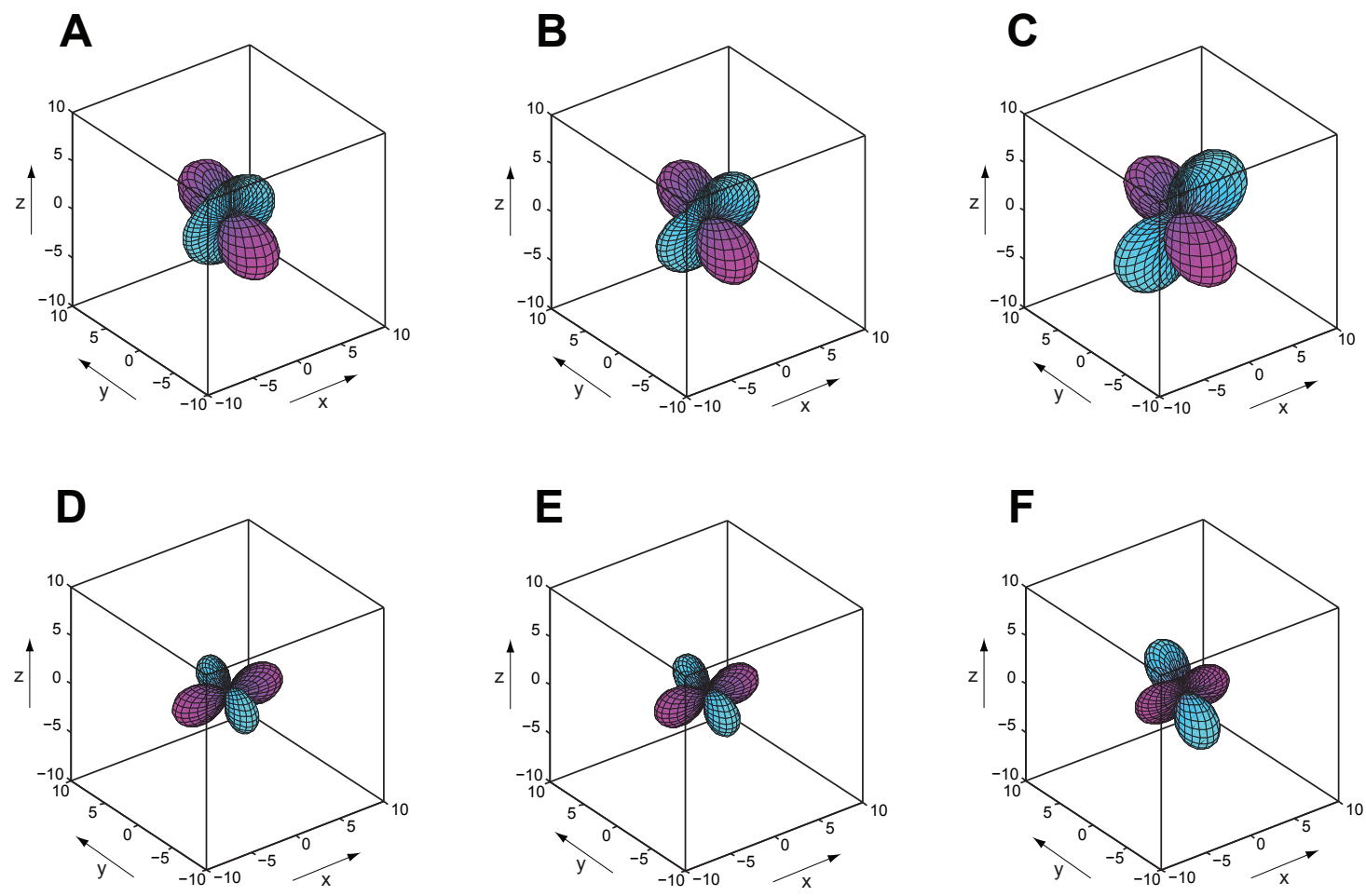

Abb. 5.23: Vergleich der RDC-Tensoren (A) und (D) mit den neu berechneten PCSTensoren. $(\mathrm{A})-(\mathrm{C})$ zeigen die Tensoren von $\mathrm{T} 12 \mathrm{C} \mathrm{Tb},(\mathrm{D})-(\mathrm{F})$ die von T12C Tm. (B) und (E) sind die PCS-Tensoren der gleichzeitigen Minimierung von RDCs und PCSs, (C) und (F) sind die PCS-Tensoren auf Basis der RDC-Tensoren mit anschließender PCS-Minimierung. Die Elemente der Saupematrix $\left[10^{-4}\right]$ sind im Anhang in der Tabelle 7.16 angegeben. 
Möglichkeit, dass sich im Zuge der Minimierung die Position des Metalls und somit auch der Tensor zurück in Richtung des PCS-Tensors bewegt. Dies zeigt sich daran, dass die so erhaltenen Tensoren wieder einen größeren Unterschied in der Orientierung im Vergleich zu den RDC-Tensoren aufweisen (siehe Tabelle 5.6). Die RDCs hingegen werden durch die gleichzeitige Minimierung von RDCs und PCSs nur sehr gering beeinflusst und werden durch den gemeinsamen Tensor sehr gut beschrieben. Die $Q$-Faktoren sind zwischen 0.01 und 0.03 höher, als die der unabhängigen Berechnungen der RDC-Tensoren. Somit scheint die Berechnung eines gemeinsamen Tensors, basierend auf PCS- und RDC-Daten, ein guter Ansatz zu sein, um PCSs und RDCs gleichzeitig zu betrachten.

In der Abbildung 5.24 sind die Korrelationsdiagramme der experimentellen ${ }^{1} \mathrm{H}$ - und ${ }^{15}$ N-PCSs gegen die, im gemeinsamen Tensor zurück berechneten Werte gezeigt. Die Daten von T12C Tb und T12C Tm streuen etwas stärker um die Korrelationsgerade, als die Werte von S57C Tb und S57C Tm, zeigen aber im Gegensatz zu diesen keine Tendenz auf. Beide S57C-Datensätze werden noch immer ein wenig unterschätzt, die Ausmaße sind jedoch geringer als bei der Berechnung der PCSs im RDC-Tensor (siehe Abbildung 5.19). Abbildung 5.25 zeigt die entsprechende Auftragung der RDCs. Für die S57C-Proben weichen die berechneten Werte auch stärker von den experimentellen ab, als für T12C. Dies hat vermutlich mehrere Ursachen. Zum einen waren die gemessenen RDCs bei S57C durch eine erhöhte Flexibilität des Tags deutlich kleiner, wodurch eventuelle Fehler stärker ins Gewicht fallen. Zum anderen wiesen alle PCSs in den jeweiligen Proben das selbe Vorzeichen auf. Für eine eindeutige Tensorbeschreibung ist jedoch ein Vorzeichenwechsel von besonderer Bedeutung. 

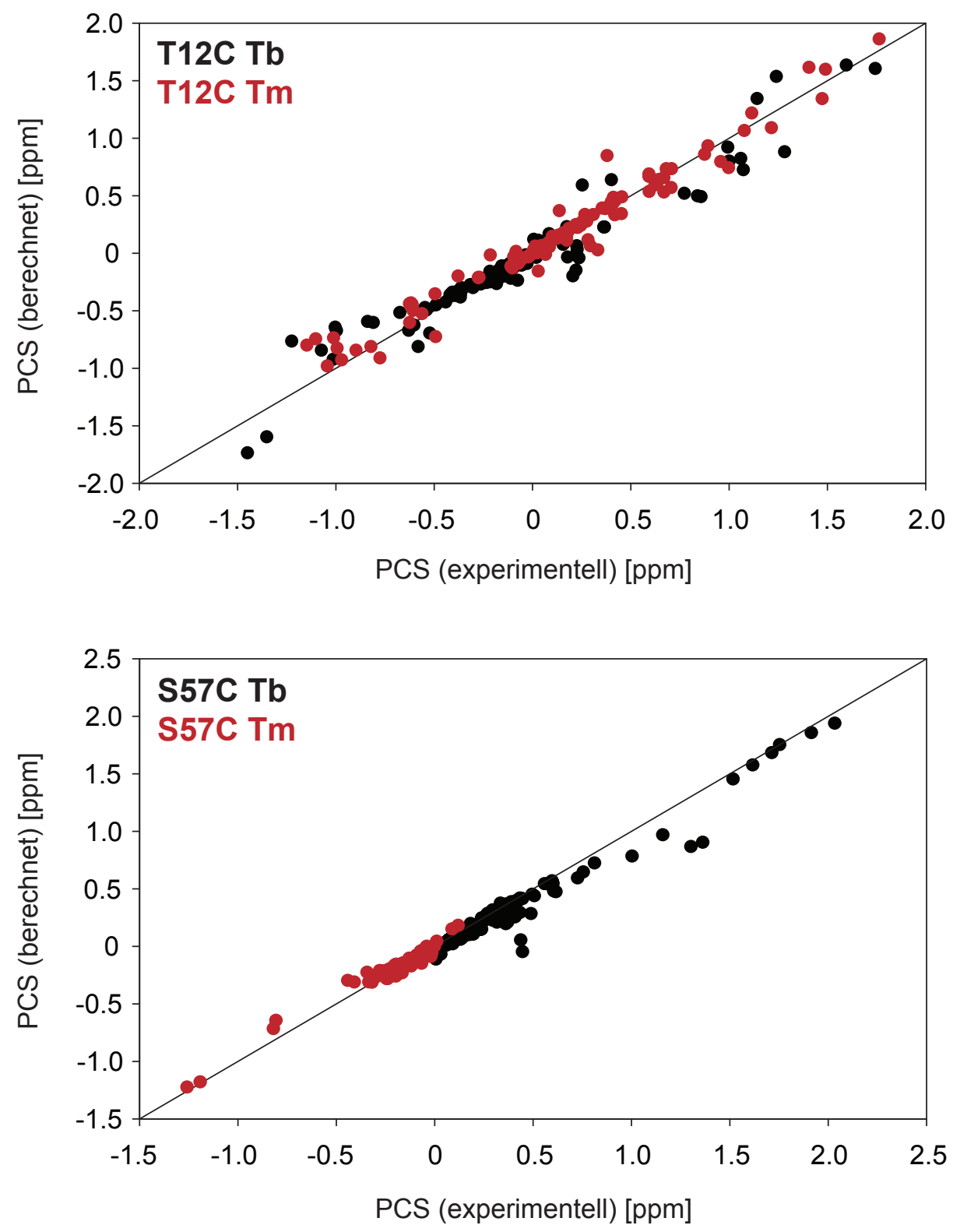

Abb. 5.24: Korrelationsdiagramm der experimentellen ${ }^{1} \mathrm{H}-$ und ${ }^{15} \mathrm{~N}-\mathrm{PCSs}$ beider CysPh-TAHA-getaggten Ubiquitinmutanten (T12C: oben, S57C: unten), aufgetragen gegen die mit den RDCs zusammen berechneten Werte. Die Daten für Terbium sind schwarz, die für Thulium rot dargestellt. Die zu Grunde liegenden Daten sind im Anhang in den Tabellen 7.17 und 7.18 gegeben. 

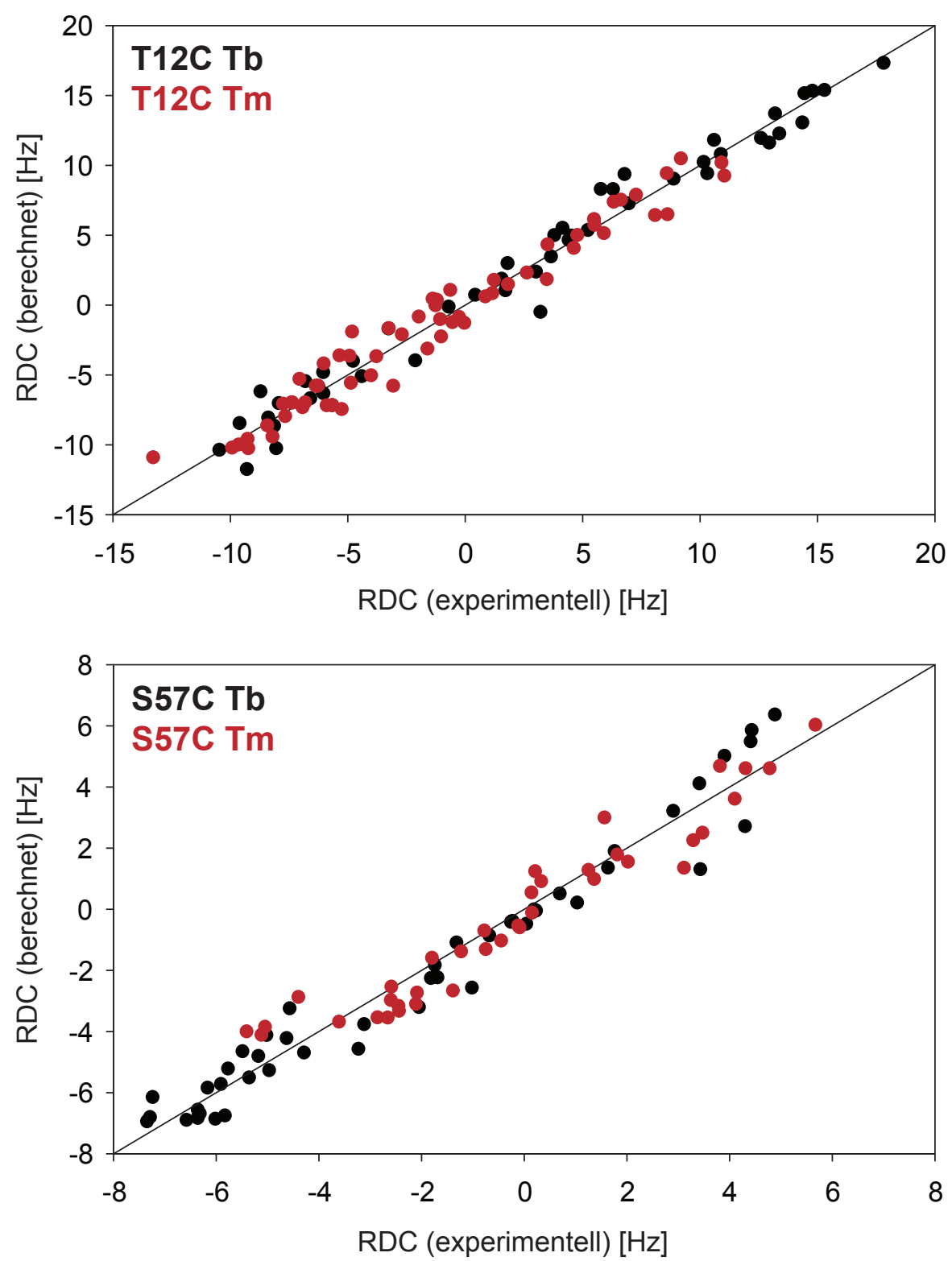

Abb. 5.25: Korrelationsdiagramm der experimentellen RDCs beider Cys-Ph-TAHAgetaggten Ubiquitinmutanten (T12C: oben, S57C: unten), aufgetragen gegen die mit den PCSs zusammen berechneten Werte. Die Daten für Terbium sind schwarz, die für Thulium rot dargestellt. Die zu Grunde liegenden Daten sind im Anhang in den Tabellen 7.11, 7.12 und 7.19 gegeben. 
Tab. 5.7: Übersicht über die Mittelwerte und Standardabweichungen der Saupematrixelemente von $\mathrm{T} 12 \mathrm{C} \mathrm{Tb}$ und $\mathrm{T} 12 \mathrm{C} \mathrm{Tm}$, die aus 100 Berechnungen mit jeweils $80 \%$ der Daten erhalten wurden $\left[10^{-5}\right]$.

\begin{tabular}{ccccccc}
\hline Probe & Daten & $\mathrm{S}_{z z}$ & $\mathrm{~S}_{x x-y y}$ & $\mathrm{~S}_{x y}$ & $\mathrm{~S}_{x z}$ & $\mathrm{~S}_{y z}$ \\
\hline \multirow{2}{*}{$\mathrm{T} 12 \mathrm{C} \mathrm{Tb}$} & $\mathrm{PCS}$ & $39.2 \pm 4.9$ & $-91.8 \pm 13.2$ & $-45.2 \pm 5.4$ & $0.0 \pm 4.9$ & $-9.4 \pm 7.2$ \\
& $\mathrm{RDC}$ & $-41.2 \pm 1.1$ & $-104.7 \pm 1.6$ & $32.7 \pm 0.8$ & $-4.5 \pm 0.8$ & $10.6 \pm 0.7$ \\
\hline \multirow{2}{*}{$\mathrm{T} 12 \mathrm{C} \mathrm{Tm}$} & PCS & $10.9 \pm 9.4$ & $111.1 \pm 12.4$ & $-21.2 \pm 8.9$ & $-3.0 \pm 6.2$ & $-28.2 \pm 7.0$ \\
& RDC & $-3.2 \pm 1.1$ & $91.7 \pm 1.5$ & $-21.2 \pm 0.7$ & $6.7 \pm 0.8$ & $-7.0 \pm 0.7$ \\
\hline
\end{tabular}

Um die Fehleranfälligkeit der Tensoren weiter zu untersuchen, wurden 100 Datensätze von Dr. Mitcheell Maestre Martinez erzeugt, aus denen zufällig jeweils $20 \%$ der Daten entfernt wurden. Mit diesen reduzierten Datensätzen wurden die jeweiligen PCS- und RDC-Tensoren erneut berechnet. Repräsentativ sind für T12C Tb und T12C Tm in Abbildung 5.26 drei der zehn möglichen Kombinationen der Saupematrixelemente aufgetragen. Während die RDC-Tensoren nur eine geringe Streuung zeigen, wird die große Fehleranfälligkeit der PCS-Tensoren direkt ersichtlich. Darüber hinaus ist gut zu erkennen, dass die Tensoren aus der gemeinsamen Minimierung von PCSs und RDCs den RDC-abgeleiteten Tensoren sehr ähnlich sind. Im Gegensatz dazu ergeben sich durch die PCS-Minimierung rund um die RDC-abgeleitete Metallposition wieder größere Unterschiede zum RDC-Tensor. In Tabelle 5.7 sind die Mittelwerte und Standardabweichungen der Matrixelemente aufgelistet.

Grundsätzlich ist für die Bestimmung eines Tensors eine möglichst hohe Anzahl an Messwerten erstrebenswert. Durch die, in der vorgestellten Methode, gleichzeitige Minimierung von RDCs und PCSs werden zur Tensorbeschreibung deutlich mehr Parameter verwendet, als bei den unabhängigen Berechnungen der jeweiligen Tensoren. Die RDCs haben bei der gemeinsamen Minimierung von RDCs und PCSs einen großen Einfluss auf den späteren Tensor. Somit wirken sich große RDCs besonders positiv auf die Berechnung aus. 

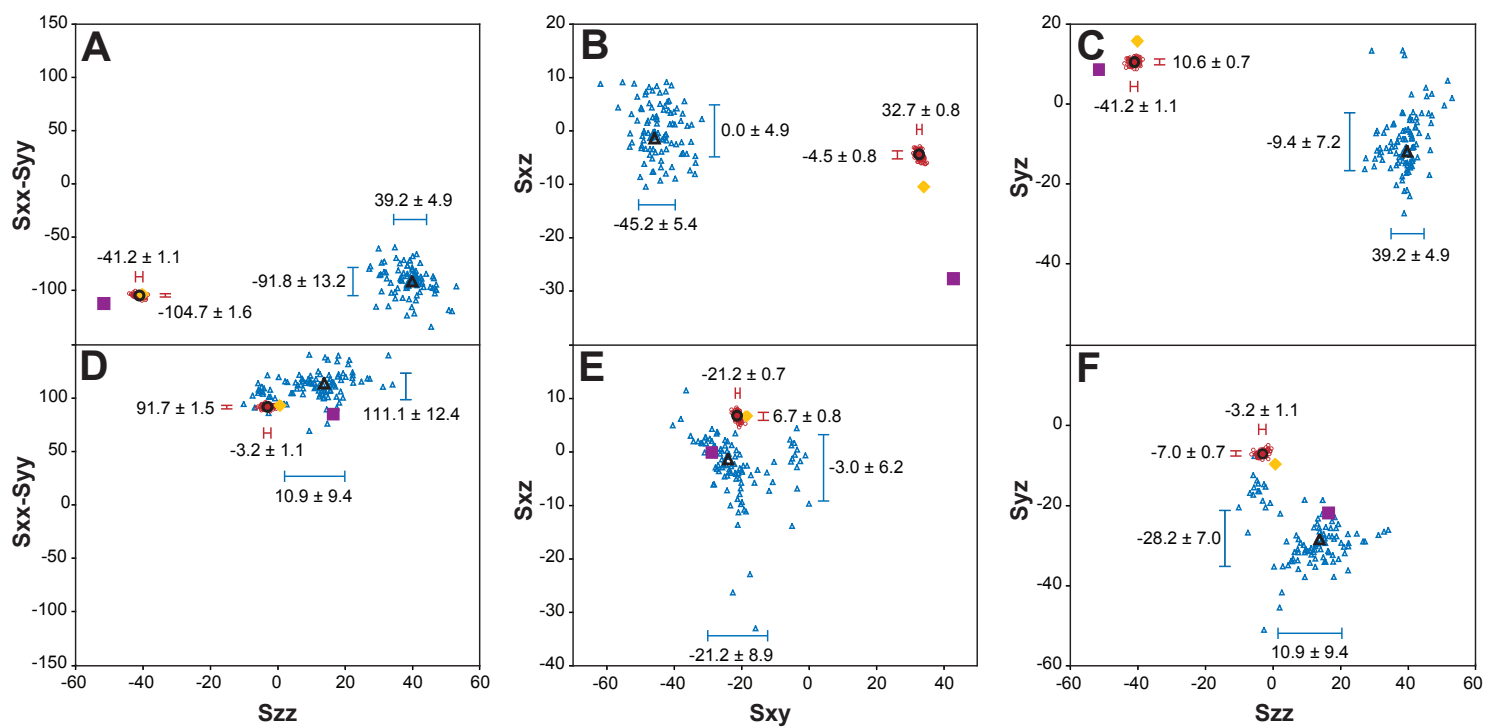

Abb. 5.26: Auftragungen der Saupematrixelemente $\left[10^{-5}\right]$ von $\mathrm{T} 12 \mathrm{C} \mathrm{Tb}(\mathrm{A}-\mathrm{C})$ und T12C Tm (D-F), die aus den unterschiedlichen Berechnungen erhalten wurden. Repräsentativ gezeigt sind jeweils drei der zehn möglichen Kombinationen. Die Daten sind wie folgt markiert: PCS-Tensor: schwarzes Dreieck, RDC-Tensor: schwarzer Kreis, Tensor der gemeinsamen Minimierung von PCSs und RDCs: gelbe Raute, Tensor der RDC-basierten Metallposition mit anschließender Minimierung der PCSs: violetter Kasten. Zusätzlich sind jeweils 100 Datenpunkte für die PCSs (blaue Dreiecke) und RDCs (rote Kreise) angegeben, bei deren Berechnung jeweils 20\% der Daten zufällig ausgelassen wurden. Die Balken geben die resultierenden Mittelwerte mit den entsprechenden Standardabweichungen an (siehe auch Tabelle 5.7). 


\subsection{Zusammenfassung und Einordnung der Ergeb- nisse}

Mit dem Cys-Ph-TAHA-Tag wurde ein viel versprechendes Werkzeug für die NMRSpektroskopie entwickelt. Cys-Ph-TAHA ist synthetisch in Maßstäben von mehreren hundert Milligramm leicht zugänglich. Der Tag ist chemisch sehr stabil und zeigte auch nach Monaten der Lagerung keine Dekompositionserscheinungen. Darüber hinaus wurde eine zuverlässige und gleichzeitig sehr einfach auszuführende Methode entwickelt, mit der Metall-vorbeladenes Cys-Ph-TAHA nahezu quantitativ an Proteine gebunden werden kann. Die erhaltenen Spektren waren von hervorragender Qualität, was sich in einer geringen Linienverbreiterung in Bezug auf die isotrope Referenz ausdrückte. Es wurden PCSs und vor allem RDCs von hoher Qualität erhalten. Die Daten zeigten zum einen eine sehr gute Korrelation mit den zurück berechneten Werten, zum anderen wurden große RDCs erhalten. Hervorzuheben ist die Probe T12C Tb, mit der RDCs von bis zu 17.8 Hz gemessen wurden. Im Vergleich zu den EDTA-basierten Tags entspricht das etwa einer Verdopplung der maximalen RDCs. Da der gleiche Linker, wie bei den EDTA-Tags verwendet wurde, lassen sich die Unterschiede direkt auf den Liganden zurückführen. Die Größe der erhaltenen RDCs ermöglicht in der Zukunft die Untersuchung von Proteindynamik.

Die bereits publizierten Tags CLaNP-5 und DOTA-M8 lieferten größere RDCs als Cys-Ph-TAHA (von bis zu $25 \mathrm{~Hz}$ bei $600 \mathrm{MHz}$ für CLaNP-5, beziehungsweise bis zu $20 \mathrm{~Hz}$ bei 800 MHz für DOTA-M8). Die großen RDCs, die mit CLaNP-5 bereits bei relativ niedrigem Magnetfeld erhalten wurden, beruhen jedoch auf der Verknüpfung des Tags über zwei Disulfidbrücken. Die Verwendung setzt demnach ein hohes Maß an Strukturkenntnis voraus, da zwei Cysteine in geeignetem Abstand voneinander eingeführt werden müssen. Die allgemeine Anwendbarkeit von CLaNP-5 wird somit erheblich eingeschränkt. Ziel dieser Arbeit war es jedoch, ein möglichst breit einsetzbares Werkzeug für die NMR-Spektroskopie zu entwickeln. Der direkte Vergleich muss deshalb eher zu DOTA-M8 gezogen werden. 
Mit DOTA-M8 wurde ein zweiter, temperaturabhängiger Datensatz mit einem Beitrag von $15-20 \%$ bei $298 \mathrm{~K}$ bis hin zu $50 \%$ bei $323 \mathrm{~K}$ beobachtet. Dies stellt einen nicht zu vernachlässigenden Einfluss auf die Spektren dar. Zwar waren in den HSQCSpektren von T12C Tb und T12C Tm ebenfalls Signale von zwei zusätzlichen Datensätzen auszumachen, jedoch verblieb deren Beitrag über den gesamten Temperaturbereich von 278-315 K konstant bei ungefähr 2\%. Da in den Spektren von S57C keine zusätzlichen Signale beobachtet wurden, lassen sich diastereomere Komplexe als Ursache für die weiteren Signalsätze in T12C auschließen. Cys-Ph-TAHA kann somit über einen weiten Temperaturbereich eingesetzt werden. Diese Eigenschaft wurde bislang für keinen Tag berichtet und könnte für die Untersuchung thermophiler Proteine von großem Nutzen sein. Im Gegensatz zu Cys-Ph-TAHA bietet DOTA-M8 die Möglichkeit, Messungen unterhalb von pH 4 durchführen zu können, da der käfigartige Ligand komplexierte Lanthanoidionen nicht wieder freisetzt.

Ein bedeutsamer Unterschied liegt in der synthetischen Zugänglichkeit der beiden Tags. Während Cys-Ph-TAHA in leicht großen Maßstäben hergestellt werden kann, ist bereits die Synthese des vierfach methylsubstituierten DOTA-Rings äußerst schwierig. Die größeren RDCs und die Möglichkeit der Messung unter pH 4 mit DOTA-M8 werden kompensiert, da mit Cys-Ph-TAHA über den gesamten untersuchten Temperaturbereich keine bedeutsamen Beiträge zusätzlicher Signalsätze, induziert durch diastereomere Lanthanoidkomplexe, auftreten.

Die reinen PCS- beziehungsweise RDC-abgeleiteten Tensoren weisen häufig deutliche Unterschiede in Größe und Orientierung auf. Innerhalb dieser Arbeit wurden verschiedene Möglichkeiten getestet, diese Tensoren miteinander zu verbinden. Durch die gleichzeitige Minimierung von RDCs und PCSs gegen einen gemeinsamen Tensor, wurde eine sehr gute Beschreibung für beide Datensätze erhalten. Diese neue Methode zur Tensorbestimmung erscheint somit sehr viel versprechend. In der Zukunft soll dieser Ansatz sowohl mit Datensätzen anderer Proteine, als auch durch Verwendung anderer Lanthanoide überprüft werden.

Es konnte gezeigt werden, dass RDCs weniger fehleranfällig als PCSs sind. Bislang fließen beide Parameter in gleichem Maße in die Tensorberechnung ein. Eine 
Gewichtung der Daten, die beispielsweise die unterschiedliche Empfindlichkeit auf Bewegungen des Tags berücksichtigt, sollte zu einem noch zuverlässigeren Tensor führen.

Neben weiteren Untersuchungen zu den Eigenschaften der Tensoren, ist ein Nahziel des Projekts die Synthese von weiteren Tags auf Grundlage des TAHA-Liganden, dessen Potential mit dieser Arbeit demonstriert wurde. Die nächste Generation des TAHA-Tags soll über einen axialsymmetrischen Linker verfügen, um die vorhandenen rotatorischen Freiheitsgrade zu reduzieren. Es ist zu erwarten, dass eine derartige Rigidifizierung des Tags in größeren RDCs resultiert.

Cys-Ph-TAHA soll zukünftig in verschiedenen Projekten verwendet werden. Der Tag wurde an mehrere Kooperationspartner verschickt und soll helfen, die jeweiligen Fragestellungen zu beantworten. Darüber hinaus besteht im Arbeitskreis ein großes Interesse an der Untersuchung von Oligonukleotiden. Es ist geplant, Thiolhaltige Nukleobasen in DNA-Sequenzen einzubauen, diese mit Cys-Ph-TAHA zu modifizieren und so die Anwendbarkeit paramagnetischer Tags auf Oligonukleotide zu erweitern. 


\section{Kapitel 6}

\section{Experimenteller Teil}

\subsection{Material und Methoden}

\section{Chemikalien:}

Alle Lösungsmittel wurden bei den Firmen Fluka oder Merck in p.a. Qualität gekauft. Für Reaktionen unter Inertgas wurden absolute und über Molekularsieb gelagerte Lösungsmittel verwendet. Die Chemikalien stammen von den Firmen Merck, Fluka, Sigma-Aldrich, Acros, Lancaster und Alfa Aesar. Als Inertgas wurde Argon verwendet, welches über einen Trockenturm (Phosphorpentoxid/Bimsstein) getrocknet wurde.

\section{Säulenchromatographie:}

Die Säulen wurden mit Kieselgel der Firma Merck gefüllt. Bei der Aufreinigung per Flash-Säulenchromatographie wurde eine Korngröße von 15-40 $\mu \mathrm{m}$ verwendet und bei einem Druck von 0.5-1.5 bar eluiert. Im Falle von Schwerkraftsäulen wurde Kieselgel der Korngröße 63-100 $\mu \mathrm{m}$ benutzt. Die Säulen wurden nass mit einem 50-100fachen Überschuss an Kieselgel befüllt und das Substanzgemisch als konzentrierte Lösung im Eluenten aufgetragen.

\section{Dünnschichtchromatographie:}

Reaktionskontrolle erfolgte mittels Dünnschichtchromatographie. Es wurden vorbeschichtete DC-Platten der Firma Machery-Nagel verwendet (0.25 mm Polygram 
SIL G/UV $\mathrm{U}_{24}$ ). Die Spots wurden durch Eintauchen in ethanolische Phosphormolybdänsäurelösung (10 wt\%) und anschließendes Erhitzen auf $200{ }^{\circ} \mathrm{C}$ visualisiert.

\section{Dialyse}

Es wurden Slide-A-Lyzer ${ }^{\circledR}$ Dialyse-Kassetten $(0.1-0.5 \mathrm{~mL}$ bzw. $0.5-3.0 \mathrm{~mL})$ von Thermo scientific mit 3.500 MWCO (molecular weight cutoff) verwendet.

\section{Probenkonzentration}

Zur Reduktion des Probenvolumens wurden Vivaspin 2 Konzentratoren mit PESMembran (2 mL, $5000 \mathrm{MWCO})$ von sartorius stedim biotech eingesetzt. Die Tischzentrifuge (Centrifuge 5804) stammt von der Firma Eppendorf.

\subsection{Charakterisierung der Substanzen}

\section{NMR-Spektren:}

Die NMR-Spektren zur Charakterisierung der synthetischen Verbindungen wurden an einem Bruker Avance Ultrashield-Spektrometer (400 MHz) bei einer Temperatur von $298 \mathrm{~K}$ aufgenommen. Die chemischen Verschiebungen $\delta$ wurden in ppm (parts per million) angegeben. Als interner Standard wurde auf die verbliebenen ${ }^{1} \mathrm{H}$-Resonanzsignale der deuterierten Lösungsmittel referenziert: $\mathrm{CDCl}_{3}: 7.26 \mathrm{ppm}$ $\left({ }^{1} \mathrm{H}-\mathrm{NMR}\right)$ und $77.0 \mathrm{ppm}\left({ }^{13} \mathrm{C}-\mathrm{NMR}\right), \mathrm{MeOH}_{-} \mathrm{d}_{4}: 3.31 \mathrm{ppm}\left({ }^{1} \mathrm{H}-\mathrm{NMR}\right)$ und $49.0 \mathrm{ppm}$ $\left({ }^{13} \mathrm{C}-\mathrm{NMR}\right)$, DMSO-d $\mathrm{d}_{6}: 2.49 \mathrm{ppm}\left({ }^{1} \mathrm{H}-\mathrm{NMR}\right)$ und $39.7 \mathrm{ppm}\left({ }^{13} \mathrm{C}-\mathrm{NMR}\right)$, Aceton- $\mathrm{d}_{6}$ : $2.05 \mathrm{ppm}\left({ }^{1} \mathrm{H}-\mathrm{NMR}\right)$ und $29.8 \mathrm{ppm}\left(\mathrm{CH}_{3},{ }^{13} \mathrm{C}-\mathrm{NMR}\right)$. Die Signale wurden wie folgt angegeben: chemische Verschiebung $\delta$ in ppm, Multiplizität $(\mathrm{s}=$ Singulett, $\mathrm{d}=\mathrm{Du}-$ blett, $\mathrm{t}=$ Triplett, $\mathrm{q}=$ Quartett, $\mathrm{m}=$ Multiplett, $\mathrm{br}=$ breites Signal), skalare Kopplungskonstante $J$ in Hertz, Integral und Kern. Die Signale wurden durch ${ }^{1} \mathrm{H},{ }^{13} \mathrm{C}-$ HSQC-Spektren, ${ }^{1} \mathrm{H},{ }^{13} \mathrm{C}-\mathrm{HMBC}-$ Spektren, sowie gegebenenfalls COSY-Spektren zugeordnet. 


\section{HPLC-Trennungen:}

Analytische und präparative HPLC-Trennungen wurden an Anlagen der Firma Jasco mit DAD-Detektor durchgeführt. Es wurden reversed phase-Säulen der Firma Knauer (Eurospher C18) eingesetzt. Bei analytischen Läufen wurden Säulen der Größe $150 \times 4.6 \mathrm{~mm}$, bei präparativen Trennungen Säulen von $250 \times 8 \mathrm{~mm}$ verwendet. Die analytischen Proben wurden bei einer Flussgeschwindigkeit von $1 \mathrm{~mL} / \mathrm{min}$, die präparativen mit $3 \mathrm{~mL} /$ min eluiert. Die reversed phase-Säulen wurden im Gradientenmodus betrieben $(100 \% \mathrm{~A} \rightarrow 100 \% \mathrm{~B})$. Als Laufmittel A wurde Wasser verwendet, B war Acetonitril. Beide Laufmittel enthielten jeweils 0.1\% Trifluoressigsäure. Der jeweilige Gradient ist in der Versuchsbeschreibung angegeben.

\section{Massenspektren:}

Die ESI-MS-Spektren wurden an einem Micromass ZQ 4000-Massenspektrometer mit Quadrupol-Detektor der Firma Waters aufgenommen. Die Angaben erfolgen in $m / z$. Die zu messenden Proben wurden in Methanol, Acetonitril oder Wasser gelöst. Zur Aufnahme von Massenspektren von Proteinen wurden diese gegen Ammoniumacetatpuffer $(20 \mathrm{mM})$ dialysiert.

\subsection{Protein-NMR}

\section{Ubiquitinproben}

Die Ubiquitinproben wurden hausintern vom Biolabor unter der Leitung von Dr. Stefan Becker angefertigt. Das Protein wurde rekombinant in E. coli exprimiert und gemäß etablierter Methoden aufgereinigt (Lazar et al. (1997); You et al. (1999)). Es wurden sowohl einfach $\left({ }^{15} \mathrm{~N}\right)$, als auch doppelt markierte Proben $\left({ }^{13} \mathrm{C},{ }^{15} \mathrm{~N}\right)$ zweier Ubiquitinmutanten (T12C und S57C) hergestellt. Um Dimerisierung durch Disulfidbrücken zu unterbinden, wurden die Proben TCEP-stabilisiert lyophylisiert. 


\section{Spektrenaufnahme:}

Die ${ }^{1} \mathrm{H},{ }^{15} \mathrm{~N}-\mathrm{HSQC}$ Spektren und HNCA-Spektren wurden an einem Avance III Spektrometer $(800 \mathrm{MHz}, 18.8 \mathrm{~T})$, ausgestattet mit Cryoplattform und CP-TCI Probenkopf mit z-Gradient, der Firma Bruker Biospin bei $298 \mathrm{~K}$ aufgenommen. IPAPHSQC- und 3D-HSQC-NOESY-Spektren wurden an einem Bruker Avance Spektrometer $(900 \mathrm{MHz}, 21.1 \mathrm{~T})$, ausgestattet mit CP-TCI Cryoprobenkopf mit z-Gradient, bei $298 \mathrm{~K}$ gemessen.

\section{Prozessierung:}

Die Spektren wurden mit dem Programmpaket NMRPipe prozessiert (Delaglio et al. (1995)). Die IPAP-Spektren wurden mit einem zero filling von 16k versehen, was bei einer spektralen Weite von 28 ppm in der indirekten Dimension zu einer digitalen Auflösung von $0.16 \mathrm{~Hz}$ führte.

\section{Auswertung der Ubiquitinspektren:}

Die zweidimensionalen Spektren wurden mit dem Programm Sparky (Goddard \& Kneller (2008)) ausgewertet. Für die dreidimensionalen Spektren wurde CARA (Keller (2004)) verwendet. Die sequentiellen Zuordnungen erfolgten hauptsächlich anhand der HNCA-Spektren. Der Magnetisierungstransfer im HNCA und der sequential walk durch das Spektrum im strip scope ist schematisch in Abbildung 6.1 gezeigt. Für einige Proben wurden zur Verifikation 3D-HSQC-NOESY Spektren hinzugezogen. 

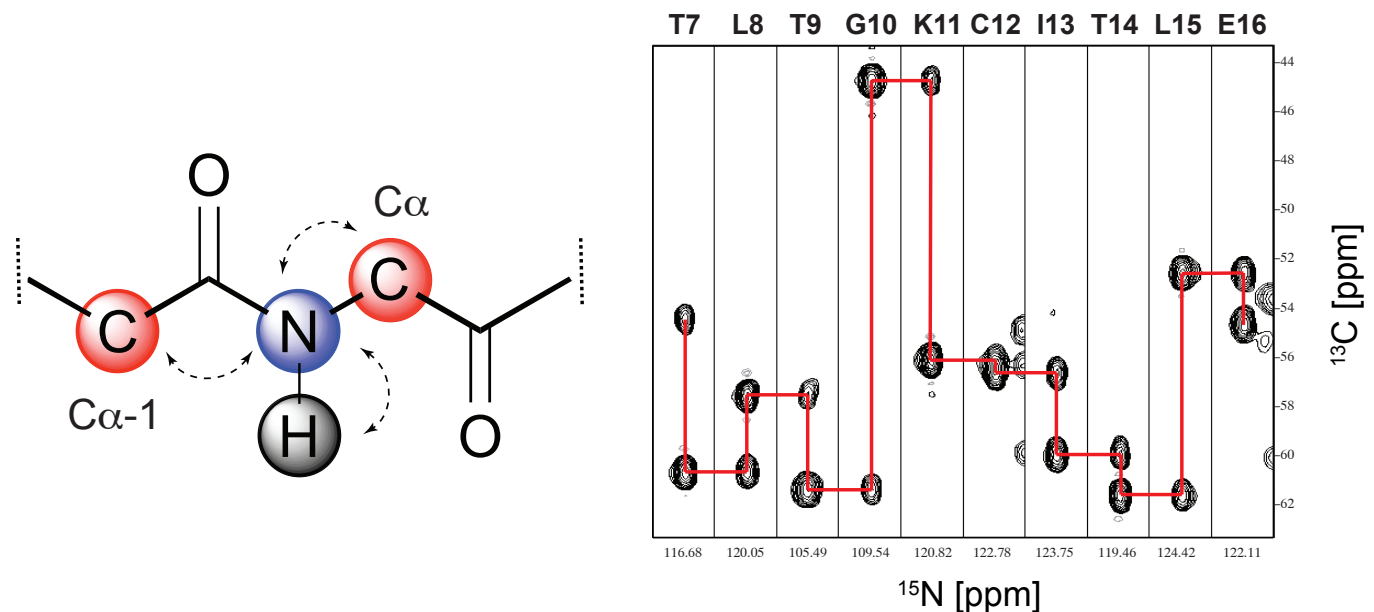

Abb. 6.1: Links: Schematische Darstellung der Magnetisierungsübertragungen und der erhaltenen Korrelationen im HNCA. Rechts: Das HNCA in der strip scopeDarstellung (CARA). Für einen gegebenen Aminosäurerest sind Korrelationen zwischen dessen Amidsignal und dem C $\alpha$ (stärkerer Peak) zu sehen. Darüber hinaus erscheint das $\mathrm{C} \alpha$ der vorausgehenden Aminosäure $(\mathrm{C} \alpha-1)$ als schwächerer Peak. Für den beispielhaften Ausschnitt von T7-E16 der T12C Mutante ist der sequential walk rot eingezeichnet. 


\section{IPAP-HSQC}

Um Signalüberlappungen zu minimieren, wurden die gekoppelten HSQC-Spektren unter Verwendung der IPAP-HSQC-Pulssequenz aufgenommen (in-phase-antiphaseHSQC, Ottiger et al. (1998)). Über den Phasenzyklus selektiert, werden zwei gekoppelte HSQC-Spektren aufgezeichnet, wobei die heteronukleare Kopplung im einen Fall als Inphase-, im anderen Fall als Antiphasedublett detektiert wird. Durch anschließende Addition, beziehungsweise Subtraktion werden HSQC-Spektren erhalten, deren Peaks jeweils um die halbe Kopplungskonstante verschoben sind und somit die gewünschte Information ohne eine Verdopplung des Signalsatzes enthalten. Dies ist in Abbildung 6.2 schematisch dargestellt.

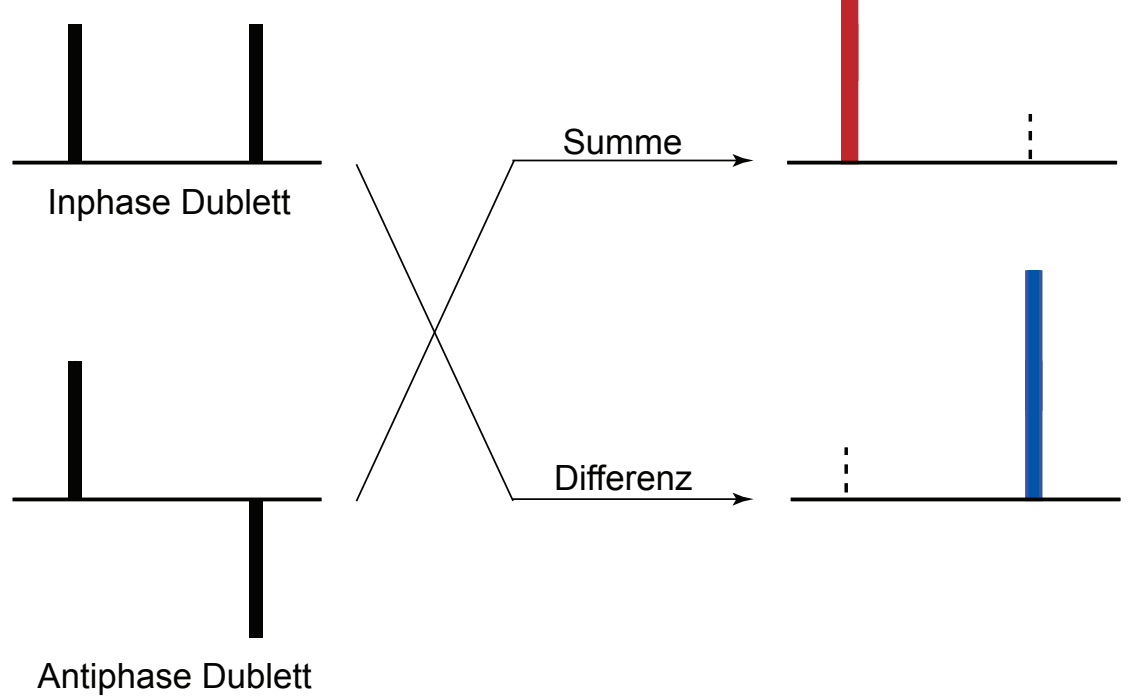

Abb. 6.2: Schematische Darstellung der Prozessierung des IPAP-HSQC. Die Signale in den erhaltenen Spektren, dargestellt in rot (Summe) und blau (Differenz), sind jeweils um die halbe Kopplungskonstante tief- bzw. hochfeldverschoben. 


\subsection{Synthesevorschriften}

\subsubsection{Synthese von 2-(4-Bromphenyl)acetaldehyd}<smiles>OCCc1ccc(Br)cc1</smiles>

28

201.06

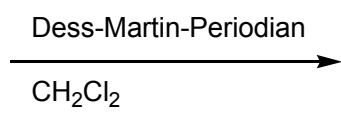

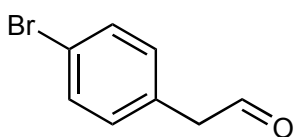

29

199.04

2-(4-Bromphenyl)ethanol 28 (2.40 g, $11.94 \mathrm{mmol})$ wurde in abs. Dichlormethan (80 mL) aufgenommen, mit Dess-Martin-Periodinan (5.46 g, 12.87 mmol, 1.08 Äq.) versetzt und die Suspension unter Argon und Lichtausschluss bei Raumtemperatur für 2.5 h gerührt. Anschließend wurden 100 mL einer gesättigten Natriumhydrogencarbonatlösung und 25 \%ige Natriumthiosulfatlösung $(30 \mathrm{~mL})$ zugefügt und weitere 15 min gerührt. Die Phasen wurden getrennt, die wässrige Phase mit Dichlormethan extrahiert $(3 \times 50 \mathrm{~mL})$, die vereinte organische Phase über Natriumsulfat getrocknet und im Vakuum eingeengt. Das Produkt 29 wurde quantitativ als gelbe Flüssigkeit erhalten, die bei Raumtemperatur erstarrte.

${ }^{1} \mathbf{H}-\mathrm{NMR}\left(\mathrm{CDCl}_{3}, 400 \mathrm{MHz}\right): \delta=9.60(\mathrm{~s}, 1 \mathrm{H}, \mathrm{CHO}), 7.45(\mathrm{~d}, J=8.3 \mathrm{~Hz}, 2 \mathrm{H}$, H-3', H-5'), 7.05 (d, J = 8.3 Hz, 2H, H-2', H-6'), 3.62 (s, 2H, H-2) ppm.

${ }^{13}$ C-NMR $\left(\mathrm{CDCl}_{3}, 100.6 \mathrm{MHz}\right): \delta=198.5$ (1C, CHO), 132.0 (2C, C-3', C-5'), 131.3 (2C, C-2', C-6'), 130.9 (1C, C-1'), 121.4 (1C, C-4'), 49.5 (1C, C-2) ppm. 


\subsubsection{Synthese von 4-Brom- $\alpha, \alpha, \alpha$-tris(hydroxymethyl)toluol}<smiles>O=CCc1ccc(Br)cc1</smiles>

29

199.04

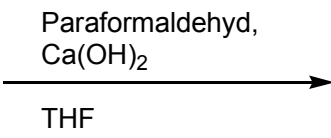
Unter Argon wurde eine Suspension von Verbindung 29 (2.37 g, $11.91 \mathrm{mmol}) \mathrm{mit}$
Paraformaldehyd (3.07 g, $102.23 \mathrm{mmol}, 8.6$ Äq.) und Calciumhydroxid (10.12 g, $136.59 \mathrm{mmol}, 11.5$ Äq. $)$ in abs. Tetrahydrofuran $(150 \mathrm{~mL})$ bei $65^{\circ} \mathrm{C}$ für 90 h gerührt. Die abgekühlte Suspension wurde durch Celite filtriert und mit Tetrahydrofuran gewaschen, bis das Filtrat farblos wurde. Das Lösungsmittel wurde im Vakuum entfernt, der Rückstand säulenchromatographisch an Kieselgel gereinigt (Eluent: Hexan/Aceton 1:1) und $\mathbf{3 0}$ (1.44 g, $5.51 \mathrm{mmol}, 46 \%)$ in Form eines farblosen Öls erhalten.

${ }^{1} \mathbf{H}-\mathrm{NMR}\left(\mathrm{CDCl}_{3}, 400 \mathrm{MHz}\right): \delta=7.49(\mathrm{~d}, J=8.6 \mathrm{~Hz}, 2 \mathrm{H}, \mathrm{H}-3, \mathrm{H}-5), 7.26(\mathrm{~d}$, $J=8.6 \mathrm{~Hz}, 2 \mathrm{H}, \mathrm{H}-2, \mathrm{H}-6), 4.09\left(\mathrm{~s}, 6 \mathrm{H}, 3 \times \mathrm{CH}_{2}\right) 2.37(\mathrm{br} \mathrm{s}, 3 \mathrm{H}, 3 \times \mathrm{OH}) \mathrm{ppm}$.

${ }^{13}$ C-NMR $\left(\mathrm{CDCl}_{3}, 100.6 \mathrm{MHz}\right): \delta=138.54$ (1C, C-1), 132.0 (2C, C-3, C-5), 128.6 (2C, C-2, C-6), 120.9 (1C, C-4), $66.4\left(3 \mathrm{C}, 3 \times \mathrm{CH}_{2}\right), 48.6$ (1C, C- $\left.\alpha\right)$ ppm.

ESI-MS $m / z$ (Methanol, positive mode): ber. für $\mathrm{C}_{10} \mathrm{H}_{13}{ }^{79} \mathrm{BrNaO}_{3}[\mathrm{M}+\mathrm{Na}]^{+}: 282.99$, gef.: 282.88 . 


\subsubsection{Synthese von 4-Brom- $\alpha, \alpha, \alpha$-tris(tosyloxymethyl)toluol}<smiles>OCC(CO)(CO)c1ccc(Br)cc1</smiles>

30

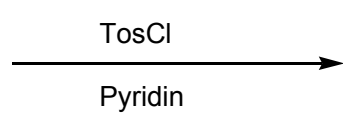

261.11

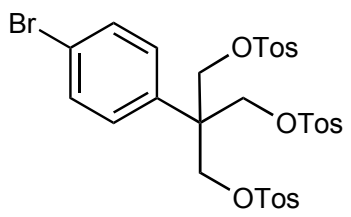

31

30 (4.70 g, $18.00 \mathrm{mmol}$ ) wurde in $120 \mathrm{~mL}$ abs. Pyridin aufgenommen, mit Tosylchlorid (20.60 g, 108.05 mmol, 10.0 Äq.) versetzt und bei Raumtemperatur für 60 h gerührt. Das Pyridiniumchlorid wurde abfiltriert und das Pyridin im Vakuum entfernt. Zum Rückstand wurden Wasser $(250 \mathrm{~mL})$ und Dichlormethan $(250 \mathrm{~mL})$ gegeben und anschließend die Phasen getrennt. Die wässrige Phase wurde mit Dichlormethan extrahiert $(3 \times 200 \mathrm{~mL})$, die vereinigte organische Phase über Natriumsulfat getrocknet und im Vakuum eingeengt. Die säulenchromatographische Reinigung an Kieselgel (Eluent: Dichlormethan) lieferte die Zielverbindung 31 (11.85 g, $16.37 \mathrm{mmol}, 91 \%$ ) als weißen Schaum.

${ }^{1} \mathbf{H}-\mathbf{N M R}\left(\mathrm{CDCl}_{3}, 400 \mathrm{MHz}\right): \delta=7.59(\mathrm{~d}, J=8.2 \mathrm{~Hz}, 6 \mathrm{H}, 6 \times$ Tos-Ar $), 7.30(\mathrm{~d}$, $J=8.2 \mathrm{~Hz}, 6 \mathrm{H}, 6 \times$ Tos-Ar), $7.22(\mathrm{~d}, J=8.6 \mathrm{~Hz}, 2 \mathrm{H}, \mathrm{H}-3, \mathrm{H}-5), 6.76(\mathrm{~d}, J=8.6 \mathrm{~Hz}$, $2 \mathrm{H}, \mathrm{H}-2, \mathrm{H}-6), 4.14\left(\mathrm{~s}, 6 \mathrm{H}, 3 \times \mathrm{CH}_{2}\right), 2.46\left(\mathrm{~s}, 9 \mathrm{H}, 3 \times \mathrm{CH}_{3}\right) \mathrm{ppm}$.

${ }^{13}$ C-NMR $\left(\mathrm{CDCl}_{3}, 100.6 \mathrm{MHz}\right): \delta=145.4(3 \mathrm{C}, 3 \times$ Tos-Ar), 133.8 (1C, C-1), 131.8 (2C, C-3, C-5), 131.5 (3C, $3 \times$ Tos-Ar), 130.0 (6C, $6 \times$ Tos-Ar), 127.8 (8C, C-2, C-6, $6 \times$ Tos-Ar), $122.2(1 \mathrm{C}, \mathrm{C}-4), 68.4\left(3 \mathrm{C}, 3 \times \mathrm{CH}_{2}\right), 45.9(1 \mathrm{C}, \mathrm{C}-\alpha), 21.6(3 \mathrm{C}$, $\left.3 \times \mathrm{CH}_{3}\right)$ ppm.

ESI-MS $m / z$ (Methanol, positive mode): ber. für $\mathrm{C}_{31} \mathrm{H}_{31}{ }^{79} \mathrm{BrNaO}_{9} \mathrm{~S}_{3}[\mathrm{M}+\mathrm{Na}]^{+}$: 745.02, gef.: 744.94 . 


\subsubsection{Synthese von 4-Brom- $\alpha, \alpha, \alpha$-tris(azidomethyl)toluol}<smiles>OCC(CO)(CO[Na])c1ccc(Br)cc1</smiles>

31

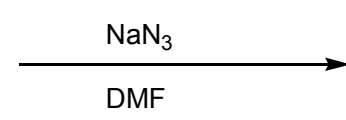

723.67

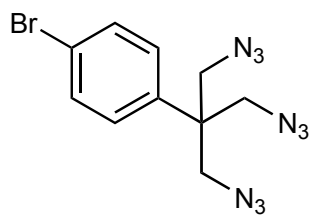

32

336.15

31 (11.50 g, $15.89 \mathrm{mmol}$ ) wurde in $500 \mathrm{~mL}$ abs. Dimethylformamid aufgenommen, mit Natriumazid (6.19 g, $95.23 \mathrm{mmol}, 6.0$ Äq.) versetzt und bei $100{ }^{\circ} \mathrm{C}$ für $18 \mathrm{~h}$ gerührt. Anschließend wurden zur abgekühlten Suspension 1400 mL Wasser gegeben und diese mit Dichlormethan extrahiert $(3 \times 500 \mathrm{~mL})$. Die vereinte organische Phase wurde mit Wasser gewaschen $(500 \mathrm{~mL})$ und über Natriumsulfat getrocknet. Das Lösungsmittel wurde im Vakuum bis auf einen Rest von $30 \mathrm{~mL}$ entfernt, abs. Tetrahydrofuran zugegeben $(50 \mathrm{~mL})$ und das Volumen im Vakuum auf einen Rest von $30 \mathrm{~mL}$ reduziert. Dies wurde wiederholt $(2 \times)$ und die so erhaltene Lösung in die nächste Stufe eingesetzt. 


\subsubsection{Synthese von 4-Brom- $\alpha, \alpha, \alpha$-tris(aminomethyl)toluol}<smiles>NCC(CN)(CN)c1ccc(Br)cc1</smiles>

32

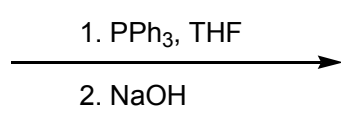

336.15

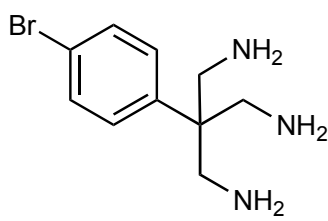

33

258.16

Die Lösung von 32 wurde unter Argon mit Tetrahydrofuran auf $400 \mathrm{~mL}$ aufgefüllt, mit Triphenylphosphin (37.53 g, 143.10 mmol, 9.0 Äq.) versetzt und bei $65{ }^{\circ} \mathrm{C}$ für 2 h gerührt. Nach Ende der Gasentwicklung wurde Natronlauge (2M, $195 \mathrm{~mL})$ zugefügt und die Lösung für weitere $2 \mathrm{~h}$ bei $65{ }^{\circ} \mathrm{C}$ gerührt. Im Anschluss wurde die Reaktionslösung mit Salzsäure auf pH 1 angesäuert und die wässrige Phase mit Chloroform gewaschen $(3 \times 300 \mathrm{~mL})$. Danach wurde die wässrige Phase mit festem Natriumhydroxid auf pH 12 eingestellt und mit Chloroform extrahiert $(5 \times 300 \mathrm{~mL})$. Die organische Phase wurde vereint, über Natriumsulfat getrocknet und im Vakuum eingeengt. Das Produkt 33 (3.80 g, 14.72 mmol, 93\% über 2 Stufen) wurde als gelbliches Öl erhalten.

${ }^{1}$ H-NMR $\left(\mathrm{MeOH}_{-} \mathrm{d}_{4}, 400 \mathrm{MHz}\right): \delta=7.55$ (d, $\left.J=8.6 \mathrm{~Hz}, 2 \mathrm{H}, \mathrm{H}-3, \mathrm{H}-5\right), 7.30$ (d, $J=8.6 \mathrm{~Hz}, 2 \mathrm{H}, \mathrm{H}-2, \mathrm{H}-6), 2.90\left(\mathrm{~s}, 6 \mathrm{H}, 3 \times \mathrm{CH}_{2}\right) \mathrm{ppm}$.

${ }^{13}$ C-NMR (MeOH-d 4 , 100.6 MHz): $\delta=141.4$ (1C, C-1), 131.4 (2C, C-3, C-5), 128.6 (2C, C-2, C-6), $120.6(1 \mathrm{C}, \mathrm{C}-4), 48.3(1 \mathrm{C}, \mathrm{C}-\alpha), 45.0\left(3 \mathrm{C}, 3 \times \mathrm{CH}_{2}\right) \mathrm{ppm}$.

ESI-MS $m / z$ (Methanol, positive mode): ber. für $\mathrm{C}_{10} \mathrm{H}_{17}{ }^{79} \mathrm{BrN}_{3}[\mathrm{M}+\mathrm{H}]^{+}:$258.06, gef.: 257.98 . 


\subsubsection{Synthese von 4-Brom- $\alpha, \alpha, \alpha$-tris $[[N, N$-di $($ tert-butoxy- carbonylmethyl)amino]methyl]toluol}<smiles>NCC(CN)(CN)c1ccc(Br)cc1</smiles>

33

258.16

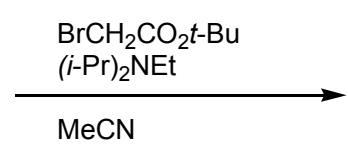

34

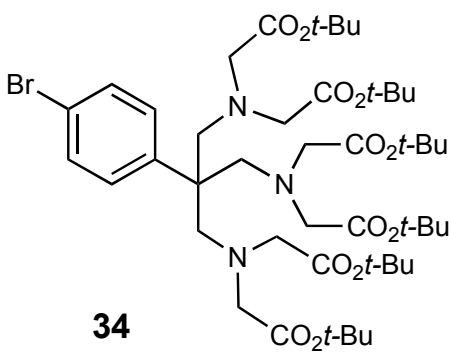

943.01

Die Verbindung 33 (3.30 g, $12.78 \mathrm{mmol})$ wurde unter Argon in abs. Acetonitril (330 mL) gelöst, mit Diisopropylethylamin (26.75 mL, $156.27 \mathrm{mmol}, 12.2$ Äq.) und Bromessigsäure-tert-butylester (17.0 mL, $115.05 \mathrm{mmol}, 9.0$ Äq.) versetzt und unter Rückfluss für 42 h gerührt. Das Lösungsmittel wurde im Vakuum entfernt und der Rückstand in Dichlormethan gelöst. Die Lösung wurde mit 5 \%iger Zitronensäurelösung $(2 \times 200 \mathrm{~mL})$ und Wasser $(200 \mathrm{~mL})$ gewaschen, über Natriumsulfat getrocknet und im Vakuum eingeengt. Die Reinigung erfolgte säulenchromatographisch an Kieselgel (Eluent: Dichlormethan/Methanol 100:1). Das Produkt 34 wurde als gelber Schaum (11.69 g, $12.40 \mathrm{mmol}, 97 \%$ ) erhalten.

${ }^{1}$ H-NMR $\left(\mathrm{CDCl}_{3}, 400 \mathrm{MHz}\right): \delta=7.31(\mathrm{~d}, J=8.7 \mathrm{~Hz}, 2 \mathrm{H}, \mathrm{H}-3, \mathrm{H}-5), 7.25$ (d, $J=8.7 \mathrm{~Hz}, 2 \mathrm{H}, \mathrm{H}-2, \mathrm{H}-6), 3.17\left(\mathrm{~s}, 18 \mathrm{H}, 9 \times \mathrm{CH}_{2}\right), 1.39\left(\mathrm{~s}, 54 \mathrm{H}, 6 \times \mathrm{C}_{\left.\left(\mathrm{CH}_{3}\right)_{3}\right) \mathrm{ppm} .}\right.$

${ }^{13} \mathbf{C}-\mathbf{N M R}\left(\mathrm{CDCl}_{3}, 100.6 \mathrm{MHz}\right): \delta=170.9\left(6 \mathrm{C}, 6 \times \mathrm{CO}_{2} t-\mathrm{Bu}\right), 143.7(1 \mathrm{C}, \mathrm{C}-1)$, $131.3(2 \mathrm{C}, \mathrm{C}-3, \mathrm{C}-5), 128.6(2 \mathrm{C}, \mathrm{C}-2, \mathrm{C}-6), 119.8(1 \mathrm{C}, \mathrm{C}-4), 80.4\left(6 \mathrm{C}, 6 \times C\left(\mathrm{CH}_{3}\right)_{3}\right)$, $59.3\left(3 \mathrm{C}, 3 \times \mathrm{C}-\alpha \mathrm{CH}_{2}\right), 56.5\left(6 \mathrm{C}, 6 \times \mathrm{NCH}_{2} \mathrm{CO}_{2} t-\mathrm{Bu}\right), 48.5(1 \mathrm{C}, \mathrm{C}-\alpha), 28.0(18 \mathrm{C}$, $\left.18 \times \mathrm{C}\left(\mathrm{CH}_{3}\right)_{3}\right) \mathrm{ppm}$.

ESI-MS $m / z$ (Methanol, positive mode): ber. für $\mathrm{C}_{46} \mathrm{H}_{77}{ }^{79} \mathrm{BrN}_{3} \mathrm{O}_{12}[\mathrm{M}+\mathrm{H}]^{+}:$: 944.47 , gef.: 944.44 . 


\subsubsection{Synthese von $\alpha, \alpha, \alpha$-Tris $[[N, N$-di(tert-butoxycarbonyl- methyl)amino]methyl]-p-toluolsäure}<smiles>COC(=O)CN(CC(C)(C)C)CC(CN(CCN(CC(=O)OC(C)(C)C)CC(C)(C)C)CC(=O)OC(C)(C)C)(CN(CC(=O)OC)CC(C)(C)C)c1ccc(Br)cc1</smiles>

943.01

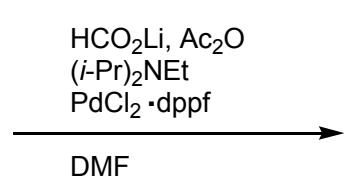<smiles>COC(=O)CN(CC(C)(C)C)CC(CN(CCN(CC(=O)OC(C)(C)C)CC(C)(C)C)CC(=O)OC(C)(C)C)(CN(CC(=O)OC)CC(C)(C)C)c1ccc(C(=O)O)cc1</smiles>

908.13

Die Verbindung 34 (360 mg, $382 \mu \mathrm{mol})$ wurde in einem Druckkolben mit Schraubdeckel in $6.5 \mathrm{~mL}$ abs. Dimethylformamid gelöst, mit getrocknetem Lithiumformiat (179 mg, $3.44 \mathrm{mmol}, 9.0$ Äq.) und $\mathrm{PdCl}_{2} \cdot \operatorname{dppf}(28 \mathrm{mg}, 36.2 \mu \mathrm{mol}, 10 \mathrm{~mol} \%$ ) versetzt. Es wurde für 20 min Argon durch die Suspension geleitet und anschließend Essigsäureanhydrid (216 $\mu \mathrm{L}, 2.29$ mmol, 6.0 Äq.) und Diisopropylethylamin (392 $\mu \mathrm{L}$, 2.29 mmol, 6.0 Äq.) hinzugefügt. Der Kolben wurde unter Argonatmosphäre fest verschlossen und bei $120{ }^{\circ} \mathrm{C}$ für $44 \mathrm{~h}$ gerührt. Das abgekühlte Reaktionsgemisch wurde auf Essigsäureethylester $(80 \mathrm{~mL})$ gegossen und die organische Phase mit Wasser $(2 \times 50 \mathrm{~mL})$ gewaschen. Die organische Phase wurde über Natriumsulfat getrocknet und im Vakuum eingeengt. Das erhaltene Rohprodukt wurde säulenchromatographisch an Kieselgel gereinigt (Eluent: Dichlormethan/Methanol 20:1) und das Produkt (282 mg, $311 \mu \mathrm{mol}, 81 \%)$ als braunes Öl erhalten.

${ }^{1} \mathbf{H}-\mathbf{N M R}\left(\mathrm{CDCl}_{3}, 400 \mathrm{MHz}\right): \delta=7.99(\mathrm{~d}, J=8.2 \mathrm{~Hz}, 2 \mathrm{H}, \mathrm{H}-3, \mathrm{H}-5), 7.55$ (d, $J=8.2 \mathrm{~Hz}, 2 \mathrm{H}, \mathrm{H}-2, \mathrm{H}-6), 3.31\left(\mathrm{~s}, 6 \mathrm{H}, 3 \times \mathrm{C}-\alpha \mathrm{CH}_{2}\right), 3.22\left(\mathrm{~s}, 12 \mathrm{H}, 6 \times \mathrm{CH}_{2} \mathrm{CO}_{2} t-\mathrm{Bu}\right)$, $1.42\left(\mathrm{~s}, 54 \mathrm{H}, 6 \times \mathrm{C}\left(\mathrm{CH}_{3}\right)_{3}\right) \mathrm{ppm}$. 
${ }^{13} \mathbf{C}-\mathrm{NMR}\left(\mathrm{CDCl}_{3}, 100.6 \mathrm{MHz}\right): \delta=170.0\left(7 \mathrm{C}, 6 \times C \mathrm{O}_{2} t-\mathrm{Bu}, \mathrm{C}-4 C \mathrm{O}_{2} \mathrm{H}\right), 151.3$ (1C, C-1), 130.2 (2C, C-3, C-5), 126.9 (2C, C-2, C-6), 127.3 (1C, C-4), 80.6 (6C, $6 \times$ $\left.C\left(\mathrm{CH}_{3}\right)_{3}\right), 59.5\left(3 \mathrm{C}, 3 \times \mathrm{C}-\alpha \mathrm{CH}_{2}\right), 56.6\left(6 \mathrm{C}, 6 \times \mathrm{NCH}_{2} \mathrm{CO}_{2} t-\mathrm{Bu}\right), 49.4(1 \mathrm{C}, \mathrm{C}-\alpha)$, $28.1\left(18 \mathrm{C}, 18 \times \mathrm{C}\left(\mathrm{CH}_{3}\right)_{3}\right)$ ppm.

ESI-MS m/z (Methanol, positive mode): ber. für $\mathrm{C}_{47} \mathrm{H}_{78} \mathrm{~N}_{3} \mathrm{O}_{14}[\mathrm{M}+\mathrm{H}]^{+}:$908.55, gef.: 908.57 . 


\subsubsection{Synthese von $N$ - $[4-[\alpha, \alpha, \alpha$-Tris $[[N, N$-di $($ tert-butoxycar- bonylmethyl)amino]methyl]methyl]benzoyl]-S-mesyl- $(R)$-cystein}<smiles>COC(=O)CN(CC(=O)OC(C)(C)C)CC(CN(CCOC(C)(C)C)CC(=O)OCC(C)(C)C)(CN(CC(=O)OC)CC(C)(C)C)c1ccc(C(=O)O)cc1</smiles>

35

908.13

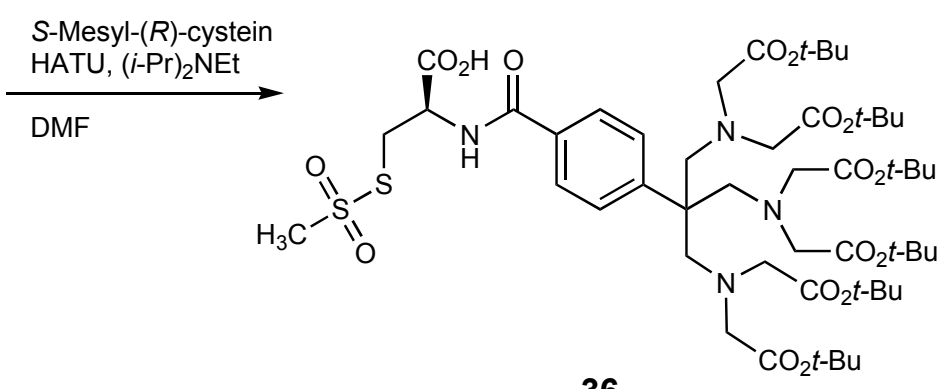

36

Die Verbindung 35 (178 mg, $196 \mu \mathrm{mol})$ wurde in $10 \mathrm{~mL}$ abs. Dimethylformamid aufgenommen, mit HATU (75 mg, $196 \mu \mathrm{mol}, 1.0$ Äq.) und Diisopropylethylamin (33.5 $\mu \mathrm{L}, 196 \mu \mathrm{mol}, 1.0$ Äq.) versetzt und bei $25^{\circ} \mathrm{C}$ für $2.5 \mathrm{~h}$ gerührt. Anschließend wurde $S$-Mesyl-( $R$ )-cystein (39 mg, $196 \mu \mathrm{mol}, 1.0$ Äq.) und erneut Diisopropylethylamin $\left(33.5 \mu \mathrm{L}, 196 \mu \mathrm{mol}, 1.0\right.$ Äq.) zugegeben und für weitere $20 \mathrm{~h}$ bei $25^{\circ} \mathrm{C}$ gerührt. Danach wurde das Lösungsmittel im Vakuum entfernt und zum Rückstand jeweils $30 \mathrm{~mL}$ Wasser und Chloroform gegeben. Die Phasen wurden getrennt, die wässrige Phase mit Chloroform $(2 \times 20 \mathrm{~mL})$ extrahiert, die vereinte organische Phase über Natriumsulfat getrocknet und im Vakuum eingeengt. Das erhaltene Rohprodukt wurde säulenchromatographisch an Kieselgel gereinigt (Eluent: Dichlormethan/Methanol 25:1 auf 8:1) und das Produkt 36 (147 mg, $135 \mu \mathrm{mol}, 69 \%$ ) als braunes Öl erhalten. Darüber hinaus wurde 35 (31 mg, $34 \mu \mathrm{mol}, 17 \%)$ reisoliert.

${ }^{1} \mathbf{H}-\mathrm{NMR}\left(\mathrm{CDCl}_{3}, 400 \mathrm{MHz}\right): \delta=8.02(\mathrm{~s}, 1 \mathrm{H}, \mathrm{NH}), 7.82(\mathrm{~s}, 2 \mathrm{H}, \mathrm{H}-3, \mathrm{H}-5), 7.52(\mathrm{~s}$, 2H, H-2, H-6), 4.71-4.89 (m, 1H, $\left.\mathrm{SCH}_{2} \mathrm{CH}\right), 3.60-4.00$ (m, 2H, $\left.\mathrm{SCH}_{2}\right), 3.36(\mathrm{~s}, 3 \mathrm{H}$, $\left.\mathrm{SCH}_{3}\right), 3.28\left(\mathrm{~s}, 6 \mathrm{H}, 3 \times \mathrm{C}-\alpha \mathrm{CH}_{2}\right), 3.22\left(\mathrm{~s}, 12 \mathrm{H}, 6 \times \mathrm{NCH}_{2} \mathrm{CO}_{2} t-\mathrm{Bu}\right), 1.43(\mathrm{~s}, 54 \mathrm{H}$, $\left.6 \times \mathrm{C}\left(\mathrm{CH}_{3}\right)_{3}\right) \mathrm{ppm}$. 
${ }^{13} \mathrm{C}-\mathrm{NMR}\left(\mathrm{CDCl}_{3}, 100.6 \mathrm{MHz}\right): \delta=171.0\left(6 \mathrm{C}, 6 \times \mathrm{CO}_{2} t-\mathrm{Bu}\right), 167.6\left(1 \mathrm{C}, \mathrm{CO}_{2} \mathrm{H}\right)$, 162.9 (1C, NHCO), 149.3 (1C, C-1), 130.6 (1C, C-4), 127.6 (2C, C-3, C-5), 126.9 $(2 \mathrm{C}, \mathrm{C}-2, \mathrm{C}-6), 80.7\left(6 \mathrm{C}, 6 \times C\left(\mathrm{CH}_{3}\right)_{3}\right), 59.6\left(3 \mathrm{C}, 3 \times \mathrm{C}-\alpha C \mathrm{H}_{2}\right), 56.6(6 \mathrm{C}, 6 \times$ $\left.\mathrm{NCH}_{2} \mathrm{CO}_{2} t-\mathrm{Bu}\right), 54.6\left(1 \mathrm{C}, \mathrm{SCH}_{2} C \mathrm{H}\right), 50.3(1 \mathrm{C}, \mathrm{C}-\alpha), 49.2\left(1 \mathrm{C}, \mathrm{SCH}_{3}\right), 34.7(1 \mathrm{C}$, $\left.\mathrm{SCH}_{2} \mathrm{CH}\right), 28.2\left(18 \mathrm{C}, 18 \times \mathrm{C}\left(\mathrm{CH}_{3}\right)_{3}\right) \mathrm{ppm}$.

ESI-MS $m / z$ (Methanol, positive mode): ber. für $\mathrm{C}_{51} \mathrm{H}_{85} \mathrm{~N}_{4} \mathrm{O}_{17} \mathrm{~S}_{2}[\mathrm{M}+\mathrm{H}]^{+}:$1089.54, gef.: 1089.49 . 


\subsubsection{Synthese von $N$-[4- $[\alpha, \alpha, \alpha$-Tris $[[N, N$-di(carboxymethyl)- amino]methyl]methyl]benzoyl]-S-mesyl- $(R)$-cystein, Cys-Ph-TAHA}

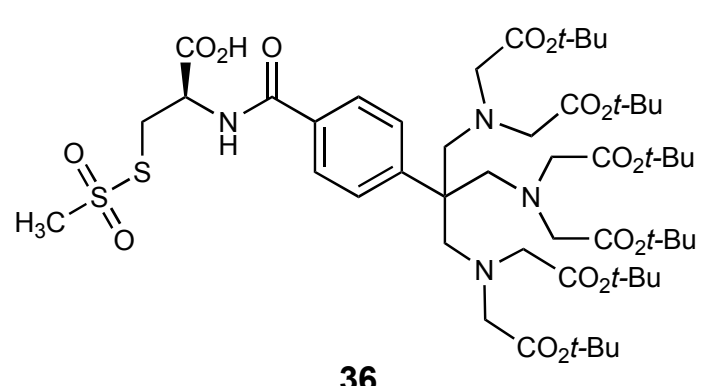

1089.36<smiles>CC(C)CC(=O)O</smiles>

37

752.72

Die Verbindung 36 (209 mg, $192 \mu \mathrm{mol})$ wurde in $20 \mathrm{~mL}$ konzentrierter Trifluoressigsäure aufgenommen und bei $25{ }^{\circ} \mathrm{C}$ für $18 \mathrm{~h}$ gerührt. Anschließend wurde im Vakuum eingeengt. Der Rückstand wurde in $16 \mathrm{~mL}$ Wasser aufgenommen und die unlöslichen Bestandteile durch Zentrifugieren abgetrennt. Die Lösung wurde per präparativer HPLC gereinigt. Der verwendete Gradient ist unten angegeben. Die Produktfraktionen (Retentionszeit von $16.5 \mathrm{~min}$ ) wurden vereint und lyophylisiert. Cys-Ph-TAHA 37 wurde als weißes Pulver (89 mg, $118 \mu \mathrm{mol}, 62 \%$ ) erhalten.

${ }^{1} \mathbf{H}-\mathrm{NMR}\left(\mathrm{D}_{2} \mathrm{O}, 400 \mathrm{MHz}\right): \delta=7.81(\mathrm{~d}, J=7.9 \mathrm{~Hz}, 2 \mathrm{H}, \mathrm{H}-3, \mathrm{H}-5), 7.66(\mathrm{~d}$, $J=7.9 \mathrm{~Hz}, 2 \mathrm{H}, \mathrm{H}-2, \mathrm{H}-6), 4.86-4.95\left(\mathrm{~m}, 1 \mathrm{H}, \mathrm{SCH}_{2} \mathrm{CH}\right), 3.83(\mathrm{~d}, J=13.8 \mathrm{~Hz}, 1 \mathrm{H}$, $\left.\mathrm{SCH}_{a} \mathrm{H}_{b}\right), 3.69-3.61\left(\mathrm{~m}, 7 \mathrm{H}, \mathrm{SCH}_{a} H_{b}, 3 \times \mathrm{C}-\alpha \mathrm{CH}_{2}\right), 3.56\left(\mathrm{~s}, 12 \mathrm{H}, 6 \times \mathrm{NCH}_{2} \mathrm{CO}_{2} \mathrm{H}\right)$, $3.42\left(\mathrm{~s}, 3 \mathrm{H}, \mathrm{CH}_{3}\right) \mathrm{ppm}$.

${ }^{13}$ C-NMR $\left(\mathrm{D}_{2} \mathrm{O}, 100.6 \mathrm{MHz}\right): \delta=172.5\left(7 \mathrm{C}, 7 \times \mathrm{CO}_{2} \mathrm{H}\right), 169.3(1 \mathrm{C}, \mathrm{C}-4 C \mathrm{O}), 143.3$ (1C, C-1), 131.9 (1C, C-4), 127.9 (2C, C-3, C-5), 126.8 (2C, C-2, C-6), 60.7 (3C, $\left.3 \times \mathrm{C}-\alpha C \mathrm{H}_{2}\right), 55.7\left(6 \mathrm{C}, 6 \times \mathrm{NCH}_{2} \mathrm{CO}_{2} \mathrm{H}\right), 52.6\left(1 \mathrm{C}, \mathrm{SCH}_{2} C \mathrm{H}\right), 49.4\left(1 \mathrm{C}, \mathrm{CH}_{3}\right)$, $46.1(1 \mathrm{C}, \mathrm{C}-\alpha), 36.2\left(1 \mathrm{C}, \mathrm{S} C \mathrm{H}_{2} \mathrm{CH}\right) \mathrm{ppm}$. 
ESI-MS m/z (Wasser, positive mode): ber. für $\mathrm{C}_{27} \mathrm{H}_{37} \mathrm{~N}_{4} \mathrm{O}_{17} \mathrm{~S}_{2}[\mathrm{M}+\mathrm{H}]^{+}:$: 753.16 , gef.: 753.08 .

ESI-HRMS $m / z$ (Wasser, positive mode): ber. für $\mathrm{C}_{27} \mathrm{H}_{37} \mathrm{~N}_{4} \mathrm{O}_{17} \mathrm{~S}_{2}[\mathrm{M}+\mathrm{H}]^{+}:$: 753.1590 , gef.: 753.1588 .

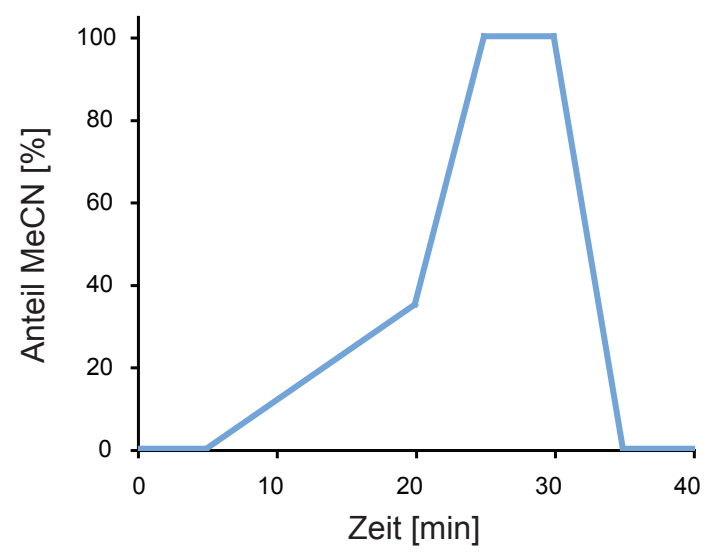

Abb. 6.3: Gradient der Trennung. Wasser und Acetonitril enthielten jeweils $0.1 \%$ TFA.

Aufgetragen ist der Anteil an Acetonitril gegen die Zeit. 


\subsection{Präparation von getaggtem, beladenem Ubi- quitin}

Für die Herstellung einer NMR Probe wurden $3 \mathrm{mg}$ des Proteinlyophylisats einer einfach bzw. doppelt markierten Ubiquitinmutante (T12C oder S57C) in $200 \mu \mathrm{L}$ Puffer (MOPS 50 mM, NaCl 50 mM, pH 8.0) aufgenommen, in eine Dialyse-Kassette (Slide-A-Lyzer ${ }^{\circledR}, 0.1-0.5 \mathrm{~mL}, 3500 \mathrm{MWCO}$ ) gefüllt und für 4 h gegen $1000 \mathrm{~mL}$ des oben genannten Puffers dialysiert, um die Reduktionsmittel in Form von TCEP zu entfernen.

Parallel wurden die wässrigen Stammlösungen des Tags Cys-Ph-TAHA (37, 5.0 Äq., $\mathrm{c}=10 \mathrm{mg} / \mathrm{mL})$ und Lanthanoidtrichlorid (10.0 Äq, c $=10 \mathrm{mg} / \mathrm{mL})$ zusammen pipettiert und für $2.5 \mathrm{~h}$ bei Raumtemperatur inkubiert. Anschließend wurden die beiden Lösungen vereint. Nach 75 min wurde das ausgefallene Lanthanoidtrihydroxid durch Zentrifugieren entfernt und der Überstand in einen Vivaspin 2 Konzentrator mit PES-Membran (2 mL, 5000 MWCO) gegeben. Dieser wurde mit dem späteren Messpuffer (MOPS 50 mM, NaCl 50 mM, pH 6.8) aufgefüllt und bei 5000 rcf (relative centrifugal force) auf ein Volumen von $220 \mu \mathrm{L}$ reduziert. Dieser Vorgang wurde wiederholt $(9 \times)$ und so die überschüssigen, komplexierten Lanthanoide entfernt. Die Proteinlösung wurde in ein Shigemi NMR-Röhrchen überführt und mit $30 \mu \mathrm{L}$ $\mathrm{D}_{2} \mathrm{O}$ versetzt.

Zur Kontrolle der Taggingreaktion wurde ein kleiner Anteil gegen Ammoniumactetatpuffer $(20 \mathrm{mM})$ dialysiert und per Massenspektrometrie qualitativ ermittelt, ob noch ungetaggtes Protein vorliegt. 


\section{Literaturverzeichnis}

Aime, S., M. Botta, M. Fasano, M. P. Marques, C. F. Geraldes, D. Pubanz, \& A. E. Merbach (1997). Conformational and coordination equilibria on DOTA complexes of lanthanide metal ions in aqueous solution studied by ${ }^{1} \mathrm{H}-\mathrm{NMR}$ spectroscopy. Inorg. Chem. 36, 2059-2068.

Allegrozzi, M., I. Bertini, M. B. L. Janik, G. Lee, Y. M. Liu, \& C. Luchinat (2000). Lanthanide-induced pseudocontact shifts for solution structure refinements of macromolecules in shells up to $40 \AA$ from the metal ion. J. Am. Chem. Soc. 122, $4154-4161$.

Alpoim, M. C., A. M. Urbano, C. F. G. C. Geraldes, \& J. A. Peters (1992). Determination of the number of inner-sphere water molecules in lanthanide(III) polyaminocarboxylate complexes. J. Chem. Soc. Dalton Trans. 1992, 463-467.

Alsaadi, B. M., F. J. C. Rossotti, \& R. J. P. Williams (1980). Electron relaxation rates of lanthanide aquo-cations. J. Chem. Soc. Dalton Trans. 11, 2147-2150.

Arnesano, F., L. Banci, I. Bertini, K. Van der Wetering, M. Czisch, \& R. Kaptein (2000). The auto-orientation in high magnetic fields of oxidized cytochrome $b_{562}$ as source of constraints for solution structure determination. J. Biomol. NMR 17, $295-304$.

Arslantas, E., P. M. Smith-Jones, G. Ritter, \& R. R. Schmidt (2004). TAME-Hex A - a novel bifunctional chelating agent for radioimmunoimaging. Eur. J. Org. Chem. 2004, 3979-3984. 
Bailey, A. J., W. P. Griffith, S. I. Mostafa, \& P. A. Sherwood (1993). Studies on transition-metal oxo and nitrido complexes. Perruthenate and ruthenate anions as catalytic organic oxidants. Inorg. Chem. 32, 268-271.

Ban, N., P. Nissen, J. Hansen, P. B. Moore, \& T. A. Steitz (2000). The complete atomic structure of the large ribosomal subunit at $2.4 \AA$ resolution. Science $\mathbf{2 8 9}$, 905-20.

Barry, C. D., A. C. North, J. A. Glasel, R. J. Williams, \& A. V. Xavier (1971). Quantitative determination of mononucleotide conformations in solution using lanthanide ion shift and broadenine NMR probes. Nature 232, 236-245.

Bax, A., G. Kontaxis, \& N. Tjandra (2001). Dipolar couplings in macromolecular structure determination. Methods Enzymol. 339, 127-174.

Bax, A. \& N. Tjandra (1997). High-resolution heteronuclear NMR of human ubiquitin in an aqueous liquid crystalline medium. J. Biomol. NMR 10, 289-292.

Bayrhuber, M., T. Meins, M. Habeck, S. Becker, K. Giller, S. Villinger, C. Vonrhein, C. Griesinger, M. Zweckstetter, \& K. Zeth (2008). Structure of the human voltagedependent anion channel. Proc. Natl. Acad. Sci. USA 105, 15370-15375.

Berger, P., A. Bessmernykh, J. C. Caille, \& S. Mignonac (2006). Palladium-catalyzed hydroxycarbonylation of aryl and vinyl bromides by mixed acetic formic anhydride. Synthesis 18, 3106-3110.

Bertini, I., C. Del Bianco, I. Gelis, N. Katsaros, C. Luchinat, G. Parigi, M. Peana, A. Provenzani, \& M. A. Zoroddu (2004). Experimentally exploring the conformational space sampled by domain reorientation in calmodulin. Proc. Natl. Acad. Sci. USA 101, 6841-6846.

Bertini, I., I. Gelis, N. Katsaros, C. Luchinat, \& A. Provenzani (2003). Tuning the affinity for lanthanides of calcium binding proteins. Biochemistry 42, 8011-8021.

Bertini, I., M. B. L. Janik, Y. M. Lee, C. Luchinat, \& A. Rosato (2001). Magnetic susceptibility tensor anisotropies for a lanthanide ion series in a fixed protein matrix. J. Am. Chem. Soc. 123, 4181-4188. 
Bertini, I. \& C. Luchinat (1999). New applications of paramagnetic NMR in chemical biology. Curr. Opin. Chem. Biol. 3, 145-151.

Bertini, I., C. Luchinat, \& G. Parigi (2002). Magnetic susceptibility in paramagnetic NMR. Prog. Nucl. Magn. Res. Spec. 40, 249-273.

Bleaney, B. (1972). Nuclear magnetic resonance shifts in solution due to lanthanide ions. J. Magn. Res. 8, 91-100.

Bunnage, M. E., A. J. Burke, S. G. Davies, N. L. Millican, R. L. Nicholson, P. M. Roberts, \& A. D. Smith (2003). Asymmetric synthesis of anti-(2S,3S)- and syn(2R,3S)-diaminobutanoic acid. Org. Biomol. Chem. 1, 3708-3715.

Cacchi, S., G. Fabrizi, \& A. Goggiamani (2003). Palladium-catalyzed hydroxycarbonylation of aryl and vinyl halides or triflates by acetic anhydride and formate anions. Org. Lett. 5, 4269-4272.

Chiu, F. C. K., R. T. C. Brownlee, K. Mitchell, \& D. R. Phillips (1995). 9Aminoacridine-EDTA conjugates as hydroxy radical footprinting reagents with no intrinsic cutting specificity. Bioorg. \& Med. Chem. Lett. 5, 1689-1694.

Cornilescu, G., J. L. Marquardt, M. Ottiger, \& A. Bax (1998). Validation of protein structure from anisotropic carbonyl chemical shifts in a dilute liquid crystalline phase. J. Am. Chem. Soc. 120, 6836-6837.

Dalcanale, E. \& F. Montanari (1986). Selective oxidation of aldehydes to carboxylic acids with sodium chlorite-hydrogen peroxide. J. Org. Chem. 51, 567-569.

Datwyler, S. A. \& C. F. Meares (2000). Protein-protein interactions mapped by artificial proteases: where sigma factors bind to RNA polymerase. Trends Biochem. Sci. 25, 408-414.

Delaglio, F., S. Grzesiek, G. W. Vuister, G. Zhu, J. Pfeifer, \& A. Bax (1995). NMRPipe: a multidimensional spectral processing system based on UNIX pipes. $J$. Biomol. NMR 6, 277-293. 
Dess, D. B. \& J. C. Martin (1983). Readily accessible 12-I-5 oxidant for the conversion of primary and secondary alcohols to aldehydes and ketones. J. Org. Chem. 48, $4155-4156$.

Djanashvili, K., C. Platas-Iglesias, \& J. A. Peters (2008). The structure of the lanthanide aquo ions in solution as studied by ${ }^{17} \mathrm{O}$ NMR spectroscopy and DFT calculations. Dalton Trans. 5, 602-607.

Dvoretsky, A., V. Gaponenko, \& P. R. Rosevear (2002). Derivation of structural restraints using a thiol-reactive chelator. FEBS Lett. 528, 189-192.

Ebright, Y. W., Y. Chen, P. S. Pendergrast, \& R. H. Ebright (1992). Incorporation of an EDTA-metal complex at a rationally selected site within a protein: application to EDTA-iron DNA affinity cleaving with catabolite gene activator protein (CAP) and Cro. Biochemistry 31, 10664-10670.

Ellman, G. L. (1959). Tissue sulfhydryl groups. Arch. Biochem. Biophys. 82, 70-77.

Ermacora, M. R., J. M. Delfino, B. Cuenoud, A. Schepartz, \& R. O. Fox (1992). Conformation-dependent cleavage of staphylococcal nuclease with a disulfidelinked iron chelate. Proc. Natl. Acad. Sci. U.S.A 89, 6383-6387.

Evans, C. H. (1990). Biochemistry of the lanthanides. Plenum Press, New York, 1. Auflage.

Franklin, S. J. \& K. N. Raymond (1994). Solution structure and dynamics of lanthanide complexes of the macrocyclic polyamino carboxylate DTPA-dien. NMR study and crystal structures of the lanthanum(III) and europium(III) complexes. Inorg. Chem. 33, 5794-5804.

Franz, K. J., M. Nitz, \& B. Imperiali (2003). Lanthanide-binding tags as versatile protein coexpression probes. ChemBioChem. 4, 265-271.

Gaponenko, V., A. S. Altieri, J. Li, \& R. A. Byrd (2002). Breaking symmetry in the structure determination of (large) symmetric protein dimers. J. Biomol. NMR 24, $143-148$. 
Gaponenko, V., A. Dvoretsky, C. Walsby, B. M. Hoffman, \& P. R. Rosevear (2000). Calculation of z-coordinates and orientational restraints using a metal binding tag. Biochemistry 39, 15217-15224.

Goddard, T. D. \& D. G. Kneller (2008). Sparky 3. University of California, San Francisco.

Griesinger, C., O. W. Sorensen, \& R. Ernst (1987). Novel three-dimensional NMR techniques for studies of peptides and biological macromolecules. J. Am. Chem. Soc. 109, 7227-7228.

Gueron, M. (1975). Nuclear relaxation in macromolecules by paramagnetic ions: a novel mechanism. J. Magn. Res. 19, 58-66.

Haberz, P., J. Farjon, \& C. Griesinger (2005). A DMSO-compatible orienting medium: towards the investigation of the stereochemistry of natural products. Angew. Chem. Int. Ed. 44, 427-429.

Haberz, P., F. Rodriguez-Castañeda, J. Junker, S. Becker, A. Leonov, \& C. Griesinger (2006). Two new chiral EDTA-based metal chelates for weak alignment of proteins in solution. Org. Lett. 8, 1275-1278.

Hanessian, S. \& P. Lavallee (1975). The preparation and synthetic utility of tertbutyldiphenylsilyl ethers. Can. J. Chem. 53, 2975-2977.

Hansen, M. R., L. Mueller, \& A. Pardi (1998). Tunable alignment of macromolecules by filamentous phage yields dipolar coupling interactions. Nat. Struct. Biol. 5, 1065-1074.

Hart, T. W. (1985). Some observations concerning the $S$-nitroso and $S$-phenylsulphonyl derivatives of L-cysteine and glutathione. Tetrahedron Lett. 26, 20132016.

Häussinger, D., J. R. Huang, \& S. Grzesiek (2009). DOTA-M8: An extremely rigid, high-affinity lanthanide chelating tag for PCS NMR spectroscopy. J. Am. Chem. Soc. 131, 14761-14767. 
Hellmann, K. W., S. Friedrich, L. H. Gade, W. S. Li, \& M. McPartlin (1995). Tripodal Triamidostannates as building blocks in the generation of Sn-M-bonded heterobimetallics $(\mathrm{M}=\mathrm{Fe}, \mathrm{Ru})$. Chem. Ber. 128, 29-34.

Hershko, A. \& A. Ciechanover (1992). The ubiquitin system for protein degradation. Annu. Rev. Biochem. 61, 761-807.

Hershko, A. \& A. Ciechanover (1998). The ubiquitin system. Annu. Rev. Biochem. 67, 425-479.

Hershko, A., A. Ciechanover, H. Heller, A. L. Haas, \& I. A. Rose (1980). Proposed role of ATP in protein breakdown: conjugation of protein with multiple chains of the polypeptide of ATP-dependent proteolysis. Proc. Natl. Acad. Sci. USA 77, $1783-1786$.

Hudson, A. \& J. W. E. Lewis (1970). Electron spin relaxation of ${ }^{8} S$ ions in solution. Trans. Faraday Soc. 66, 1297-1301.

Hus, J. C., D. Marion, \& M. Blackledge (2000). De novo determination of protein structure by NMR using orientational and long-range order restraints. J. Mol. Biol. 298, 927-936.

Ikegami, T., L. Verdier, P. Sakhaii, S. Grimme, B. Pescatore, K. Saxena, K. M. Fiebig, \& C. Griesinger (2004). Novel techniques for weak alignment of proteins in solution using chemical tags coordinating lanthanide ions. J. Biomol. NMR 29, 339-349.

Ikura, M., L. E. Kay, \& A. Bax (1990). A novel approach for sequential assignment of ${ }^{1} \mathrm{H},{ }^{13} \mathrm{C}$, and ${ }^{15} \mathrm{~N}$ spectra of proteins: heteronuclear triple-resonance threedimensional NMR spectroscopy. Application to calmodulin. Biochemistry 29, 4659-4667.

Keizers, P. H. J., J. F. Desreux, M. Overhand, \& M. Ubbink (2007). Increased paramagnetic effect of a lanthanide protein probe by two-point attachment. $J$. Am. Chem. Soc. 129, 9292-9293. 
Keizers, P. H. J., A. Saragliadis, Y. Hiruma, M. Overhand, \& M. Ubbink (2008). Design, synthesis, and evaluation of a lanthanide chelating protein probe: CLaNP5 yields predictable paramagnetic effects independent of environment. J. Am. Chem. Soc. 130, 14802-14812.

Keller, R. L. J. (2004). Optimizing the process of nuclear magnetic resonance spectrum analysis and computer aided resonance assignment. Dissertation, Swiss Federal Institute of Technology, Zürich.

Kobzar, K., H. Kessler, \& B. Luy (2005). Stretched gelatin gels as chiral alignment media for the discrimination of enantiomers by NMR spectroscopy. Angew. Chem. Int. Ed. 44, 3145-3147.

Kramer, F., M. V. Deshmukh, H. Kessler, \& S. J. Glaser (2004). Residual dipolar coupling constants: an elementary derivation of key equations. Concepts Magn. Res. Part A 21 A, 10-21.

Kubota, Y., T. Hanaoka, K. Takeuchi, \& Y. Sugi (1994). An efficient synthesis of aryl esters by palladium-catalyzed carbonylation of 4-bromobiphenyl. Synlett $\mathbf{7}$, $515-517$.

Kummerlöwe, G., J. Auernheimer, A. Lendlein, \& B. Luy (2007). Stretched poly(acrylonitrile) as a scalable alignment medium for DMSO. J. Am. Chem. Soc. 129, 6080-6081.

Kurland, R. J. \& B. R. McGarvey (1970). Isotropic NMR shifts in transition metal complexes: the calculation of the fermi contact and pseudocontact terms. J. Magn. Res. 2, 286-301.

Lakomek, N. A., T. Carlomagno, S. Becker, C. Griesinger, \& J. Meiler (2006). A thorough dynamic interpretation of residual dipolar couplings in ubiquitin. $J$. Biomol. NMR 34, 101-115.

Lakomek, N. A., O. F. Lange, K. F. A. Walter, C. Farès, D. Egger, P. Lunkenheimer, J. Meiler, H. Grubmüller, S. Becker, B. L. de Groot, \& C. Griesinger (2008). 
Residual dipolar couplings as a tool to study molecular recognition of ubiquitin. Biochem. Soc. Trans. 36, 1433-1437.

Lakomek, N. A., K. F. A. Walter, C. Farès, O. F. Lange, B. L. de Groot, H. Grubmüller, R. Brüschweiler, A. Munk, S. Becker, J. Meiler, \& C. Griesinger (2008). Self-consistent residual dipolar coupling based model-free analysis for the robust determination of nanosecond to microsecond protein dynamics. $J$. Biomol. NMR 41, 139-155.

Lange, O. F., N. A. Lakomek, C. Farès, G. F. Schröder, K. F. A. Walter, S. Becker, J. Meiler, H. Grubmüller, C. Griesinger, \& B. L. de Groot (2008). Recognition dynamics up to microseconds revealed from an RDC-derived ubiquitin ensemble in solution. Science 320, 1471-1475.

Lazar, G. A., J. R. Desjarlais, \& T. M. Handel (1997). De novo design of the hydrophobic core of ubiquitin. Protein Sci. 6, 1167-1178.

Lenz, R. \& S. V. Ley (1997). Tetra- $N$-propylammonium perruthenate (TPAP)catalysed oxidations of alcohols using molecular oxygen as a co-oxidant. J. Chem. Soc., Perkin Trans. 1 18, 3291-3292.

Leonov, A., B. Voigt, F. Rodriguez-Castañeda, P. Sakhaii, \& C. Griesinger (2005). Convenient synthesis of multifunctional EDTA-based chiral metal chelates substituted with an S-mesylcysteine. Chemistry 11, 3342-3348.

Ley, S. V., J. Norman, W. P. Griffith, \& S. P. Marsden (1994). Tetrapropylammonium perruthenate, $\mathrm{Pr}_{4} \mathrm{~N}^{+} \mathrm{RuO}_{4}^{-}$, TPAP: a catalytic oxidant for organic synthesis. Synthesis 7, 639-666.

Liang, B., J. H. Bushweller, \& L. K. Tamm (2006). Site-directed parallel spinlabeling and paramagnetic relaxation enhancement in structure determination of membrane proteins by solution NMR spectroscopy. J. Am. Chem. Soc. 128, 43894397. 
Lindfors, H. E., P. E. de Koning, J. W. Drijfhout, B. Venezia, \& M. Ubbink (2008). Mobility of TOAC spin-labelled peptides binding to the Src SH3 domain studied by paramagnetic NMR. J. Biomol. NMR 41, 157-167.

Losonczi, J. A., M. Andrec, M. W. F. Fischer, \& J. H. Prestegard (1999). Order matrix analysis of residual dipolar couplings using singular value decomposition. J. Magn. Reson. 138, 334-342.

Man, B., X. C. Su, H. Liang, S. Simonsen, T. Huber, B. A. Messerle, \& G. Otting (2010). 3-Mercapto-2,6-pyridinedicarboxylic acid: a small lanthanide-binding tag for protein studies by NMR spectroscopy. Chem. Eur. J. 16, 3827-3832.

Mancuso, A. J., S. L. Huang, \& D. Swern (1978). Oxidation of long-chain and related alcohols to carbonyls by dimethyl sulfoxide activated by oxalyl chloride. J. Org. Chem. 43, 2480-2482.

Martin, L. J., M. J. Hähnke, M. Nitz, J. Wöhnert, N. R. Silvaggi, K. N. Allen, H. Schwalbe, \& B. Imperiali (2007). Double-lanthanide-binding tags: design, photophysical properties, and NMR applications. J. Am. Chem. Soc. 129, 7106-7113.

Marx, A. \& C. Thiele (2009). Orientational properties of poly-gamma-benzyl-Lglutamate: influence of molecular weight and solvent on order parameters of the solute. Chemistry 15, 254-260.

McConnell, H. M. \& R. E. Robertson (1958). Isotropic nuclear resonance shifts. J. Chem. Phys. 29, 1361-1365.

McGarvey, B. R. (1979). Temperature dependence of the pseudocontact shift in lanthanide shift reagents. J. Magn. Res. 33, 445-455.

Meiler, J., W. Peti, \& C. Griesinger (2003). Dipolar couplings in multiple alignments suggest $\alpha$ helical motion in ubiquitin. J. Am. Chem. Soc. 125, 8072-8073.

Nitz, M., K. J. Franz, R. L. Maglathlin, \& B. Imperiali (2003). A powerful combinatorial screen to identify high-affinity terbium(III)-binding peptides. ChemBioChem. 4, 272-276. 
Nitz, M., M. Sherawat, K. J. Franz, E. Peisach, K. N. Allen, \& B. Imperiali (2004). Structural origin of the high affinity of a chemically evolved lanthanide-binding peptide. Angew. Chem. Int. Ed. 43, 3682-3685.

Oschkinat, H., C. Griesinger, P. J. Kraulis, O. W. Sorensen, R. R. Ernst, A. M. Gronenborn, \& G. M. Clore (1988). Three-dimensional NMR spectroscopy of a protein in solution. Nature 332, 374-376.

Ottiger, M., F. Delaglio, \& A. Bax (1998). Measurement of $J$ and dipolar couplings from simplified two-dimensional NMR spectra. J. Magn. Reson. 131, 373-378.

Ottiger, M., F. Delaglio, J. L. Marquardt, N. Tjandra, \& A. Bax (1998). Measurement of dipolar couplings for methylene and methyl sites in weakly oriented macromolecules and their use in structure determination. J. Magn. Reson. 134, 365-369.

Otting, G. (2008). Prospects for lanthanides in structural biology by NMR. $J$. Biomol. NMR 42, 1-9.

Otting, G. (2010). Protein NMR using paramagnetic ions. Annu. Rev. Biophys. 39, $387-405$.

Peti, W., J. Meiler, R. Brüschweiler, \& C. Griesinger (2002). Model-free analysis of protein backbone motion from residual dipolar couplings. J. Am. Chem. Soc. 124, $5822-5833$.

Pihko, A. J., K. C. Nicolaou, \& A. M. P. Koskinen (2001). An expedient synthesis of D-callipeltose. Tetrahedron Assym. 12, 937-942.

Pintacuda, G., M. John, X. C. Su, \& G. Otting (2007). NMR structure determination of protein-ligand complexes by lanthanide labeling. Acc. Chem. Res. 40, 206-212.

Pintacuda, G., M. A. Keniry, T. Huber, A. Y. Park, N. E. Dixon, \& G. Otting (2004). Fast structure-based assignment of ${ }^{15} \mathrm{~N}$ HSQC spectra of selectively ${ }^{15} \mathrm{~N}$-labeled paramagnetic proteins. J. Am. Chem. Soc. 126, 2963-2970. 
Pintacuda, G., A. Moshref, A. Leonchiks, A. Sharipo, \& G. Otting (2004). Sitespecific labelling with a metal chelator for protein-structure refinement. J. Biomol. NMR 29, 351-361.

Pintacuda, G., A. Y. Park, M. A. Keniry, N. E. Dixon, \& G. Otting (2006). Lanthanide labeling offers fast NMR approach to 3D structure determinations of protein-protein complexes. J. Am. Chem. Soc. 128, 3696-3702.

Polasek, M., J. Rudovsky, P. Hermann, I. Lukes, L. V. Elst, \& R. N. Muller (2004). Lanthanide(III) complexes of a pyridine $N$-oxide analogue of DOTA: exclusive $M$ isomer formation induced by a six-membered chelate ring. Chem. Commun. 22, 2602-2603.

Prestegard, J. H., H. M. Al-Hashimi, \& J. R. Tolman (2000). NMR structures of biomolecules using field oriented media and residual dipolar couplings. Q. Rev. Biophys. 33, 371-424.

Prudêncio, M., J. Rohovec, J. A. Peters, E. Tocheva, M. J. Boulanger, M. E. P. Murphy, H. J. Hupkes, W. Kosters, A. Impagliazzo, \& M. Ubbink (2004). A caged lanthanide complex as a paramagnetic shift agent for protein NMR. Chem. Eur. J. 10, 3252-3260.

Raach, A. \& O. Reiser (2000). Sodium chlorite-hydrogen peroxide - a mild and selective reagent for the oxidation of aldehydes to carboxylic acids. J. Prakt. Chem. 342, 605-608.

Ranganathan, R. S., R. K. Pillai, N. Raju, H. Fan, H. Nguyen, M. F. Tweedle, J. F. Desreux, \& V. Jacques (2002). Polymethylated DOTA ligands. 1. Synthesis of rigidified ligands and studies on the effects of alkyl substitution on acid-base properties and conformational mobility. Inorg. Chem. 41, 6846-6855.

Ranganathan, R. S., N. Raju, H. Fan, X. Zhang, M. F. Tweedle, J. F. Desreux, \& V. Jacques (2002). Polymethylated DOTA ligands. 2. Synthesis of rigidified lanthanide chelates and studies on the effect of alkyl substitution on conformational mobility and relaxivity. Inorg. Chem. 41, 6856-6866. 
Rodriguez-Castañeda, F., P. Haberz, A. Leonov, \& C. Griesinger (2006). Paramagnetic tagging of diamagnetic proteins for solution NMR. Magn. Reson. Chem.44, $10-16$.

Rückert, M. \& G. Otting (2000). Alignment of biological macromolecules in novel nonionic liquid crystalline media for NMR experiments. J. Am. Chem. Soc. 122, $7793-7797$.

Rye, C. S. \& S. G. Withers (2002). Synthesis and evaluation of potential inhibitors of chondroitin AC lyase from flavobacterium heparinum. J. Org. Chem. 67, 45054512.

Saito, S., H. Nakajima, M. Inaba, \& T. Moriwake (1989). One-pot transformation of azido-group to $N$-(t-butoxycarbonyl)amino group. Tetrahedron Lett. 30, 837-838.

Sanders, C. R., B. J. Hare, K. P. Howard, \& J. H. Prestegard (1994). Magneticallyoriented phospholipid micelles as a tool for the study of membrane-associated molecules. Prog. NMR Spect. 26, 421-444.

Sanders, 2nd, C. R. \& J. H. Prestegard (1990). Magnetically orientable phospholipid bilayers containing small amounts of a bile salt analogue, CHAPSO. Biophys. J. 58, 447-460.

Sanders, J. K. M. \& D. H. Williams (1970). A shift reagent for use in nuclear magnetic resonance spectroscopy. A first-order spectrum of $n$-hexanol. Chem. Commun 7, 422-423.

Saupe, A. (1968). Recent results in the field of liquid crystals. Angew. Chem. Int. Ed. 7, 97-112.

Schmitt, J., H. Hess, \& H. G. Stunnenberg (1993). Affinity purification of histidinetagged proteins. Mol. Biol. Rep. 18, 223-230.

Shimomura, O., F. H. Johnson, \& Y. Saiga (1962). Extraction, purification and properties of aequorin, a bioluminescent protein from the luminous hydromedusan, Aequorea. J. Cell. Comp. Physiol. 59, 223-239. 
Sieber, P. (1977). Der 2-Trimethylsilyläthyl-Rest als selektiv abspaltbare CarboxySchutzgruppe. Helv. Chim. Acta 60, 2711-2716.

Sigl, A. \& H. Sigl (2003). Metal ions in biological systems. 40, Marcel Dekker, Inc, Basel, 1. Auflage.

Staudinger, H. \& J. Meyer (1919). Über neue organische Phosphorverbindungen III. Phosphinmethylenderivate und Phosphinimine. J. Helv. Chim. Acta 2, 635-646.

Su, X. C., T. Huber, N. E. Dixon, \& G. Otting (2006). Site-specific labelling of proteins with a rigid lanthanide-binding tag. ChemBioChem. 7, 1599-1604.

$\mathrm{Su}$, X. C., H. Liang, K. V. Loscha, \& G. Otting (2009). [ $\left.\operatorname{Ln}(\mathrm{DPA})_{3}\right]^{3-}$ is a convenient paramagnetic shift reagent for protein NMR studies. J. Am. Chem. Soc. 131, 10352-10353.

Su, X. C., B. Man, S. Beeren, H. Liang, S. Simonsen, C. Schmitz, T. Huber, B. A. Messerle, \& G. Otting (2008). A dipicolinic acid tag for rigid lanthanide tagging of proteins and paramagnetic NMR spectroscopy. J. Am. Chem. Soc. 130, 1048610487.

Su, X. C., K. McAndrew, T. Huber, \& G. Otting (2008). Lanthanide-binding peptides for NMR measurements of residual dipolar couplings and paramagnetic effects from multiple angles. J. Am. Chem. Soc. 130, 1681-1687.

Su, X. C. \& G. Otting (2010). Paramagnetic labelling of proteins and oligonucleotides for NMR. J. Biomol. NMR. 46, 101-112.

Thiele, C. M. \& S. Berger (2003). Probing the diastereotopicity of methylene protons in strychnine using residual dipolar couplings. Org. Lett. 5, 705-708.

Tolman, J. R., J. M. Flanagan, M. A. Kennedy, \& J. H. Prestegard (1995). Nuclear magnetic dipole interactions in field-oriented proteins: information for structure determination in solution. Proc. Natl. Acad. Sci. USA 92, 9279-9283.

Tsien, R. Y. (1998). The green fluorescent protein. Annu. Rev. Biochem. 67, 509544. 
Tüchelmann, A., H. Schwalbe, \& C. Griesinger (1998). Third European Conference Oxford, Stable Isotope aided NMR of Biomolecules. Poster.

Tycko, R., F. J. Blanco, \& Y. Ishii (2000). Alignment of biopolymers in strained gels: A new way to create detectable dipole-dipole couplings in high-resolution biomolecular NMR. J. Am. Chem. Soc. 122, 9340-9341.

Viguier, R., G. Serratrice, A. Dupraz, \& C. Dupuy (2001). New polypodal polycarboxylic ligands - complexation of rare-earth ions in aqueous solution. Eur. J. Inorg. Chem. 2001, 1789-1795.

Vlasie, M. D., C. Comuzzi, A. M. C. H. van den Nieuwendijk, M. Prudêncio, M. Overhand, \& M. Ubbink (2007). Long-range-distance NMR effects in a protein labeled with a lanthanide-DOTA chelate. Chem. Eur. J. 13, 1715-1723.

Weidner, J. P. \& S. S. Block (1972). Mixed thiolsulfonates and sulfonamides from polyfunctional mercaptans using trifluoromethyl thiosulfonates. J. Med. Chem. 15, $564-567$.

Wöhnert, J., K. J. Franz, M. Nitz, B. Imperiali, \& H. Schwalbe (2003). Protein alignment by a coexpressed lanthanide-binding tag for the measurement of residual dipolar couplings. J. Am. Chem. Soc. 125, 13338-13339.

Wöltjen, E. (2009). Paramagnetisch markierte Oligonukleotide. Dissertation, GeorgAugust-Universität Göttingen.

Xu, X., P. H. J. Keizers, W. Reinle, F. Hannemann, R. Bernhardt, \& M. Ubbink (2009). Intermolecular dynamics studied by paramagnetic tagging. J. Biomol. NMR 43, 247-254.

Xu, X., W. Reinle, F. Hannemann, P. V. Konarev, D. I. Svergun, R. Bernhardt, \& M. Ubbink (2008). Dynamics in a pure encounter complex of two proteins studied by solution scattering and paramagnetic NMR spectroscopy. J. Am. Chem. Soc. 130, 6395-6403. 
Yokoyama, H., H. Ejiri, M. Miyazawa, S. Yamaguchi, \& Y. Hirai (2007). Asymmetric synthesis of fagomine. Tetrahedron Assym. 18, 852-856.

You, J., R. E. Cohen, \& C. M. Pickart (1999). Construct for high-level expression and low misincorporation of lysine for arginine during expression of pET-encoded eukaryotic proteins in Escherichia coli. Biotechniques 27, 950-954.

Zweckstetter, M. (2008). NMR: prediction of molecular alignment from structure using the PALES software. Nat. Protoc. 3, 679-690.

Zweckstetter, M. \& A. Bax (2000). Prediction of sterically induced alignment in a dilute liquid crystalline phase: aid to protein structure determination by NMR. $J$. Am. Chem. Soc. 122, 3791-3792. 



\section{Abbildungsverzeichnis}

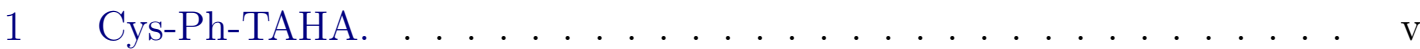

1.1 Schematische Darstellung von Ubiquitin . . . . . . . . . . . . . . . 3

1.2 Histogramme der Literaturstellen mit dem Thema „Ubiquitin“ . . . . 4

1.3 MTSSL-Tag zur paramagnetischen Markierung von Proteinen. . . . . 5

1.4 Schematische Darstellung der Ausrichtung paramagnetischer Moleküle entlang des Magnetfeldes . . . . . . . . . . . . . . 6

2.1 Definition des Winkels $\theta$ zwischen dem Interspinvektor $\vec{R}$ und dem Vektor des statischen Magnetfeldes $\vec{B}$. . . . . . . . . . . . . 10

2.2 Darstellung der molekularen Reorientierung im Laborkoordinatensystem und im Molekülkoordinatensystem . . . . . . . . . . . . . . . . 12

2.3 Graphische Darstellung des Wahrscheinlichkeitstensors P im Molekülkoordinatensystem . . . . . . . . . . . . . . . . 14

2.4 Graphische Darstellung verschiedener Alignmenttensoren . . . . . . . 15

2.5 Definition der Polarwinkel $\vartheta$ und $\varphi$ für einen beliebigen internuklearen

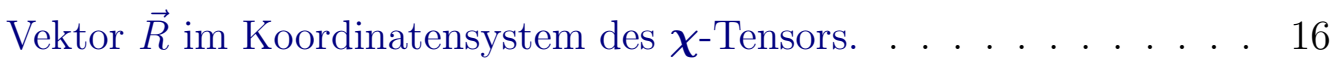

2.6 Definition der Polarwinkel $\Theta$ und $\Phi$ im Koordinatensystem des

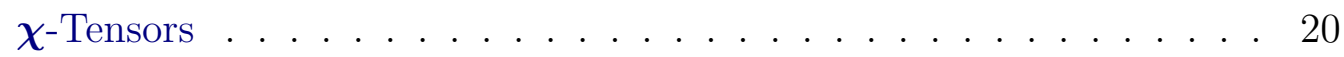

2.7 Darstellung der PCSs-Isoflächen . . . . . . . . . . . . . . . . . . 21

2.8 Experimenteller und berechneter Verlauf der $\Delta \chi_{a x}$-Werte für die Reihe der Lanthanoide . . . . . . . . . . . . . . . . . . 22

$3.1 S$-(2-Pyridylthio)cysteaminyl-EDTA . . . . . . . . . . 25

3.2 Übersicht der EDTA-basierten Tags . . . . . . . . . . . . . . . . . . 26 
3.3 Schematische Darstellung der diastereomeren Komplexbildung, ausgehend vom achiralen EDTA-Derivat . . . . . . . . . . . 27

3.4 Points-on-a-sphere-Modell der beiden enantiomeren DTPA-Konformationen im Lanthanoidkomplex . . . . . . . . . . . . . . 28

3.5 Der DTPA-basierte CLaNP-1-Tag . . . . . . . . . . . . . . . 28

3.6 Die DOTA-basierten Tags CLaNP-3 und CLaNP-5 . . . . . . . . . . 29

3.7 Der DOTA-basierte DOTA-M8-Tag . . . . . . . . . . . . . 30

3.8 Übersicht über die DPA-basierten Tags . . . . . . . . . . . . . . . . . 31

3.9 Ellman's Reagenz DTNB zur Verknüpfung thiolhaltiger Verbindungen an Cysteinseitenketten . . . . . . . . . . . . . . . . . 32

3.10 Struktur der TAHA-Liganden. . . . . . . . . . . . . . . . 34

4.1 Zielmolekül der ersten Synthesen: Cys-TAHA. . . . . . . . . . . . . . 37

4.2 Syntheseansatz ausgehend von Pentaerythrit. . . . . . . . . . . . . 39

4.3 Erster Syntheseansatz ausgehend von Pentaerythritol-tribromid . . . 40

4.4 Zweiter Syntheseansatz ausgehend von Pentaerythritol-tribromid . . . 42

4.5 Strukturvorhersage von $7 \ldots \ldots$. . . . . . . . . . . . . 43

4.6 Mechanismus der Fragmentierung von 18 zum Acrylat . . . . . . . . 44

4.7 Dritter Syntheseansatz ausgehend von Pentaerythritol-tribromid . . . 45

4.8 Neues Zielmolekül Cys-Ph-TAHA . . . . . . . . . . . . . . . . . 46

4.9 Syntheseschema von Cys-Ph-TAHA . . . . . . . . . . . . . . . . . 47

4.10 Reaktionsschema der Hydroxycarbonylierung . . . . . . . . . . . . . . 48

4.11 Chromatogramm von HPLC-gereinigtem Cys-Ph-TAHA . . . . . . . 49

4.12 Massenspektrum von ${ }^{15} \mathrm{~N}-$ markiertem T12C Tm . . . . . . . . . . . 51

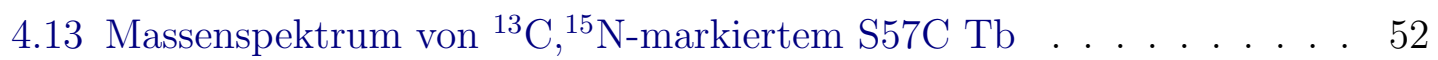

4.14 Vergleich der ${ }^{1} \mathrm{H}-$ Spektren von Cys-Ph-TAHA-getaggtem T12C, be-

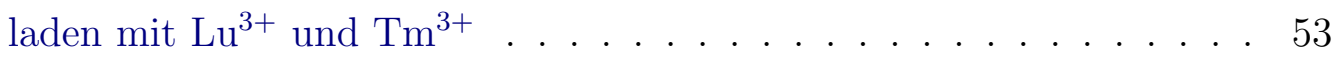

4.15 Überlagerung der ${ }^{1} \mathrm{H},{ }^{15} \mathrm{~N}-\mathrm{HSQC}$-Spektren von ungetaggtem S57C und $\mathrm{S} 57 \mathrm{C} \mathrm{Lu} \ldots \ldots \ldots \ldots . \ldots \ldots$

5.1 Überlagerung der ${ }^{1} \mathrm{H},{ }^{15} \mathrm{~N}-\mathrm{HSQC}-$ Spektren von T12C Tb und T12C Lu 56

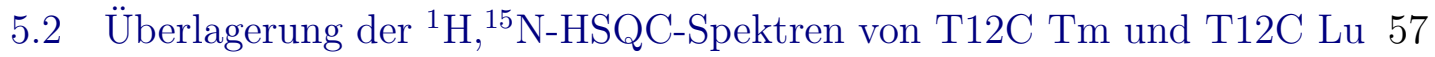


5.3 Darstellung der Aminosäurereste, deren Signale bei T12C Tb durch PRE nicht mehr detektierbar waren . . . . . . . . . . . 58

5.4 Auftragungen der experimentellen und berechneten PCSs von T12C Tb und T12C Tm gegen die Aminosäurereste . . . . . . . . . . . . . 59

5.5 Schematische Darstellung der berechneten Metallpositionen von $\mathrm{Tb}^{3+}$ und $\mathrm{Tm}^{3+}$ zusammen mit der T12C-Struktur . . . . . . . . . . . . 60

5.6 Überlagerung der ${ }^{1} \mathrm{H},{ }^{15} \mathrm{~N}-\mathrm{HSQC}-S p e k t r e n$ von S57C Tb und S57C Lu 62

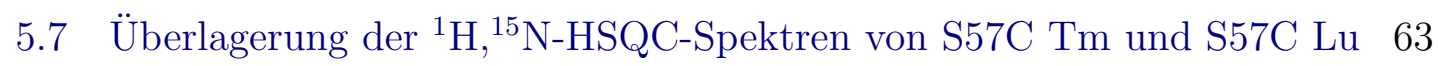

5.8 Darstellung der Aminosäurereste, deren Signale bei S57C Tm durch PRE nicht mehr detektierbar waren . . . . . . . . . . . . 64

5.9 Schematische Darstellung der berechneten Metallpositionen von $\mathrm{Tb}^{3+}$ und $\mathrm{Tm}^{3+}$ zusammen mit der S57C-Struktur . . . . . . . . . . . 65

5.10 Auftragungen der experimentellen und berechneten PCSs von S57C Tb und S57C Tm gegen die Aminosäurereste . . . . . . . . . . . . 66

5.11 Ausschnitte aus den ${ }^{1} \mathrm{H},{ }^{15} \mathrm{~N}-\mathrm{HSQC}-S p e k t r e n$ von T12C Tm bei verschiedenen Temperaturen auf einem niedrigen Contourlevel . . . . . . 68

5.12 Ausschnitt aus den überlagerten ${ }^{1} \mathrm{H},{ }^{15} \mathrm{~N}-\mathrm{HSQC}-S p e k t r e n$ von T12C Tm bei verschiedenen Temperaturen $(278 \mathrm{~K}-315 \mathrm{~K})$. . . . . . . . . 71

5.13 Darstellung der berechneten Metallpositionen in T12C Tm bei unterschiedlichen Temperaturen . . . . . . . . . . . . . 72

5.14 Auftragungen der experimentellen PCSs von T12C Tm gegen die Aminosäurereste bei verschiedenen Temperaturen . . . . . . . . . 73

5.15 Ausschnitt des IPAP-HSQC-Spektrums von T12C Tb . . . . . . . . . 74

5.16 Auftragung der experimentellen gegen die berechneten RDCs . . . . . 77

5.17 T12C Tb: Vergleich der PCS- und RDC-abgeleiteten Tensoren . . . . 79

5.18 Schematischer Vergleich zwischen PCSs und RDCs: Vergleich der Metall-Kern-Vektoren und Interspinvektoren . . . . . . . . . . . 80

5.19 Korrelationsdiagramm der experimentellen PCSs, aufgetragen gegen die im RDC-Tensor zurück berechneten Werte . . . . . . . . . . . . 82

5.20 Korrelationsdiagramm der experimentellen RDCs, aufgetragen gegen die im PCS-Tensor zurück berechneten Werte . . . . . . . . . . . 83 
5.21 Berechnete Metallpositionen aus den PCSs für T12C Tb, T12C Tm, sowie aus der gleichzeitigen Minimierung von PCSs und RDCs . . . . 86

5.22 T12C Tb: Darstellung der Metall-Kern-Vektoren der PCSs als Vektorschar, sowie deren Verteilungen auf einer Kugeloberfläche . . . . . 87

5.23 Vergleich der RDC-Tensoren mit den neu berechneten PCS-Tensoren 88

5.24 Korrelationsdiagramm der experimentellen ${ }^{1} \mathrm{H}$ - und ${ }^{15} \mathrm{~N}-\mathrm{PCSs}$ beider Cys-Ph-TAHA-getaggten Ubiquitinmutanten, aufgetragen gegen die mit den RDCs zusammen berechneten Werte . . . . . . . . . . . . . 90

5.25 Korrelationsdiagramm der experimentellen RDCs beider Cys-Ph-TAHAgetaggten Ubiquitinmutanten, aufgetragen gegen die mit den PCSs zusammen berechneten Werte . . . . . . . . . . . . . . . . 91

5.26 Auftragungen der Saupematrixelemente von T12C Tb und T12C Tm, die aus den unterschiedlichen Tensorberechnungen erhalten wurden . 93

6.1 Schematische Darstellung der Korrelationen im HNCA . . . . . . . . 101

6.2 Schematische Darstellung der Prozessierung des IPAP-HSQC . . . . . 102

6.3 Gradient der HPLC-Trennung von Cys-Ph-TAHA . . . . . . . . . . . 114

$7.1{ }^{1} \mathrm{H},{ }^{15} \mathrm{~N}-\mathrm{HSQC}-S p e k t r u m$ der isotropen Referenzprobe T12C Lu . . . . 144

$7.2{ }^{1} \mathrm{H},{ }^{15} \mathrm{~N}-\mathrm{HSQC}-S p e k t r u m$ der isotropen Referenzprobe S57C Lu . . . . 145

7.3 Berechnete Metallpositionen aus den PCSs für S57C Tb und S57C Tm, sowie aus der gleichzeitigen Minimierung von PCSs und RDCs . . . . 146

7.4 T12C Tb: Darstellung der erhaltenen Tensoren . . . . . . . . . . . . . 147

7.5 T12C Tm: Darstellung der erhaltenen Tensoren . . . . . . . . . . . 148

7.6 S57C Tb: Darstellung der erhaltenen Tensoren . . . . . . . . . . . . 149

7.7 S57C Tm: Darstellung der erhaltenen Tensoren . . . . . . . . . . . 150 


\section{Tabellenverzeichnis}

3.1 Wassermoleküle in der inneren Koordinationssphäre des TAHA Liganden . . . . . . . . . . . . . . . . . . . 35

5.1 Zusammenfassung der Ergebnisse der PCS Analysen . . . . . . . . . 67

5.2 T12C Tm: Bestimmung der prozentualen Verteilungen der Peakintensitäten bei verschiedenen Temperaturen . . . . . . . . . . . . . 69

5.3 Zusammenfassung der Ergebnisse der RDC Analysen . . . . . . . . . 76

5.4 Übersicht über die berechneten Tensoren aus den PCSs und RDCs der vier anisotropen Proben . . . . . . . . . . . . . . . 79

5.5 Ergebnisse der Berechnungen der PCSs im RDC-Tensor, sowie der Berechnung der RDCs im PCS-Tensor . . . . . . . . . . . . . . 81

5.6 Übersicht über die erhaltenen Tensoren . . . . . . . . . . . . . . . . . 85

5.7 Übersicht über die Mittelwerte und Standardabweichungen der Saupematrixelemente von $\mathrm{T} 12 \mathrm{C} \mathrm{Tb}$ und $\mathrm{T} 12 \mathrm{C}$ Tm, die aus 100 Berechnungen mit jeweils $80 \%$ der Daten erhalten wurden . . . . . . . . . . 92

7.1 Übersicht der 20 Standardaminosäuren im Drei- und Einbuchstabenkode. . . . . . . . . . . . . . . . . . . . 143

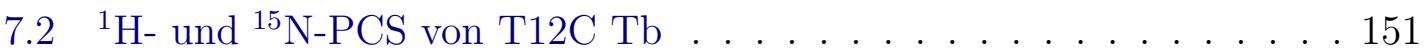

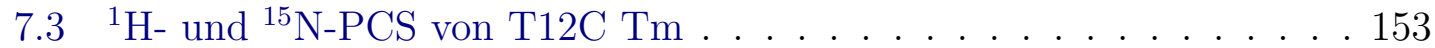

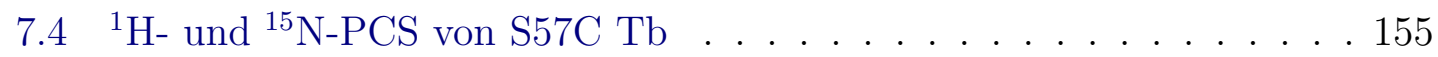

$7.5{ }^{1} \mathrm{H}-$ und ${ }^{15} \mathrm{~N}-\mathrm{PCS}$ von S57C Tm . . . . . . . . . . . . . . . . 157

7.6 Chemische Verschiebungen in ${ }^{1} \mathrm{H}$ und ${ }^{15} \mathrm{~N}$ der Hauptpeaks und zusätzlichen Signalsätze von zehn Aminosäureresten bei verschiedenen Tem-

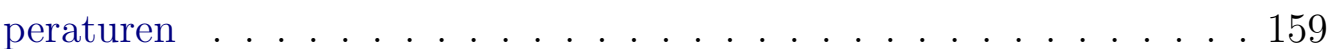


7.7 Prozentuale Verteilungen der Hauptpeaks und der zusätzlichen Signalsätze von zehn Aminosäureresten bei verschiedenen Temperaturen 161

7.8 T12C Lu: Chemische Verschiebungen $\left({ }^{1} \mathrm{H}\right.$ und $\left.{ }^{15} \mathrm{~N}\right)$ der Amidsignale des Proteinrückgrats bei unterschiedlichen Temperaturen . . . . . . . 163

7.9 T12C Tm: Chemische Verschiebungen $\left({ }^{1} \mathrm{H}\right.$ und $\left.{ }^{15} \mathrm{~N}\right)$ der Amidsignale des Proteinrückgrats bei unterschiedlichen Temperaturen . . . . . . . 165

7.10 T12C Tm: PCSs $\left({ }^{1} \mathrm{H}\right.$ und $\left.{ }^{15} \mathrm{~N}\right)$ der Amidsignale des Proteinrückgrats in Abhängigkeit von der Temperatur . . . . . . . . . . . . . . . 167

7.11 T12C: ${ }^{1} J$ N-H Kopplungen des Proteinrückgrats der isotropen und anisotropen Proben und deren experimentelle und zurück berechnete RDCs . . . . . . . . . . . . . . . . . . 169

7.12 S57C: ${ }^{1} J$ N-H Kopplungen des Proteinrückgrats der isotropen und anisotropen Proben und deren experimentelle und zurück berechnete

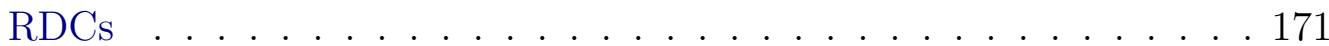

$7.13{ }^{1} \mathrm{H},{ }^{15} \mathrm{~N}-\mathrm{PCS}$ von T12C Tb und T12C Tm, berechnet im RDC-Tensor und deren Abweichungen $\Delta$ von den experimentellen Werten . . . . . 173

$7.14{ }^{1} \mathrm{H},{ }^{15} \mathrm{~N}-\mathrm{PCS}$ von S57C Tb und S57C Tm, berechnet im RDC-Tensor und deren Abweichungen $\Delta$ von den experimentellen Werten . . . . 175

7.15 Werte der im jeweiligen PCS-Tensor berechneten RDCs und deren Differenz $\Delta$ zum experimentellen RDC der vier anisotropen Proben . 177

7.16 Übersicht über die Saupe-Matrixelemente der anisotropen Proben . . 179

$7.17{ }^{1} \mathrm{H},{ }^{15} \mathrm{~N}-\mathrm{PCS}$ von T12C Tb und T12C Tm, zusammen mit den RDCs minimiert, sowie deren Abweichungen $\Delta$ von den experimentellen

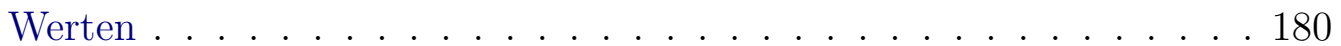

$7.18{ }^{1} \mathrm{H},{ }^{15} \mathrm{~N}-\mathrm{PCS}$ von S57C Tb und S57C Tm, zusammen mit den RDCs minimiert, sowie deren Abweichungen $\Delta$ von den experimentellen Werten . . . . . . . . . . . . . . . . 182

7.19 Werte der berechneten RDCs, zusammen mit den PCSs minimiert, sowie deren Differenz $\Delta$ zu den experimentellen Werten aller vier anisotropen Proben . . . . . . . . . . . . . . . . . . . . . 184 


\section{Abkürzungsverzeichnis}

\begin{tabular}{|c|c|}
\hline 3MDPA & 3-Mercaptopyridin-2,6-dicarbonsäure \\
\hline 4MMDPA & 4-(Mercaptomethyl)pyridin-2,6-dicarbonsäure \\
\hline abs. & absolut \\
\hline$\ddot{A} q$. & Äquivalente \\
\hline AS & Aminosäure \\
\hline ber. & berechnet \\
\hline $\mathrm{Bn}$ & Benzyl \\
\hline Boc & tert-Butyloxycarbonyl \\
\hline br & breit \\
\hline CLaNP & Caged Lanthanide NMR Probe \\
\hline COSY & Correlation Spectroscopy \\
\hline Cys-Ph-TAHA & Cysteinyl-Phenyl-TAHA \\
\hline Cys-TAHA & Cysteinyl-TAHA \\
\hline $\mathrm{d}$ & Dublett \\
\hline DNA & 2'-Desoxyribonukleinsäure \\
\hline $\mathrm{DC}$ & Dünnschichtchromatographie \\
\hline DIPEA & Diisopropylethylamin \\
\hline DIS & Dysprosium Induced Shift \\
\hline DMAP & 4-(Dimethylamino)-pyridin \\
\hline $\mathrm{DMF}$ & Dimethylformamid \\
\hline DMSO & Dimethylsulfoxid \\
\hline DOTA & 1,4,7,10-Tetraazacyclododecan-1,4,7,10-tetraessigsäure \\
\hline $\mathrm{DPA}$ & Dipicolinsäure \\
\hline dppf & 1,1'-Bis(diphenylphosphino)ferrocen \\
\hline DTNB & 5,5'-Dithiobis-(2-nitrobenzoesäure) \\
\hline DTPA & Diethylentriaminpentaessigsäure \\
\hline EDTA & Ethylendiamintetraessigsäure \\
\hline
\end{tabular}


EROS Ensemble Refinement with Orientational Restraints

ESI-MS Elektrospray Ionisation Mass Spectrometry

ESR Elektronenspinresonanz

Et-TAHA Ethyl-TAHA

exp. experimentell

gef. gefunden

GFP Green Fluorescent Protein

HATU O-(7-Azabenzotriazol-1-yl)- $N, N, N^{\prime}, N^{\prime}$-tetramethyluronium-

Hexafluorophosphat

HPLC High Performance Liquid Chromatography

HSQC Heteronuclear Single Quantum Coherence

IPAP In-phase/ Antiphase

LC-MS Liquid Chromatography-Mass Spectrometry

MAS Magic Angle Spinning

MOPS 3-(N-Morpholino)propansulfonsäure

MTSSL (1-Oxyl-2,2,5,5-tetramethyl-1H-3-pyrrolin-3-methyl)methanthiosulfonat Spin Label

MWCO Molecular Weight Cutoff

neg. negativ

NMO N-Methylmorpholin- $N$-oxid

NMR Nuclear Magnetic Resonance

NOESY Nuclear Overhauser Enhancement Spectroscopy

NTA Nitrilotriessigsäure

p.a. pro analysis

PCS Pseudocontact Shift

PDB Protein Data Bank

PES Polyethersulfon

Ph-TAHA Phenyl-TAHA

pos. positiv

PRE Paramagnetic Relaxation Enhancement 
q

rcf

$\mathrm{RDC}$

RNA

$\mathrm{S}$

S57C

S57C Lu

$\mathrm{S} 57 \mathrm{C} \mathrm{Tb}$

S57C Tm

t

$\mathrm{T} 12 \mathrm{C}$

T12C Lu

$\mathrm{T} 12 \mathrm{C} \mathrm{Tb}$

T12C Tm

TAHA

TAME-Hex

TBAF

TBDPS-Cl

TCEP

TFA

THF

TMSE

Tos

TPAP

TPPI
Quartett

Relative Centrifugal Force

Residual Dipolar Coupling

Ribonukleinsäure

Singulett

Ubiquitinmutante Serin57 $\rightarrow$ Cystein57

S57C getaggt mit Cys-Ph-TAHA, beladen mit $\mathrm{Lu}^{3+}$ S57C getaggt mit Cys-Ph-TAHA, beladen mit $\mathrm{Tb}^{3+}$ S57C getaggt mit Cys-Ph-TAHA, beladen mit $\mathrm{Tm}^{3+}$ Triplett

Ubiquitinmutante Threonin12 $\rightarrow$ Cystein12

T12C getaggt mit Cys-Ph-TAHA, beladen mit $\mathrm{Lu}^{3+}$ T12C getaggt mit Cys-Ph-TAHA, beladen mit $\mathrm{Tb}^{3+}$ T12C getaggt mit Cys-Ph-TAHA, beladen mit $\mathrm{Tm}^{3+}$ Triaminohexaacetat

Tris(aminomethyl)ethan- $N, N, N^{\prime}, N^{\prime}, N^{\prime \prime}, N$ "-hexaessigsäure Tetra- $N$-butylammoniumfluorid

tert-Butyldiphenylsilylethanol

Phosphorsäure-tris(2-chlorethyl)ester

Trifluoressigsäure

Tetrahydrofuran

Trimethylsilylethanol

Tosyl

Tetra- $N$-propylammoniumperruthenat

Time Proportional Phase Increment 


\section{Kapitel 7}

\section{Anhang}

Tab. 7.1: Übersicht der 20 Standardaminosäuren im Drei- und Einbuchstabenkode.

\begin{tabular}{lcc}
\hline Aminosäure & Dreibuchstabenkode & Einbuchstabenkode \\
\hline Alanin & Ala & $\mathrm{A}$ \\
Arginin & Arg & $\mathrm{R}$ \\
Asparagin & Asn & $\mathrm{N}$ \\
Aspartat & Asp & $\mathrm{D}$ \\
Cystein & Cys & $\mathrm{C}$ \\
Glutamin & Gln & $\mathrm{Q}$ \\
Glutamat & Glu & $\mathrm{E}$ \\
Glycin & Gly & $\mathrm{G}$ \\
Histidin & His & $\mathrm{H}$ \\
Isoleucin & Ile & $\mathrm{I}$ \\
Leucin & Leu & $\mathrm{L}$ \\
Lysin & Lys & $\mathrm{K}$ \\
Methionin & Met & $\mathrm{M}$ \\
Phenylalanin & Phe & $\mathrm{F}$ \\
Prolin & Pro & $\mathrm{P}$ \\
Serin & Ser & $\mathrm{S}$ \\
Threonin & Thr & $\mathrm{T}$ \\
Tryptophan & Trp & $\mathrm{W}$ \\
Tyrosin & Tyr & $\mathrm{Y}$ \\
Valin & Val & $\mathrm{V}$ \\
\hline & & \\
\hline
\end{tabular}




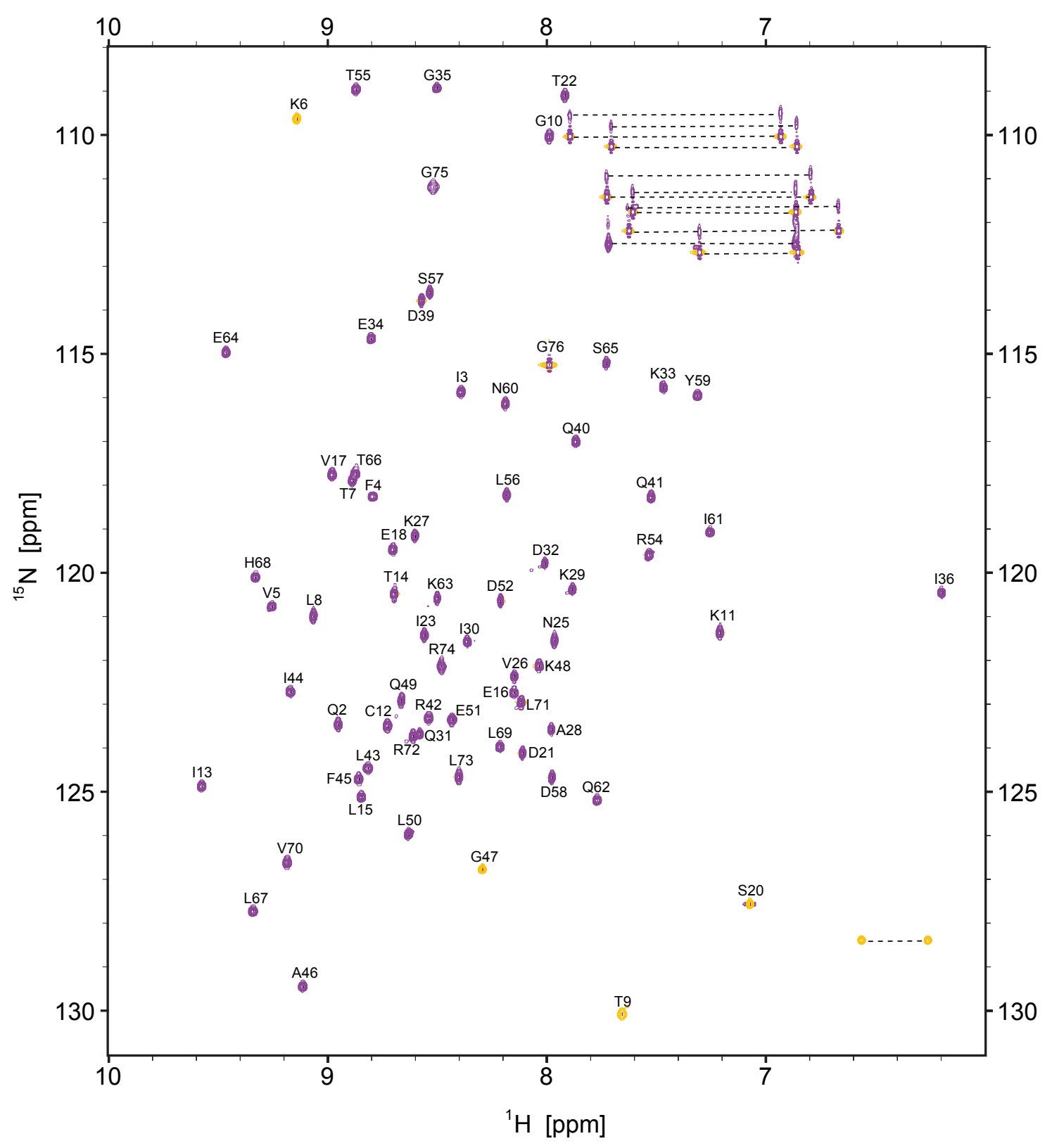

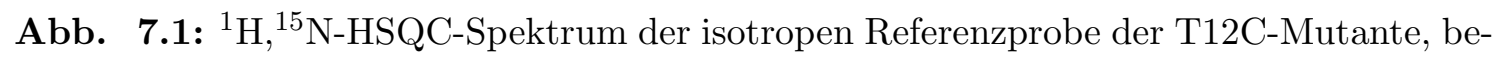
laden mit $\mathrm{Lu}^{3+}$ (violett). Gefaltete Peaks (States-TPPI) sind orange dargestellt. Signale von Seitenketten sind durch unterbrochene Linien angezeigt. Eine vollständige Liste der chemischen Verschiebungen ist in der Tabelle 7.2 angegeben. 


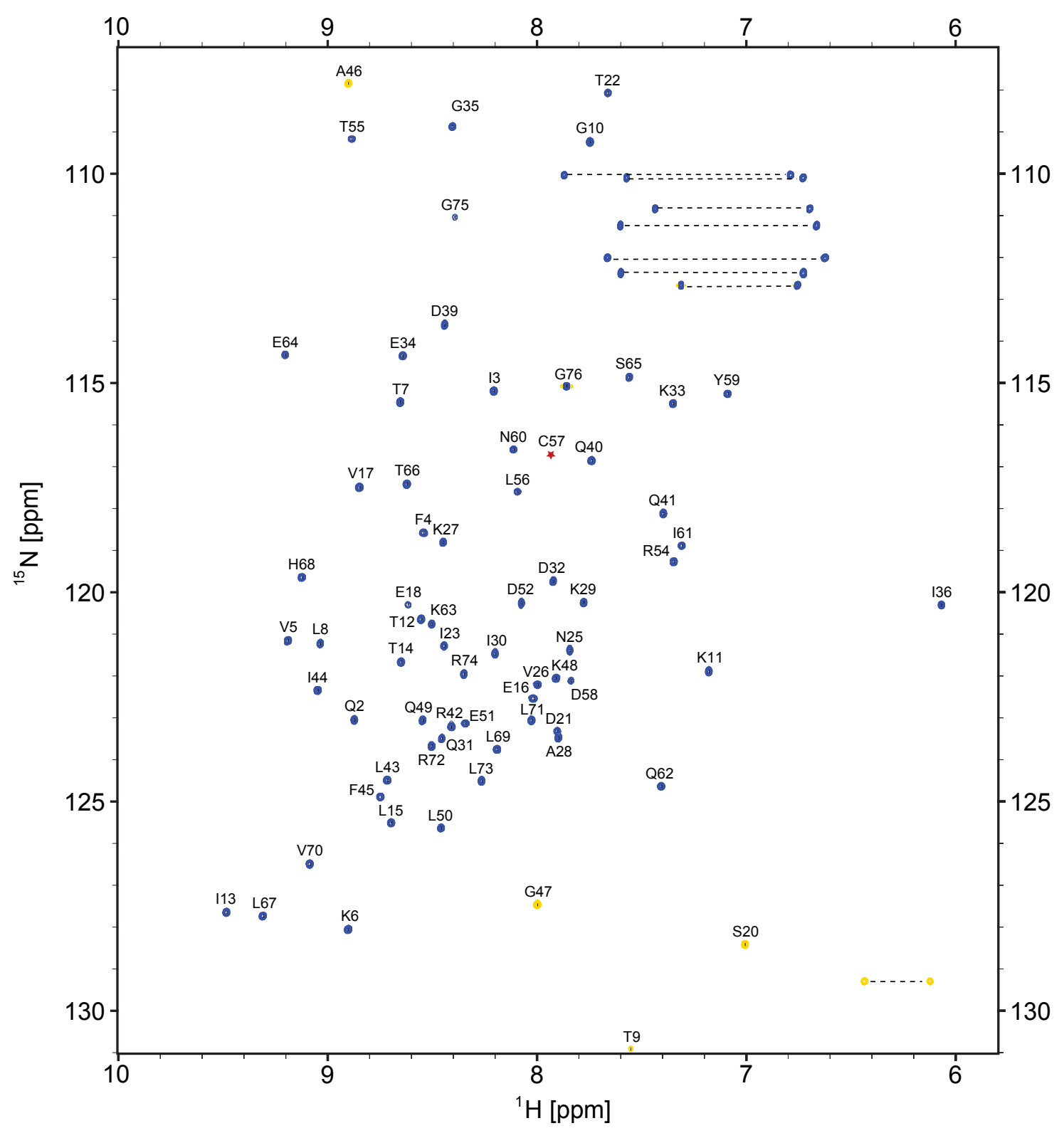

Abb. 7.2: ${ }^{1} \mathrm{H},{ }^{15} \mathrm{~N}-\mathrm{HSQC}-$ Spektrum der isotropen Referenzprobe der S57C-Mutante, beladen mit $\mathrm{Lu}^{3+}$ (blau). Gefaltete Peaks (States-TPPI) sind gelb dargestellt. Der Peak von C57 ist schwach und durch einen roten Stern angedeutet. Signale von Seitenketten sind durch unterbrochene Linien angezeigt. Eine vollständige Liste der chemischen Verschiebungen ist in der Tabelle 7.4 angegeben. 


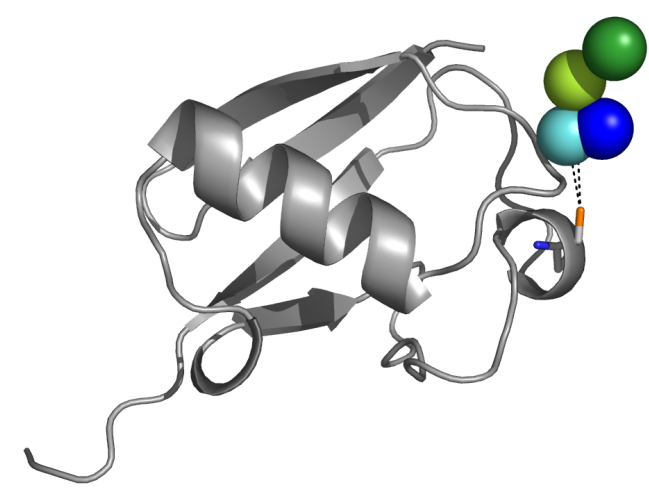

Abb. 7.3: Darstellung der Metallpositionen, berechnet aus den PCSs für S57C Tb (dunkelgrün) und S57C Tm (dunkelblau), sowie aus der gleichzeitigen Minimierung von PCSs und RDCs: S57C Tb (hellgrün), S57C Tm (hellblau). 

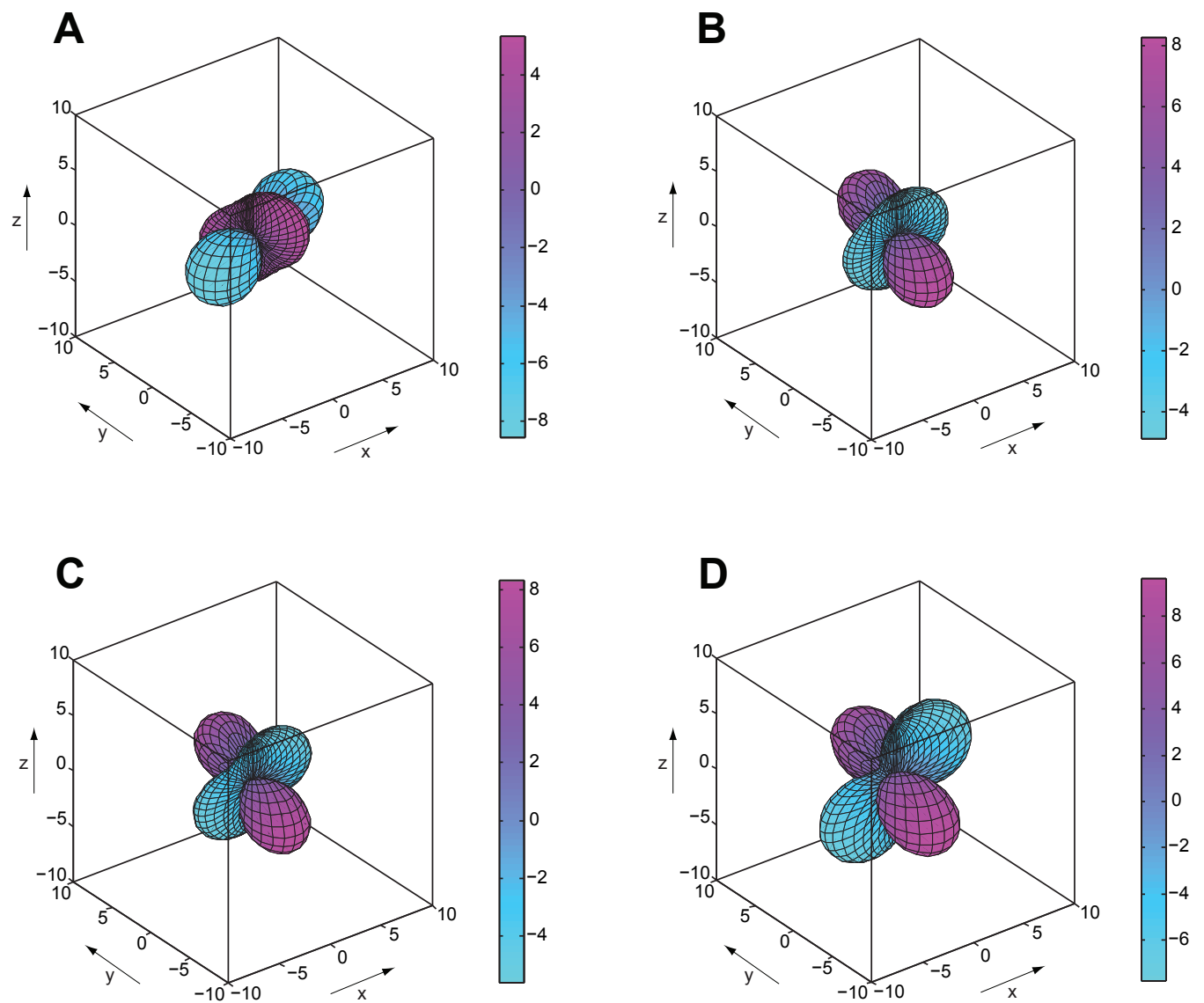

Abb. 7.4: T12C Tb: Darstellung der erhaltenen Tensoren $\left[10^{-4}\right]$. Die Tensoren sind wie folgt zugeordnet: PCS-Tensor (A), RDC-Tensor (B), Tensor der gleichzeitigen Minimierung von PCSs und RDCs (C), Tensor auf Basis der RDC-abgeleiteten Metallposition mit anschließender Minimierung der PCSs (D). Die zu Grunde liegenden Werte finden sich in den Tabellen 5.6 und 7.16. 

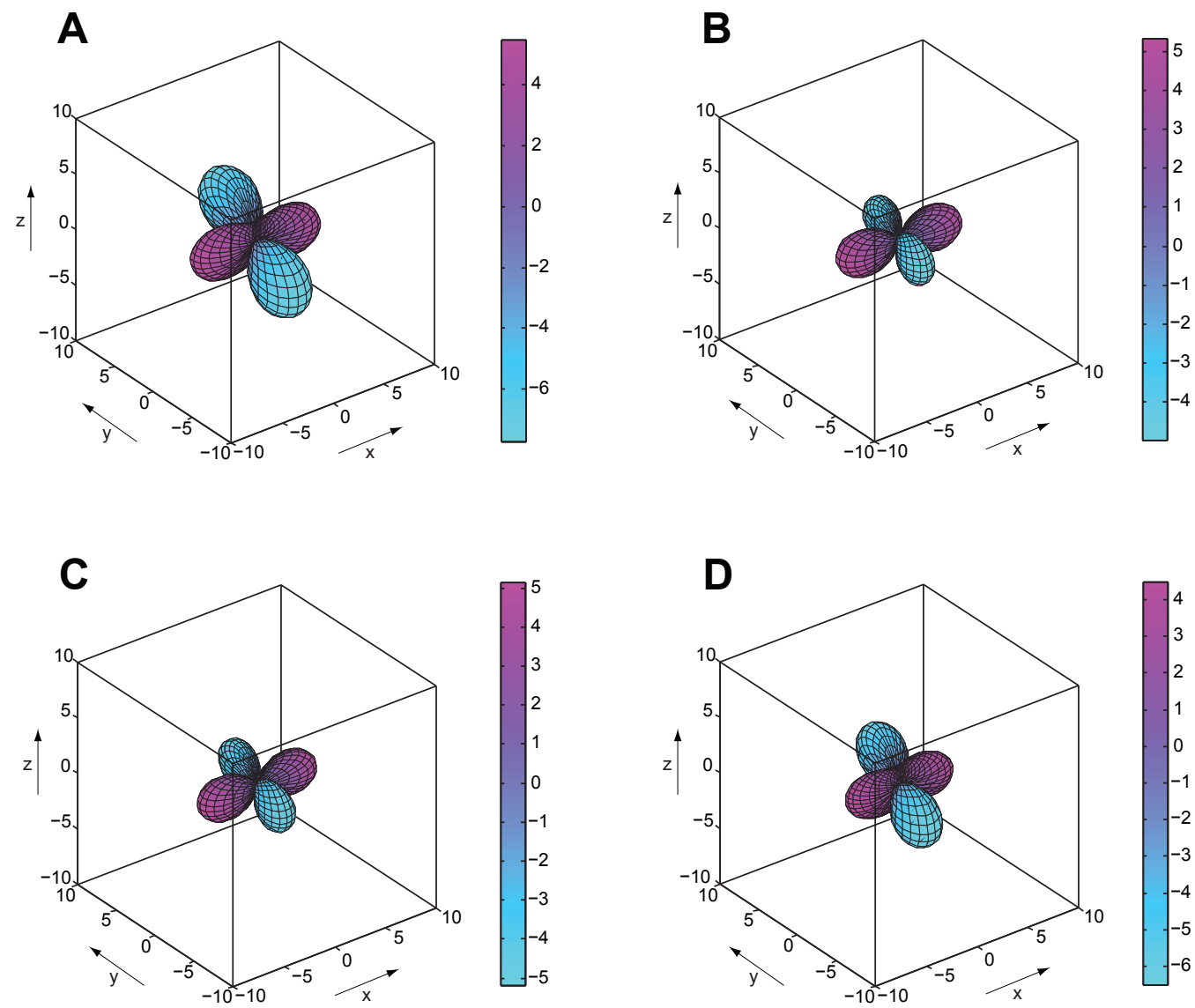

Abb. 7.5: T12C Tm: Darstellung der erhaltenen Tensoren $\left[10^{-4}\right]$. Die Tensoren sind wie folgt zugeordnet: PCS-Tensor (A), RDC-Tensor (B), Tensor der gleichzeitigen Minimierung von PCSs und RDCs (C), Tensor auf Basis der RDC-abgeleiteten Metallposition mit anschließender Minimierung der PCSs (D). Die zu Grunde liegenden Werte finden sich in den Tabellen 5.6 und 7.16. 

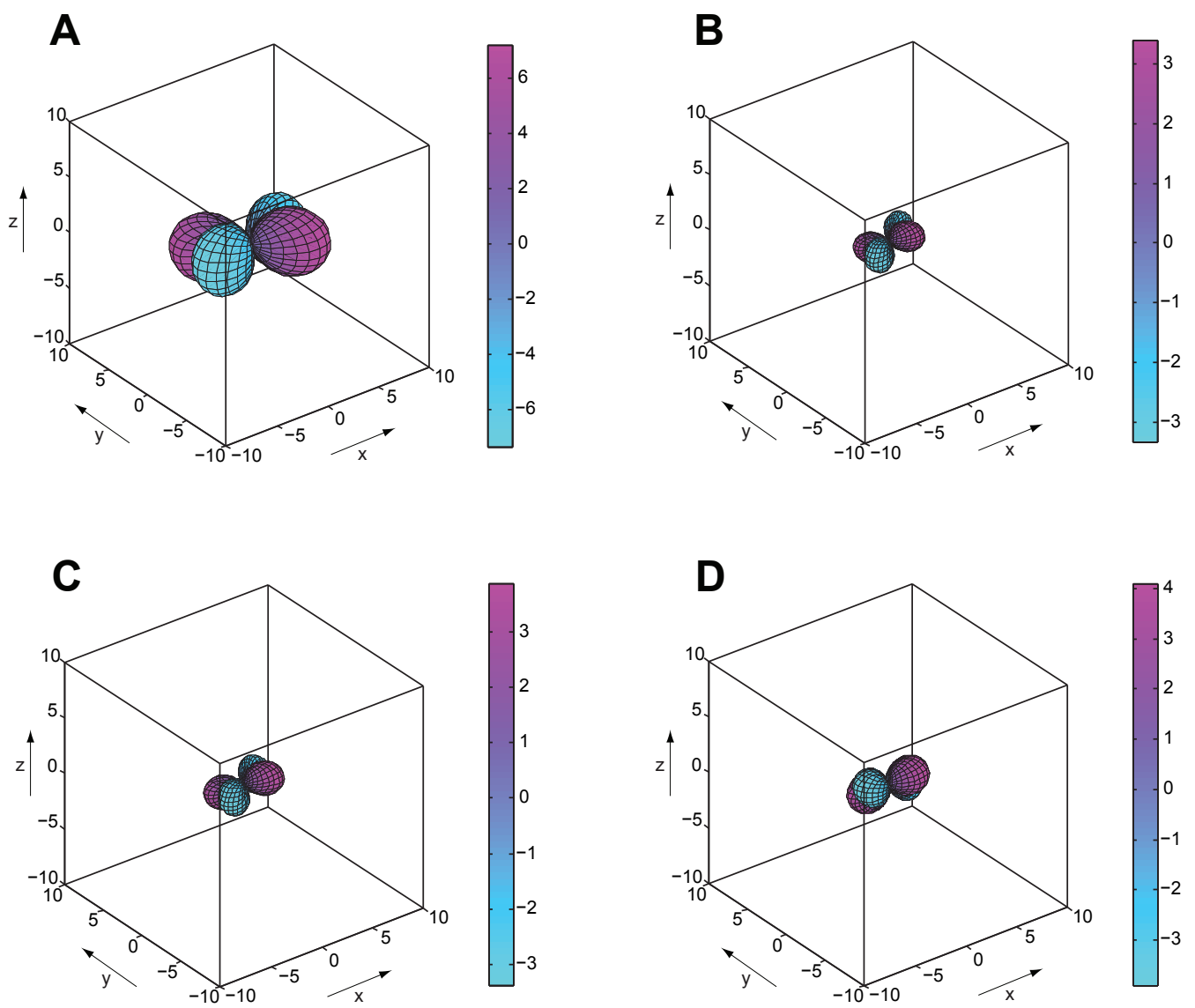

Abb. 7.6: S57C Tb: Darstellung der erhaltenen Tensoren $\left[10^{-4}\right]$. Die Tensoren sind wie folgt zugeordnet: PCS-Tensor (A), RDC-Tensor (B), Tensor der gleichzeitigen Minimierung von PCSs und RDCs (C), Tensor auf Basis der RDC-abgeleiteten Metallposition mit anschließender Minimierung der PCSs (D). Die zu Grunde liegenden Werte finden sich in den Tabellen 5.6 und 7.16. 

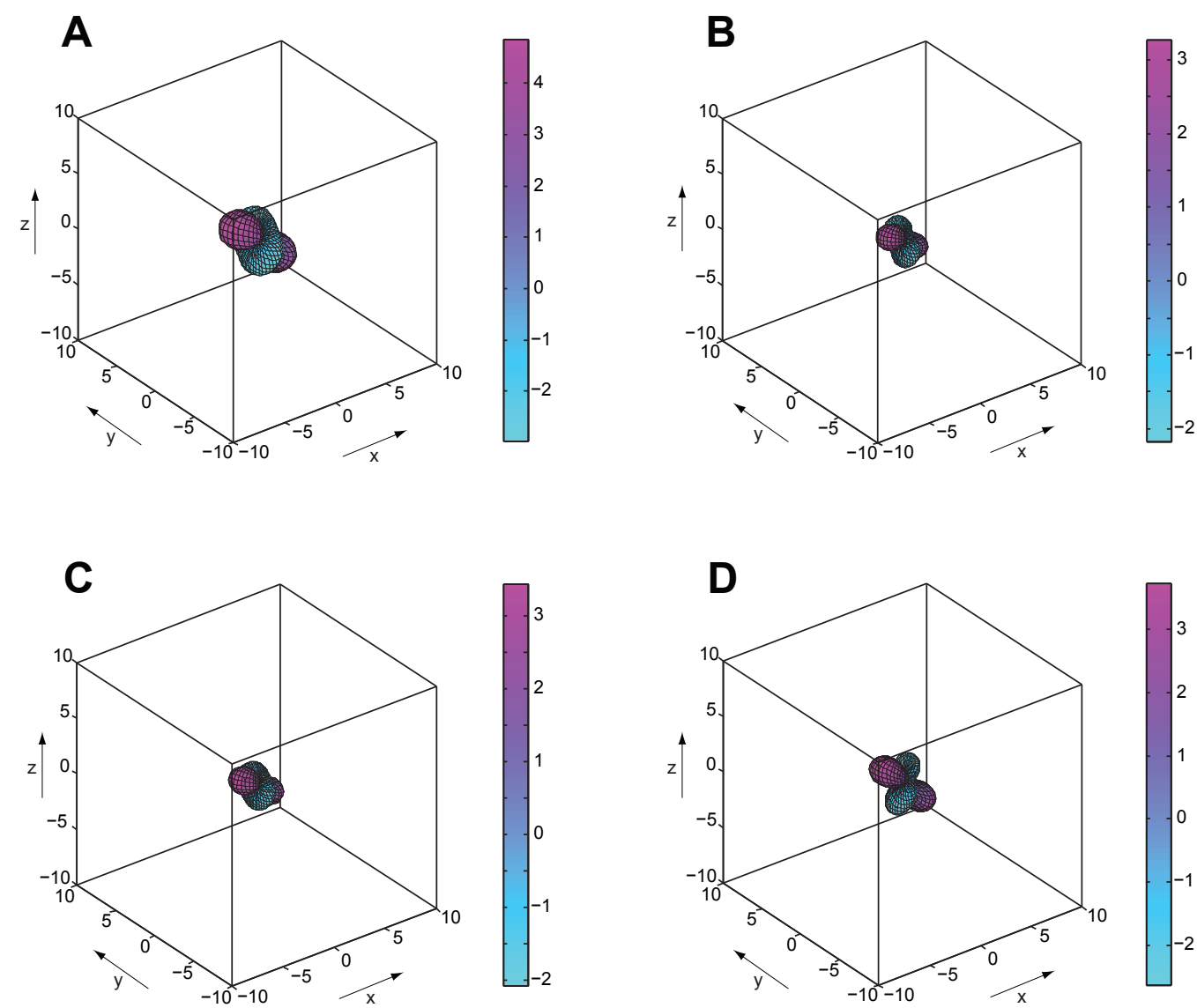

Abb. 7.7: S57C Tm: Darstellung der erhaltenen Tensoren $\left[10^{-4}\right]$. Die Tensoren sind wie folgt zugeordnet: PCS-Tensor (A), RDC-Tensor (B), Tensor der gleichzeitigen Minimierung von PCSs und RDCs (C), Tensor auf Basis der RDC-abgeleiteten Metallposition mit anschließender Minimierung der PCSs (D). Die zu Grunde liegenden Werte finden sich in den Tabellen 5.6 und 7.16. 
Tab. 7.2: 59 Pseudokontaktverschiebungen von T12C Tb [ppm]. Als diamagnetische Referenzprobe wurde T12C Lu verwendet. Gegeben sind die zugrunde liegenden chemischen Verschiebungen, sowie die experimentellen und berechneten PCSs und deren Differenz $\Delta$ für ${ }^{1} \mathrm{H}$ und ${ }^{15} \mathrm{~N}$. Die Daten wurden bei $298 \mathrm{~K}$ aufgenommen.

\begin{tabular}{|c|c|c|c|c|c|c|c|c|c|c|}
\hline \multirow[b]{2}{*}{$\mathrm{AS}$} & \multicolumn{2}{|c|}{ Terbium } & \multicolumn{2}{|c|}{ Lutetium } & \multicolumn{3}{|c|}{$\operatorname{PCS}{ }^{1} \mathrm{H}$} & \multicolumn{3}{|c|}{$\operatorname{PCS}{ }^{15} \mathrm{~N}$} \\
\hline & ${ }^{1} \mathrm{H}$ & ${ }^{15} \mathrm{~N}$ & ${ }^{1} \mathrm{H}$ & ${ }^{15} \mathrm{~N}$ & $\exp$ & ber & $\Delta$ & $\exp$ & ber & $\Delta$ \\
\hline $\mathrm{Q} 2$ & 9.187 & 123.653 & 8.951 & 123.475 & 0.236 & 0.261 & -0.025 & 0.178 & 0.219 & -0.041 \\
\hline I3 & 8.616 & 116.103 & 8.391 & 115.876 & 0.225 & 0.223 & 0.002 & 0.227 & 0.183 & 0.044 \\
\hline $\mathrm{F} 4$ & 8.799 & 118.436 & 8.793 & 118.262 & 0.006 & -0.308 & 0.314 & 0.174 & 0.059 & 0.115 \\
\hline V5 & 9.655 & 121.027 & 9.254 & 120.774 & 0.401 & 0.551 & -0.150 & 0.253 & 0.248 & 0.005 \\
\hline $\mathrm{K} 6$ & - & - & 9.113 & 129.453 & - & - & - & - & - & - \\
\hline $\mathrm{T} 7$ & 10.127 & 119.039 & 8.887 & 117.897 & 1.240 & 1.322 & -0.082 & 1.142 & 1.016 & 0.126 \\
\hline L8 & 9.837 & 122.067 & 9.065 & 120.995 & 0.772 & 0.624 & 0.148 & 1.072 & 0.798 & 0.274 \\
\hline T9 & 8.657 & 107.144 & 7.657 & 106.085 & 1.000 & 0.923 & 0.077 & 1.059 & 1.001 & 0.058 \\
\hline G10 & 9.732 & 111.644 & 7.989 & 110.048 & 1.743 & 1.587 & 0.156 & 1.596 & 1.643 & -0.047 \\
\hline K11 & - & - & 7.210 & 121.375 & - & - & - & - & - & - \\
\hline $\mathrm{C} 12$ & - & - & 8.726 & 123.497 & - & - & - & - & - & - \\
\hline $\mathrm{I} 13$ & 10.568 & 126.161 & 9.575 & 124.879 & 0.993 & 1.110 & -0.117 & 1.282 & 1.190 & 0.092 \\
\hline $\mathrm{T} 14$ & 9.553 & 121.323 & 8.696 & 120.484 & 0.857 & 0.825 & 0.032 & 0.839 & 0.844 & -0.005 \\
\hline L15 & 9.211 & 125.494 & 8.845 & 125.132 & 0.366 & 0.423 & -0.057 & 0.362 & 0.420 & -0.058 \\
\hline E16 & 8.307 & 122.902 & 8.148 & 122.747 & 0.159 & 0.205 & -0.046 & 0.155 & 0.211 & -0.056 \\
\hline V17 & 8.998 & 117.728 & 8.979 & 117.766 & 0.019 & 0.068 & -0.049 & -0.038 & 0.051 & -0.089 \\
\hline E18 & 8.585 & 119.315 & 8.701 & 119.472 & -0.116 & -0.082 & -0.034 & -0.157 & -0.070 & -0.087 \\
\hline $\mathrm{S} 20$ & 6.890 & 103.393 & 7.072 & 103.568 & -0.182 & -0.146 & -0.036 & -0.175 & -0.172 & -0.003 \\
\hline D21 & 7.920 & 123.907 & 8.111 & 124.124 & -0.191 & -0.170 & -0.021 & -0.217 & -0.165 & -0.052 \\
\hline $\mathrm{T} 22$ & 7.754 & 108.949 & 7.917 & 109.099 & -0.163 & -0.151 & -0.012 & -0.150 & -0.180 & 0.030 \\
\hline $\mathrm{I} 23$ & 8.307 & 121.212 & 8.559 & 121.431 & -0.252 & -0.270 & 0.018 & -0.219 & -0.259 & 0.040 \\
\hline N25 & 7.815 & 121.453 & 7.965 & 121.551 & -0.150 & -0.144 & -0.006 & -0.098 & -0.124 & 0.026 \\
\hline $\mathrm{V} 26$ & 7.988 & 122.258 & 8.148 & 122.367 & -0.160 & -0.160 & 0.000 & -0.109 & -0.128 & 0.019 \\
\hline $\mathrm{K} 27$ & 8.458 & 119.047 & 8.602 & 119.163 & -0.144 & -0.143 & -0.001 & -0.116 & -0.116 & 0.000 \\
\hline $\mathrm{A} 28$ & 7.899 & 123.546 & 7.980 & 123.583 & -0.081 & -0.060 & -0.021 & -0.037 & -0.038 & 0.001 \\
\hline K29 & 7.848 & 120.412 & 7.883 & 120.383 & -0.035 & -0.008 & -0.027 & 0.029 & 0.018 & 0.011 \\
\hline I30 & 8.371 & 121.629 & 8.363 & 121.579 & 0.008 & 0.031 & -0.023 & 0.050 & 0.068 & -0.018 \\
\hline Q31 & 8.594 & 123.730 & 8.580 & 123.688 & 0.014 & 0.053 & -0.039 & 0.042 & 0.079 & -0.037 \\
\hline D32 & 8.039 & 119.863 & 8.009 & 119.792 & 0.030 & 0.085 & -0.055 & 0.071 & 0.099 & -0.028 \\
\hline K33 & 7.561 & 115.883 & 7.468 & 115.775 & 0.093 & 0.154 & -0.061 & 0.108 & 0.165 & -0.057 \\
\hline E34 & 8.939 & 114.746 & 8.801 & 114.662 & 0.138 & 0.189 & -0.051 & 0.084 & 0.209 & -0.125 \\
\hline G35 & 8.580 & 108.964 & 8.501 & 108.935 & 0.079 & 0.148 & -0.069 & 0.029 & 0.154 & -0.125 \\
\hline I36 & 6.268 & 120.486 & 6.198 & 120.455 & 0.070 & 0.136 & -0.066 & 0.031 & 0.124 & -0.093 \\
\hline D39 & 8.503 & 113.757 & 8.572 & 113.785 & -0.069 & -0.022 & -0.047 & -0.028 & -0.035 & 0.007 \\
\hline Q40 & 7.800 & 116.955 & 7.866 & 117.011 & -0.066 & -0.009 & -0.057 & -0.056 & -0.016 & -0.040 \\
\hline Q41 & 7.428 & 118.146 & 7.524 & 118.286 & -0.096 & -0.046 & -0.050 & -0.140 & -0.039 & -0.101 \\
\hline
\end{tabular}


Tab. 7.2: Fortsetzung.

\begin{tabular}{|c|c|c|c|c|c|c|c|c|c|c|}
\hline \multirow[b]{2}{*}{$\mathrm{AS}$} & \multicolumn{2}{|c|}{ Terbium } & \multicolumn{2}{|c|}{ Lutetium } & \multicolumn{3}{|c|}{$\mathrm{PCS}{ }^{1} \mathrm{H}$} & \multicolumn{3}{|c|}{$\operatorname{PCS}{ }^{15} \mathrm{~N}$} \\
\hline & ${ }^{1} \mathrm{H}$ & ${ }^{15} \mathrm{~N}$ & ${ }^{1} \mathrm{H}$ & ${ }^{15} \mathrm{~N}$ & $\exp$ & ber & $\Delta$ & exp & ber & $\Delta$ \\
\hline $\mathrm{R} 42$ & 8.321 & 123.065 & 8.539 & 123.329 & -0.218 & -0.130 & -0.088 & -0.264 & -0.161 & -0.103 \\
\hline L43 & 8.374 & 123.977 & 8.816 & 124.468 & -0.442 & -0.396 & -0.046 & -0.491 & -0.420 & -0.071 \\
\hline I44 & 8.095 & 121.711 & 9.169 & 122.725 & -1.074 & -0.937 & -0.137 & -1.014 & -0.931 & -0.083 \\
\hline F45 & 7.507 & 123.263 & 8.859 & 124.713 & -1.352 & -1.468 & 0.116 & -1.450 & -1.688 & 0.238 \\
\hline A 46 & - & - & 9.142 & 133.633 & - & - & - & - & - & - \\
\hline G47 & - & - & 8.295 & 106.775 & - & - & - & - & - & - \\
\hline K48 & - & - & 8.034 & 122.131 & - & - & - & - & - & - \\
\hline Q49 & 8.141 & 122.341 & 8.664 & 122.923 & -0.523 & -0.697 & 0.174 & -0.582 & -0.763 & 0.181 \\
\hline L50 & 8.001 & 125.369 & 8.631 & 125.972 & -0.630 & -0.673 & 0.043 & -0.603 & -0.644 & 0.041 \\
\hline E51 & 8.065 & 122.973 & 8.432 & 123.360 & -0.367 & -0.450 & 0.083 & -0.387 & -0.424 & 0.037 \\
\hline D52 & 7.974 & 120.400 & 8.211 & 120.650 & -0.237 & -0.250 & 0.013 & -0.250 & -0.263 & 0.013 \\
\hline R54 & 7.294 & 119.331 & 7.532 & 119.598 & -0.238 & -0.280 & 0.042 & -0.267 & -0.268 & 0.001 \\
\hline $\mathrm{T} 55$ & 8.584 & 108.727 & 8.870 & 108.963 & -0.286 & -0.320 & 0.034 & -0.236 & -0.318 & 0.082 \\
\hline L56 & 7.866 & 117.873 & 8.183 & 118.229 & -0.317 & -0.341 & 0.024 & -0.356 & -0.392 & 0.036 \\
\hline $\mathrm{S} 57$ & 8.172 & 113.197 & 8.535 & 113.605 & -0.363 & -0.358 & -0.005 & -0.408 & -0.411 & 0.003 \\
\hline D58 & 7.595 & 124.312 & 7.977 & 124.679 & -0.382 & -0.406 & 0.024 & -0.367 & -0.431 & 0.064 \\
\hline Y59 & 6.765 & 115.420 & 7.312 & 115.960 & -0.547 & -0.592 & 0.045 & -0.540 & -0.618 & 0.078 \\
\hline N60 & 7.514 & 115.306 & 8.189 & 116.145 & -0.675 & -0.629 & -0.046 & -0.839 & -0.725 & -0.114 \\
\hline I61 & 6.444 & 118.083 & 7.254 & 119.080 & -0.810 & -0.766 & -0.044 & -0.997 & -0.860 & -0.137 \\
\hline Q62 & 6.545 & 124.197 & 7.770 & 125.201 & -1.225 & -1.056 & -0.169 & -1.004 & -0.841 & -0.163 \\
\hline K63 & 8.080 & 120.178 & 8.500 & 120.591 & -0.420 & -0.237 & -0.183 & -0.413 & -0.280 & -0.133 \\
\hline E64 & 9.686 & 115.181 & 9.465 & 114.976 & 0.221 & 0.121 & 0.100 & 0.205 & 0.078 & 0.127 \\
\hline S65 & - & - & 7.729 & 115.217 & - & - & - & - & - & - \\
\hline T66 & - & - & 8.872 & 117.747 & - & - & - & - & - & - \\
\hline L67 & - & - & 9.340 & 127.737 & - & - & - & - & - & - \\
\hline H68 & - & - & 9.329 & 120.108 & - & - & - & - & - & - \\
\hline L69 & - & - & 8.213 & 123.981 & - & - & - & - & - & - \\
\hline V70 & 8.881 & 126.443 & 9.184 & 126.626 & -0.303 & -0.179 & -0.124 & -0.183 & -0.113 & -0.070 \\
\hline L71 & 8.041 & 122.853 & 8.117 & 122.964 & -0.076 & 0.130 & -0.206 & -0.111 & 0.084 & -0.195 \\
\hline R72 & 8.487 & 123.616 & 8.610 & 123.734 & -0.123 & 0.039 & -0.162 & -0.118 & 0.048 & -0.166 \\
\hline L73 & 8.248 & 124.525 & 8.401 & 124.667 & -0.153 & 0.070 & -0.223 & -0.142 & 0.067 & -0.209 \\
\hline R74 & 8.369 & 122.011 & 8.480 & 122.133 & -0.111 & 0.052 & -0.163 & -0.122 & 0.051 & -0.173 \\
\hline G75 & 8.426 & 111.072 & 8.518 & 111.191 & -0.092 & 0.047 & -0.139 & -0.119 & 0.045 & -0.164 \\
\hline G76 & 7.883 & 115.149 & 7.989 & 115.258 & -0.106 & 0.050 & -0.156 & -0.109 & 0.048 & -0.157 \\
\hline
\end{tabular}


Tab. 7.3: 65 Pseudokontaktverschiebungen von T12C Tm [ppm]. Als diamagnetische Referenzprobe wurde T12C Lu verwendet. Gegeben sind die zugrunde liegenden chemischen Verschiebungen, sowie die experimentellen und berechneten PCSs und deren Differenz $\Delta$ für ${ }^{1} \mathrm{H}$ und ${ }^{15} \mathrm{~N}$. Die Daten wurden bei $298 \mathrm{~K}$ aufgenommen.

\begin{tabular}{|c|c|c|c|c|c|c|c|c|c|c|}
\hline \multirow[b]{2}{*}{$\mathrm{AS}$} & \multicolumn{2}{|c|}{ Thulium } & \multicolumn{2}{|c|}{ Lutetium } & \multicolumn{3}{|c|}{$\operatorname{PCS}{ }^{1} \mathrm{H}$} & \multicolumn{3}{|c|}{$\operatorname{PCS}{ }^{15} \mathrm{~N}$} \\
\hline & ${ }^{1} \mathrm{H}$ & ${ }^{15} \mathrm{~N}$ & ${ }^{1} \mathrm{H}$ & ${ }^{15} \mathrm{~N}$ & $\exp$ & ber & $\Delta$ & $\exp$ & ber & $\Delta$ \\
\hline $\mathrm{Q} 2$ & 8.987 & 123.488 & 8.951 & 123.475 & 0.036 & 0.063 & -0.027 & 0.013 & 0.056 & -0.043 \\
\hline I3 & 8.301 & 115.792 & 8.391 & 115.876 & -0.090 & -0.078 & -0.012 & -0.084 & -0.034 & -0.050 \\
\hline $\mathrm{F} 4$ & 8.578 & 117.883 & 8.793 & 118.262 & -0.215 & -0.125 & -0.090 & -0.379 & -0.306 & -0.073 \\
\hline $\mathrm{V} 5$ & 8.629 & 120.212 & 9.254 & 120.774 & -0.625 & -0.715 & 0.090 & -0.562 & -0.627 & 0.065 \\
\hline $\mathrm{K} 6$ & 8.620 & 128.677 & 9.113 & 129.453 & -0.493 & -0.695 & 0.202 & -0.776 & -0.907 & 0.131 \\
\hline $\mathrm{T} 7$ & 7.843 & 116.999 & 8.887 & 117.897 & -1.044 & -1.030 & -0.014 & -0.898 & -0.841 & -0.057 \\
\hline $\mathrm{L} 8$ & 8.569 & 120.369 & 9.065 & 120.995 & -0.496 & -0.297 & -0.199 & -0.626 & -0.381 & -0.245 \\
\hline $\mathrm{T} 9$ & 7.045 & 105.467 & 7.657 & 106.085 & -0.612 & -0.476 & -0.136 & -0.618 & -0.469 & -0.149 \\
\hline G10 & 6.886 & 109.036 & 7.989 & 110.048 & -1.103 & -0.844 & -0.259 & -1.012 & -0.893 & -0.119 \\
\hline K11 & 6.388 & 120.404 & 7.210 & 121.375 & -0.822 & -0.967 & 0.145 & -0.971 & -1.136 & 0.165 \\
\hline $\mathrm{C} 12$ & - & - & 8.726 & 123.497 & - & - & - & - & - & - \\
\hline $\mathrm{I} 13$ & 8.581 & 123.731 & 9.575 & 124.879 & -0.994 & -0.964 & -0.030 & -1.148 & -0.957 & -0.191 \\
\hline $\mathrm{T} 14$ & 8.085 & 119.876 & 8.696 & 120.484 & -0.611 & -0.609 & -0.002 & -0.608 & -0.626 & 0.018 \\
\hline $\mathrm{L} 15$ & 8.569 & 124.860 & 8.845 & 125.132 & -0.276 & -0.294 & 0.018 & -0.272 & -0.288 & 0.016 \\
\hline E16 & 8.093 & 122.676 & 8.148 & 122.747 & -0.055 & -0.096 & 0.041 & -0.071 & -0.089 & 0.018 \\
\hline V17 & 9.058 & 117.848 & 8.979 & 117.766 & 0.079 & 0.068 & 0.011 & 0.082 & 0.064 & 0.018 \\
\hline E18 & 8.866 & 119.628 & 8.701 & 119.472 & 0.165 & 0.164 & 0.001 & 0.156 & 0.167 & -0.011 \\
\hline $\mathrm{S} 20$ & 7.301 & 103.788 & 7.072 & 103.568 & 0.229 & 0.226 & 0.003 & 0.220 & 0.244 & -0.024 \\
\hline D21 & 8.344 & 124.345 & 8.111 & 124.124 & 0.233 & 0.230 & 0.003 & 0.221 & 0.217 & 0.004 \\
\hline $\mathrm{T} 22$ & 8.098 & 109.284 & 7.917 & 109.099 & 0.181 & 0.180 & 0.003 & 0.185 & 0.205 & -0.020 \\
\hline $\mathrm{I} 23$ & 8.826 & 121.686 & 8.559 & 121.431 & 0.267 & 0.272 & -0.005 & 0.255 & 0.261 & -0.006 \\
\hline N25 & 8.121 & 121.651 & 7.965 & 121.551 & 0.156 & 0.157 & -0.001 & 0.100 & 0.138 & -0.038 \\
\hline V26 & 8.319 & 122.496 & 8.148 & 122.367 & 0.171 & 0.167 & 0.004 & 0.129 & 0.138 & -0.009 \\
\hline $\mathrm{K} 27$ & 8.750 & 119.288 & 8.602 & 119.163 & 0.148 & 0.142 & 0.006 & 0.125 & 0.115 & 0.010 \\
\hline A 28 & 8.063 & 123.629 & 7.980 & 123.583 & 0.083 & 0.076 & 0.007 & 0.046 & 0.055 & -0.009 \\
\hline K29 & 7.933 & 120.388 & 7.883 & 120.383 & 0.050 & 0.030 & 0.020 & 0.005 & 0.008 & -0.003 \\
\hline I30 & 8.371 & 121.564 & 8.363 & 121.579 & 0.008 & -0.014 & 0.022 & -0.015 & -0.046 & 0.031 \\
\hline Q31 & 8.573 & 123.592 & 8.580 & 123.688 & -0.007 & -0.027 & 0.020 & -0.096 & -0.049 & -0.047 \\
\hline D32 & 7.989 & 119.734 & 8.009 & 119.792 & -0.020 & -0.053 & 0.033 & -0.058 & -0.066 & 0.008 \\
\hline K33 & 7.397 & 115.688 & 7.468 & 115.775 & -0.071 & -0.117 & 0.046 & -0.087 & -0.127 & 0.040 \\
\hline E34 & 8.691 & 114.559 & 8.801 & 114.662 & -0.110 & -0.147 & 0.037 & -0.103 & -0.166 & 0.063 \\
\hline G35 & 8.424 & 108.861 & 8.501 & 108.935 & -0.077 & -0.109 & 0.032 & -0.074 & -0.113 & 0.039 \\
\hline I36 & 6.127 & 120.384 & 6.198 & 120.455 & -0.071 & -0.088 & 0.017 & -0.071 & -0.075 & 0.004 \\
\hline D39 & 8.615 & 113.800 & 8.572 & 113.785 & 0.043 & 0.058 & -0.015 & 0.015 & 0.071 & -0.056 \\
\hline Q40 & 7.901 & 117.041 & 7.866 & 117.011 & 0.035 & 0.058 & -0.023 & 0.030 & 0.069 & -0.039 \\
\hline Q41 & 7.593 & 118.372 & 7.524 & 118.286 & 0.069 & 0.092 & -0.023 & 0.086 & 0.093 & -0.007 \\
\hline
\end{tabular}


Tab. 7.3: Fortsetzung.

\begin{tabular}{|c|c|c|c|c|c|c|c|c|c|c|}
\hline \multirow[b]{2}{*}{$\mathrm{AS}$} & \multicolumn{2}{|c|}{ Thulium } & \multicolumn{2}{|c|}{ Lutetium } & \multicolumn{3}{|c|}{$\operatorname{PCS}{ }^{1} \mathrm{H}$} & \multicolumn{3}{|c|}{$\operatorname{PCS}{ }^{15} \mathrm{~N}$} \\
\hline & ${ }^{1} \mathrm{H}$ & ${ }^{15} \mathrm{~N}$ & ${ }^{1} \mathrm{H}$ & ${ }^{15} \mathrm{~N}$ & $\exp$ & ber & $\Delta$ & $\exp$ & ber & $\Delta$ \\
\hline $\mathrm{R} 42$ & 8.713 & 123.502 & 8.539 & 123.329 & 0.174 & 0.179 & -0.005 & 0.173 & 0.200 & -0.027 \\
\hline L43 & 9.234 & 124.920 & 8.816 & 124.468 & 0.418 & 0.377 & 0.041 & 0.452 & 0.392 & 0.060 \\
\hline I44 & 10.166 & 123.682 & 9.169 & 122.725 & 0.997 & 0.761 & 0.236 & 0.957 & 0.812 & 0.145 \\
\hline F45 & 10.349 & 126.476 & 8.859 & 124.713 & 1.490 & 1.470 & 0.020 & 1.763 & 1.649 & 0.114 \\
\hline A 46 & - & - & 9.142 & 133.633 & - & - & - & - & - & - \\
\hline G47 & - & - & 8.295 & 106.775 & - & - & - & - & - & - \\
\hline K48 & 9.440 & 123.246 & 8.034 & 122.131 & 1.406 & 1.599 & -0.193 & 1.115 & 1.320 & -0.205 \\
\hline Q49 & 9.257 & 123.565 & 8.664 & 122.923 & 0.593 & 0.654 & -0.061 & 0.642 & 0.736 & -0.094 \\
\hline L50 & 9.266 & 126.595 & 8.631 & 125.972 & 0.635 & 0.639 & -0.004 & 0.623 & 0.613 & 0.010 \\
\hline E51 & 8.826 & 123.731 & 8.432 & 123.360 & 0.394 & 0.423 & -0.029 & 0.371 & 0.399 & -0.028 \\
\hline D52 & 8.440 & 120.895 & 8.211 & 120.650 & 0.229 & 0.242 & -0.013 & 0.245 & 0.254 & -0.009 \\
\hline R54 & 7.783 & 119.849 & 7.532 & 119.598 & 0.251 & 0.271 & -0.020 & 0.251 & 0.261 & -0.010 \\
\hline $\mathrm{T} 55$ & 9.179 & 109.229 & 8.870 & 108.963 & 0.309 & 0.325 & -0.016 & 0.266 & 0.324 & -0.058 \\
\hline L56 & 8.537 & 118.629 & 8.183 & 118.229 & 0.354 & 0.370 & -0.016 & 0.400 & 0.419 & -0.019 \\
\hline $\mathrm{S} 57$ & 8.943 & 114.059 & 8.535 & 113.605 & 0.408 & 0.414 & -0.006 & 0.454 & 0.470 & -0.016 \\
\hline D58 & 8.397 & 125.090 & 7.977 & 124.679 & 0.420 & 0.437 & -0.017 & 0.411 & 0.465 & -0.054 \\
\hline Y59 & 7.904 & 116.552 & 7.312 & 115.960 & 0.592 & 0.626 & -0.034 & 0.592 & 0.645 & -0.053 \\
\hline N60 & 8.896 & 117.020 & 8.189 & 116.145 & 0.707 & 0.698 & 0.009 & 0.875 & 0.813 & 0.062 \\
\hline I61 & 8.146 & 120.157 & 7.254 & 119.080 & 0.892 & 0.884 & 0.008 & 1.077 & 1.016 & 0.061 \\
\hline Q62 & 9.243 & 126.416 & 7.770 & 125.201 & 1.473 & 1.365 & 0.108 & 1.215 & 1.115 & 0.100 \\
\hline K63 & 9.168 & 121.296 & 8.500 & 120.591 & 0.668 & 0.628 & 0.040 & 0.705 & 0.664 & 0.041 \\
\hline E64 & 9.656 & 115.252 & 9.465 & 114.976 & 0.191 & 0.259 & -0.068 & 0.276 & 0.358 & -0.082 \\
\hline S65 & 8.397 & 115.897 & 7.729 & 115.217 & 0.668 & 0.686 & -0.018 & 0.680 & 0.779 & -0.099 \\
\hline T66 & - & - & 8.872 & 117.747 & - & - & - & - & - & - \\
\hline L67 & 9.476 & 128.116 & 9.340 & 127.737 & 0.136 & 0.198 & -0.062 & 0.379 & 0.603 & -0.224 \\
\hline H68 & - & - & 9.329 & 120.108 & - & - & - & - & - & - \\
\hline L69 & 8.242 & 124.313 & 8.213 & 123.981 & 0.029 & -0.047 & 0.076 & 0.332 & 0.123 & 0.209 \\
\hline V70 & 9.466 & 126.920 & 9.184 & 126.626 & 0.282 & 0.217 & 0.065 & 0.294 & 0.181 & 0.113 \\
\hline L71 & 8.093 & 123.030 & 8.117 & 122.964 & -0.024 & 0.082 & -0.106 & 0.066 & 0.088 & -0.022 \\
\hline R72 & 8.646 & 123.772 & 8.610 & 123.734 & 0.036 & 0.078 & -0.042 & 0.038 & 0.086 & -0.048 \\
\hline L73 & 8.422 & 124.689 & 8.401 & 124.667 & 0.021 & 0.074 & -0.053 & 0.022 & 0.064 & -0.042 \\
\hline R74 & 8.469 & 122.127 & 8.480 & 122.133 & -0.011 & 0.046 & -0.057 & -0.006 & 0.050 & -0.056 \\
\hline G75 & 8.512 & 111.191 & 8.518 & 111.191 & -0.006 & 0.043 & -0.049 & 0.000 & 0.045 & -0.045 \\
\hline G76 & 7.985 & 115.248 & 7.989 & 115.258 & -0.004 & 0.039 & -0.043 & -0.010 & 0.041 & -0.051 \\
\hline
\end{tabular}


Tab. 7.4: 54 Pseudokontaktverschiebungen von S57C Tb [ppm]. Als diamagnetische Referenzprobe wurde S57C Lu verwendet. Gegeben sind die zugrunde liegenden chemischen Verschiebungen, sowie die experimentellen und berechneten PCSs und deren Differenz $\Delta$ für ${ }^{1} \mathrm{H}$ und ${ }^{15} \mathrm{~N}$. Die Daten wurden bei $298 \mathrm{~K}$ aufgenommen.

\begin{tabular}{|c|c|c|c|c|c|c|c|c|c|c|}
\hline \multirow[b]{2}{*}{$\mathrm{AS}$} & \multicolumn{2}{|c|}{ Terbium } & \multicolumn{2}{|c|}{ Lutetium } & \multicolumn{3}{|c|}{$\operatorname{PCS}{ }^{1} \mathrm{H}$} & \multicolumn{3}{|c|}{$\operatorname{PCS}{ }^{15} \mathrm{~N}$} \\
\hline & ${ }^{1} \mathrm{H}$ & ${ }^{15} \mathrm{~N}$ & ${ }^{1} \mathrm{H}$ & ${ }^{15} \mathrm{~N}$ & $\exp$ & ber & $\Delta$ & $\exp$ & ber & $\Delta$ \\
\hline $\mathrm{Q} 2$ & - & - & 8.872 & 123.052 & - & - & - & - & - & - \\
\hline I3 & - & - & 8.206 & 115.198 & - & - & - & - & - & - \\
\hline $\mathrm{F} 4$ & 9.268 & 119.195 & 8.542 & 118.579 & 0.726 & 0.697 & 0.029 & 0.616 & 0.614 & 0.002 \\
\hline V5 & 9.540 & 121.565 & 9.189 & 121.155 & 0.351 & 0.346 & 0.005 & 0.410 & 0.403 & 0.007 \\
\hline K6 & 9.232 & 128.356 & 8.902 & 128.063 & 0.330 & 0.326 & 0.004 & 0.293 & 0.291 & 0.002 \\
\hline $\mathrm{T} 7$ & 8.807 & 115.656 & 8.653 & 115.460 & 0.154 & 0.163 & -0.009 & 0.196 & 0.181 & 0.015 \\
\hline L8 & 9.165 & 121.353 & 9.035 & 121.227 & 0.130 & 0.149 & -0.019 & 0.126 & 0.144 & -0.018 \\
\hline T9 & 7.641 & 105.982 & 7.552 & 105.878 & 0.089 & 0.109 & -0.020 & 0.104 & 0.102 & 0.002 \\
\hline G10 & 7.832 & 109.319 & 7.747 & 109.246 & 0.085 & 0.107 & -0.022 & 0.073 & 0.093 & -0.020 \\
\hline K11 & 7.250 & 121.975 & 7.179 & 121.891 & 0.071 & 0.097 & -0.026 & 0.084 & 0.089 & -0.005 \\
\hline $\mathrm{T} 12$ & 8.613 & 120.743 & 8.553 & 120.649 & 0.060 & 0.061 & -0.001 & 0.094 & 0.088 & 0.006 \\
\hline $\mathrm{I} 13$ & 9.657 & 127.747 & 9.483 & 127.650 & 0.174 & 0.191 & -0.017 & 0.097 & 0.148 & -0.051 \\
\hline $\mathrm{T} 14$ & 8.655 & 121.703 & 8.649 & 121.672 & 0.006 & 0.038 & -0.032 & 0.031 & 0.098 & -0.067 \\
\hline L15 & 9.133 & 125.956 & 8.696 & 125.510 & 0.437 & 0.400 & 0.037 & 0.446 & 0.312 & 0.134 \\
\hline E16 & - & - & 8.017 & 122.542 & - & - & - & - & - & - \\
\hline V17 & - & - & 8.848 & 117.494 & - & - & - & - & - & - \\
\hline E18 & - & - & 8.616 & 120.298 & - & - & - & - & - & - \\
\hline S20 & - & - & 7.006 & 103.385 & - & - & - & - & - & - \\
\hline D21 & - & - & 7.903 & 123.324 & - & - & - & - & - & - \\
\hline $\mathrm{T} 22$ & - & - & 7.661 & 108.069 & - & - & - & - & - & - \\
\hline $\mathrm{I} 23$ & 9.198 & 122.096 & 8.443 & 121.284 & 0.755 & 0.724 & 0.031 & 0.812 & 0.794 & 0.018 \\
\hline N25 & - & - & 7.843 & 121.392 & - & - & - & - & - & - \\
\hline $\mathrm{V} 26$ & - & - & 7.996 & 122.212 & - & - & - & - & - & - \\
\hline $\mathrm{K} 27$ & 9.053 & 119.302 & 8.448 & 118.807 & 0.605 & 0.561 & 0.044 & 0.495 & 0.540 & -0.045 \\
\hline $\mathrm{A} 28$ & 8.332 & 123.862 & 7.899 & 123.482 & 0.433 & 0.397 & 0.036 & 0.380 & 0.358 & 0.022 \\
\hline K29 & 8.266 & 120.618 & 7.777 & 120.248 & 0.489 & 0.454 & 0.035 & 0.370 & 0.376 & -0.006 \\
\hline I30 & 8.609 & 121.835 & 8.201 & 121.474 & 0.408 & 0.388 & 0.020 & 0.361 & 0.333 & 0.028 \\
\hline Q31 & 8.688 & 123.673 & 8.455 & 123.495 & 0.233 & 0.241 & -0.008 & 0.178 & 0.210 & -0.032 \\
\hline D32 & 8.052 & 119.817 & 7.922 & 119.736 & 0.130 & 0.167 & -0.037 & 0.081 & 0.136 & -0.055 \\
\hline K33 & 7.441 & 115.531 & 7.350 & 115.493 & 0.091 & 0.132 & -0.041 & 0.038 & 0.097 & -0.059 \\
\hline E34 & 8.731 & 114.422 & 8.641 & 114.355 & 0.090 & 0.120 & -0.030 & 0.067 & 0.095 & -0.028 \\
\hline G35 & 8.459 & 108.959 & 8.404 & 108.875 & 0.055 & 0.097 & -0.042 & 0.084 & 0.088 & -0.004 \\
\hline I36 & 6.169 & 120.399 & 6.068 & 120.303 & 0.101 & 0.120 & -0.019 & 0.096 & 0.111 & -0.015 \\
\hline D39 & 8.565 & 113.773 & 8.442 & 113.616 & 0.123 & 0.132 & -0.009 & 0.157 & 0.144 & 0.013 \\
\hline Q40 & 7.883 & 117.058 & 7.739 & 116.861 & 0.144 & 0.141 & 0.003 & 0.197 & 0.139 & 0.058 \\
\hline Q41 & 7.580 & 118.362 & 7.396 & 118.124 & 0.184 & 0.182 & 0.002 & 0.238 & 0.183 & 0.055 \\
\hline
\end{tabular}


Tab. 7.4: Fortsetzung.

\begin{tabular}{|c|c|c|c|c|c|c|c|c|c|c|}
\hline \multirow[b]{2}{*}{$\mathrm{AS}$} & \multicolumn{2}{|c|}{ Terbium } & \multicolumn{2}{|c|}{ Lutetium } & \multicolumn{3}{|c|}{$\mathrm{PCS}{ }^{1} \mathrm{H}$} & \multicolumn{3}{|c|}{ PCS ${ }^{15} \mathrm{~N}$} \\
\hline & ${ }^{1} \mathrm{H}$ & ${ }^{15} \mathrm{~N}$ & ${ }^{1} \mathrm{H}$ & ${ }^{15} \mathrm{~N}$ & $\exp$ & ber & $\Delta$ & $\exp$ & ber & $\Delta$ \\
\hline $\mathrm{R} 42$ & 8.646 & 123.533 & 8.409 & 123.217 & 0.237 & 0.238 & -0.001 & 0.316 & 0.245 & 0.071 \\
\hline $\mathrm{L} 43$ & 9.072 & 124.897 & 8.715 & 124.496 & 0.357 & 0.353 & 0.004 & 0.401 & 0.354 & 0.047 \\
\hline $\mathrm{I} 44$ & 9.449 & 122.742 & 9.047 & 122.344 & 0.402 & 0.410 & -0.008 & 0.398 & 0.415 & -0.017 \\
\hline F 45 & 9.194 & 125.401 & 8.748 & 124.895 & 0.446 & 0.464 & -0.018 & 0.506 & 0.485 & 0.021 \\
\hline A 46 & 9.317 & 133.291 & 8.901 & 132.870 & 0.416 & 0.465 & -0.049 & 0.421 & 0.460 & -0.039 \\
\hline G47 & 8.274 & 102.704 & 7.999 & 102.436 & 0.275 & 0.351 & -0.076 & 0.268 & 0.341 & -0.073 \\
\hline K48 & 8.203 & 122.325 & 7.909 & 122.058 & 0.294 & 0.381 & -0.087 & 0.267 & 0.346 & -0.079 \\
\hline Q49 & 8.784 & 123.349 & 8.546 & 123.058 & 0.238 & 0.298 & -0.060 & 0.291 & 0.328 & -0.037 \\
\hline L50 & 8.853 & 125.966 & 8.458 & 125.632 & 0.395 & 0.424 & -0.029 & 0.334 & 0.420 & -0.086 \\
\hline E51 & 8.706 & 123.519 & 8.342 & 123.136 & 0.364 & 0.424 & -0.060 & 0.383 & 0.401 & -0.018 \\
\hline D52 & 8.330 & 120.620 & 8.074 & 120.255 & 0.256 & 0.271 & -0.015 & 0.365 & 0.309 & 0.056 \\
\hline $\mathrm{R} 54$ & 7.694 & 119.556 & 7.346 & 119.270 & 0.348 & 0.371 & -0.023 & 0.286 & 0.341 & -0.055 \\
\hline $\mathrm{T} 55$ & - & - & 8.885 & 109.171 & - & - & - & - & - & - \\
\hline $\mathrm{L} 56$ & 10.126 & 119.349 & 8.093 & 117.596 & 2.033 & 1.976 & 0.057 & 1.753 & 1.798 & -0.045 \\
\hline C57 & - & - & 7.935 & 116.710 & - & - & - & - & - & - \\
\hline D58 & - & - & 7.838 & 122.117 & - & - & - & - & - & - \\
\hline Y59 & 8.248 & 116.265 & 7.089 & 115.262 & 1.159 & 1.195 & -0.036 & 1.003 & 1.028 & -0.025 \\
\hline N60 & 9.415 & 117.952 & 8.113 & 116.590 & 1.302 & 1.235 & 0.067 & 1.362 & 1.209 & 0.153 \\
\hline I61 & 9.021 & 120.505 & 7.308 & 118.889 & 1.713 & 1.749 & -0.036 & 1.616 & 1.657 & -0.041 \\
\hline Q62 & 8.922 & 126.552 & 7.406 & 124.638 & 1.516 & 1.520 & -0.004 & 1.914 & 1.847 & 0.067 \\
\hline K63 & - & - & 8.503 & 120.763 & - & - & - & - & - & - \\
\hline E64 & - & - & 9.203 & 114.330 & - & - & - & - & - & - \\
\hline $\mathrm{S} 65$ & - & - & 7.560 & 114.865 & - & - & - & - & - & - \\
\hline T66 & 9.179 & 118.011 & 8.621 & 117.415 & 0.558 & 0.624 & -0.066 & 0.596 & 0.643 & -0.047 \\
\hline $\mathrm{L} 67$ & 9.897 & 128.337 & 9.309 & 127.736 & 0.588 & 0.582 & 0.006 & 0.601 & 0.587 & 0.014 \\
\hline $\mathrm{H} 68$ & 9.555 & 120.036 & 9.122 & 119.647 & 0.433 & 0.458 & -0.025 & 0.389 & 0.430 & -0.041 \\
\hline L69 & 8.468 & 124.061 & 8.190 & 123.755 & 0.278 & 0.288 & -0.010 & 0.306 & 0.308 & -0.002 \\
\hline V70 & 9.331 & 126.674 & 9.085 & 126.492 & 0.246 & 0.256 & -0.010 & 0.182 & 0.237 & -0.055 \\
\hline L71 & 8.180 & 123.234 & 8.026 & 123.064 & 0.154 & 0.157 & -0.003 & 0.170 & 0.164 & 0.006 \\
\hline $\mathrm{R} 72$ & 8.643 & 123.873 & 8.503 & 123.679 & 0.140 & 0.136 & 0.004 & 0.194 & 0.131 & 0.063 \\
\hline $\mathrm{L} 73$ & 8.379 & 124.649 & 8.265 & 124.509 & 0.114 & 0.095 & 0.019 & 0.140 & 0.094 & 0.046 \\
\hline $\mathrm{R} 74$ & 8.443 & 122.073 & 8.349 & 121.956 & 0.094 & 0.075 & 0.019 & 0.117 & 0.073 & 0.044 \\
\hline G75 & 8.478 & 111.140 & 8.392 & 111.040 & 0.086 & 0.060 & 0.026 & 0.100 & 0.060 & 0.040 \\
\hline G76 & 7.926 & 115.160 & 7.858 & 115.077 & 0.068 & 0.053 & 0.015 & 0.083 & 0.053 & 0.030 \\
\hline
\end{tabular}


Tab. 7.5: 48 Pseudokontaktverschiebungen von S57C Tm [ppm]. Als diamagnetische Referenzprobe wurde S57C Lu verwendet. Gegeben sind die zugrunde liegenden chemischen Verschiebungen, sowie die experimentellen und berechneten PCSs und deren Differenz $\Delta$ für ${ }^{1} \mathrm{H}$ und ${ }^{15} \mathrm{~N}$. Die Daten wurden bei $298 \mathrm{~K}$ aufgenommen.

\begin{tabular}{|c|c|c|c|c|c|c|c|c|c|c|}
\hline \multirow[b]{2}{*}{ AS } & \multicolumn{2}{|c|}{ Thulium } & \multicolumn{2}{|c|}{ Lutetium } & \multicolumn{3}{|c|}{$\mathrm{PCS}{ }^{1} \mathrm{H}$} & \multicolumn{3}{|c|}{$\mathrm{PCS}{ }^{15} \mathrm{~N}$} \\
\hline & ${ }^{1} \mathrm{H}$ & ${ }^{15} \mathrm{~N}$ & ${ }^{1} \mathrm{H}$ & ${ }^{15} \mathrm{~N}$ & $\exp$ & ber & $\Delta$ & exp & ber & $\Delta$ \\
\hline $\mathrm{Q} 2$ & - & - & 8.872 & 123.052 & - & - & - & - & - & - \\
\hline I3 & - & - & 8.206 & 115.198 & - & - & - & - & - & - \\
\hline F4 & 8.133 & 118.137 & 8.542 & 118.579 & -0.409 & -0.397 & -0.012 & -0.442 & -0.391 & -0.051 \\
\hline $\mathrm{V} 5$ & 8.921 & 120.856 & 9.189 & 121.155 & -0.268 & -0.286 & 0.018 & -0.299 & -0.295 & -0.004 \\
\hline K6 & 8.710 & 127.868 & 8.902 & 128.063 & -0.192 & -0.198 & 0.006 & -0.195 & -0.189 & -0.006 \\
\hline $\mathrm{T} 7$ & 8.531 & 115.345 & 8.653 & 115.460 & -0.122 & -0.122 & 0.000 & -0.115 & -0.126 & 0.011 \\
\hline L8 & 8.944 & 121.135 & 9.035 & 121.227 & -0.091 & -0.091 & 0.000 & -0.092 & -0.089 & -0.003 \\
\hline T9 & 7.482 & 105.813 & 7.552 & 105.878 & -0.070 & -0.079 & 0.009 & -0.065 & -0.073 & 0.008 \\
\hline G10 & 7.674 & 109.194 & 7.747 & 109.246 & -0.073 & -0.079 & 0.006 & -0.052 & -0.074 & 0.022 \\
\hline K11 & 7.094 & 121.813 & 7.179 & 121.891 & -0.085 & -0.090 & 0.005 & -0.078 & -0.088 & 0.010 \\
\hline $\mathrm{T} 12$ & 8.460 & 120.554 & 8.553 & 120.649 & -0.093 & -0.108 & 0.015 & -0.095 & -0.118 & 0.023 \\
\hline $\mathrm{I} 13$ & 9.304 & 127.454 & 9.483 & 127.650 & -0.179 & -0.201 & 0.022 & -0.196 & -0.194 & -0.002 \\
\hline $\mathrm{T} 14$ & 8.443 & 121.409 & 8.649 & 121.672 & -0.206 & -0.262 & 0.056 & -0.263 & -0.283 & 0.020 \\
\hline L15 & - & - & 8.696 & 125.510 & - & - & - & - & - & - \\
\hline E16 & - & - & 8.017 & 122.542 & - & - & - & - & - & - \\
\hline V17 & - & - & 8.848 & 117.494 & - & - & - & - & - & - \\
\hline E18 & - & - & 8.616 & 120.298 & - & - & - & - & - & - \\
\hline $\mathrm{S} 20$ & - & - & 7.006 & 103.385 & - & - & - & - & - & - \\
\hline D21 & - & - & 7.903 & 123.324 & - & - & - & - & - & - \\
\hline $\mathrm{T} 22$ & - & - & 7.661 & 108.069 & - & - & - & - & - & - \\
\hline $\mathrm{I} 23$ & - & - & 8.443 & 121.284 & - & - & - & - & - & - \\
\hline N25 & - & - & 7.843 & 121.392 & - & - & - & - & - & - \\
\hline V26 & 8.006 & 122.153 & 7.996 & 122.212 & 0.010 & 0.008 & 0.002 & -0.059 & -0.090 & 0.031 \\
\hline $\mathrm{K} 27$ & 8.378 & 118.643 & 8.448 & 118.807 & -0.070 & -0.069 & -0.001 & -0.164 & -0.126 & -0.038 \\
\hline A28 & 7.884 & 123.409 & 7.899 & 123.482 & -0.015 & -0.034 & 0.019 & -0.073 & -0.071 & -0.002 \\
\hline K29 & 7.579 & 120.026 & 7.777 & 120.248 & -0.198 & -0.215 & 0.017 & -0.222 & -0.218 & -0.004 \\
\hline I30 & 7.956 & 121.234 & 8.201 & 121.474 & -0.245 & -0.250 & 0.005 & -0.240 & -0.257 & 0.017 \\
\hline Q31 & 8.301 & 123.307 & 8.455 & 123.495 & -0.154 & -0.147 & -0.007 & -0.188 & -0.150 & -0.038 \\
\hline D32 & 7.734 & 119.532 & 7.922 & 119.736 & -0.188 & -0.163 & -0.025 & -0.204 & -0.153 & -0.051 \\
\hline K33 & 7.119 & 115.262 & 7.350 & 115.493 & -0.231 & -0.212 & -0.019 & -0.231 & -0.197 & -0.034 \\
\hline E34 & 8.470 & 114.193 & 8.641 & 114.355 & -0.171 & -0.161 & -0.010 & -0.162 & -0.153 & -0.009 \\
\hline G35 & 8.272 & 108.751 & 8.404 & 108.875 & -0.132 & -0.117 & -0.015 & -0.124 & -0.105 & -0.019 \\
\hline I36 & 5.964 & 120.222 & 6.068 & 120.303 & -0.104 & -0.096 & -0.008 & -0.081 & -0.082 & 0.001 \\
\hline D39 & 8.440 & 113.575 & 8.442 & 113.616 & -0.002 & 0.012 & -0.014 & -0.041 & 0.011 & -0.052 \\
\hline Q40 & 7.713 & 116.824 & 7.739 & 116.861 & -0.026 & -0.015 & -0.011 & -0.037 & -0.013 & -0.024 \\
\hline Q41 & 7.353 & 118.084 & 7.396 & 118.124 & -0.043 & -0.029 & -0.014 & -0.040 & -0.039 & -0.001 \\
\hline
\end{tabular}


Tab. 7.5: Fortsetzung.

\begin{tabular}{|c|c|c|c|c|c|c|c|c|c|c|}
\hline \multirow[b]{2}{*}{ AS } & \multicolumn{2}{|c|}{ Thulium } & \multicolumn{2}{|c|}{ Lutetium } & \multicolumn{3}{|c|}{$\mathrm{PCS}{ }^{1} \mathrm{H}$} & \multicolumn{3}{|c|}{ PCS ${ }^{15} \mathrm{~N}$} \\
\hline & ${ }^{1} \mathrm{H}$ & ${ }^{15} \mathrm{~N}$ & ${ }^{1} \mathrm{H}$ & ${ }^{15} \mathrm{~N}$ & $\exp$ & ber & $\Delta$ & $\exp$ & ber & $\Delta$ \\
\hline $\mathrm{R} 42$ & 8.331 & 123.167 & 8.409 & 123.217 & -0.078 & -0.077 & -0.001 & -0.050 & -0.069 & 0.019 \\
\hline $\mathrm{L} 43$ & 8.623 & 124.432 & 8.715 & 124.496 & -0.092 & -0.093 & 0.001 & -0.064 & -0.111 & 0.047 \\
\hline $\mathrm{I} 44$ & 8.882 & 122.162 & 9.047 & 122.344 & -0.165 & -0.179 & 0.014 & -0.182 & -0.173 & -0.009 \\
\hline F45 & 8.574 & 124.729 & 8.748 & 124.895 & -0.174 & -0.212 & 0.038 & -0.166 & -0.229 & 0.063 \\
\hline A46 & 8.693 & 132.653 & 8.901 & 132.870 & -0.208 & -0.228 & 0.020 & -0.217 & -0.229 & 0.012 \\
\hline G47 & 7.866 & 102.306 & 7.999 & 102.436 & -0.133 & -0.165 & 0.032 & -0.130 & -0.161 & 0.031 \\
\hline K48 & 7.789 & 121.991 & 7.909 & 122.058 & -0.120 & -0.179 & 0.059 & -0.067 & -0.156 & 0.089 \\
\hline Q49 & 8.526 & 122.985 & 8.546 & 123.058 & -0.020 & -0.100 & 0.080 & -0.073 & -0.113 & 0.040 \\
\hline L50 & 8.366 & 125.530 & 8.458 & 125.632 & -0.092 & -0.124 & 0.032 & -0.102 & -0.111 & 0.009 \\
\hline E51 & - & - & 8.342 & 123.136 & - & - & - & - & - & - \\
\hline D52 & 8.162 & 120.374 & 8.074 & 120.255 & 0.088 & 0.138 & -0.050 & 0.119 & 0.161 & -0.042 \\
\hline $\mathrm{R} 54$ & - & - & 7.346 & 119.270 & - & - & - & - & - & - \\
\hline $\mathrm{T} 55$ & - & - & 8.885 & 109.171 & - & - & - & - & - & - \\
\hline $\mathrm{L} 56$ & - & - & 8.093 & 117.596 & - & - & - & - & - & - \\
\hline C57 & - & - & 7.935 & 116.710 & - & - & - & - & - & - \\
\hline D58 & - & - & 7.838 & 122.117 & - & - & - & - & - & - \\
\hline Y59 & 6.269 & 114.455 & 7.089 & 115.262 & -0.820 & -0.821 & 0.001 & -0.807 & -0.743 & -0.064 \\
\hline N60 & 6.854 & 115.398 & 8.113 & 116.590 & -1.259 & -1.273 & 0.014 & -1.192 & -1.172 & -0.020 \\
\hline $\mathrm{I} 61$ & - & - & 7.308 & 118.889 & - & - & - & - & - & - \\
\hline Q62 & - & - & 7.406 & 124.638 & - & - & - & - & - & - \\
\hline K63 & - & - & 8.503 & 120.763 & - & - & - & - & - & - \\
\hline E64 & - & - & 9.203 & 114.330 & - & - & - & - & - & - \\
\hline S65 & - & - & 7.560 & 114.865 & - & - & - & - & - & - \\
\hline T66 & 8.341 & 117.071 & 8.621 & 117.415 & -0.280 & -0.248 & -0.032 & -0.344 & -0.271 & -0.073 \\
\hline $\mathrm{L} 67$ & 8.990 & 127.401 & 9.309 & 127.736 & -0.319 & -0.324 & 0.005 & -0.335 & -0.315 & -0.020 \\
\hline H68 & 8.914 & 119.395 & 9.122 & 119.647 & -0.208 & -0.230 & 0.022 & -0.252 & -0.221 & -0.031 \\
\hline L69 & 8.043 & 123.634 & 8.190 & 123.755 & -0.147 & -0.155 & 0.008 & -0.121 & -0.159 & 0.038 \\
\hline V70 & 8.980 & 126.362 & 9.085 & 126.492 & -0.105 & -0.107 & 0.002 & -0.130 & -0.104 & -0.026 \\
\hline $\mathrm{L} 71$ & 7.950 & 122.999 & 8.026 & 123.064 & -0.076 & -0.068 & -0.008 & -0.065 & -0.068 & 0.003 \\
\hline $\mathrm{R} 72$ & 8.447 & 123.607 & 8.503 & 123.679 & -0.056 & -0.041 & -0.015 & -0.072 & -0.041 & -0.031 \\
\hline L73 & 8.229 & 124.454 & 8.265 & 124.509 & -0.036 & -0.026 & -0.010 & -0.055 & -0.025 & -0.030 \\
\hline $\mathrm{R} 74$ & 8.307 & 121.915 & 8.349 & 121.956 & -0.042 & -0.014 & -0.028 & -0.041 & -0.013 & -0.028 \\
\hline G75 & 8.367 & 111.011 & 8.392 & 111.040 & -0.025 & -0.008 & -0.017 & -0.029 & -0.008 & -0.021 \\
\hline G76 & 7.844 & 115.056 & 7.858 & 115.077 & -0.014 & -0.006 & -0.008 & -0.021 & -0.006 & -0.015 \\
\hline
\end{tabular}


Tab. 7.6: Chemische Verschiebungen in ${ }^{1} \mathrm{H}$ und ${ }^{15} \mathrm{~N}$ der Hauptpeaks und der 2. und 3. anisotropen Signalsätze, sowie der isotropen Signale von zehn ausgewählten Aminosäureresten bei verschiedenen Temperaturen [ppm].

\begin{tabular}{|c|c|c|c|c|c|c|c|c|c|}
\hline \multirow[b]{2}{*}{$\mathrm{T}[\mathrm{K}]$} & \multirow[b]{2}{*}{$\mathrm{AS}$} & \multicolumn{2}{|c|}{ Hauptpeak } & \multicolumn{2}{|c|}{ isotrop } & \multicolumn{2}{|c|}{ anisotrop 2} & \multicolumn{2}{|c|}{ anisotrop 3} \\
\hline & & ${ }^{1} \mathrm{H}$ & ${ }^{15} \mathrm{~N}$ & ${ }^{1} \mathrm{H}$ & ${ }^{15} \mathrm{~N}$ & ${ }^{1} \mathrm{H}$ & ${ }^{15} \mathrm{~N}$ & ${ }^{1} \mathrm{H}$ & ${ }^{15} \mathrm{~N}$ \\
\hline \multirow[t]{10}{*}{288} & E16 & 8.027 & 122.667 & 8.130 & 122.729 & 7.861 & 122.531 & 7.914 & 122.557 \\
\hline & $\mathrm{I} 23$ & 8.798 & 121.687 & 8.492 & 121.445 & 8.589 & 121.480 & 8.684 & 121.573 \\
\hline & K33 & 7.291 & 115.727 & 7.360 & 115.808 & 7.180 & 115.700 & 7.221 & 115.692 \\
\hline & D52 & 8.439 & 121.012 & 8.164 & 120.732 & 8.236 & 120.809 & 8.335 & 120.927 \\
\hline & $\mathrm{R} 54$ & 7.751 & 120.039 & 7.450 & 119.722 & 7.576 & 119.908 & 7.659 & 119.959 \\
\hline & $\mathrm{T} 55$ & 9.146 & 109.250 & 8.783 & 108.925 & 8.968 & 109.066 & 9.038 & 109.122 \\
\hline & $\mathrm{L} 56$ & 8.513 & 118.649 & 8.288 & 118.413 & 8.349 & 118.461 & 8.403 & 118.544 \\
\hline & D58 & 8.394 & 125.231 & 7.904 & 124.775 & 8.200 & 125.060 & 8.264 & 125.129 \\
\hline & Y59 & 7.915 & 116.735 & 7.212 & 116.031 & 7.656 & 116.475 & 7.752 & 116.587 \\
\hline & N60 & 8.930 & 117.223 & 8.109 & 116.212 & 8.692 & 116.924 & 8.773 & 117.063 \\
\hline \multirow[t]{10}{*}{298} & E16 & 8.093 & 122.676 & 8.144 & 122.737 & 7.950 & 122.555 & 7.997 & 122.581 \\
\hline & $\mathrm{I} 23$ & 8.826 & 121.686 & 8.560 & 121.427 & 8.643 & 121.512 & 8.712 & 121.580 \\
\hline & K33 & 7.397 & 115.688 & 7.467 & 115.758 & 7.298 & 115.683 & 7.339 & 115.678 \\
\hline & D52 & 8.440 & 120.895 & 8.208 & 120.645 & 8.259 & 120.731 & 8.350 & 120.817 \\
\hline & $\mathrm{R} 54$ & 7.783 & 119.849 & 7.526 & 119.577 & 7.633 & 119.742 & 7.697 & 119.778 \\
\hline & $\mathrm{T} 55$ & 9.179 & 109.229 & 8.867 & 108.962 & 9.020 & 109.067 & 9.075 & 109.124 \\
\hline & $\mathrm{L} 56$ & 8.537 & 118.629 & 8.183 & 118.224 & 8.337 & 118.425 & 8.386 & 118.460 \\
\hline & D58 & 8.397 & 125.090 & 7.978 & 124.696 & 8.222 & 124.929 & 8.273 & 124.977 \\
\hline & Y59 & 7.904 & 116.552 & 7.304 & 115.946 & 7.665 & 116.322 & 7.749 & 116.402 \\
\hline & N60 & 8.896 & 117.020 & 8.191 & 116.160 & 8.673 & 116.747 & 8.735 & 116.836 \\
\hline \multirow[t]{10}{*}{308} & E16 & 8.154 & 122.676 & 8.214 & 122.752 & 8.033 & 122.565 & 8.069 & 122.589 \\
\hline & $\mathrm{I} 23$ & 8.857 & 121.682 & 8.640 & 121.485 & 8.700 & 121.533 & 8.752 & 121.589 \\
\hline & K33 & 7.503 & 115.647 & 7.564 & 115.681 & 7.455 & 115.643 & 7.470 & 115.644 \\
\hline & D52 & 8.444 & 120.776 & 8.252 & 120.566 & 8.287 & 120.629 & 8.362 & 120.694 \\
\hline & $\mathrm{R} 54$ & 7.819 & 119.656 & 7.609 & 119.445 & 7.690 & 119.556 & 7.736 & 119.585 \\
\hline & $\mathrm{T} 55$ & 9.212 & 109.209 & 8.954 & 108.986 & 9.076 & 109.077 & 9.117 & 109.103 \\
\hline & $\mathrm{L} 56$ & 8.567 & 118.617 & 8.265 & 118.286 & 8.391 & 118.436 & 8.425 & 118.460 \\
\hline & D58 & 8.404 & 124.938 & 8.052 & 124.611 & 8.248 & 124.792 & 8.284 & 124.824 \\
\hline & Y59 & 7.900 & 116.373 & 7.393 & 115.860 & 7.686 & 116.165 & 7.746 & 116.227 \\
\hline & N60 & 8.868 & 116.817 & 8.269 & 116.101 & 8.665 & 116.562 & 8.711 & 116.634 \\
\hline \multirow[t]{6}{*}{315} & E16 & 8.190 & 122.671 & 8.256 & 122.752 & 8.075 & 122.563 & 8.113 & 122.599 \\
\hline & $\mathrm{I} 23$ & 8.878 & 121.680 & 8.691 & 121.506 & 8.737 & 121.542 & 8.778 & 121.593 \\
\hline & K33 & 7.574 & 115.618 & 7.635 & 115.695 & 7.459 & 115.604 & 7.536 & 115.624 \\
\hline & D52 & 8.448 & 120.696 & 8.282 & 120.517 & 8.306 & 120.562 & 8.367 & 120.638 \\
\hline & $\mathrm{R} 54$ & 7.844 & 119.525 & 7.662 & 119.348 & 7.728 & 119.444 & 7.764 & 119.460 \\
\hline & T55 & 9.233 & 109.196 & 9.008 & 108.998 & 9.110 & 109.078 & 9.147 & 109.106 \\
\hline
\end{tabular}


Tab. 7.6: Fortsetzung.

\begin{tabular}{cccccccccc}
\hline & \multicolumn{4}{c}{ Hauptpeak } & \multicolumn{2}{c}{ isotrop } & \multicolumn{2}{c}{ anisotrop 2} & \multicolumn{2}{c}{ anisotrop 3 } \\
$\mathrm{T}[\mathrm{K}]$ & $\mathrm{AS}$ & ${ }^{1} \mathrm{H}$ & ${ }^{15} \mathrm{~N}$ & ${ }^{1} \mathrm{H}$ & ${ }^{15} \mathrm{~N}$ & ${ }^{1} \mathrm{H}$ & ${ }^{15} \mathrm{~N}$ & ${ }^{1} \mathrm{H}$ & ${ }^{15} \mathrm{~N}$ \\
\hline & $\mathrm{L} 56$ & 8.588 & 118.614 & 8.325 & 118.315 & 8.430 & 118.461 & 8.459 & 118.474 \\
& $\mathrm{D} 58$ & 8.408 & 124.830 & 8.098 & 124.543 & 8.266 & 124.702 & 8.297 & 124.736 \\
& $\mathrm{Y} 59$ & 7.899 & 116.255 & 7.453 & 115.800 & 7.704 & 116.070 & 7.751 & 116.117 \\
& $\mathrm{~N} 60$ & 8.852 & 116.680 & 8.322 & 116.028 & 8.663 & 116.442 & 8.702 & 116.501 \\
\hline
\end{tabular}


Tab. 7.7: Datenhöhen [ ] (Sparky) der Hauptpeaks und der 2. und 3. anisotropen Signalsätze, sowie der isotropen Signale von zehn ausgewählten Aminosäureresten bei verschiedenen Temperaturen und die resultierenden prozentualen Verteilungen. Die chemischen Verschiebungen der Reste sind in Tabelle 7.6 angegeben.

\begin{tabular}{|c|c|c|c|c|c|c|c|c|c|}
\hline \multirow[b]{2}{*}{$\mathrm{T}[\mathrm{K}]$} & \multirow[b]{2}{*}{ AS } & \multicolumn{4}{|c|}{ Datenhöhe [ ] } & \multicolumn{4}{|c|}{ Verteilung [\%] } \\
\hline & & Hauptpeak & isotrop & anisotrop 2 & anisotrop 3 & Hauptpeak & isotrop & anisotrop 2 & anisotrop 3 \\
\hline \multirow[t]{11}{*}{288} & E16 & 11970307 & 99826 & 123050 & 221937 & 96.42 & 0.80 & 0.99 & 1.79 \\
\hline & $\mathrm{I} 23$ & 5781384 & 174003 & 106341 & 131833 & 93.35 & 2.81 & 1.72 & 2.13 \\
\hline & K33 & 12750358 & 339880 & 112913 & 314216 & 94.33 & 2.51 & 0.84 & 2.32 \\
\hline & D52 & 6875856 & 166036 & 145524 & 135447 & 93.90 & 2.27 & 1.99 & 1.85 \\
\hline & $\mathrm{R} 54$ & 11620404 & 164739 & 169097 & 235163 & 95.33 & 1.35 & 1.39 & 1.93 \\
\hline & $\mathrm{T} 55$ & 6629228 & 228928 & 188035 & 161583 & 91.97 & 3.18 & 2.61 & 2.24 \\
\hline & $\mathrm{L} 56$ & 8830404 & 206205 & 207730 & 109437 & 94.40 & 2.20 & 2.22 & 1.17 \\
\hline & D58 & 8777918 & 306550 & 190810 & 207023 & 92.57 & 3.23 & 2.01 & 2.18 \\
\hline & Y59 & 6629605 & 304799 & 147333 & 157086 & 91.58 & 4.21 & 2.04 & 2.17 \\
\hline & N60 & 7899876 & 187824 & 169242 & 145894 & 94.01 & 2.24 & 2.01 & 1.74 \\
\hline & \multicolumn{5}{|c|}{ Durchschnitt: } & 93.79 & 2.48 & 1.78 & 1.95 \\
\hline \multirow[t]{11}{*}{298} & E16 & 243704784 & 6890782 & 2511184 & 4618226 & 94.56 & 2.67 & 0.97 & 1.79 \\
\hline & $\mathrm{I} 23$ & 171244848 & 3813710 & 3333864 & 3161000 & 94.32 & 2.10 & 1.84 & 1.74 \\
\hline & K33 & 269823584 & 5618110 & 907322 & 5776001 & 95.64 & 1.99 & 0.32 & 2.05 \\
\hline & $\mathrm{D} 52$ & 169826576 & 4736590 & 4278376 & 3804888 & 92.98 & 2.59 & 2.34 & 2.08 \\
\hline & $\mathrm{R} 54$ & 263979776 & 3457340 & 5394668 & 4652464 & 95.13 & 1.25 & 1.94 & 1.68 \\
\hline & $\mathrm{T} 55$ & 147937984 & 4333326 & 3538591 & 3414356 & 92.91 & 2.72 & 2.22 & 2.14 \\
\hline & $\mathrm{L} 56$ & 211467616 & 3463481 & 5534548 & 4869477 & 93.85 & 1.54 & 2.46 & 2.16 \\
\hline & D58 & 198895536 & 6522027 & 4758978 & 4411932 & 92.69 & 3.04 & 2.22 & 2.06 \\
\hline & Y59 & 160950960 & 5863800 & 4212980 & 3551424 & 92.19 & 3.36 & 2.41 & 2.03 \\
\hline & N60 & 212528144 & 5038628 & 4544283 & 3871153 & 94.05 & 2.23 & 2.01 & 1.71 \\
\hline & \multicolumn{5}{|c|}{ Durchschnitt: } & 93.90 & 2.31 & 1.84 & 1.95 \\
\hline \multirow[t]{11}{*}{308} & E16 & 17655216 & 460713 & 159082 & 254867 & 95.28 & 2.49 & 0.86 & 1.38 \\
\hline & $\mathrm{I} 23$ & 15672879 & 327171 & 348065 & 283594 & 94.23 & 1.97 & 2.09 & 1.71 \\
\hline & K33 & 19884292 & 280651 & 345243 & 112047 & 96.42 & 1.36 & 1.67 & 0.54 \\
\hline & D52 & 13642497 & 325100 & 354553 & 308213 & 93.25 & 2.22 & 2.42 & 2.11 \\
\hline & $\mathrm{R} 54$ & 20457212 & 313750 & 427604 & 405277 & 94.69 & 1.45 & 1.98 & 1.88 \\
\hline & T55 & 10671648 & 356403 & 255612 & 252073 & 92.51 & 3.09 & 2.22 & 2.19 \\
\hline & L56 & 17483552 & 312188 & 422988 & 338643 & 94.21 & 1.68 & 2.28 & 1.82 \\
\hline & D58 & 15105381 & 449648 & 369000 & 235361 & 93.48 & 2.78 & 2.28 & 1.46 \\
\hline & Y59 & 13012360 & 418051 & 357033 & 256372 & 92.66 & 2.98 & 2.54 & 1.83 \\
\hline & N60 & 18227082 & 351899 & 425796 & 373197 & 94.06 & 1.82 & 2.20 & 1.93 \\
\hline & \multicolumn{5}{|c|}{ Durchschnitt: } & 94.19 & 2.11 & 2.01 & 1.69 \\
\hline
\end{tabular}


Tab. 7.7: Fortsetzung.

\begin{tabular}{|c|c|c|c|c|c|c|c|c|c|}
\hline \multirow[b]{2}{*}{$\mathrm{T}[\mathrm{K}]$} & \multirow[b]{2}{*}{ AS } & \multicolumn{4}{|c|}{ Datenhöhe [ ] } & \multicolumn{4}{|c|}{ Verteilung [\%] } \\
\hline & & Hauptpeak & isotrop & anisotrop 2 & anisotrop 3 & Hauptpeak & isotrop & anisotrop 2 & anisotrop 3 \\
\hline \multirow[t]{11}{*}{315} & E16 & 24269074 & 546393 & 258939 & 417786 & 95.20 & 2.14 & 1.02 & 1.64 \\
\hline & $\mathrm{I} 23$ & 23671842 & 624612 & 515546 & 497488 & 93.53 & 2.47 & 2.04 & 1.97 \\
\hline & K33 & 27346108 & 469525 & 204966 & 751034 & 95.05 & 1.63 & 0.71 & 2.61 \\
\hline & D52 & 19529354 & 524935 & 517418 & 421367 & 93.03 & 2.50 & 2.46 & 2.01 \\
\hline & $\mathrm{R} 54$ & 28507434 & 463230 & 677240 & 619030 & 94.19 & 1.53 & 2.24 & 2.05 \\
\hline & T55 & 14183552 & 458137 & 334234 & 318923 & 92.73 & 3.00 & 2.19 & 2.09 \\
\hline & L56 & 25314620 & 446752 & 670003 & 537650 & 93.87 & 1.66 & 2.48 & 1.99 \\
\hline & D58 & 20375628 & 583321 & 515003 & 472987 & 92.84 & 2.66 & 2.35 & 2.16 \\
\hline & Y59 & 18592062 & 575985 & 490733 & 382663 & 92.77 & 2.87 & 2.45 & 1.91 \\
\hline & N60 & 26231724 & 544060 & 579627 & 537868 & 94.04 & 1.95 & 2.08 & 1.93 \\
\hline & \multicolumn{5}{|c|}{ Durchschnitt: } & 93.87 & 2.15 & 1.97 & 2.01 \\
\hline
\end{tabular}


Tab. 7.8: T12C Lu (diamagnetische Referenz). Chemische Verschiebungen $\left({ }^{1} \mathrm{H}\right.$ und $\left.{ }^{15} \mathrm{~N}\right)$ der Amidsignale des Proteinrückgrats bei unterschiedlichen Temperaturen $[\mathrm{ppm}]$.

\begin{tabular}{|c|c|c|c|c|c|c|c|c|c|c|}
\hline \multirow[b]{2}{*}{$\mathrm{AS}$} & \multicolumn{2}{|c|}{$278 \mathrm{~K}$} & \multicolumn{2}{|c|}{$288 \mathrm{~K}$} & \multicolumn{2}{|c|}{$298 \mathrm{~K}$} & \multicolumn{2}{|c|}{$308 \mathrm{~K}$} & \multicolumn{2}{|c|}{$315 \mathrm{~K}$} \\
\hline & ${ }^{1} \mathrm{H}$ & ${ }^{15} \mathrm{~N}$ & ${ }^{1} \mathrm{H}$ & ${ }^{15} \mathrm{~N}$ & ${ }^{1} \mathrm{H}$ & ${ }^{15} \mathrm{~N}$ & ${ }^{1} \mathrm{H}$ & ${ }^{15} \mathrm{~N}$ & ${ }^{1} \mathrm{H}$ & ${ }^{15} \mathrm{~N}$ \\
\hline $\mathrm{Q} 2$ & 8.848 & 123.3 & 8.905 & 123 & 8.951 & 12 & 8.991 & 2 & 4 & 38 \\
\hline I3 & 8.168 & 115.633 & 8.284 & 115.750 & 8.391 & 115.876 & 8.494 & 116.006 & 8.561 & 116.095 \\
\hline $\mathrm{F} 4$ & 8.646 & 118.006 & 8.722 & 118.137 & 8.793 & 118.262 & 8.862 & 118.386 & 8.907 & 118.470 \\
\hline V5 & 9.081 & 120.673 & 9.171 & 120.725 & 9.254 & 120.774 & 9.336 & 120.821 & 9.390 & 120.858 \\
\hline K6 & 8.926 & 129.493 & 9.025 & 129.481 & 9.113 & 129.453 & 9.197 & 129.402 & 9.251 & 129.360 \\
\hline $\mathrm{T} 7$ & 8.780 & 118.317 & 8.835 & 118.115 & 8.887 & 117.897 & 8.941 & 117.657 & 8.977 & 117.488 \\
\hline L8 & 9.075 & 121.388 & 9.069 & 121.178 & 9.065 & 120.995 & 9.062 & 120.832 & 9.059 & 120.738 \\
\hline T9 & 7.487 & 106.165 & 7.575 & 106.125 & 7.657 & 106.085 & 7.737 & 106.039 & 7.799 & 106.020 \\
\hline G10 & 7.839 & 110.283 & 7.916 & 110.166 & 7.989 & 110.048 & 8.061 & 109.916 & 8.109 & 109.825 \\
\hline K11 & 7.028 & 121.771 & 7.121 & 121.571 & 7.210 & 121.375 & 7.298 & 121.175 & 7.357 & 121.039 \\
\hline $\mathrm{C} 12$ & 8.615 & 123.824 & 8.673 & 123.654 & 8.726 & 123.497 & 8.777 & 123.340 & 8.810 & 123.239 \\
\hline $\mathrm{I} 13$ & 9.448 & 125.332 & 9.514 & 125.102 & 9.575 & 124.879 & 9.633 & 124.673 & 9.672 & 124.553 \\
\hline $\mathrm{T} 14$ & 8.627 & 120.671 & 8.665 & 120.592 & 8.696 & 120.484 & 8.724 & 120.356 & 8.742 & 120.265 \\
\hline $\mathrm{L} 15$ & 8.687 & 125.051 & 8.770 & 125.105 & 8.845 & 125.132 & 8.917 & 125.131 & 8.962 & 125.123 \\
\hline E16 & 7.981 & 122.697 & 8.071 & 122.728 & 8.148 & 122.747 & 8.218 & 122.754 & 8.260 & 122.753 \\
\hline V17 & 8.826 & 117.691 & 8.907 & 117.736 & 8.979 & 117.766 & 9.046 & 117.778 & 9.087 & 117.778 \\
\hline E18 & 8.448 & 119.056 & 8.581 & 119.271 & 8.701 & 119.472 & 8.815 & 119.663 & 8.888 & 119.787 \\
\hline $\mathrm{S} 20$ & 6.899 & 103.334 & 6.988 & 103.449 & 7.072 & 103.568 & 7.157 & 103.686 & 7.213 & 103.769 \\
\hline $\mathrm{D} 21$ & 7.961 & 124.349 & 8.039 & 124.237 & 8.111 & 124.124 & 8.181 & 124.003 & 8.227 & 123.919 \\
\hline $\mathrm{T} 22$ & 7.804 & 109.273 & 7.861 & 109.183 & 7.917 & 109.099 & 7.974 & 109.011 & 8.013 & 108.953 \\
\hline $\mathrm{I} 23$ & 8.392 & 121.319 & 8.478 & 121.379 & 8.559 & 121.431 & 8.637 & 121.473 & 8.689 & 121.498 \\
\hline $\mathrm{N} 25$ & 7.810 & 121.712 & 7.890 & 121.641 & 7.965 & 121.551 & 8.038 & 121.439 & 8.086 & 121.356 \\
\hline V26 & 7.995 & 122.509 & 8.074 & 122.440 & 8.148 & 122.367 & 8.220 & 122.283 & 8.267 & 122.222 \\
\hline $\mathrm{K} 27$ & 8.440 & 119.304 & 8.524 & 119 & 8.602 & 119.163 & 8.677 & 119.083 & 8.727 & 119.027 \\
\hline A 28 & 7.779 & 123.745 & 7.882 & 123.665 & 7.980 & 123.583 & 8.074 & 123.488 & 8.137 & 123.420 \\
\hline K29 & 7.699 & 120.528 & 7.793 & 120.460 & 7.883 & 120.383 & 7.972 & 120.292 & 8.031 & 120.223 \\
\hline I30 & 8.220 & 121.860 & 8.294 & 121.715 & 8.363 & 121.579 & 8.432 & 121.442 & 8.478 & 121.349 \\
\hline Q31 & 8.395 & 123.715 & 8.492 & 123.709 & 8.580 & 123.688 & 8.664 & 123.655 & 8.721 & 123.630 \\
\hline D32 & 7.889 & 119.891 & 7.950 & 119.840 & 8.009 & 119.792 & 8.072 & 119.741 & 8.114 & 119.706 \\
\hline K33 & 7.255 & 115.860 & 7.364 & 115.818 & 7.468 & 115.775 & 7.570 & 115.726 & 7.639 & 115.691 \\
\hline E34 & 8.633 & 114.595 & 8.722 & 114.623 & 8.801 & 114.662 & 8.874 & 114.699 & 8.921 & 114.726 \\
\hline G35 & 8.351 & 109.114 & 8.429 & 109.026 & 8.501 & 108.935 & 8.572 & 108.833 & 8.619 & 108.762 \\
\hline I36 & 5.988 & 120.768 & 6.096 & 120.608 & 6.198 & 120.455 & 6.300 & 120.303 & 6.369 & 120.199 \\
\hline D39 & 8.441 & 114.061 & 8.507 & 113.909 & 8.572 & 113.785 & 8.639 & 113.667 & 8.686 & 113.597 \\
\hline Q40 & 7.708 & 117.026 & 7.790 & 117.030 & 7.866 & 117.011 & 7.942 & 116.967 & 7.993 & 116.929 \\
\hline Q41 & 7.405 & 118.651 & 7.465 & 118.445 & 7.524 & 118.286 & 7.586 & 118.152 & 7.628 & 118.073 \\
\hline $\mathrm{R} 42$ & 8.303 & 123.624 & 8.431 & 123.457 & 8.539 & 123.329 & 8.633 & 123.220 & 8.689 & 123.152 \\
\hline $\mathrm{L} 43$ & 8.690 & 124.629 & 8.759 & 124.515 & 8.816 & 124.468 & 8.874 & 124.437 & 8.911 & 124.431 \\
\hline $\mathrm{I} 44$ & 8.984 & 122.331 & 9.081 & 122.567 & 9.169 & 122.725 & 9.254 & 122.823 & 9.308 & 122.864 \\
\hline
\end{tabular}


Tab. 7.8: Fortsetzung.

\begin{tabular}{|c|c|c|c|c|c|c|c|c|c|c|}
\hline \multirow[b]{2}{*}{$\mathrm{AS}$} & \multicolumn{2}{|c|}{$278 \mathrm{~K}$} & \multicolumn{2}{|c|}{$288 \mathrm{~K}$} & \multicolumn{2}{|c|}{$298 \mathrm{~K}$} & \multicolumn{2}{|c|}{$308 \mathrm{~K}$} & \multicolumn{2}{|c|}{$315 \mathrm{~K}$} \\
\hline & ${ }^{1} \mathrm{H}$ & ${ }^{15} \mathrm{~N}$ & ${ }^{1} \mathrm{H}$ & ${ }^{15} \mathrm{~N}$ & ${ }^{1} \mathrm{H}$ & ${ }^{15} \mathrm{~N}$ & ${ }^{1} \mathrm{H}$ & ${ }^{15} \mathrm{~N}$ & ${ }^{1} \mathrm{H}$ & ${ }^{15} \mathrm{~N}$ \\
\hline $\mathrm{F} 45$ & 8.669 & 124.347 & 8.764 & 124.540 & 8.859 & 124.713 & 8.946 & 124.842 & 9.002 & 124.917 \\
\hline A 46 & 9.010 & 134.126 & 9.082 & 133.883 & 9.142 & 133.633 & 9.195 & 133.363 & 9.228 & 133.173 \\
\hline G47 & 8.234 & 106.910 & 8.267 & 106.848 & 8.295 & 106.775 & 8.319 & 106.686 & 8.333 & 106.621 \\
\hline K48 & 7.828 & 122.325 & 7.934 & 122.234 & 8.034 & 122.131 & 8.132 & 122.012 & 8.197 & 121.925 \\
\hline Q49 & 8.564 & 123.006 & 8.618 & 122.976 & 8.664 & 122.923 & 8.706 & 122.848 & 8.732 & 122.791 \\
\hline L50 & 8.467 & 125.981 & 8.556 & 125.988 & 8.631 & 125.972 & 8.699 & 125.934 & 8.741 & 125.899 \\
\hline E51 & 8.330 & 123.378 & 8.384 & 123.370 & 8.432 & 123.360 & 8.478 & 123.343 & 8.507 & 123.329 \\
\hline D52 & 8.117 & 120.786 & 8.166 & 120.720 & 8.211 & 120.650 & 8.254 & 120.573 & 8.284 & 120.517 \\
\hline $\mathrm{R} 54$ & 7.370 & 119.883 & 7.453 & 119.741 & 7.532 & 119.598 & 7.611 & 119.446 & 7.664 & 119.338 \\
\hline T55 & 8.682 & 108.887 & 8.781 & 108.929 & 8.870 & 108.963 & 8.956 & 108.988 & 9.011 & 109.004 \\
\hline L56 & 7.994 & 118.117 & 8.091 & 118.174 & 8.183 & 118.229 & 8.273 & 118.284 & 8.334 & 118.323 \\
\hline $\mathrm{S} 57$ & 8.422 & 113.730 & 8.480 & 113.670 & 8.535 & 113.605 & 8.588 & 113.530 & 8.623 & 113.476 \\
\hline D58 & 7.816 & 124.790 & 7.900 & 124.744 & 7.977 & 124.679 & 8.051 & 124.589 & 8.100 & 124.523 \\
\hline Y59 & 7.125 & 116.118 & 7.221 & 116.039 & 7.312 & 115.960 & 7.402 & 115.872 & 7.462 & 115.811 \\
\hline N60 & 8.015 & 116.248 & 8.105 & 116.202 & 8.189 & 116.145 & 8.270 & 116.072 & 8.324 & 116.017 \\
\hline $\mathrm{I} 61$ & 7.097 & 119.230 & 7.180 & 119.157 & 7.254 & 119.080 & 7.326 & 118.995 & 7.374 & 118.935 \\
\hline Q62 & 7.654 & 125.248 & 7.716 & 125.231 & 7.770 & 125.201 & 7.820 & 125.154 & 7.851 & 125.112 \\
\hline K63 & 8.414 & 120.625 & 8.461 & 120.614 & 8.500 & 120.591 & 8.535 & 120.548 & 8.555 & 120.513 \\
\hline E64 & 9.352 & 115.020 & 9.412 & 114.995 & 9.465 & 114.976 & 9.514 & 114.959 & 9.544 & 114.955 \\
\hline S65 & 7.582 & 115.506 & 7.657 & 115.359 & 7.729 & 115.217 & 7.800 & 115.072 & 7.848 & 114.973 \\
\hline T66 & 8.767 & 117.667 & 8.824 & 117. & 8.872 & 117.7 & 8.913 & 117.7 & 8.937 & 117.761 \\
\hline $\mathrm{L} 67$ & 9.161 & 127.748 & 9.254 & 127.749 & 9.340 & 127.737 & 9.422 & 127.711 & 9.477 & 127.689 \\
\hline $\mathrm{H} 68$ & 9.145 & 119.966 & 9.245 & 120.051 & 9.329 & 120.108 & 9.406 & 120.137 & 9.453 & 120.150 \\
\hline L69 & 8.004 & 123.832 & 8.111 & 123.903 & 8.213 & 123.981 & 8.315 & 124.061 & 8.383 & 124.118 \\
\hline V70 & 9.026 & 126.312 & 9.112 & 126.555 & 9.184 & 126.626 & 9.249 & 126.579 & 9.289 & 126.506 \\
\hline $\mathrm{L} 71$ & 8.059 & 122.860 & 8.091 & 122.923 & 8.117 & 122.964 & 8.138 & 122.986 & 8.150 & 122.994 \\
\hline $\mathrm{R} 72$ & 8.451 & 123.728 & 8.534 & 123.749 & 8.610 & 123.734 & 8.681 & 123.685 & 8.721 & 123.630 \\
\hline $\mathrm{L} 73$ & 8.361 & 125.127 & 8.384 & 124.900 & 8.401 & 124.667 & 8.415 & 124.426 & 8.424 & 124.263 \\
\hline $\mathrm{R} 74$ & 8.431 & 122.602 & 8.458 & 122.368 & 8.480 & 122.133 & 8.501 & 121.894 & 8.514 & 121.731 \\
\hline G75 & 8.466 & 111.595 & 8.493 & 111.387 & 8.518 & 111.191 & 8.543 & 111.010 & - & - \\
\hline G76 & 7.914 & 115.319 & 7.953 & 115.292 & 7.989 & 115.258 & 8.024 & 115.216 & 8.048 & 115.183 \\
\hline
\end{tabular}


Tab. 7.9: T12C Tm: Chemische Verschiebungen $\left({ }^{1} \mathrm{H}\right.$ und $\left.{ }^{15} \mathrm{~N}\right)$ der Amidsignale des Proteinrückgrats bei unterschiedlichen Temperaturen [ppm].

\begin{tabular}{|c|c|c|c|c|c|c|c|c|c|c|}
\hline \multirow[b]{2}{*}{ AS } & \multicolumn{2}{|c|}{$278 \mathrm{~K}$} & \multicolumn{2}{|c|}{$288 \mathrm{~K}$} & \multicolumn{2}{|c|}{$298 \mathrm{~K}$} & \multicolumn{2}{|c|}{$308 \mathrm{~K}$} & \multicolumn{2}{|c|}{$315 \mathrm{~K}$} \\
\hline & ${ }^{1} \mathrm{H}$ & ${ }^{15} \mathrm{~N}$ & ${ }^{1} \mathrm{H}$ & ${ }^{15} \mathrm{~N}$ & ${ }^{1} \mathrm{H}$ & ${ }^{15} \mathrm{~N}$ & ${ }^{1} \mathrm{H}$ & ${ }^{15} \mathrm{~N}$ & ${ }^{1} \mathrm{H}$ & ${ }^{15} \mathrm{~N}$ \\
\hline Q2 & 8.940 & 123.406 & 8.966 & 123.443 & 8.987 & 123.488 & 9.006 & 123.542 & 9.016 & 123.578 \\
\hline I3 & 8.136 & 115.609 & 8.220 & 115.693 & 8.302 & 115.791 & 8.383 & 115.901 & 8.436 & 115.978 \\
\hline $\mathrm{F} 4$ & 8.508 & 117.642 & 8.536 & 117.765 & 8.578 & 117.882 & 8.626 & 118.004 & 8.659 & 118.088 \\
\hline V5 & 8.416 & 120.101 & 8.525 & 120.157 & 8.629 & 120.213 & 8.733 & 120.276 & 8.802 & 120.325 \\
\hline $\mathrm{K} 6$ & - & - & 8.543 & 128.662 & 8.620 & 128.677 & 8.713 & 128.680 & 8.776 & 128.676 \\
\hline $\mathrm{T} 7$ & 7.528 & 117.221 & 7.688 & 117.118 & 7.843 & 116.999 & 7.997 & 116.864 & 8.098 & 116.766 \\
\hline L8 & 8.471 & 120.632 & 8.517 & 120.485 & 8.569 & 120.370 & 8.624 & 120.281 & 8.660 & 120.239 \\
\hline T9 & 6.649 & 105.297 & 6.852 & 105.386 & 7.045 & 105.467 & 7.228 & 105.539 & 7.346 & 105.586 \\
\hline G10 & 6.325 & 108.844 & 6.623 & 108.952 & 6.887 & 109.037 & 7.137 & 109.099 & 7.297 & 109.126 \\
\hline $\mathrm{K} 11$ & 5.921 & 120.496 & 6.162 & 120.451 & 6.389 & 120.404 & 6.602 & 120.343 & 6.738 & 120.294 \\
\hline $\mathrm{C} 12$ & - & - & - & - & - & - & - & - & - & - \\
\hline $\mathrm{I} 13$ & - & - & 8.482 & 123.955 & 8.576 & 123.609 & 8.699 & 123.594 & 8.763 & 123.494 \\
\hline $\mathrm{T} 14$ & 7.980 & 120.042 & 8.037 & 119.976 & 8.085 & 119.877 & 8.127 & 119.759 & 8.152 & 119.675 \\
\hline L15 & 8.424 & 124.795 & 8.499 & 124.842 & 8.569 & 124.860 & 8.637 & 124.860 & 8.681 & 124.85 \\
\hline E16 & 7.951 & 122.650 & 8.027 & 122.667 & 8.093 & 122.675 & 8.154 & 122.676 & 8.190 & 122.671 \\
\hline V17 & 8.970 & 117.839 & 9.016 & 117.850 & 9.058 & 117.848 & 9.098 & 117.836 & 9.124 & 117.821 \\
\hline E18 & 8.694 & 119.283 & 8.783 & 119.460 & 8.866 & 119.628 & 8.947 & 119.792 & 8.998 & 119.899 \\
\hline $\mathrm{S} 20$ & 7.220 & 103.647 & 7.259 & 103.715 & 7.301 & 103.788 & 7.345 & 103.871 & 7.376 & 103.931 \\
\hline D21 & 8.293 & 124.662 & 8.318 & 124.502 & 8.344 & 124.344 & 8.372 & 124.187 & 8.391 & 124.081 \\
\hline $\mathrm{T} 22$ & 8.065 & 109.545 & 8.079 & 109.410 & 8.098 & 109.284 & 8.122 & 109.163 & 8.139 & 109.084 \\
\hline $\mathrm{I} 23$ & 8.772 & 121.686 & 8.798 & 121.687 & 8.826 & 121.685 & 8.857 & 121.682 & 8.878 & 121.68 \\
\hline $\mathrm{N} 25$ & 8.042 & 121.872 & 8.081 & 121.769 & 8.121 & 121.650 & 8.163 & 121.517 & 8.191 & 121.420 \\
\hline V26 & 8.256 & 122.716 & 8.286 & 122.607 & 8.319 & 122.495 & 8.355 & 122.382 & 8.380 & 122.302 \\
\hline $\mathrm{K} 27$ & 8.672 & 119.506 & 8.710 & 119.396 & 8.750 & 119.288 & 8.792 & 119.180 & 8.820 & 119.106 \\
\hline $\mathrm{A} 28$ & 7.916 & 123.831 & 7.990 & 123.731 & 8.063 & 123.629 & 8.137 & 123.523 & 8.187 & 123.450 \\
\hline K29 & 7.796 & 120.566 & 7.864 & 120.480 & 7.933 & 120.387 & 8.004 & 120.286 & 8.052 & 120.211 \\
\hline I30 & 8.270 & 121.881 & 8.320 & 121.716 & 8.372 & 121.564 & 8.426 & 121.416 & 8.462 & 121.318 \\
\hline Q31 & 8.415 & 123.711 & 8.496 & 123.693 & 8.576 & 123.710 & 8.649 & 123.627 & 8.697 & 123.598 \\
\hline D32 & 7.882 & 119.836 & 7.934 & 119.784 & 7.989 & 119.735 & 8.048 & 119.688 & 8.088 & 119.657 \\
\hline K33 & 7.184 & 115.765 & 7.291 & 115.727 & 7.397 & 115.688 & 7.503 & 115.647 & 7.574 & 115.618 \\
\hline E34 & 8.512 & 114.480 & 8.605 & 114.514 & 8.691 & 114.558 & 8.774 & 114.610 & 8.827 & 114.645 \\
\hline G35 & 8.262 & 109.026 & 8.345 & 108.945 & 8.425 & 108.861 & 8.504 & 108.772 & 8.556 & 108.708 \\
\hline I36 & 5.911 & 120.687 & 6.020 & 120.531 & 6.127 & 120.384 & 6.235 & 120.240 & 6.307 & 120.142 \\
\hline D39 & 8.512 & 114.097 & 8.563 & 113.936 & 8.616 & 113.801 & 8.672 & 113.681 & 8.712 & 113.609 \\
\hline $\mathrm{Q} 40$ & 7.766 & 117.080 & 7.835 & 117.071 & 7.901 & 117.041 & 7.968 & 116.995 & 8.013 & 116.954 \\
\hline Q41 & 7.517 & 118.784 & 7.553 & 118.552 & 7.593 & 118.372 & 7.639 & 118.224 & 7.671 & 118.137 \\
\hline $\mathrm{R} 42$ & 8.506 & 123.781 & 8.648 & 123.673 & 8.713 & 123.502 & 8.770 & 123.357 & 8.804 & 123.268 \\
\hline L43 & 9.296 & 125.293 & 9.260 & 125.076 & 9.234 & 124.919 & 9.216 & 124.805 & 9.206 & 124.747 \\
\hline $\mathrm{I} 44$ & - & - & - & - & 10.166 & 123.682 & 10.062 & 123.601 & 10.001 & 123.535 \\
\hline F45 & - & - & 10.506 & 126.623 & 10.349 & 126.476 & 10.200 & 126.328 & 10.108 & 126.229 \\
\hline
\end{tabular}


Tab. 7.9: Fortsetzung.

\begin{tabular}{|c|c|c|c|c|c|c|c|c|c|c|}
\hline \multirow[b]{2}{*}{$\mathrm{AS}$} & \multicolumn{2}{|c|}{$278 \mathrm{~K}$} & \multicolumn{2}{|c|}{$288 \mathrm{~K}$} & \multicolumn{2}{|c|}{$298 \mathrm{~K}$} & \multicolumn{2}{|c|}{$308 \mathrm{~K}$} & \multicolumn{2}{|c|}{$315 \mathrm{~K}$} \\
\hline & ${ }^{1} \mathrm{H}$ & ${ }^{15} \mathrm{~N}$ & ${ }^{1} \mathrm{H}$ & ${ }^{15} \mathrm{~N}$ & ${ }^{1} \mathrm{H}$ & ${ }^{15} \mathrm{~N}$ & ${ }^{1} \mathrm{H}$ & ${ }^{15} \mathrm{~N}$ & ${ }^{1} \mathrm{H}$ & ${ }^{15} \mathrm{~N}$ \\
\hline A 46 & - & - & - & - & - & - & - & - & - & - \\
\hline G47 & - & - & - & - & - & - & - & - & - & - \\
\hline K48 & - & - & 9.563 & 123.529 & 9.440 & 123.246 & 9.331 & 122.961 & 9.263 & 122.768 \\
\hline Q49 & 9.361 & 123.889 & 9.309 & 123.734 & 9.257 & 123.564 & 9.206 & 123.388 & 9.172 & 123.268 \\
\hline L50 & 9.353 & 126.848 & 9.307 & 126.725 & 9.266 & 126.595 & 9.229 & 126.456 & 9.204 & 126.358 \\
\hline $\mathrm{E} 51$ & 8.876 & 123.884 & 8.849 & 123.807 & 8.827 & 123.731 & 8.808 & 123.656 & 8.795 & 123.605 \\
\hline D52 & 8.442 & 121.128 & 8.439 & 121.012 & 8.440 & 120.895 & 8.444 & 120.776 & 8.448 & 120.696 \\
\hline $\mathrm{R} 54$ & 7.722 & 120.231 & 7.751 & 120.039 & 7.784 & 119.849 & 7.819 & 119.656 & 7.844 & 119.525 \\
\hline $\mathrm{T} 55$ & 9.111 & 109.268 & 9.146 & 109.250 & 9.179 & 109.229 & 9.212 & 109.209 & 9.233 & 109.196 \\
\hline L56 & 8.492 & 118.678 & 8.513 & 118.649 & 8.538 & 118.628 & 8.567 & 118.617 & 8.588 & 118.614 \\
\hline $\mathrm{S} 57$ & 8.987 & 114.359 & 8.963 & 114.208 & 8.944 & 114.060 & 8.929 & 113.912 & 8.921 & 113.814 \\
\hline D58 & 8.392 & 125.358 & 8.394 & 125.231 & 8.398 & 125.090 & 8.404 & 124.938 & 8.408 & 124.830 \\
\hline Y59 & 7.932 & 116.927 & 7.915 & 116.735 & 7.905 & 116.553 & 7.900 & 116.373 & 7.899 & 116.255 \\
\hline N60 & 8.971 & 117.431 & 8.930 & 117.223 & 8.896 & 117.021 & 8.868 & 116.817 & 8.852 & 116.68 \\
\hline $\mathrm{I} 61$ & 8.303 & 120.695 & 8.221 & 120.420 & 8.146 & 120.158 & 8.078 & 119.903 & 8.037 & 119.737 \\
\hline Q62 & 9.661 & 126.882 & 9.437 & 126.649 & 9.244 & 126.416 & 9.064 & 126.181 & 8.951 & 126.022 \\
\hline K63 & 9.323 & 121.594 & 9.242 & 121.445 & 9.169 & 121.297 & 9.100 & 121.145 & 9.056 & 121.043 \\
\hline E64 & 9.685 & 115.487 & 9.669 & 115.363 & 9.656 & 115.252 & 9.648 & 115.162 & 9.645 & 115.114 \\
\hline S65 & 8.591 & 116.551 & 8.485 & 116.207 & 8.398 & 115.898 & 8.331 & 115.613 & 8.295 & 115.434 \\
\hline T66 & - & - & - & - & - & - & - & - & - & - \\
\hline $\mathrm{L} 67$ & - & - & - & - & 9.476 & 128.116 & 9.497 & 127.983 & 9.509 & 127.895 \\
\hline $\mathrm{H} 68$ & - & - & - & - & - & - & - & - & - & - \\
\hline L69 & - & - & 8.186 & 124.378 & 8.242 & 124.314 & 8.285 & 124.309 & 8.322 & 124.317 \\
\hline V70 & - & - & 9.474 & 126.950 & 9.466 & 126.921 & 9.465 & 126.796 & 9.468 & 126.68 \\
\hline $\mathrm{L} 71$ & 8.046 & 122.983 & 8.070 & 123.014 & 8.093 & 123.030 & 8.116 & 123.036 & 8.130 & 123.035 \\
\hline $\mathrm{R} 72$ & 8.576 & 123.889 & 8.578 & 123.792 & 8.646 & 123.772 & 8.712 & 123.720 & 8.754 & 123.667 \\
\hline L73 & 8.390 & 125.150 & 8.407 & 124.922 & 8.422 & 124.689 & 8.435 & 124.452 & 8.443 & 124.292 \\
\hline R74 & 8.403 & 122.583 & 8.439 & 122.357 & 8.469 & 122.128 & 8.497 & 121.895 & 8.514 & 121.735 \\
\hline G75 & 8.445 & 111.580 & 8.480 & 111.381 & 8.512 & 111.191 & 8.544 & 111.003 & - & - \\
\hline G76 & 7.901 & 115.299 & 7.945 & 115.278 & 7.985 & 115.248 & 7.981 & 115.225 & 8.050 & 115.184 \\
\hline
\end{tabular}


Tab. 7.10: T12C Tm: PCSs $\left({ }^{1} \mathrm{H}\right.$ und $\left.{ }^{15} \mathrm{~N}\right)$ der Amidsignale des Proteinrückgrats [ppm] in Abhängigkeit von der Temperatur. Als Referenz diente die diamagnetische Probe T12C Lu. Die zugrunde liegenden chemischen Verschiebungen sind den Tabellen 7.8 und 7.9 zu entnehmen.

\begin{tabular}{|c|c|c|c|c|c|c|c|c|c|c|}
\hline \multirow[b]{2}{*}{$\mathrm{AS}$} & \multicolumn{2}{|c|}{$278 \mathrm{~K}$} & \multicolumn{2}{|c|}{$288 \mathrm{~K}$} & \multicolumn{2}{|c|}{$298 \mathrm{~K}$} & \multicolumn{2}{|c|}{$308 \mathrm{~K}$} & \multicolumn{2}{|c|}{$315 \mathrm{~K}$} \\
\hline & ${ }^{1} \mathrm{H}$ & ${ }^{15} \mathrm{~N}$ & ${ }^{1} \mathrm{H}$ & ${ }^{15} \mathrm{~N}$ & ${ }^{1} \mathrm{H}$ & ${ }^{15} \mathrm{~N}$ & ${ }^{1} \mathrm{H}$ & ${ }^{15} \mathrm{~N}$ & ${ }^{1} \mathrm{H}$ & ${ }^{15} \mathrm{~N}$ \\
\hline Q2 & 0.092 & 0.056 & 0.061 & 0.035 & 0.036 & 0.013 & 0.015 & 0.000 & 0.002 & -0.010 \\
\hline I3 & -0.032 & -0.024 & -0.064 & -0.057 & -0.089 & -0.085 & -0.111 & -0.105 & -0.125 & -0.117 \\
\hline $\mathrm{F} 4$ & -0.138 & -0.364 & -0.186 & -0.372 & -0.215 & -0.380 & -0.236 & -0.382 & -0.248 & -0.382 \\
\hline V5 & -0.665 & -0.572 & -0.646 & -0.568 & -0.625 & -0.561 & -0.603 & -0.545 & -0.588 & -0.533 \\
\hline $\mathrm{K} 6$ & - & - & -0.482 & -0.819 & -0.493 & -0.776 & -0.484 & -0.722 & -0.475 & -0.684 \\
\hline $\mathrm{T} 7$ & -1.252 & -1.096 & -1.147 & -0.997 & -1.044 & -0.898 & -0.944 & -0.793 & -0.879 & -0.722 \\
\hline L8 & -0.604 & -0.756 & -0.552 & -0.693 & -0.496 & -0.625 & -0.438 & -0.551 & -0.399 & -0.499 \\
\hline Т9 & -0.838 & -0.868 & -0.723 & -0.739 & -0.612 & -0.618 & -0.509 & -0.500 & -0.453 & -0.434 \\
\hline G10 & -1.514 & -1.439 & -1.293 & -1.214 & -1.102 & -1.011 & -0.924 & -0.817 & -0.812 & -0.699 \\
\hline K11 & -1.107 & -1.275 & -0.959 & -1.120 & -0.821 & -0.971 & -0.696 & -0.832 & -0.619 & -0.745 \\
\hline $\mathrm{C} 12$ & - & - & - & - & - & - & - & - & - & - \\
\hline I13 & - & - & -1.032 & -1.147 & -0.999 & -1.270 & -0.934 & -1.079 & -0.909 & -1.059 \\
\hline T14 & -0.647 & -0.629 & -0.628 & -0.616 & -0.611 & -0.607 & -0.597 & -0.597 & -0.590 & -0.590 \\
\hline L15 & -0.263 & -0.256 & -0.271 & -0.263 & -0.276 & -0.272 & -0.280 & -0.271 & -0.281 & -0.273 \\
\hline E16 & -0.030 & -0.047 & -0.044 & -0.061 & -0.055 & -0.072 & -0.064 & -0.078 & -0.070 & -0.082 \\
\hline V17 & 0.144 & 0.148 & 0.109 & 0.114 & 0.079 & 0.082 & 0.052 & 0.058 & 0.037 & 0.043 \\
\hline E18 & 0.246 & 0.227 & 0.202 & 0.189 & 0.165 & 0.156 & 0.132 & 0.129 & 0.110 & 0.112 \\
\hline S20 & 0.321 & 0.313 & 0.271 & 0.266 & 0.229 & 0.220 & 0.188 & 0.185 & 0.163 & 0.162 \\
\hline D21 & 0.332 & 0.313 & 0.279 & 0.265 & 0.233 & 0.220 & 0.191 & 0.184 & 0.164 & 0.162 \\
\hline $\mathrm{T} 22$ & 0.261 & 0.272 & 0.218 & 0.227 & 0.181 & 0.185 & 0.148 & 0.152 & 0.126 & 0.131 \\
\hline I 23 & 0.380 & 0.367 & 0.320 & 0.308 & 0.267 & 0.254 & 0.220 & 0.209 & 0.189 & 0.182 \\
\hline $\mathrm{N} 25$ & 0.232 & 0.160 & 0.191 & 0.128 & 0.156 & 0.099 & 0.125 & 0.078 & 0.105 & 0.064 \\
\hline V26 & 0.261 & 0.207 & 0.212 & 0.167 & 0.171 & 0.128 & 0.135 & 0.099 & 0.113 & 0.080 \\
\hline $\mathrm{K} 27$ & 0.232 & 0.202 & 0.186 & 0.162 & 0.148 & 0.125 & 0.115 & 0.097 & 0.093 & 0.079 \\
\hline A 28 & 0.137 & 0.086 & 0.108 & 0.066 & 0.083 & 0.046 & 0.063 & 0.035 & 0.050 & 0.030 \\
\hline K29 & 0.097 & 0.038 & 0.071 & 0.020 & 0.050 & 0.004 & 0.032 & -0.006 & 0.021 & -0.012 \\
\hline I30 & 0.050 & 0.021 & 0.026 & 0.001 & 0.009 & -0.015 & -0.006 & -0.026 & -0.016 & -0.031 \\
\hline Q31 & 0.020 & -0.004 & 0.004 & -0.016 & -0.004 & 0.022 & -0.015 & -0.028 & -0.024 & -0.032 \\
\hline D32 & -0.007 & -0.055 & -0.016 & -0.056 & -0.020 & -0.057 & -0.024 & -0.053 & -0.026 & -0.049 \\
\hline K33 & -0.071 & -0.095 & -0.073 & -0.091 & -0.071 & -0.087 & -0.067 & -0.079 & -0.065 & -0.073 \\
\hline E34 & -0.121 & -0.115 & -0.117 & -0.109 & -0.110 & -0.104 & -0.100 & -0.089 & -0.094 & -0.081 \\
\hline G35 & -0.089 & -0.088 & -0.084 & -0.081 & -0.076 & -0.074 & -0.068 & -0.061 & -0.063 & -0.054 \\
\hline I36 & -0.077 & -0.081 & -0.076 & -0.077 & -0.071 & -0.071 & -0.065 & -0.063 & -0.062 & -0.057 \\
\hline D39 & 0.071 & 0.036 & 0.056 & 0.027 & 0.044 & 0.016 & 0.033 & 0.014 & 0.026 & 0.012 \\
\hline Q40 & 0.058 & 0.054 & 0.045 & 0.041 & 0.035 & 0.030 & 0.026 & 0.028 & 0.020 & 0.025 \\
\hline Q41 & 0.112 & 0.133 & 0.088 & 0.107 & 0.069 & 0.086 & 0.053 & 0.072 & 0.043 & 0.064 \\
\hline $\mathrm{R} 42$ & 0.203 & 0.157 & 0.217 & 0.216 & 0.174 & 0.173 & 0.137 & 0.137 & 0.115 & 0.116 \\
\hline
\end{tabular}


Tab. 7.10: Fortsetzung.

\begin{tabular}{|c|c|c|c|c|c|c|c|c|c|c|}
\hline \multirow[b]{2}{*}{ AS } & \multicolumn{2}{|c|}{$278 \mathrm{~K}$} & \multicolumn{2}{|c|}{$288 \mathrm{~K}$} & \multicolumn{2}{|c|}{$298 \mathrm{~K}$} & \multicolumn{2}{|c|}{$308 \mathrm{~K}$} & \multicolumn{2}{|c|}{$315 \mathrm{~K}$} \\
\hline & ${ }^{1} \mathrm{H}$ & ${ }^{15} \mathrm{~N}$ & ${ }^{1} \mathrm{H}$ & ${ }^{15} \mathrm{~N}$ & ${ }^{1} \mathrm{H}$ & ${ }^{15} \mathrm{~N}$ & ${ }^{1} \mathrm{H}$ & ${ }^{15} \mathrm{~N}$ & ${ }^{1} \mathrm{H}$ & ${ }^{15} \mathrm{~N}$ \\
\hline $\mathrm{L} 43$ & 0.606 & 0.664 & 0.501 & 0.561 & 0.418 & 0.451 & 0.342 & 0.368 & 0.295 & 0.316 \\
\hline $\mathrm{I} 44$ & - & - & - & - & 0.997 & 0.957 & 0.808 & 0.778 & 0.693 & 0.671 \\
\hline F45 & - & - & 1.742 & 2.083 & 1.490 & 1.763 & 1.254 & 1.486 & 1.106 & 1.312 \\
\hline A 46 & - & - & - & - & - & - & - & - & - & - \\
\hline G47 & - & - & - & - & - & - & - & - & - & - \\
\hline K48 & - & - & 1.629 & 1.295 & 1.406 & 1.115 & 1.199 & 0.949 & 1.066 & 0.843 \\
\hline Q49 & 0.797 & 0.883 & 0.691 & 0.758 & 0.593 & 0.641 & 0.500 & 0.540 & 0.440 & 0.477 \\
\hline L50 & 0.886 & 0.867 & 0.751 & 0.737 & 0.635 & 0.623 & 0.530 & 0.522 & 0.463 & 0.459 \\
\hline E51 & 0.546 & 0.506 & 0.465 & 0.437 & 0.395 & 0.371 & 0.330 & 0.313 & 0.288 & 0.276 \\
\hline D52 & 0.325 & 0.342 & 0.273 & 0.292 & 0.229 & 0.245 & 0.190 & 0.203 & 0.164 & 0.179 \\
\hline $\mathrm{R} 54$ & 0.352 & 0.348 & 0.298 & 0.298 & 0.252 & 0.251 & 0.208 & 0.210 & 0.180 & 0.187 \\
\hline $\mathrm{T} 55$ & 0.429 & 0.381 & 0.365 & 0.321 & 0.309 & 0.266 & 0.256 & 0.221 & 0.222 & 0.192 \\
\hline $\mathrm{L} 56$ & 0.498 & 0.561 & 0.422 & 0.475 & 0.355 & 0.399 & 0.294 & 0.333 & 0.254 & 0.291 \\
\hline $\mathrm{S} 57$ & 0.565 & 0.629 & 0.483 & 0.538 & 0.409 & 0.455 & 0.341 & 0.382 & 0.298 & 0.338 \\
\hline D58 & 0.576 & 0.568 & 0.494 & 0.487 & 0.421 & 0.411 & 0.353 & 0.349 & 0.308 & 0.307 \\
\hline Y59 & 0.807 & 0.809 & 0.694 & 0.696 & 0.593 & 0.593 & 0.498 & 0.501 & 0.437 & 0.444 \\
\hline N60 & 0.956 & 1.183 & 0.825 & 1.021 & 0.707 & 0.876 & 0.598 & 0.745 & 0.528 & 0.663 \\
\hline I61 & 1.206 & 1.465 & 1.041 & 1.263 & 0.892 & 1.078 & 0.752 & 0.908 & 0.663 & 0.802 \\
\hline Q62 & 2.007 & 1.634 & 1.721 & 1.418 & 1.474 & 1.215 & 1.244 & 1.027 & 1.100 & 0.910 \\
\hline K63 & 0.909 & 0.969 & 0.781 & 0.831 & 0.669 & 0.706 & 0.565 & 0.597 & 0.501 & 0.530 \\
\hline E64 & 0.333 & 0.467 & 0.257 & 0.368 & 0.191 & 0.276 & 0.134 & 0.203 & 0.101 & 0.159 \\
\hline S65 & 1.009 & 1.045 & 0.828 & 0.848 & 0.669 & 0.681 & 0.531 & 0.541 & 0.447 & 0.461 \\
\hline T66 & - & - & - & - & - & - & - & - & - & - \\
\hline L67 & - & - & - & - & 0.136 & 0.379 & 0.075 & 0.272 & 0.032 & 0.206 \\
\hline H68 & - & - & - & - & - & - & - & - & - & - \\
\hline L69 & - & - & 0.075 & 0.475 & 0.029 & 0.333 & -0.030 & 0.248 & -0.061 & 0.199 \\
\hline V70 & - & - & 0.362 & 0.395 & 0.282 & 0.295 & 0.216 & 0.217 & 0.179 & 0.174 \\
\hline L71 & -0.013 & 0.123 & -0.021 & 0.091 & -0.024 & 0.066 & -0.022 & 0.050 & -0.020 & 0.041 \\
\hline $\mathrm{R} 72$ & 0.125 & 0.161 & 0.044 & 0.043 & 0.036 & 0.038 & 0.031 & 0.035 & 0.033 & 0.037 \\
\hline L73 & 0.029 & 0.023 & 0.023 & 0.022 & 0.021 & 0.022 & 0.020 & 0.026 & 0.019 & 0.029 \\
\hline R74 & -0.028 & -0.019 & -0.019 & -0.011 & -0.011 & -0.005 & -0.004 & 0.001 & 0.000 & 0.004 \\
\hline G75 & -0.021 & -0.015 & -0.013 & -0.006 & -0.006 & 0.000 & 0.001 & -0.007 & - & - \\
\hline G76 & -0.013 & -0.020 & -0.008 & -0.014 & -0.004 & -0.010 & -0.043 & 0.009 & 0.002 & 0.001 \\
\hline
\end{tabular}


Tab. 7.11: T12C: ${ }^{1} J$ N-H Kopplungen des Proteinrückgrats der isotropen und anisotropen Proben und deren experimentelle und zurück berechnete RDCs (PALES), mit den entsprechenden Abweichungen [Hz]. Die grau unterlegten Felder markieren RDCs, die auf Grund von überlappten oder schwachen Signalen, ebenso wie die drei C-terminalen Reste nicht in den Berechnungen berücksichtigt wurden.

\begin{tabular}{|c|c|c|c|c|c|c|c|c|c|}
\hline \multirow[b]{2}{*}{$\mathrm{AS}$} & \multirow[b]{2}{*}{ isotrop } & \multicolumn{2}{|c|}{ Terbium } & \multicolumn{6}{|c|}{ Thulium } \\
\hline & & anisotrop & RDC (exp) & RDC (ber) & $\Delta \mathrm{RDC}$ & anisotrop & RDC (exp) & RDC (ber) & $\Delta \mathrm{RDC}$ \\
\hline Q2 & -92.83 & -99.43 & -6.60 & -7.67 & 1.07 & -94.22 & -1.39 & -0.23 & -1.16 \\
\hline I3 & -92.15 & -86.93 & 5.22 & 4.97 & 0.25 & -98.42 & -6.27 & -5.76 & -0.51 \\
\hline $\mathrm{F} 4$ & -93.25 & -78.81 & 14.44 & 14.62 & -0.18 & -102.90 & -9.65 & -9.51 & -0.14 \\
\hline V5 & -92.15 & -74.34 & 17.81 & 17.15 & 0.66 & -102.08 & -9.93 & -9.85 & -0.08 \\
\hline K6 & -92.95 & - & - & - & - & -99.88 & -6.93 & -7.34 & 0.41 \\
\hline $\mathrm{T} 7$ & -94.05 & -82.73 & 11.32 & - & - & -98.87 & -4.82 & -2.19 & -2.63 \\
\hline L8 & -92.34 & -98.60 & -6.26 & -7.12 & 0.86 & -86.84 & 5.50 & 6.05 & -0.55 \\
\hline $\mathrm{T} 9$ & -92.34 & -98.01 & -5.67 & -6.36 & 0.69 & -86.02 & 6.32 & 8.16 & -1.85 \\
\hline G10 & -92.95 & - & - & - & - & -89.48 & 3.47 & 2.76 & 0.71 \\
\hline K11 & -93.25 & - & - & - & - & -95.23 & -1.98 & -1.74 & -0.24 \\
\hline $\mathrm{C} 12$ & -92.77 & - & - & - & - & - & - & - & - \\
\hline I13 & -92.70 & - & - & - & - & -101.97 & -9.27 & -9.66 & 0.39 \\
\hline $\mathrm{T} 14$ & -92.46 & -79.27 & 13.19 & 13.39 & -0.20 & -100.89 & -8.43 & -8.48 & 0.05 \\
\hline L15 & -93.49 & -87.20 & 6.29 & 7.33 & -1.04 & -99.15 & -5.66 & -6.54 & 0.88 \\
\hline E16 & -91.79 & -92.50 & -0.71 & -1.43 & 0.72 & -97.15 & -5.36 & -3.47 & -1.89 \\
\hline V17 & -93.07 & -99.88 & -6.81 & -7.02 & 0.21 & -91.85 & 1.22 & 1.84 & -0.62 \\
\hline E18 & -91.92 & -97.96 & -6.04 & -6.88 & 0.84 & -93.13 & -1.21 & -0.55 & -0.66 \\
\hline S20 & -91.49 & -81.19 & 10.30 & 10.18 & 0.12 & -98.88 & -7.39 & -7.35 & -0.04 \\
\hline D21 & -94.11 & -100.16 & -6.05 & -6.01 & -0.04 & -89.49 & 4.62 & 3.74 & 0.88 \\
\hline $\mathrm{T} 22$ & -93.07 & -87.30 & 5.77 & 7.71 & -1.94 & -96.14 & -3.07 & -4.80 & 1.73 \\
\hline $\mathrm{I} 23$ & -93.37 & -80.00 & 13.37 & 12.03 & 1.34 & -100.43 & -7.06 & -5.16 & -1.90 \\
\hline N25 & -93.99 & -89.48 & 4.51 & 4.75 & -0.24 & -97.78 & -3.79 & -3.66 & -0.13 \\
\hline V26 & -93.25 & -78.90 & 14.35 & 13.07 & 1.28 & -100.62 & -7.37 & -6.94 & -0.43 \\
\hline K27 & -93.62 & -86.66 & 6.96 & 7.36 & -0.40 & -94.69 & -1.07 & -1.15 & 0.08 \\
\hline A28 & -93.92 & -93.50 & 0.42 & 1.03 & -0.61 & -92.77 & 1.15 & 0.72 & 0.43 \\
\hline K29 & -93.61 & -83.46 & 10.15 & 9.81 & 0.34 & -100.43 & -6.82 & -6.78 & -0.04 \\
\hline I30 & -93.32 & -82.73 & 10.59 & 11.75 & -1.16 & -97.33 & -4.01 & -5.04 & 1.03 \\
\hline Q31 & -93.56 & -91.85 & 1.71 & 1.38 & 0.33 & -90.94 & 2.62 & 2.27 & 0.36 \\
\hline D32 & -93.68 & -92.13 & 1.55 & 1.97 & -0.42 & -94.23 & -0.55 & -1.33 & 0.78 \\
\hline K33 & -92.58 & -79.99 & 12.59 & 11.64 & 0.95 & -100.34 & -7.76 & -6.89 & -0.87 \\
\hline E34 & -90.33 & -85.93 & 4.40 & 4.64 & -0.24 & -89.48 & 0.85 & 0.67 & 0.18 \\
\hline G35 & -93.32 & -102.62 & -9.30 & -10.27 & 0.97 & -87.84 & 5.48 & 5.78 & -0.30 \\
\hline I36 & -90.76 & -98.70 & -7.94 & -7.30 & -0.64 & -92.03 & -1.27 & -1.03 & -0.24 \\
\hline D39 & -93.25 & -86.47 & 6.78 & 7.93 & -1.15 & -98.51 & -5.26 & -6.34 & 1.08 \\
\hline
\end{tabular}


Tab. 7.11: Fortsetzung.

\begin{tabular}{|c|c|c|c|c|c|c|c|c|c|}
\hline \multirow[b]{2}{*}{$\mathrm{AS}$} & \multirow[b]{2}{*}{ isotrop } & \multicolumn{2}{|c|}{ Terbium } & \multirow[b]{2}{*}{ RDC (ber) } & \multirow[b]{2}{*}{$\Delta \mathrm{RDC}$} & \multicolumn{2}{|c|}{ Thulium } & \multirow[b]{2}{*}{ RDC (ber) } & \multirow[b]{2}{*}{$\Delta \mathrm{RDC}$} \\
\hline & & anisotrop & RDC (exp) & & & anisotrop & $\mathrm{RDC}(\exp )$ & & \\
\hline Q40 & -92.03 & -79.09 & 12.94 & 12.23 & 0.71 & -99.70 & -7.67 & -7.52 & -0.15 \\
\hline Q41 & -93.49 & -101.88 & -8.39 & -8.21 & -0.18 & -82.46 & 11.03 & 10.20 & 0.83 \\
\hline $\mathrm{R} 42$ & -93.00 & -96.27 & -3.27 & -1.66 & -1.61 & -84.92 & 8.08 & 6.48 & 1.60 \\
\hline $\mathrm{L} 43$ & -92.83 & -94.96 & -2.13 & -3.88 & 1.75 & -85.56 & 7.27 & 7.73 & -0.46 \\
\hline I44 & -91.98 & - & - & - & - & -94.68 & -2.70 & -2.57 & -0.13 \\
\hline F45 & -92.58 & - & - & - & - & -97.51 & -4.93 & -4.66 & -0.27 \\
\hline G47 & -92.34 & - & - & - & - & - & - & - & - \\
\hline K48 & -92.94 & - & - & - & - & -98.97 & -6.03 & -5.00 & -1.03 \\
\hline Q49 & -92.77 & -77.99 & 14.78 & 15.77 & -0.99 & -100.98 & -8.21 & -9.49 & 1.28 \\
\hline L50 & -94.04 & -90.39 & 3.65 & 3.93 & -0.28 & -92.22 & 1.82 & 1.21 & 0.61 \\
\hline $\mathrm{E} 51$ & -92.52 & -96.51 & -3.99 & - & - & -95.78 & -3.26 & -2.10 & -1.16 \\
\hline D52 & -92.64 & -97.42 & -4.78 & -2.92 & -1.86 & -92.68 & -0.04 & -2.21 & 2.17 \\
\hline $\mathrm{R} 54$ & -92.83 & -100.88 & -8.05 & -9.02 & 0.97 & -86.20 & 6.63 & 7.38 & -0.75 \\
\hline $\mathrm{T} 55$ & -91.97 & -83.10 & 8.87 & 8.85 & 0.02 & -98.34 & -6.37 & -5.88 & -0.49 \\
\hline $\mathrm{L} 56$ & -94.16 & -83.28 & 10.88 & 10.24 & 0.64 & -100.06 & -5.90 & -5.97 & 0.07 \\
\hline $\mathrm{S} 57$ & -93.50 & -90.49 & 3.01 & 1.51 & 1.50 & -93.77 & -0.27 & 0.52 & -0.79 \\
\hline D58 & -94.65 & -90.86 & 3.79 & 3.53 & 0.26 & -99.52 & -4.87 & -4.87 & 0.00 \\
\hline Y59 & -92.28 & -76.99 & 15.29 & 14.80 & 0.49 & -101.52 & -9.24 & -9.36 & 0.11 \\
\hline N60 & -93.56 & -103.17 & -9.61 & -8.53 & -1.08 & -82.65 & 10.91 & 10.87 & 0.04 \\
\hline I61 & -92.71 & -101.43 & -8.72 & -7.36 & -1.36 & -84.10 & 8.61 & 6.96 & 1.65 \\
\hline Q62 & -93.01 & -97.42 & -4.41 & -4.09 & -0.32 & -87.11 & 5.90 & 5.87 & 0.03 \\
\hline K63 & -93.07 & -103.54 & -10.47 & -9.30 & -1.17 & -88.30 & 4.77 & 4.70 & 0.07 \\
\hline E64 & -92.65 & -90.85 & 1.80 & 2.05 & -0.25 & -93.68 & -1.03 & -1.35 & 0.32 \\
\hline S65 & -93.68 & - & - & - & - & - & - & - & - \\
\hline T66 & -91.36 & - & - & - & - & - & - & - & - \\
\hline $\mathrm{L} 67$ & -92.89 & - & - & - & - & -106.18 & -13.29 & -10.28 & -3.01 \\
\hline H68 & -93.19 & - & - & - & - & - & - & - & - \\
\hline L69 & -92.95 & - & - & - & - & -89.45 & 3.50 & 4.05 & -0.55 \\
\hline V70 & -93.01 & -101.15 & -8.14 & -8.15 & 0.01 & -83.83 & 9.18 & 10.84 & -1.66 \\
\hline $\mathrm{L} 71$ & -92.95 & -97.42 & -4.47 & - & - & -84.37 & 8.58 & 9.87 & -1.29 \\
\hline $\mathrm{R} 72$ & -92.77 & -89.57 & 3.20 & 1.63 & 1.57 & -93.41 & -0.64 & 1.06 & -1.70 \\
\hline L73 & -93.07 & -88.93 & 4.14 & 5.67 & -1.53 & -94.68 & -1.61 & -2.49 & 0.88 \\
\hline
\end{tabular}


Tab. 7.12: S57C: ${ }^{1} J$ N-H Kopplungen des Proteinrückgrats der isotropen und anisotropen Proben und deren experimentelle und die berechnete RDCs (PALES) mit den entsprechenden Abweichungen $[\mathrm{Hz}]$. Die grau unterlegten Felder markieren RDCs, die auf Grund von überlappten oder schwachen Signalen, ebenso wie die drei C-terminalen Reste nicht in den Berechnungen berücksichtigt wurden.

\begin{tabular}{|c|c|c|c|c|c|c|c|c|c|}
\hline \multirow[b]{2}{*}{$\mathrm{AS}$} & \multirow[b]{2}{*}{ isotrop } & \multicolumn{2}{|c|}{ Terbium } & \multirow[b]{2}{*}{ RDC (ber) } & \multirow[b]{2}{*}{$\Delta \mathrm{RDC}$} & \multicolumn{2}{|c|}{ Thulium } & \multirow[b]{2}{*}{ RDC (ber) } & \multirow[b]{2}{*}{$\Delta \mathrm{RDC}$} \\
\hline & & anisotrop & $\mathrm{RDC}(\exp )$ & & & anisotrop & $\mathrm{RDC}(\exp )$ & & \\
\hline $\mathrm{Q} 2$ & -93.48 & - & - & - & - & - & - & - & - \\
\hline I3 & -92.12 & - & - & - & - & - & - & - & - \\
\hline $\mathrm{F} 4$ & -93.48 & -95.30 & -1.82 & -2.16 & 0.34 & - & - & - & - \\
\hline V5 & -92.82 & -94.56 & -1.74 & -2.07 & 0.33 & -93.13 & -0.31 & - & - \\
\hline K6 & -93.39 & -98.57 & -5.18 & -4.68 & -0.50 & -94.78 & -1.39 & -2.66 & 1.27 \\
\hline $\mathrm{T} 7$ & -94.07 & -97.30 & -3.23 & -4.30 & 1.07 & -89.76 & 4.31 & 4.76 & -0.45 \\
\hline L8 & -92.90 & -93.92 & -1.02 & -1.48 & 0.46 & -88.12 & 4.78 & 4.09 & 0.69 \\
\hline T9 & -91.99 & -89.09 & 2.90 & 2.80 & 0.10 & -93.22 & -1.23 & -0.81 & -0.42 \\
\hline G10 & -91.68 & -99.03 & -7.35 & -6.82 & -0.53 & -90.12 & 1.56 & 3.23 & -1.67 \\
\hline K11 & -93.24 & -93.01 & 0.23 & 0.33 & -0.10 & -87.57 & 5.67 & 5.74 & -0.07 \\
\hline $\mathrm{T} 12$ & -92.23 & -97.20 & -4.97 & -5.00 & 0.03 & -89.12 & 3.11 & 1.29 & 1.82 \\
\hline $\mathrm{I} 13$ & -92.78 & -94.10 & -1.32 & -1.12 & -0.20 & -93.23 & -0.45 & -0.81 & 0.36 \\
\hline $\mathrm{T} 14$ & -92.83 & -95.95 & -3.12 & -3.48 & 0.36 & -97.88 & -5.05 & -3.94 & -1.11 \\
\hline L15 & -93.51 & -93.74 & -0.23 & 0.12 & -0.35 & - & - & - & - \\
\hline E16 & -93.19 & - & - & - & - & - & - & - & - \\
\hline V17 & -93.68 & - & - & - & - & - & - & - & - \\
\hline E18 & -91.86 & - & - & - & - & - & - & - & - \\
\hline $\mathrm{S} 20$ & -92.31 & - & - & - & - & - & - & - & - \\
\hline D21 & -93.95 & - & - & - & - & - & - & - & - \\
\hline $\mathrm{T} 22$ & -93.65 & - & - & - & - & - & - & - & - \\
\hline $\mathrm{I} 23$ & -93.71 & -99.62 & -5.91 & -5.74 & -0.17 & - & - & - & - \\
\hline N25 & -94.09 & - & - & - & - & - & - & - & - \\
\hline V26 & -93.19 & - & - & - & - & -90.67 & 2.52 & - & - \\
\hline $\mathrm{K} 27$ & -94.47 & -100.49 & -6.02 & -6.78 & 0.76 & -94.32 & 0.15 & -0.07 & 0.22 \\
\hline A 28 & -94.36 & -100.13 & -5.77 & -5.33 & -0.44 & -96.96 & -2.60 & -3.03 & 0.43 \\
\hline K29 & -93.58 & -98.21 & -4.63 & -4.04 & -0.59 & -98.70 & -5.12 & -4.27 & -0.85 \\
\hline I30 & -93.21 & -99.38 & -6.17 & -5.79 & -0.38 & -93.96 & -0.75 & -1.19 & 0.44 \\
\hline Q31 & -93.52 & -100.76 & -7.24 & -6.26 & -0.98 & -95.32 & -1.80 & -1.53 & -0.27 \\
\hline D32 & -93.72 & -99.21 & -5.49 & -4.68 & -0.81 & -97.33 & -3.61 & -3.84 & 0.23 \\
\hline K33 & -93.10 & -97.39 & -4.29 & -4.60 & 0.31 & -95.96 & -2.86 & -3.59 & 0.73 \\
\hline E34 & -90.94 & -97.30 & -6.36 & -6.83 & 0.47 & -91.03 & -0.09 & -0.52 & 0.43 \\
\hline G35 & -94.40 & -90.10 & 4.30 & 1.81 & 2.48 & -96.51 & -2.11 & -2.88 & 0.77 \\
\hline I36 & -90.94 & -89.18 & 1.76 & 2.23 & -0.47 & -86.84 & 4.10 & 3.03 & 1.07 \\
\hline D39 & -93.76 & -92.73 & 1.03 & 0.74 & 0.29 & -96.42 & -2.66 & -3.62 & 0.96 \\
\hline
\end{tabular}


Tab. 7.12: Fortsetzung.

\begin{tabular}{|c|c|c|c|c|c|c|c|c|c|}
\hline \multirow[b]{2}{*}{$\mathrm{AS}$} & \multirow[b]{2}{*}{ isotrop } & \multicolumn{2}{|c|}{ Terbium } & \multirow[b]{2}{*}{ RDC (ber) } & \multirow[b]{2}{*}{$\Delta \mathrm{RDC}$} & \multicolumn{2}{|c|}{ Thulium } & \multirow[b]{2}{*}{ RDC (ber) } & \multirow[b]{2}{*}{$\Delta \mathrm{RDC}$} \\
\hline & & anisotrop & $\mathrm{RDC}(\exp )$ & & & anisotrop & RDC (exp) & & \\
\hline Q40 & -91.59 & -87.18 & 4.41 & 4.85 & -0.44 & -93.68 & -2.09 & -2.11 & 0.02 \\
\hline Q41 & -92.93 & -93.19 & -0.26 & -0.49 & 0.23 & -91.68 & 1.25 & 1.59 & -0.34 \\
\hline $\mathrm{R} 42$ & -92.89 & -99.21 & -6.32 & -6.54 & 0.22 & -93.86 & -0.97 & - & - \\
\hline $\mathrm{L} 43$ & -89.61 & -99.03 & -9.42 & - & - & -89.67 & -0.06 & - & - \\
\hline $\mathrm{I} 44$ & -92.67 & -99.03 & -6.36 & -6.41 & 0.05 & -91.31 & 1.36 & 0.80 & 0.56 \\
\hline F45 & -95.36 & -96.84 & -1.48 & - & - & -89.21 & 6.15 & - & - \\
\hline A 46 & -93.67 & -99.03 & -5.36 & -5.48 & 0.12 & -91.86 & 1.81 & 1.82 & -0.01 \\
\hline G47 & -92.74 & -97.76 & -5.02 & -4.07 & -0.95 & -97.14 & -4.40 & -2.81 & -1.59 \\
\hline K48 & -92.41 & -89.00 & 3.41 & 3.79 & -0.38 & -88.94 & 3.47 & 2.65 & 0.82 \\
\hline Q49 & -93.27 & -91.64 & 1.63 & 1.00 & 0.63 & -94.05 & -0.78 & -0.33 & -0.45 \\
\hline L50 & -93.38 & -100.67 & -7.29 & -6.82 & -0.47 & -93.50 & -0.12 & -0.51 & 0.39 \\
\hline E51 & -91.96 & -83.71 & 8.25 & - & - & - & - & - & - \\
\hline D52 & -92.61 & -88.18 & 4.43 & 5.30 & -0.87 & -92.40 & 0.21 & 1.37 & -1.16 \\
\hline $\mathrm{R} 54$ & -94.15 & -94.11 & 0.04 & -1.17 & 1.21 & - & & & \\
\hline $\mathrm{T} 55$ & -92.45 & - & - & - & - & - & - & - & - \\
\hline L56 & -94.25 & - & - & - & - & - & - & - & - \\
\hline C57 & -94.37 & - & - & - & - & - & - & - & - \\
\hline D58 & -93.85 & - & - & - & - & - & - & - & - \\
\hline Y59 & -93.61 & -90.18 & 3.43 & 1.31 & 2.12 & -91.67 & 1.94 & - & - \\
\hline N60 & -93.69 & -95.38 & -1.69 & -2.15 & 0.46 & -90.40 & 3.29 & 2.43 & 0.86 \\
\hline $\mathrm{I} 61$ & -92.97 & -95.02 & -2.05 & -2.41 & 0.36 & - & - & - & - \\
\hline Q62 & -92.99 & -89.09 & 3.90 & 4.44 & -0.54 & - & - & - & - \\
\hline K63 & -93.32 & - & - & - & - & - & - & - & - \\
\hline E64 & -93.64 & - & - & - & - & - & - & - & - \\
\hline $\mathrm{S} 65$ & -94.38 & - & - & - & - & - & - & - & - \\
\hline T66 & -91.74 & -91.55 & 0.19 & 0.32 & -0.13 & -97.15 & -5.41 & -4.12 & -1.29 \\
\hline $\mathrm{L} 67$ & -93.52 & -92.83 & 0.69 & 0.75 & -0.05 & -95.96 & -2.44 & -3.21 & 0.77 \\
\hline H68 & -92.81 & -99.39 & -6.58 & -6.53 & -0.05 & -92.67 & 0.14 & 0.12 & 0.02 \\
\hline L69 & -93.02 & -98.85 & -5.83 & -6.40 & 0.57 & -89.21 & 3.81 & 4.54 & -0.73 \\
\hline V70 & -93.55 & -98.12 & -4.57 & -3.41 & -1.16 & -93.22 & 0.33 & 1.14 & -0.81 \\
\hline $\mathrm{L} 71$ & -93.33 & -94.01 & -0.68 & 0.27 & -0.95 & -91.31 & 2.02 & 1.01 & 1.01 \\
\hline $\mathrm{R} 72$ & -93.51 & -88.63 & 4.88 & 5.68 & -0.80 & -95.96 & -2.45 & -2.55 & 0.10 \\
\hline L73 & -92.91 & -92.83 & 0.08 & - & - & -95.50 & -2.59 & -2.83 & 0.24 \\
\hline
\end{tabular}


Tab. 7.13: ${ }^{1} \mathrm{H},{ }^{15} \mathrm{~N}-\mathrm{PCS}$ von $\mathrm{T} 12 \mathrm{C}$ Tb und T12C Tm $[\mathrm{ppm}]$, berechnet im RDC-Tensor. Zusätzlich sind die Abweichungen $\Delta$ von den experimentellen Werten gegeben (siehe Tabellen 7.2 und 7.3).

\begin{tabular}{|c|c|c|c|c|c|c|c|c|}
\hline \multirow[b]{2}{*}{ AS } & \multicolumn{4}{|c|}{$\mathrm{T} 12 \mathrm{C} \mathrm{Tb}$} & \multicolumn{4}{|c|}{$\mathrm{T} 12 \mathrm{C} \mathrm{Tm}$} \\
\hline & ${ }^{1} \mathrm{H}$ (ber) & $\Delta{ }^{1} \mathrm{H}$ & ${ }^{15} \mathrm{~N}$ (ber) & $\Delta^{15} \mathrm{~N}$ & ${ }^{1} \mathrm{H}$ (ber) & $\Delta{ }^{1} \mathrm{H}$ & ${ }^{15} \mathrm{~N}$ (ber) & $\Delta^{15} \mathrm{~N}$ \\
\hline $\mathrm{Q} 2$ & 0.007 & -0.229 & 0.011 & -0.167 & 0.063 & 0.027 & 0.072 & 0.059 \\
\hline I3 & 0.102 & -0.123 & 0.082 & -0.145 & 0.003 & 0.093 & 0.037 & 0.121 \\
\hline $\mathrm{F} 4$ & 0.199 & 0.193 & 0.281 & 0.107 & -0.002 & 0.213 & -0.165 & 0.214 \\
\hline V5 & 0.621 & 0.220 & 0.591 & 0.338 & -0.519 & 0.106 & -0.459 & 0.103 \\
\hline K6 & - & - & - & - & -0.717 & -0.224 & -0.857 & -0.081 \\
\hline $\mathrm{T} 7$ & 1.481 & 0.241 & 1.316 & 0.174 & -0.891 & 0.153 & -0.790 & 0.108 \\
\hline $\mathrm{L} 8$ & 0.512 & -0.260 & 0.747 & -0.325 & -0.371 & 0.125 & -0.456 & 0.170 \\
\hline $\mathrm{T} 9$ & 0.820 & -0.180 & 0.868 & -0.191 & -0.435 & 0.177 & -0.428 & 0.190 \\
\hline G10 & 1.635 & -0.108 & 1.643 & 0.047 & -0.700 & 0.403 & -0.680 & 0.332 \\
\hline K11 & - & - & - & - & -0.721 & 0.101 & -0.807 & 0.164 \\
\hline $\mathrm{C} 12$ & - & - & - & - & - & - & - & - \\
\hline $\mathrm{I} 13$ & 0.859 & -0.134 & 0.815 & -0.467 & -0.705 & 0.289 & -0.677 & 0.471 \\
\hline $\mathrm{T} 14$ & 0.458 & -0.399 & 0.468 & -0.371 & -0.401 & 0.210 & -0.416 & 0.192 \\
\hline L15 & 0.236 & -0.130 & 0.231 & -0.131 & -0.166 & 0.110 & -0.164 & 0.108 \\
\hline E16 & 0.094 & -0.065 & 0.090 & -0.065 & -0.027 & 0.028 & -0.019 & 0.052 \\
\hline V17 & -0.005 & -0.024 & -0.007 & 0.031 & 0.108 & 0.029 & 0.106 & 0.024 \\
\hline E18 & -0.080 & 0.036 & -0.079 & 0.078 & 0.193 & 0.028 & 0.193 & 0.037 \\
\hline $\mathrm{S} 20$ & -0.128 & 0.054 & -0.141 & 0.034 & 0.242 & 0.013 & 0.264 & 0.044 \\
\hline D21 & -0.134 & 0.057 & -0.130 & 0.087 & 0.257 & 0.024 & 0.242 & 0.021 \\
\hline $\mathrm{T} 22$ & -0.120 & 0.043 & -0.138 & 0.012 & 0.202 & 0.021 & 0.232 & 0.047 \\
\hline $\mathrm{I} 23$ & -0.206 & 0.046 & -0.197 & 0.022 & 0.300 & 0.033 & 0.291 & 0.036 \\
\hline $\mathrm{N} 25$ & -0.120 & 0.030 & -0.107 & -0.009 & 0.178 & 0.022 & 0.159 & 0.059 \\
\hline V26 & -0.119 & 0.041 & -0.096 & 0.013 & 0.200 & 0.029 & 0.171 & 0.042 \\
\hline $\mathrm{K} 27$ & -0.116 & 0.028 & -0.092 & 0.024 & 0.167 & 0.019 & 0.142 & 0.017 \\
\hline A28 & -0.063 & 0.018 & -0.048 & -0.011 & 0.097 & 0.014 & 0.078 & 0.032 \\
\hline K29 & -0.015 & 0.020 & 0.002 & -0.027 & 0.064 & 0.014 & 0.043 & 0.038 \\
\hline I30 & 0.023 & 0.015 & 0.050 & 0.000 & 0.027 & 0.019 & 0.000 & 0.015 \\
\hline Q31 & 0.022 & 0.008 & 0.042 & 0.000 & 0.003 & 0.010 & -0.017 & 0.079 \\
\hline D32 & 0.046 & 0.016 & 0.057 & -0.014 & -0.018 & 0.002 & -0.029 & 0.029 \\
\hline K33 & 0.106 & 0.013 & 0.114 & 0.006 & -0.064 & 0.007 & -0.073 & 0.014 \\
\hline E34 & 0.136 & -0.002 & 0.154 & 0.070 & -0.093 & 0.017 & -0.108 & -0.005 \\
\hline G35 & 0.094 & 0.015 & 0.097 & 0.068 & -0.070 & 0.007 & -0.075 & -0.001 \\
\hline I36 & 0.070 & 0.000 & 0.052 & 0.021 & -0.063 & 0.008 & -0.055 & 0.016 \\
\hline D39 & -0.097 & -0.028 & -0.114 & -0.086 & 0.041 & -0.002 & 0.050 & 0.035 \\
\hline Q40 & -0.118 & -0.052 & -0.138 & -0.082 & 0.030 & -0.005 & 0.034 & 0.004 \\
\hline Q41 & -0.164 & -0.068 & -0.178 & -0.038 & 0.054 & -0.015 & 0.047 & -0.039 \\
\hline $\mathrm{R} 42$ & -0.326 & -0.108 & -0.340 & -0.076 & 0.092 & -0.082 & 0.120 & -0.053 \\
\hline $\mathrm{L} 43$ & -0.484 & -0.042 & -0.518 & -0.027 & 0.324 & -0.094 & 0.330 & -0.122 \\
\hline $\mathrm{I} 44$ & -0.928 & 0.146 & -1.006 & 0.008 & 0.719 & -0.278 & 0.781 & -0.176 \\
\hline
\end{tabular}


Tab. 7.13: Fortsetzung.

\begin{tabular}{|c|c|c|c|c|c|c|c|c|}
\hline \multirow[b]{2}{*}{$\mathrm{AS}$} & \multicolumn{4}{|c|}{$\mathrm{T} 12 \mathrm{C} \mathrm{Tb}$} & \multicolumn{4}{|c|}{$\mathrm{T} 12 \mathrm{C} \mathrm{Tm}$} \\
\hline & ${ }^{1} \mathrm{H}$ (ber) & $\Delta{ }^{1} \mathrm{H}$ & ${ }^{15} \mathrm{~N}$ (ber) & $\Delta{ }^{15} \mathrm{~N}$ & ${ }^{1} \mathrm{H}$ (ber) & $\Delta{ }^{1} \mathrm{H}$ & ${ }^{15} \mathrm{~N}$ (ber) & $\Delta{ }^{15} \mathrm{~N}$ \\
\hline F 45 & -1.579 & -0.227 & -1.697 & -0.247 & 1.657 & 0.167 & 1.943 & 0.180 \\
\hline A 46 & - & - & - & - & - & - & - & - \\
\hline G47 & - & - & - & - & - & - & - & - \\
\hline K48 & - & - & - & - & 1.661 & 0.255 & 1.229 & 0.114 \\
\hline Q49 & -0.766 & -0.243 & -0.878 & -0.296 & 0.526 & -0.067 & 0.635 & -0.007 \\
\hline L50 & -0.706 & -0.076 & -0.651 & -0.048 & 0.627 & -0.008 & 0.605 & -0.018 \\
\hline E51 & -0.383 & -0.016 & -0.376 & 0.011 & 0.433 & 0.039 & 0.400 & 0.029 \\
\hline D52 & -0.238 & -0.001 & -0.241 & 0.009 & 0.229 & 0.000 & 0.249 & 0.004 \\
\hline $\mathrm{R} 54$ & -0.223 & 0.015 & -0.209 & 0.058 & 0.284 & 0.033 & 0.275 & 0.024 \\
\hline T55 & -0.231 & 0.055 & -0.231 & 0.005 & 0.357 & 0.048 & 0.357 & 0.091 \\
\hline L56 & -0.234 & 0.083 & -0.267 & 0.089 & 0.417 & 0.063 & 0.473 & 0.073 \\
\hline S57 & -0.249 & 0.114 & -0.282 & 0.126 & 0.456 & 0.048 & 0.520 & 0.066 \\
\hline D58 & -0.283 & 0.099 & -0.300 & 0.067 & 0.486 & 0.066 & 0.517 & 0.106 \\
\hline Y59 & -0.405 & 0.142 & -0.425 & 0.115 & 0.713 & 0.121 & 0.738 & 0.146 \\
\hline N60 & -0.429 & 0.246 & -0.491 & 0.348 & 0.786 & 0.079 & 0.922 & 0.047 \\
\hline I61 & -0.486 & 0.324 & -0.537 & 0.460 & 0.995 & 0.103 & 1.137 & 0.060 \\
\hline Q62 & -0.569 & 0.656 & -0.479 & 0.525 & 1.394 & -0.079 & 1.128 & -0.087 \\
\hline K63 & -0.250 & 0.170 & -0.252 & 0.161 & 0.519 & -0.149 & 0.555 & -0.150 \\
\hline E64 & -0.053 & -0.274 & -0.085 & -0.290 & 0.194 & 0.003 & 0.238 & -0.038 \\
\hline S65 & -0.193 & 0.089 & -0.198 & 0.036 & 0.618 & -0.050 & 0.663 & -0.017 \\
\hline T66 & - & - & - & - & - & - & - & - \\
\hline L 67 & - & - & - & - & 0.352 & 0.216 & 0.826 & 0.447 \\
\hline H68 & - & - & - & - & - & - & - & - \\
\hline L69 & - & - & - & - & -0.208 & -0.237 & -0.024 & -0.356 \\
\hline V70 & -0.407 & -0.104 & -0.381 & -0.198 & 0.078 & -0.204 & 0.017 & -0.277 \\
\hline L71 & -0.341 & -0.265 & -0.316 & -0.205 & -0.069 & -0.045 & -0.044 & -0.110 \\
\hline $\mathrm{R} 72$ & -0.237 & -0.114 & -0.274 & -0.156 & -0.004 & -0.040 & -0.008 & -0.046 \\
\hline L73 & -0.263 & -0.110 & -0.231 & -0.089 & -0.016 & -0.037 & -0.014 & -0.036 \\
\hline R74 & -0.163 & -0.052 & -0.171 & -0.049 & -0.009 & 0.002 & -0.008 & -0.002 \\
\hline G75 & -0.146 & -0.054 & -0.148 & -0.029 & -0.008 & -0.002 & -0.007 & -0.007 \\
\hline G76 & -0.143 & -0.037 & -0.146 & -0.037 & -0.007 & -0.003 & -0.006 & 0.004 \\
\hline
\end{tabular}


Tab. 7.14: ${ }^{1} \mathrm{H},{ }^{15} \mathrm{~N}-\mathrm{PCS}$ von S57C Tb und S57C Tm $[\mathrm{ppm}]$, berechnet im RDC-Tensor. Zusätzlich sind die Abweichungen $\Delta$ von den experimentellen Werten gegeben (siehe Tabellen 7.4 und 7.5).

\begin{tabular}{|c|c|c|c|c|c|c|c|c|}
\hline \multirow[b]{2}{*}{$\mathrm{AS}$} & \multicolumn{4}{|c|}{$\mathrm{S} 57 \mathrm{C} \mathrm{Tb}$} & \multicolumn{4}{|c|}{$\mathrm{S} 57 \mathrm{C} \mathrm{Tm}$} \\
\hline & ${ }^{1} \mathrm{H}$ (ber) & $\Delta{ }^{1} \mathrm{H}$ & ${ }^{15} \mathrm{~N}$ (ber) & $\Delta{ }^{15} \mathrm{~N}$ & ${ }^{1} \mathrm{H}$ (ber) & $\Delta{ }^{1} \mathrm{H}$ & ${ }^{15} \mathrm{~N}$ (ber) & $\Delta{ }^{15} \mathrm{~N}$ \\
\hline Q2 & - & - & - & - & - & - & - & - \\
\hline I3 & - & - & - & - & - & - & - & - \\
\hline $\mathrm{F} 4$ & 0.428 & -0.298 & 0.293 & -0.323 & -0.194 & 0.215 & -0.185 & 0.257 \\
\hline V5 & 0.132 & -0.219 & 0.206 & -0.204 & -0.145 & 0.123 & -0.155 & 0.144 \\
\hline K6 & 0.210 & -0.120 & 0.171 & -0.122 & -0.117 & 0.075 & -0.109 & 0.086 \\
\hline $\mathrm{T} 7$ & 0.077 & -0.077 & 0.096 & -0.100 & -0.070 & 0.052 & -0.074 & 0.041 \\
\hline $\mathrm{L} 8$ & 0.087 & -0.043 & 0.083 & -0.043 & -0.058 & 0.033 & -0.056 & 0.036 \\
\hline $\mathrm{T} 9$ & 0.054 & -0.035 & 0.051 & -0.053 & -0.048 & 0.022 & -0.044 & 0.021 \\
\hline G10 & 0.049 & -0.036 & 0.039 & -0.034 & -0.046 & 0.027 & -0.042 & 0.010 \\
\hline K11 & 0.033 & -0.038 & 0.023 & -0.061 & -0.050 & 0.035 & -0.049 & 0.029 \\
\hline $\mathrm{T} 12$ & -0.025 & -0.085 & -0.006 & -0.100 & -0.054 & 0.039 & -0.060 & 0.035 \\
\hline $\mathrm{I} 13$ & 0.033 & -0.141 & -0.010 & -0.107 & -0.102 & 0.077 & -0.095 & 0.101 \\
\hline $\mathrm{T} 14$ & -0.204 & -0.210 & -0.177 & -0.208 & -0.112 & 0.094 & -0.123 & 0.140 \\
\hline L15 & -0.218 & -0.655 & -0.299 & -0.745 & - & - & - & - \\
\hline E16 & - & - & - & - & - & - & - & - \\
\hline V17 & - & - & - & - & - & - & - & - \\
\hline E18 & - & - & - & - & - & - & - & - \\
\hline $\mathrm{S} 20$ & - & - & - & - & - & - & - & - \\
\hline D21 & - & - & - & - & - & - & - & - \\
\hline $\mathrm{T} 22$ & - & - & - & - & - & - & - & - \\
\hline $\mathrm{I} 23$ & 0.618 & -0.137 & 0.694 & -0.118 & - & - & - & - \\
\hline N25 & - & - & - & - & - & - & - & - \\
\hline V26 & - & - & - & - & -0.215 & -0.225 & -0.236 & -0.177 \\
\hline $\mathrm{K} 27$ & 0.437 & -0.168 & 0.402 & -0.093 & -0.154 & -0.084 & -0.167 & -0.003 \\
\hline A28 & 0.264 & -0.169 & 0.224 & -0.156 & -0.128 & -0.113 & -0.127 & -0.054 \\
\hline K29 & 0.232 & -0.257 & 0.164 & -0.206 & -0.194 & 0.004 & -0.179 & 0.043 \\
\hline I30 & 0.193 & -0.215 & 0.136 & -0.225 & -0.175 & 0.070 & -0.166 & 0.074 \\
\hline Q31 & 0.112 & -0.121 & 0.087 & -0.091 & -0.117 & 0.037 & -0.110 & 0.078 \\
\hline D32 & 0.036 & -0.094 & 0.019 & -0.062 & -0.114 & 0.074 & -0.104 & 0.100 \\
\hline K33 & -0.014 & -0.105 & -0.032 & -0.070 & -0.119 & 0.112 & -0.109 & 0.122 \\
\hline E34 & 0.010 & -0.080 & -0.005 & -0.072 & -0.095 & 0.076 & -0.088 & 0.074 \\
\hline G35 & 0.016 & -0.039 & 0.016 & -0.068 & -0.076 & 0.056 & -0.069 & 0.055 \\
\hline I 36 & 0.047 & -0.054 & 0.047 & -0.049 & -0.069 & 0.035 & -0.062 & 0.019 \\
\hline D39 & 0.095 & -0.028 & 0.106 & -0.051 & -0.029 & -0.027 & -0.029 & 0.012 \\
\hline Q40 & 0.099 & -0.045 & 0.100 & -0.097 & -0.035 & -0.009 & -0.032 & 0.005 \\
\hline Q41 & 0.133 & -0.051 & 0.132 & -0.106 & -0.045 & -0.002 & -0.047 & -0.007 \\
\hline $\mathrm{R} 42$ & 0.178 & -0.059 & 0.187 & -0.129 & -0.066 & 0.012 & -0.063 & -0.013 \\
\hline L43 & 0.291 & -0.066 & 0.289 & -0.112 & -0.085 & 0.007 & -0.094 & -0.030 \\
\hline $\mathrm{I} 44$ & 0.339 & -0.063 & 0.347 & -0.051 & -0.132 & 0.033 & -0.130 & 0.052 \\
\hline
\end{tabular}


Tab. 7.14: Fortsetzung.

\begin{tabular}{|c|c|c|c|c|c|c|c|c|}
\hline \multirow[b]{2}{*}{$\mathrm{AS}$} & \multicolumn{4}{|c|}{$\mathrm{S} 57 \mathrm{C} \mathrm{Tb}$} & \multicolumn{4}{|c|}{$\mathrm{S} 57 \mathrm{C} \mathrm{Tm}$} \\
\hline & ${ }^{1} \mathrm{H}$ (ber) & $\Delta{ }^{1} \mathrm{H}$ & ${ }^{15} \mathrm{~N}$ (ber) & $\Delta{ }^{15} \mathrm{~N}$ & ${ }^{1} \mathrm{H}$ (ber) & $\Delta{ }^{1} \mathrm{H}$ & ${ }^{15} \mathrm{~N}$ (ber) & $\Delta{ }^{15} \mathrm{~N}$ \\
\hline F45 & 0.389 & -0.057 & 0.411 & -0.095 & -0.166 & 0.008 & -0.177 & -0.011 \\
\hline A 46 & 0.374 & -0.042 & 0.368 & -0.053 & -0.185 & 0.023 & -0.192 & 0.025 \\
\hline G47 & 0.270 & -0.005 & 0.259 & -0.009 & -0.133 & 0.000 & -0.133 & -0.003 \\
\hline K48 & 0.298 & 0.004 & 0.266 & -0.001 & -0.145 & -0.025 & -0.123 & -0.056 \\
\hline Q49 & 0.230 & -0.008 & 0.258 & -0.033 & -0.067 & -0.047 & -0.080 & -0.007 \\
\hline $\mathrm{L} 50$ & 0.358 & -0.037 & 0.352 & 0.018 & -0.100 & -0.008 & -0.088 & 0.014 \\
\hline $\mathrm{E} 51$ & 0.346 & -0.018 & 0.332 & -0.051 & - & - & - & - \\
\hline D52 & 0.225 & -0.031 & 0.258 & -0.107 & 0.047 & -0.041 & 0.052 & -0.067 \\
\hline $\mathrm{R} 54$ & 0.290 & -0.058 & 0.254 & -0.032 & - & - & - & - \\
\hline $\mathrm{T} 55$ & - & - & - & - & - & - & - & - \\
\hline $\mathrm{L} 56$ & 1.887 & -0.146 & 1.701 & -0.052 & - & - & - & - \\
\hline C57 & - & - & - & - & - & - & - & - \\
\hline D58 & - & - & - & - & - & - & - & - \\
\hline Y59 & 0.922 & -0.237 & 0.743 & -0.260 & -0.594 & 0.226 & -0.551 & 0.256 \\
\hline N60 & 0.821 & -0.481 & 0.868 & -0.494 & -1.327 & -0.068 & -1.287 & -0.095 \\
\hline I61 & 1.657 & -0.056 & 1.558 & -0.058 & - & - & - & - \\
\hline Q62 & 1.449 & -0.067 & 1.866 & -0.048 & - & - & - & - \\
\hline K63 & - & - & - & - & - & - & - & - \\
\hline E64 & - & - & - & - & - & - & - & - \\
\hline S65 & - & - & - & - & - & - & - & - \\
\hline T66 & 0.486 & -0.072 & 0.496 & -0.100 & -0.145 & 0.135 & -0.153 & 0.191 \\
\hline $\mathrm{L} 67$ & 0.451 & -0.137 & 0.475 & -0.126 & -0.190 & 0.129 & -0.193 & 0.142 \\
\hline H68 & 0.376 & -0.057 & 0.342 & -0.047 & -0.158 & 0.050 & -0.147 & 0.105 \\
\hline L69 & 0.202 & -0.076 & 0.225 & -0.081 & -0.101 & 0.046 & -0.106 & 0.015 \\
\hline V70 & 0.190 & -0.056 & 0.172 & -0.010 & -0.079 & 0.026 & -0.075 & 0.055 \\
\hline L71 & 0.107 & -0.047 & 0.113 & -0.057 & -0.050 & 0.026 & -0.051 & 0.014 \\
\hline $\mathrm{R} 72$ & 0.095 & -0.045 & 0.091 & -0.103 & -0.038 & 0.018 & -0.036 & 0.036 \\
\hline L73 & 0.065 & -0.049 & 0.064 & -0.076 & -0.024 & 0.012 & -0.024 & 0.031 \\
\hline R74 & 0.051 & -0.043 & 0.050 & -0.067 & -0.017 & 0.025 & -0.016 & 0.025 \\
\hline G75 & 0.041 & -0.045 & 0.041 & -0.059 & -0.012 & 0.013 & -0.011 & 0.018 \\
\hline G76 & 0.036 & -0.032 & 0.036 & -0.047 & -0.010 & 0.004 & -0.010 & 0.011 \\
\hline
\end{tabular}


Tab. 7.15: Werte der im jeweiligen PCS-Tensor berechneten RDCs und deren Differenz $\Delta$ zum experimentellen RDC (siehe Tabellen 7.11 und 7.12) der vier anisotropen Proben $[\mathrm{Hz}]$.

\begin{tabular}{|c|c|c|c|c|c|c|c|c|c|}
\hline \multirow[b]{2}{*}{$\mathrm{AS}$} & \multicolumn{2}{|c|}{$\mathrm{T} 12 \mathrm{C} \mathrm{Tb}$} & \multicolumn{2}{|c|}{$\mathrm{T} 12 \mathrm{C} \mathrm{Tm}$} & \multirow[b]{2}{*}{$\mathrm{AS}$} & \multicolumn{2}{|c|}{$\mathrm{S} 57 \mathrm{C} \mathrm{Tb}$} & \multicolumn{2}{|c|}{$\mathrm{S} 57 \mathrm{C} \mathrm{Tm}$} \\
\hline & RDC (ber) & $\Delta \mathrm{RDC}$ & RDC (ber) & $\Delta \mathrm{RDC}$ & & RDC (ber) & $\Delta \mathrm{RDC}$ & RDC (ber) & $\Delta \mathrm{RDC}$ \\
\hline Q2 & 5.29 & -11.89 & 3.80 & -5.19 & Q2 & - & - & - & - \\
\hline I3 & 7.10 & -1.88 & -9.37 & 3.10 & I3 & - & - & - & - \\
\hline $\mathrm{F} 4$ & -0.65 & 15.09 & -15.61 & 5.96 & F4 & 0.52 & -2.34 & - & - \\
\hline V5 & -3.75 & 21.56 & -14.58 & 4.65 & V5 & -0.74 & -1.00 & - & - \\
\hline K6 & - & - & -12.18 & 5.25 & K6 & -6.04 & 0.86 & -4.89 & 3.50 \\
\hline $\mathrm{T} 7$ & - & - & -0.97 & -3.85 & $\mathrm{~T} 7$ & -11.91 & 8.68 & 5.06 & -0.76 \\
\hline L8 & -3.12 & -3.14 & 7.49 & -1.99 & L8 & -5.72 & 4.70 & 7.10 & -2.32 \\
\hline T9 & 2.00 & -7.67 & 7.89 & -1.57 & Т9 & 6.94 & -4.04 & -1.83 & 0.60 \\
\hline G10 & - & - & 1.19 & 2.28 & G10 & -14.71 & 7.36 & 2.53 & -0.97 \\
\hline K11 & - & - & 3.72 & -5.70 & K11 & -5.38 & 5.61 & 8.52 & -2.85 \\
\hline $\mathrm{C} 12$ & - & - & - & - & $\mathrm{T} 12$ & -10.53 & 5.56 & 0.23 & 2.88 \\
\hline $\mathrm{I} 13$ & - & - & -12.43 & 3.16 & $\mathrm{I} 13$ & -1.15 & -0.18 & -2.62 & 2.17 \\
\hline $\mathrm{T} 14$ & -3.93 & 17.12 & -14.14 & 5.71 & $\mathrm{~T} 14$ & -2.79 & -0.33 & -6.05 & 1.00 \\
\hline L15 & 6.72 & -0.43 & -11.53 & 5.87 & L15 & 4.56 & -4.79 & - & - \\
\hline E16 & 7.33 & -8.04 & -5.34 & -0.01 & E16 & - & - & - & - \\
\hline V17 & 2.61 & -9.42 & 4.02 & -2.80 & V17 & - & - & - & - \\
\hline E18 & 4.48 & -10.52 & 4.74 & -5.95 & E18 & - & - & - & - \\
\hline $\mathrm{S} 20$ & 3.17 & 7.13 & -5.69 & -1.70 & $\mathrm{~S} 20$ & - & - & - & - \\
\hline D21 & -5.54 & -0.51 & 7.65 & -3.03 & D21 & - & - & - & - \\
\hline $\mathrm{T} 22$ & 8.03 & -2.26 & -9.41 & 6.34 & $\mathrm{~T} 22$ & - & - & - & - \\
\hline $\mathrm{I} 23$ & -12.04 & 25.41 & -9.14 & 2.08 & $\mathrm{I} 23$ & -9.21 & 3.30 & - & - \\
\hline N25 & -4.61 & 9.12 & -8.12 & 4.33 & N25 & - & - & - & - \\
\hline V26 & -8.39 & 22.74 & -11.89 & 4.52 & V26 & - & - & - & - \\
\hline $\mathrm{K} 27$ & -15.38 & 22.34 & -3.90 & 2.83 & $\mathrm{~K} 27$ & -12.31 & 6.29 & -1.82 & 1.97 \\
\hline A 28 & -9.19 & 9.61 & -2.40 & 3.55 & A28 & -7.68 & 1.91 & -5.09 & 2.49 \\
\hline K29 & -3.55 & 13.70 & -12.32 & 5.50 & K29 & -3.54 & -1.09 & -6.20 & 1.08 \\
\hline I30 & -12.05 & 22.64 & -8.99 & 4.98 & $\mathrm{I} 30$ & -9.28 & 3.11 & -3.32 & 2.57 \\
\hline Q31 & -13.18 & 14.89 & -0.42 & 3.04 & Q31 & -10.37 & 3.13 & -3.55 & 1.76 \\
\hline D32 & -5.99 & 7.54 & -4.96 & 4.41 & D32 & -5.84 & 0.35 & -5.73 & 2.12 \\
\hline K33 & -6.26 & 18.85 & -12.32 & 4.56 & K33 & -5.01 & 0.72 & -5.80 & 2.94 \\
\hline E34 & -15.11 & 19.51 & -2.23 & 3.08 & E34 & -12.05 & 5.68 & -2.40 & 2.31 \\
\hline G35 & 3.33 & -12.63 & 7.44 & -1.96 & G35 & 4.61 & -0.31 & -4.25 & 2.14 \\
\hline I36 & 6.73 & -14.67 & 4.41 & -5.68 & $\mathrm{I} 36$ & -0.26 & 2.02 & 6.13 & -2.03 \\
\hline D39 & 6.91 & -0.13 & -11.90 & 6.64 & D39 & 5.75 & -4.72 & -4.15 & 1.49 \\
\hline Q40 & 7.06 & 5.88 & -9.25 & 1.58 & $\mathrm{Q} 40$ & 11.41 & -7.01 & -4.12 & 2.03 \\
\hline Q41 & -3.96 & -4.43 & 10.11 & 0.92 & Q41 & -0.67 & 0.41 & 1.79 & -0.54 \\
\hline $\mathrm{R} 42$ & -17.29 & 14.02 & 6.20 & 1.88 & $\mathrm{R} 42$ & -13.99 & 7.67 & - & - \\
\hline L43 & -15.69 & 13.56 & 9.16 & -1.89 & L43 & - & - & - & - \\
\hline $\mathrm{I} 44$ & - & - & -4.42 & 1.72 & $\mathrm{I} 44$ & -12.55 & 6.19 & -0.35 & 1.71 \\
\hline
\end{tabular}


Tab. 7.15: Fortsetzung.

\begin{tabular}{|c|c|c|c|c|c|c|c|c|c|}
\hline \multirow[b]{2}{*}{$\mathrm{AS}$} & \multicolumn{2}{|c|}{$\mathrm{T} 12 \mathrm{C} \mathrm{Tb}$} & \multicolumn{2}{|c|}{$\mathrm{T} 12 \mathrm{C} \mathrm{Tm}$} & \multirow[b]{2}{*}{ AS } & \multicolumn{2}{|c|}{$\mathrm{S} 57 \mathrm{C} \mathrm{Tb}$} & \multicolumn{2}{|c|}{ S57C Tm } \\
\hline & RDC (ber) & $\Delta \mathrm{RDC}$ & RDC (ber) & $\Delta \mathrm{RDC}$ & & RDC (ber) & $\Delta \mathrm{RDC}$ & RDC (ber) & $\Delta \mathrm{RDC}$ \\
\hline F 45 & - & - & -3.47 & -1.46 & F45 & - & - & - & - \\
\hline A 46 & - & - & - & - & A46 & -11.31 & 5.95 & 0.83 & 0.99 \\
\hline G47 & - & - & - & - & G47 & -4.51 & -0.51 & -5.08 & 0.68 \\
\hline K48 & - & - & -0.68 & -5.35 & $\mathrm{~K} 48$ & 3.52 & -0.11 & 3.32 & 0.16 \\
\hline Q49 & -0.84 & 15.62 & -10.96 & 2.75 & Q49 & 2.52 & -0.89 & -1.94 & 1.16 \\
\hline L50 & -15.24 & 18.89 & -1.15 & 2.97 & L50 & -12.11 & 4.82 & -2.30 & 2.18 \\
\hline E51 & - & - & -3.11 & -0.15 & E51 & - & - & - & - \\
\hline D52 & 8.90 & -13.68 & 3.07 & -3.11 & D52 & 6.97 & -2.54 & 2.17 & -1.96 \\
\hline $\mathrm{R} 54$ & -3.44 & -4.61 & 7.52 & -0.89 & R54 & -0.69 & 0.73 & - & - \\
\hline $\mathrm{T} 55$ & -5.31 & 14.18 & -10.87 & 4.50 & $\mathrm{~T} 55$ & - & - & - & - \\
\hline L56 & 8.07 & 2.81 & -10.85 & 4.95 & L56 & - & - & - & - \\
\hline $\mathrm{S} 57$ & 7.10 & -4.09 & -2.81 & 2.54 & C57 & - & - & - & - \\
\hline D58 & 7.19 & -3.40 & -9.01 & 4.14 & D58 & - & - & - & - \\
\hline Y59 & 4.64 & 10.66 & -15.01 & 5.77 & Y59 & 7.05 & -3.62 & - & - \\
\hline N60 & -8.03 & -1.58 & 11.31 & -0.40 & N60 & -4.87 & 3.18 & 2.82 & 0.47 \\
\hline $\mathrm{I} 61$ & -5.31 & -3.41 & 8.66 & -0.05 & I61 & -7.64 & 5.59 & - & - \\
\hline Q62 & 5.37 & -9.78 & 5.45 & 0.45 & Q62 & 10.63 & -6.73 & - & - \\
\hline K63 & 1.59 & -12.06 & 5.35 & -0.58 & K63 & - & - & - & - \\
\hline E64 & 7.08 & -5.28 & -4.60 & 3.57 & E64 & - & - & - & - \\
\hline T66 & - & - & - & - & T66 & 5.47 & -5.28 & -5.00 & -0.41 \\
\hline $\mathrm{L} 67$ & - & - & -15.21 & 1.92 & L67 & 4.25 & -3.55 & -5.27 & 2.83 \\
\hline H68 & - & - & - & - & H68 & -12.85 & 6.27 & -0.94 & 1.08 \\
\hline L69 & - & - & 5.09 & -1.59 & L69 & -15.40 & 9.57 & 5.14 & -1.33 \\
\hline V70 & -11.18 & 3.04 & 10.99 & -1.81 & V70 & -6.82 & 2.25 & 0.40 & -0.07 \\
\hline $\mathrm{L} 71$ & - & - & 10.35 & -1.77 & L71 & -1.69 & 1.01 & 2.10 & -0.08 \\
\hline R72 & 8.40 & -5.20 & 0.82 & -1.46 & R72 & 13.59 & -8.71 & -4.18 & 1.73 \\
\hline L73 & 6.91 & -2.77 & -5.55 & 3.94 & L73 & - & - & -2.98 & 0.39 \\
\hline
\end{tabular}


Tab. 7.16: Übersicht über die Saupe-Matrixelemente der vier anisotropen Proben $\left[10^{-4}\right]$. Die gemittelten Tensoren aller Strukturen (1D3Z) sind wie folgt zugeordnet: PCS-Tensor (A), RDC-Tensor (B), Tensor aus gleichzeitiger PCS- und RDCMinimierung (C), PCSs: Metallposition bestimmt auf Basis des RDC-Tensors mit anschließender Minimierung der PCSs (D).

\begin{tabular}{|c|c|c|c|c|c|c|}
\hline Probe & Daten & $\mathrm{S}_{z z}$ & $\mathrm{~S}_{x x-y y}$ & $\mathrm{~S}_{x y}$ & $\mathrm{~S}_{x z}$ & $\mathrm{~S}_{y z}$ \\
\hline \multirow[t]{4}{*}{$\mathrm{T} 12 \mathrm{C} \mathrm{Tb}$} & $\mathbf{A}$ & 3.984 & -9.149 & -4.653 & -0.146 & -1.200 \\
\hline & B & -4.107 & -10.488 & 3.247 & -0.440 & 1.051 \\
\hline & C & -4.013 & -10.383 & 3.394 & -1.045 & 1.577 \\
\hline & D & -5.156 & -11.260 & 4.279 & -2.768 & 0.860 \\
\hline \multirow[t]{4}{*}{$\mathrm{T} 12 \mathrm{C}$ Tm } & $\mathbf{A}$ & 1.385 & 11.362 & -2.385 & -0.143 & -2.847 \\
\hline & B & -0.308 & 9.192 & -2.134 & 0.680 & -0.710 \\
\hline & C & 0.067 & 9.323 & -1.855 & 0.677 & -0.962 \\
\hline & $\mathbf{D}$ & 1.646 & 8.499 & -2.878 & -0.011 & -2.188 \\
\hline \multirow[t]{4}{*}{$\mathrm{S} 57 \mathrm{C} \mathrm{Tb}$} & $\mathbf{A}$ & 0.712 & -9.443 & -4.914 & 1.885 & -1.637 \\
\hline & B & 0.765 & -3.587 & -2.220 & 0.152 & -1.656 \\
\hline & C & 0.747 & -3.836 & -2.286 & 0.540 & -1.958 \\
\hline & D & 0.988 & -4.947 & -1.786 & 1.812 & -1.652 \\
\hline \multirow[t]{4}{*}{$\mathrm{S} 57 \mathrm{C}$ Tm } & $\mathbf{A}$ & 0.910 & 4.029 & 0.570 & -3.428 & -1.169 \\
\hline & B & 0.031 & 2.958 & 0.693 & -2.095 & -1.135 \\
\hline & C & 0.204 & 3.096 & 0.755 & -2.278 & -0.948 \\
\hline & D & 0.939 & 2.167 & -0.587 & -2.961 & -0.382 \\
\hline
\end{tabular}


Tab. 7.17: ${ }^{1} \mathrm{H},{ }^{15} \mathrm{~N}-\mathrm{PCS}$ von $\mathrm{T} 12 \mathrm{C}$ Tb und T12C Tm [ppm], zusammen mit den RDCs minimiert. Zusätzlich sind die Abweichungen $\Delta$ von den experimentellen Werten gegeben (siehe Tabellen 7.2 und 7.3).

\begin{tabular}{|c|c|c|c|c|c|c|c|c|}
\hline \multirow[b]{2}{*}{ AS } & \multicolumn{4}{|c|}{$\mathrm{T} 12 \mathrm{C} \mathrm{Tb}$} & \multicolumn{4}{|c|}{$\mathrm{T} 12 \mathrm{C} \mathrm{Tm}$} \\
\hline & ${ }^{1} \mathrm{H}$ (ber) & $\Delta{ }^{1} \mathrm{H}$ & ${ }^{15} \mathrm{~N}$ (ber) & $\Delta{ }^{15} \mathrm{~N}$ & ${ }^{1} \mathrm{H}$ (ber) & $\Delta{ }^{1} \mathrm{H}$ & ${ }^{15} \mathrm{~N}$ (ber) & $\Delta{ }^{15} \mathrm{~N}$ \\
\hline Q2 & -0.039 & -0.275 & -0.032 & -0.210 & 0.058 & 0.022 & 0.063 & 0.050 \\
\hline I3 & 0.066 & -0.159 & 0.035 & -0.192 & -0.023 & 0.067 & 0.017 & 0.101 \\
\hline $\mathrm{F} 4$ & 0.122 & 0.116 & 0.232 & 0.058 & -0.014 & 0.201 & -0.197 & 0.182 \\
\hline V5 & 0.640 & 0.239 & 0.594 & 0.341 & -0.601 & 0.024 & -0.524 & 0.038 \\
\hline K6 & - & - & - & - & -0.724 & -0.231 & -0.909 & -0.133 \\
\hline $\mathrm{T} 7$ & 1.538 & 0.298 & 1.346 & 0.204 & -0.979 & 0.065 & -0.842 & 0.056 \\
\hline L8 & 0.522 & -0.250 & 0.727 & -0.345 & -0.352 & 0.144 & -0.437 & 0.189 \\
\hline $\mathrm{T} 9$ & 0.802 & -0.198 & 0.825 & -0.234 & -0.444 & 0.168 & -0.431 & 0.187 \\
\hline G10 & 1.607 & -0.136 & 1.637 & 0.041 & -0.743 & 0.360 & -0.734 & 0.278 \\
\hline K11 & - & - & - & - & -0.810 & 0.012 & -0.926 & 0.045 \\
\hline $\mathrm{C} 12$ & - & - & - & - & - & - & - & - \\
\hline $\mathrm{I} 13$ & 0.923 & -0.070 & 0.883 & -0.399 & -0.824 & 0.170 & -0.798 & 0.350 \\
\hline $\mathrm{T} 14$ & 0.493 & -0.364 & 0.500 & -0.339 & -0.479 & 0.132 & -0.496 & 0.112 \\
\hline $\mathrm{L} 15$ & 0.229 & -0.137 & 0.229 & -0.133 & -0.211 & 0.065 & -0.208 & 0.064 \\
\hline E16 & 0.086 & -0.073 & 0.078 & -0.077 & -0.052 & 0.003 & -0.044 & 0.027 \\
\hline V17 & -0.035 & -0.054 & -0.033 & 0.005 & 0.091 & 0.012 & 0.089 & 0.007 \\
\hline E18 & -0.108 & 0.008 & -0.109 & 0.048 & 0.179 & 0.014 & 0.179 & 0.023 \\
\hline S20 & -0.159 & 0.023 & -0.174 & 0.001 & 0.230 & 0.001 & 0.250 & 0.030 \\
\hline D21 & -0.163 & 0.028 & -0.156 & 0.061 & 0.242 & 0.009 & 0.228 & 0.007 \\
\hline $\mathrm{T} 22$ & -0.134 & 0.029 & -0.154 & -0.004 & 0.190 & 0.009 & 0.218 & 0.033 \\
\hline $\mathrm{I} 23$ & -0.219 & 0.033 & -0.209 & 0.010 & 0.285 & 0.018 & 0.276 & 0.021 \\
\hline $\mathrm{N} 25$ & -0.125 & 0.025 & -0.110 & -0.012 & 0.167 & 0.011 & 0.148 & 0.048 \\
\hline V26 & -0.127 & 0.033 & -0.102 & 0.007 & 0.185 & 0.014 & 0.157 & 0.028 \\
\hline $\mathrm{K} 27$ & -0.113 & 0.031 & -0.088 & 0.028 & 0.155 & 0.007 & 0.131 & 0.006 \\
\hline A 28 & -0.058 & 0.023 & -0.041 & -0.004 & 0.087 & 0.004 & 0.068 & 0.022 \\
\hline K29 & -0.013 & 0.022 & 0.006 & -0.023 & 0.051 & 0.001 & 0.029 & 0.024 \\
\hline I30 & 0.031 & 0.023 & 0.059 & 0.009 & 0.011 & 0.003 & -0.017 & -0.002 \\
\hline Q31 & 0.035 & 0.021 & 0.056 & 0.014 & -0.009 & -0.002 & -0.029 & 0.067 \\
\hline D32 & 0.057 & 0.027 & 0.068 & -0.003 & -0.031 & -0.011 & -0.043 & 0.015 \\
\hline K33 & 0.118 & 0.025 & 0.126 & 0.018 & -0.082 & -0.011 & -0.091 & -0.004 \\
\hline E34 & 0.153 & 0.015 & 0.171 & 0.087 & -0.112 & -0.002 & -0.127 & -0.024 \\
\hline G35 & 0.111 & 0.032 & 0.114 & 0.085 & -0.083 & -0.006 & -0.088 & -0.014 \\
\hline I 36 & 0.090 & 0.020 & 0.073 & 0.042 & -0.072 & -0.001 & -0.062 & 0.009 \\
\hline D39 & -0.074 & -0.005 & -0.088 & -0.060 & 0.043 & 0.000 & 0.053 & 0.038 \\
\hline Q40 & -0.086 & -0.020 & -0.103 & -0.047 & 0.035 & 0.000 & 0.040 & 0.010 \\
\hline Q41 & -0.124 & -0.028 & -0.133 & 0.007 & 0.061 & -0.008 & 0.057 & -0.029 \\
\hline $\mathrm{R} 42$ & -0.248 & -0.030 & -0.267 & -0.003 & 0.114 & -0.060 & 0.139 & -0.034 \\
\hline L43 & -0.423 & 0.019 & -0.448 & 0.043 & 0.335 & -0.083 & 0.345 & -0.107 \\
\hline I44 & -0.842 & 0.232 & -0.921 & 0.093 & 0.745 & -0.252 & 0.798 & -0.159 \\
\hline
\end{tabular}


Tab. 7.17: Fortsetzung.

\begin{tabular}{|c|c|c|c|c|c|c|c|c|}
\hline \multirow[b]{2}{*}{$\mathrm{AS}$} & \multicolumn{4}{|c|}{$\mathrm{T} 12 \mathrm{C} \mathrm{Tb}$} & \multicolumn{4}{|c|}{$\mathrm{T} 12 \mathrm{C} \mathrm{Tm}$} \\
\hline & ${ }^{1} \mathrm{H}$ (ber) & $\Delta{ }^{1} \mathrm{H}$ & ${ }^{15} \mathrm{~N}$ (ber) & $\Delta{ }^{15} \mathrm{~N}$ & ${ }^{1} \mathrm{H}$ (ber) & $\Delta{ }^{1} \mathrm{H}$ & ${ }^{15} \mathrm{~N}$ (ber) & $\Delta{ }^{15} \mathrm{~N}$ \\
\hline F 45 & -1.595 & -0.243 & -1.734 & -0.284 & 1.600 & 0.110 & 1.864 & 0.101 \\
\hline A 46 & - & - & - & - & - & - & - & - \\
\hline G47 & - & - & - & - & - & - & - & - \\
\hline K48 & - & - & - & - & 1.617 & 0.211 & 1.220 & 0.105 \\
\hline Q49 & -0.693 & -0.170 & -0.810 & -0.228 & 0.538 & -0.055 & 0.642 & 0.000 \\
\hline L50 & -0.669 & -0.039 & -0.623 & -0.020 & 0.621 & -0.014 & 0.595 & -0.028 \\
\hline E51 & -0.380 & -0.013 & -0.367 & 0.020 & 0.419 & 0.025 & 0.389 & 0.018 \\
\hline D52 & -0.224 & 0.013 & -0.230 & 0.020 & 0.225 & -0.004 & 0.243 & -0.002 \\
\hline $\mathrm{R} 54$ & -0.227 & 0.011 & -0.215 & 0.052 & 0.272 & 0.021 & 0.263 & 0.012 \\
\hline $\mathrm{T} 55$ & -0.255 & 0.031 & -0.254 & -0.018 & 0.337 & 0.028 & 0.338 & 0.072 \\
\hline L56 & -0.272 & 0.045 & -0.310 & 0.046 & 0.394 & 0.040 & 0.446 & 0.046 \\
\hline $\mathrm{S} 57$ & -0.298 & 0.065 & -0.338 & 0.070 & 0.432 & 0.024 & 0.491 & 0.037 \\
\hline D58 & -0.329 & 0.053 & -0.350 & 0.017 & 0.458 & 0.038 & 0.487 & 0.076 \\
\hline Y59 & -0.471 & 0.076 & -0.493 & 0.047 & 0.669 & 0.077 & 0.690 & 0.098 \\
\hline N60 & -0.514 & 0.161 & -0.592 & 0.247 & 0.736 & 0.029 & 0.861 & -0.014 \\
\hline $\mathrm{I} 61$ & -0.601 & 0.209 & -0.671 & 0.326 & 0.935 & 0.043 & 1.068 & -0.009 \\
\hline Q62 & -0.763 & 0.462 & -0.642 & 0.362 & 1.345 & -0.128 & 1.092 & -0.123 \\
\hline K63 & -0.360 & 0.060 & -0.370 & 0.043 & 0.533 & -0.135 & 0.572 & -0.133 \\
\hline E64 & -0.146 & -0.367 & -0.196 & -0.401 & 0.217 & 0.026 & 0.280 & 0.004 \\
\hline S65 & - & - & - & - & 0.658 & -0.010 & 0.736 & 0.056 \\
\hline T66 & - & - & - & - & - & - & - & - \\
\hline L67 & - & - & - & - & 0.371 & 0.235 & 0.850 & 0.471 \\
\hline H68 & - & - & - & - & - & - & - & - \\
\hline L69 & - & - & - & - & -0.154 & -0.183 & 0.030 & -0.302 \\
\hline V70 & -0.298 & 0.005 & -0.263 & -0.080 & 0.118 & -0.164 & 0.066 & -0.228 \\
\hline L71 & -0.234 & -0.158 & -0.217 & -0.106 & -0.027 & -0.003 & -0.009 & -0.075 \\
\hline $\mathrm{R} 72$ & -0.172 & -0.049 & -0.201 & -0.083 & 0.016 & -0.020 & 0.015 & -0.023 \\
\hline L73 & -0.201 & -0.048 & -0.175 & -0.033 & 0.006 & -0.015 & 0.005 & -0.017 \\
\hline
\end{tabular}


Tab. 7.18: ${ }^{1} \mathrm{H},{ }^{15} \mathrm{~N}-\mathrm{PCS}$ von S57C Tb und S57C Tm [ppm], zusammen mit den RDCs minimiert. Zusätzlich sind die Abweichungen $\Delta$ von den experimentellen Werten gegeben (siehe Tabellen 7.4 und 7.5).

\begin{tabular}{|c|c|c|c|c|c|c|c|c|}
\hline \multirow[b]{2}{*}{$\mathrm{AS}$} & \multicolumn{4}{|c|}{$\mathrm{S} 57 \mathrm{C} \mathrm{Tb}$} & \multicolumn{4}{|c|}{$\mathrm{S} 57 \mathrm{C} \mathrm{Tm}$} \\
\hline & ${ }^{1} \mathrm{H}$ (ber) & $\Delta{ }^{1} \mathrm{H}$ & ${ }^{15} \mathrm{~N}$ (ber) & $\Delta^{15} \mathrm{~N}$ & ${ }^{1} \mathrm{H}$ (ber) & $\Delta{ }^{1} \mathrm{H}$ & ${ }^{15} \mathrm{~N}$ (ber) & $\Delta^{15} \mathrm{~N}$ \\
\hline $\mathrm{Q} 2$ & - & - & - & - & - & - & - & - \\
\hline I3 & - & - & - & - & - & - & - & - \\
\hline $\mathrm{F} 4$ & 0.596 & -0.130 & 0.476 & -0.140 & -0.309 & 0.100 & -0.295 & 0.147 \\
\hline $\mathrm{V} 5$ & 0.239 & -0.112 & 0.310 & -0.100 & -0.248 & 0.020 & -0.264 & 0.035 \\
\hline $\mathrm{K} 6$ & 0.268 & -0.062 & 0.229 & -0.064 & -0.182 & 0.010 & -0.170 & 0.025 \\
\hline $\mathrm{T} 7$ & 0.114 & -0.040 & 0.133 & -0.063 & -0.105 & 0.017 & -0.112 & 0.003 \\
\hline L8 & 0.113 & -0.017 & 0.108 & -0.018 & -0.083 & 0.008 & -0.080 & 0.012 \\
\hline T9 & 0.076 & -0.013 & 0.071 & -0.033 & -0.069 & 0.001 & -0.063 & 0.002 \\
\hline G10 & 0.072 & -0.013 & 0.060 & -0.013 & -0.065 & 0.008 & -0.060 & -0.008 \\
\hline K11 & 0.060 & -0.011 & 0.050 & -0.034 & -0.073 & 0.012 & -0.070 & 0.008 \\
\hline $\mathrm{T} 12$ & 0.012 & -0.048 & 0.034 & -0.060 & -0.077 & 0.016 & -0.087 & 0.008 \\
\hline $\mathrm{I} 13$ & 0.103 & -0.071 & 0.058 & -0.039 & -0.166 & 0.013 & -0.154 & 0.042 \\
\hline $\mathrm{T} 14$ & -0.110 & -0.116 & -0.066 & -0.097 & -0.194 & 0.012 & -0.212 & 0.051 \\
\hline L15 & 0.057 & -0.380 & -0.045 & -0.491 & - & - & - & - \\
\hline E16 & - & - & - & - & - & - & - & - \\
\hline $\mathrm{V} 17$ & - & - & - & - & - & - & - & - \\
\hline E18 & - & - & - & - & - & - & - & - \\
\hline $\mathrm{S} 20$ & - & - & - & - & - & - & - & - \\
\hline D21 & - & - & - & - & - & - & - & - \\
\hline $\mathrm{T} 22$ & - & - & - & - & - & - & - & - \\
\hline $\mathrm{I} 23$ & 0.648 & -0.107 & 0.726 & -0.086 & - & - & - & - \\
\hline N25 & - & - & - & - & - & - & - & - \\
\hline V26 & - & - & - & - & 0.046 & 0.036 & -0.092 & -0.033 \\
\hline $\mathrm{K} 27$ & 0.483 & -0.122 & 0.454 & -0.041 & -0.076 & -0.006 & -0.145 & 0.019 \\
\hline A 28 & 0.295 & -0.138 & 0.256 & -0.124 & -0.057 & -0.042 & -0.096 & -0.023 \\
\hline K29 & 0.286 & -0.203 & 0.211 & -0.159 & -0.258 & -0.060 & -0.257 & -0.035 \\
\hline I30 & 0.257 & -0.151 & 0.198 & -0.163 & -0.279 & -0.034 & -0.280 & -0.040 \\
\hline Q31 & 0.148 & -0.085 & 0.121 & -0.057 & -0.163 & -0.009 & -0.162 & 0.026 \\
\hline D32 & 0.062 & -0.068 & 0.043 & -0.038 & -0.176 & 0.012 & -0.162 & 0.042 \\
\hline K33 & 0.023 & -0.068 & -0.001 & -0.039 & -0.213 & 0.018 & -0.195 & 0.036 \\
\hline E34 & 0.042 & -0.048 & 0.025 & -0.042 & -0.159 & 0.012 & -0.147 & 0.015 \\
\hline G35 & 0.037 & -0.018 & 0.035 & -0.049 & -0.117 & 0.015 & -0.104 & 0.020 \\
\hline I36 & 0.069 & -0.032 & 0.066 & -0.030 & -0.098 & 0.006 & -0.084 & -0.003 \\
\hline D39 & 0.105 & -0.018 & 0.118 & -0.039 & 0.002 & 0.004 & 0.002 & 0.043 \\
\hline Q40 & 0.113 & -0.031 & 0.113 & -0.084 & -0.021 & 0.005 & -0.018 & 0.019 \\
\hline Q41 & 0.150 & -0.034 & 0.151 & -0.087 & -0.033 & 0.010 & -0.042 & -0.002 \\
\hline $\mathrm{R} 42$ & 0.203 & -0.034 & 0.211 & -0.105 & -0.077 & 0.001 & -0.069 & -0.019 \\
\hline L43 & 0.319 & -0.038 & 0.319 & -0.082 & -0.090 & 0.002 & -0.110 & -0.046 \\
\hline $\mathrm{I} 44$ & 0.374 & -0.028 & 0.379 & -0.019 & -0.180 & -0.015 & -0.173 & 0.009 \\
\hline
\end{tabular}


Tab. 7.18: Fortsetzung.

\begin{tabular}{|c|c|c|c|c|c|c|c|c|}
\hline \multirow[b]{2}{*}{$\mathrm{AS}$} & \multicolumn{4}{|c|}{$\mathrm{S} 57 \mathrm{C} \mathrm{Tb}$} & \multicolumn{4}{|c|}{$\mathrm{S} 57 \mathrm{C} \mathrm{Tm}$} \\
\hline & ${ }^{1} \mathrm{H}$ (ber) & $\Delta{ }^{1} \mathrm{H}$ & ${ }^{15} \mathrm{~N}$ (ber) & $\Delta^{15} \mathrm{~N}$ & ${ }^{1} \mathrm{H}$ (ber) & $\Delta{ }^{1} \mathrm{H}$ & ${ }^{15} \mathrm{~N}$ (ber) & $\Delta{ }^{15} \mathrm{~N}$ \\
\hline F45 & 0.416 & -0.030 & 0.441 & -0.065 & -0.209 & -0.035 & -0.229 & -0.063 \\
\hline $\mathrm{A} 46$ & 0.400 & -0.016 & 0.392 & -0.029 & -0.226 & -0.018 & -0.226 & -0.009 \\
\hline G47 & 0.291 & 0.016 & 0.278 & 0.010 & -0.158 & -0.025 & -0.153 & -0.023 \\
\hline K48 & 0.318 & 0.024 & 0.285 & 0.018 & -0.170 & -0.050 & -0.146 & -0.079 \\
\hline Q49 & 0.248 & 0.010 & 0.277 & -0.014 & -0.087 & -0.067 & -0.101 & -0.028 \\
\hline L50 & 0.384 & -0.011 & 0.377 & 0.043 & -0.112 & -0.020 & -0.095 & 0.007 \\
\hline E51 & 0.369 & 0.005 & 0.354 & -0.029 & - & - & - & - \\
\hline D52 & 0.240 & -0.016 & 0.275 & -0.090 & 0.153 & 0.065 & 0.184 & 0.065 \\
\hline R54 & 0.310 & -0.038 & 0.274 & -0.012 & - & - & - & - \\
\hline $\mathrm{T} 55$ & - & - & - & - & - & - & - & - \\
\hline L56 & 1.941 & -0.092 & 1.755 & 0.002 & - & - & - & - \\
\hline C57 & - & - & - & - & - & - & - & - \\
\hline D58 & - & - & - & - & - & - & - & - \\
\hline Y59 & 0.971 & -0.188 & 0.786 & -0.217 & -0.714 & 0.106 & -0.642 & 0.165 \\
\hline N60 & 0.869 & -0.433 & 0.906 & -0.456 & -1.222 & 0.037 & -1.177 & 0.015 \\
\hline $\mathrm{I} 61$ & 1.685 & -0.028 & 1.578 & -0.038 & - & - & - & - \\
\hline Q62 & 1.456 & -0.060 & 1.859 & -0.055 & - & - & - & - \\
\hline K63 & - & - & - & - & - & - & - & - \\
\hline E64 & - & - & - & - & - & - & - & - \\
\hline S65 & - & - & - & - & - & - & - & - \\
\hline T66 & 0.546 & -0.012 & 0.571 & -0.025 & -0.209 & 0.071 & -0.224 & 0.120 \\
\hline L67 & 0.536 & -0.052 & 0.548 & -0.053 & -0.311 & 0.008 & -0.308 & 0.027 \\
\hline H68 & 0.421 & -0.012 & 0.389 & 0.000 & -0.232 & -0.024 & -0.220 & 0.032 \\
\hline L69 & 0.242 & -0.036 & 0.264 & -0.042 & -0.149 & -0.002 & -0.155 & -0.034 \\
\hline V70 & 0.218 & -0.028 & 0.200 & 0.018 & -0.105 & 0.000 & -0.102 & 0.028 \\
\hline L71 & 0.126 & -0.028 & 0.132 & -0.038 & -0.064 & 0.012 & -0.065 & 0.000 \\
\hline R72 & 0.110 & -0.030 & 0.106 & -0.088 & -0.041 & 0.015 & -0.040 & 0.032 \\
\hline L73 & 0.075 & -0.039 & 0.075 & -0.065 & -0.026 & 0.010 & -0.025 & 0.030 \\
\hline
\end{tabular}


Tab. 7.19: Werte der berechneten RDCs [Hz], zusammen minimiert mit den PCSs, sowie deren Differenz $\Delta$ zu den experimentellen RDCs (siehe Tabellen 7.11 und 7.12) für alle vier anisotropen Proben.

\begin{tabular}{|c|c|c|c|c|c|c|c|c|c|}
\hline \multirow[b]{2}{*}{ AS } & \multicolumn{2}{|c|}{$\mathrm{T} 12 \mathrm{C} \mathrm{Tb}$} & \multicolumn{2}{|c|}{$\mathrm{T} 12 \mathrm{C} \mathrm{Tm}$} & \multirow[b]{2}{*}{ AS } & \multicolumn{2}{|c|}{$\mathrm{S} 57 \mathrm{C} \mathrm{Tb}$} & \multicolumn{2}{|c|}{ S57C Tm } \\
\hline & RDC (ber) & $\Delta \mathrm{RDC}$ & RDC (ber) & $\Delta \mathrm{RDC}$ & & RDC (ber) & $\Delta \mathrm{RDC}$ & RDC (ber) & $\Delta \mathrm{RDC}$ \\
\hline $\mathrm{Q} 2$ & -6.66 & -0.06 & 0.47 & 1.86 & $\mathrm{Q} 2$ & - & - & - & - \\
\hline $\mathrm{I} 3$ & 5.38 & 0.16 & -5.77 & 0.50 & I3 & - & - & - & - \\
\hline $\mathrm{F} 4$ & 15.17 & 0.73 & -9.97 & -0.32 & $\mathrm{~F} 4$ & -2.25 & -0.43 & - & - \\
\hline V5 & 17.34 & -0.47 & -10.19 & -0.26 & V5 & -1.82 & -0.08 & - & - \\
\hline K6 & - & - & -7.30 & -0.37 & K6 & -4.80 & 0.38 & -2.66 & -1.27 \\
\hline $\mathrm{T} 7$ & - & - & -1.89 & 2.93 & $\mathrm{~T} 7$ & -4.56 & -1.33 & 4.62 & 0.31 \\
\hline L8 & -5.80 & 0.47 & 5.75 & 0.25 & L8 & -2.56 & -1.54 & 4.62 & -0.17 \\
\hline $\mathrm{T} 9$ & -7.16 & -1.49 & 7.41 & 1.09 & T9 & 3.22 & 0.32 & -1.38 & -0.15 \\
\hline G10 & - & - & 1.87 & -1.60 & G10 & -6.93 & 0.42 & 3.01 & 1.45 \\
\hline K11 & - & - & -0.81 & 1.17 & K11 & -0.04 & -0.27 & 6.04 & 0.37 \\
\hline $\mathrm{C} 12$ & - & - & - & - & $\mathrm{T} 12$ & -5.26 & -0.29 & 1.36 & -1.75 \\
\hline $\mathrm{I} 13$ & - & - & -9.57 & -0.30 & $\mathrm{I} 13$ & -1.08 & 0.24 & -1.02 & -0.57 \\
\hline $\mathrm{T} 14$ & 13.72 & 0.53 & -8.60 & -0.17 & $\mathrm{~T} 14$ & -3.76 & -0.64 & -3.84 & 1.21 \\
\hline $\mathrm{L} 15$ & 8.31 & 2.02 & -7.15 & -1.49 & L15 & -0.38 & -0.15 & - & - \\
\hline E16 & -0.10 & 0.61 & -3.59 & 1.77 & E16 & - & - & - & - \\
\hline V17 & -5.44 & 1.37 & 1.81 & 0.59 & V17 & - & - & - & - \\
\hline E18 & -6.30 & -0.26 & 0.39 & 1.60 & E18 & - & - & - & - \\
\hline $\mathrm{S} 20$ & 9.44 & -0.86 & -6.95 & 0.44 & $\mathrm{~S} 20$ & - & - & - & - \\
\hline D21 & -4.79 & 1.26 & 4.10 & -0.52 & D21 & - & - & - & - \\
\hline $\mathrm{T} 22$ & 8.32 & 2.55 & -5.76 & -2.69 & $\mathrm{~T} 22$ & - & - & - & - \\
\hline $\mathrm{I} 23$ & 12.29 & -1.08 & -5.27 & 1.79 & $\mathrm{I} 23$ & -5.71 & 0.20 & - & - \\
\hline N25 & 4.98 & 0.47 & -3.65 & 0.14 & $\mathrm{~N} 25$ & - & - & - & - \\
\hline V26 & 13.08 & -1.27 & -6.97 & 0.41 & V26 & - & - & - & - \\
\hline $\mathrm{K} 27$ & 7.29 & 0.33 & -1.00 & 0.07 & $\mathrm{~K} 27$ & -6.85 & -0.83 & -0.11 & -0.26 \\
\hline A 28 & 0.75 & 0.33 & 0.86 & -0.29 & A28 & -5.21 & 0.56 & -2.97 & -0.37 \\
\hline K29 & 10.25 & 0.10 & -6.95 & -0.13 & K29 & -4.21 & 0.42 & -4.10 & 1.02 \\
\hline I30 & 11.83 & 1.24 & -5.02 & -1.01 & I30 & -5.83 & 0.34 & -1.30 & -0.55 \\
\hline Q31 & 1.07 & -0.64 & 2.34 & -0.29 & Q31 & -6.14 & 1.10 & -1.58 & 0.21 \\
\hline D32 & 1.89 & 0.34 & -1.21 & -0.66 & D32 & -4.64 & 0.85 & -3.67 & -0.07 \\
\hline K33 & 11.97 & -0.62 & -7.05 & 0.71 & K33 & -4.68 & -0.39 & -3.53 & -0.67 \\
\hline E34 & 4.68 & 0.28 & 0.63 & -0.22 & E34 & -6.82 & -0.46 & -0.59 & -0.50 \\
\hline G35 & -11.73 & -2.43 & 6.16 & 0.68 & G35 & 2.72 & -1.58 & -3.09 & -0.98 \\
\hline I36 & -7.01 & 0.94 & 0.00 & 1.27 & I36 & 1.91 & 0.15 & 3.62 & -0.48 \\
\hline D39 & 9.39 & 2.61 & -7.43 & -2.17 & D39 & 0.22 & -0.81 & -3.54 & -0.88 \\
\hline Q40 & 11.63 & -1.31 & -7.94 & -0.27 & Q40 & 5.50 & 1.09 & -2.72 & -0.63 \\
\hline Q41 & -8.04 & 0.35 & 9.27 & -1.76 & Q41 & -0.41 & -0.15 & 1.29 & 0.04 \\
\hline $\mathrm{R} 42$ & -1.67 & 1.60 & 6.45 & -1.63 & $\mathrm{R} 42$ & -6.68 & -0.36 & - & - \\
\hline L43 & -3.95 & -1.82 & 7.91 & 0.64 & L43 & - & - & - & - \\
\hline I 44 & - & - & -2.09 & 0.62 & I 44 & -6.55 & -0.19 & 0.99 & -0.37 \\
\hline
\end{tabular}


Tab. 7.19: Fortsetzung.

\begin{tabular}{|c|c|c|c|c|c|c|c|c|c|}
\hline \multirow[b]{2}{*}{$\mathrm{AS}$} & \multicolumn{2}{|c|}{$\mathrm{T} 12 \mathrm{C} \mathrm{Tb}$} & \multicolumn{2}{|c|}{$\mathrm{T} 12 \mathrm{C} \mathrm{Tm}$} & \multirow[b]{2}{*}{$\mathrm{AS}$} & \multicolumn{2}{|c|}{$\mathrm{S} 57 \mathrm{C} \mathrm{Tb}$} & \multicolumn{2}{|c|}{$\mathrm{S} 57 \mathrm{C} \mathrm{Tm}$} \\
\hline & RDC (ber) & $\Delta \mathrm{RDC}$ & RDC (ber) & $\Delta \mathrm{RDC}$ & & RDC (ber) & $\Delta \mathrm{RDC}$ & RDC (ber) & $\Delta \mathrm{RDC}$ \\
\hline F45 & - & - & -3.62 & 1.31 & F45 & - & - & - & - \\
\hline A 46 & - & - & - & - & A 46 & -5.50 & -0.14 & 1.80 & -0.02 \\
\hline G47 & - & - & - & - & G47 & -4.11 & 0.91 & -2.87 & 1.54 \\
\hline $\mathrm{K} 48$ & - & - & -4.17 & 1.86 & $\mathrm{~K} 48$ & 4.12 & 0.71 & 2.51 & -0.96 \\
\hline Q49 & 15.35 & 0.57 & -9.40 & -1.19 & Q49 & 1.37 & -0.27 & -0.69 & 0.09 \\
\hline L50 & 3.49 & -0.16 & 1.51 & -0.31 & L50 & -6.80 & 0.50 & -0.53 & -0.41 \\
\hline E51 & - & - & -1.64 & 1.62 & E51 & - & - & - & - \\
\hline D52 & -3.98 & 0.80 & -1.26 & -1.22 & D52 & 5.87 & 1.44 & 1.25 & 1.04 \\
\hline R54 & -10.23 & -2.18 & 7.55 & 0.92 & R54 & -0.47 & -0.51 & - & - \\
\hline T55 & 9.05 & 0.18 & -5.76 & 0.61 & $\mathrm{~T} 55$ & - & - & - & - \\
\hline L56 & 10.82 & -0.06 & -7.16 & -1.26 & L56 & - & - & - & - \\
\hline $\mathrm{S} 57$ & 2.40 & -0.61 & -0.83 & -0.56 & $\mathrm{C} 57$ & - & - & - & - \\
\hline D58 & 5.02 & 1.23 & -5.55 & -0.68 & D58 & - & - & - & - \\
\hline Y59 & 15.41 & 0.11 & -10.24 & -0.99 & Y59 & 1.31 & -2.12 & - & - \\
\hline N60 & -8.44 & 1.17 & 10.22 & -0.69 & N60 & -2.22 & -0.53 & 2.26 & -1.03 \\
\hline I61 & -6.16 & 2.56 & 6.51 & -2.10 & I61 & -3.20 & -1.15 & - & - \\
\hline Q62 & -5.08 & -0.67 & 5.16 & -0.74 & Q62 & 5.02 & 1.13 & - & - \\
\hline K63 & -10.35 & 0.12 & 5.02 & 0.25 & K63 & - & - & - & - \\
\hline E64 & 3.01 & 1.21 & -2.24 & -1.21 & E64 & - & - & - & - \\
\hline T66 & - & - & - & - & T66 & 0.00 & -0.19 & -3.99 & 1.42 \\
\hline L67 & - & - & -10.88 & 2.41 & $\mathrm{~L} 67$ & 0.52 & -0.17 & -3.32 & -0.88 \\
\hline H68 & - & - & - & - & H68 & -6.89 & -0.31 & 0.55 & 0.41 \\
\hline L69 & - & - & 4.35 & 0.85 & L69 & -6.74 & -0.91 & 4.69 & 0.88 \\
\hline V70 & -8.63 & -0.49 & 10.51 & 1.33 & V70 & -3.24 & 1.33 & 0.92 & 0.59 \\
\hline L71 & - & - & 9.45 & 0.87 & L71 & -0.86 & -0.18 & 1.56 & -0.46 \\
\hline R72 & -0.48 & -3.68 & 1.09 & 1.73 & $\mathrm{R} 72$ & 6.37 & 1.49 & -3.16 & -0.71 \\
\hline L73 & 5.54 & 1.40 & -3.11 & -1.50 & L73 & - & - & -2.53 & 0.06 \\
\hline
\end{tabular}




\section{Lebenslauf}

Name

Geburtsdatum

Geburtsort

Staatsangehörigkeit

Seit April 2007

Januar 2007 - April 2007

November 2006

Januar 2006 - November 2006

Oktober 2001 - November 2006

Oktober 2000 - August 2001

Juni 2000

September 1993 - Juni 2000

\section{Fabian Peters}

20. Januar 1981

Oldenburg

Deutsch

Doktorand in der Abteilung für NMR-basierte Strukturbiologie am Max-Planck-Institut für biophysikalische Chemie in Göttingen; Titel der Arbeit: Entwicklung von Lanthanoid-Tags für die biomolekulare NMR-Spektroskopie

Wissenschaftlicher Mitarbeiter in der Abteilung für NMR-basierte Strukturbiologie am MaxPlanck-Institut für biophysikalische Chemie in Göttingen

Diplom in Chemie

Diplomarbeit in der Abteilung für NMR-basierte Strukturbiologie am Max-Planck-Institut für biophysikalische Chemie in Göttingen; Titel der Arbeit: Synthese eines EDTA-basierten Thyminderivats, dessen Einbau in DNA und NMRspektroskopische Charakterisierung

Studium der Chemie an der Georg-AugustUniversität Göttingen, Vordiplom in Chemie im April 2004

Zivildienst im Altenzentrum Bischof Stählin in Oldenburg

Abitur

Besuch des Alten Gymnasiums zu Oldenburg 